Departamento de Mecánica de Medios Continuos

Y TEORÍA DE Estructuras

Escuela de Ingenieros de Caminos, Canales y Puertos

\title{
A STUDY OF THE MECHANICAL BEHAVIOUR OF THE HUMAN AORTIC ARTERY BY MEANS OF NON-LINEAR FINITE ELEMENT MODELS
}

SubmitTed IN PARTIAL SATISFACTION OF THE REQUIREMENTS FOR THE DEGREE

$\mathrm{OF}$

Doctor of Philosophy

\author{
A U THOR \\ CÉSAR Polindara
}

S U PER VISOR S

Professor José María Goicolea

Professor Sergio Blanco 
The composition of the text has been made using LATEX, TikZ, PGFPlots, Matlab, Python.

\section{Madrid 2015}

Author; César Polindara

Email; andrespolindara@gmail.com

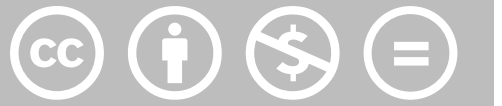


Jury appointed by the Rector of Universidad Politécnica de Madrid

President

Member

Member

Member

Secretary

Defended on ...... of February 2016 at E.T.S. de Ingenieros de Caminos, Canales y Puertos, Universidad Politécnica de Madrid

PRESIDENT

SECRETARY

$M E M B E R S$ 
In the present thesis we develop a framework for the numerical simulation of the mechanical behaviour of the human aorta using non-linear finite element models. Special attention is paid to three key aspects related to the biomechanics of soft tissues. First, the modelling of the characteristic anisotropic behaviour of the softue due to the collagen fibre families. Secondly, the modelling of damage-related softening that blood vessels exhibit when subjected to loads beyond their physiological range. And finally, the inclusion of the residual stresses in the simulations in accordance with the opening-angle experiment The modelling of damage is addressed with two major and different approaches. In the first approach a continuum local damage formulation with regularisation is presented. This formulation has two principal ingredients. On the one hand, it makes use of the principles of the smeared crack theory to avoid the mesh size dependence of the structural response in softening. On the other hand, it uses a Hodge-Petruska bidimensional model to describe the fibrils as staggered arrays of tropocollagen molecules, and from this mesoscopic model the macroscopic material properties of the collagen fibres are obtained using an homogenisation process. In the second approach a non-local gradient-enhanced damage formulation is introduced. The model is built around the enhancement of the free energy function by means of a term that contains the referential gradient of the non-local damage variable. The inclusion of this term ensures an implicit regularisation of the finite element implementation, yielding mesh-objective results of the simulations. The applicability of the later model to biomechanically-related problems is studied by means of the simulation of a typical surgical procedure, namely, the balloon angioplasty. 
En la presente tesis desarrollamos una estratégia para la simulatión numérica del comportamiento mecánico de la aorta humana usando modelos de elementos finitos no lineales. Prestamos especial atención a tres aspectos claves relacionados con la biomecánica de los tejidos blandos. Primero, el análisis del comportamiento anisótropo característico de los tejidos blandos debido a las familias de fibras de colágeno. Segundo, el análisis del ablandamiento presentado por los vasos sanguíenos cuando estos soportan cargas fuera del rango de funcionamiento fisiológico. Y finalmente, la inclusión de las tensiones residuales en las simulaciones en concordancia con el experimento de apertura de ángulo. El análisis del daño se aborda mediante dos aproximaciones diferentes. En la primera aproximación se presenta una formulación de daño local con regularización. Esta formulatión tiene dos ingredientes principales. Por una parte, usa los principios de la teoría de la fisura difusa para garantizar la objetividad de los resultados con diferentes mallas. Por otra parte, usa el modelo bidimensional de Hodge-Petruska para describir el comportamiento mesoscópico de los fibriles. Partiendo de este modelo mesoscópico, las propiedades macroscópicas de las fibras de colágeno son obtenidas a través de un proceso de homogenización. En la segunda aproximación se presenta un modelo de daño no-local enriquecido con el gradiente de la variable de daño. El modelo se construye a partir del enriquecimiento de la función de energía con un término que contiene el gradiente material de la variable de daño no-local. La inclusión de este término asegura una regularización implícita de la implementación por elementos finitos, dando lugar a resultados de las simulaciones que no dependen de la malla. La aplicabilidad de este último modelo a problemas de biomecánica se estudia por medio de una simulación the un procedimiento quirúrgico típico conocido como angioplastia de balón. 


\section{ACKNOWLEDGEMENT}

This work was completed during the period 2010-2013 at the Department for Continuum Mechanics and Theory of Structures at the Technical University of Madrid, thanks to the funding received from the Spanish Ministry of Science and Innovation through the research projects DPI-2008-03130 and DPI-2011-27609, and during the period 2013-2015 at the Institute for Mechanics at Technical University of Dortmund.

First of all I want to thank my advisors in the Technical University of Madrid, Prof. José María Goicolea and Prof. Sergio Blanco, for their continuous support and patience during the four years I spent in Madrid, and during the remainder of the time that passed until the final completion of my thesis. My special gratitud to Prof. Andreas Menzel at the Technical University of Dortmund, for giving me the opportunity to join his team as an assistant researcher at the Institute for Mechanics and to continue, at the same time, with the writing of my dissertation. To my co-author, Dr. Tobias Waffenschmidt, also my sincere gratitude for all the effort he put in the development of the non-local damage formulation, but specially for his friendship and support during my time in Dortmund. An special feeling of gratitude for Yolanda Cabrero, in Madrid, and Christina McDonagh and Kerstin Walter in Dortmund. With their support they certainly made this journey a lot easier.

Life has been definitely better with the company of great friends and colleagues, so I would like to say thank you: In Madrid, to Dr. Javier Oliva, Dr. Pablo Antolín, Dr. Khanh Nguyen, Dr. Alfredo Cámara, Dr. Mustapha El Hamdaoui, Dr. Sergio Conde, Dr. Damon Afkari, Dr. Michiel Fenaux, Dr. Roberto Ortega, Lino Dinoi, Oscar González, Ivan Rodriguez, and of course to my former flatmates, Chus and Javi. In Dortmund to "El comandante" Raphael Holtermann, Dr. Richard Ostwald, Tilman Schröder, Dr. Noy Cohen, Dr. Krishnendu Haldar, Dr. Guillermo Díaz, Dr. Thorsten Bartel, Dr. Björn Kiefer, Alexander Bartels, Dinesh Kummar, Karsten Buckmann, Rolf Berthelsen, and the rest of the members of this incredible group. Also to my flatmates, Christian, Chrissy, Julius and Isabell, they are like family and another reason to love Dortmund so much. And of course to the "Mensa Crew", Diego, Lili, Karo, "El peludo", "El original", and the rest. 
I am sure I am leaving out so many names, I apologize for that.

Finally, but most importantly, I thank my family. My father, my mother, and my dear brother. All the happiness in the world means nothing if you cannot share it with the people you love. You are a blessing.

César Andrés Polindara. 
1 Introduction $\quad 1$

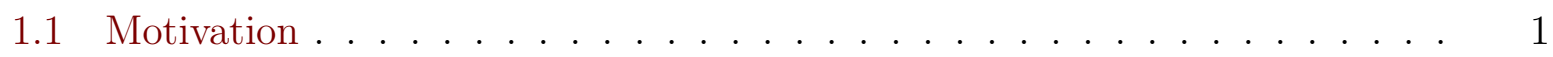

1.2 Literature review . . . . . . . . . . . . . . . . . . . 2

1.2.1 Anatomy of the cardiovascular system . . . . . . . . . . . 2

1.2.2 Histology of the arterial wall . . . . . . . . . . . . . . . 4

1.2.3 Mechanical behaviour of the arterial wall . . . . . . . . . . . . 5

1.2.4 Modelling of damage in fibrous soft tissues . . . . . . . . . . . 8

1.3 Objectives . . . . . . . . . . . . . . . . . . . . . . . 10

1.4 Scope . . . . . . . . . . . . . . . . . . . . . . 10

2 Hyperelastic constitutive models for soft biological tissues $\quad 13$

2.1 Basic kinematics . . . . . . . . . . . . . . . . . . . 13

2.2 Hyperelasticity . . . . . . . . . . . . . . . . . . . . . . . . . . . . . . . . 15

2.2.1 Hyperelasticity with no internal dissipation . . . . . . . . . . . 15

2.2.2 Hyperelasticity with damage . . . . . . . . . . . . . . 16

2.3 Constitutive modelling of a soft fibre-reinforced biological tissue . . . . . . 17

3 Regularised local continuum damage model based on the mesoscopic scale $\begin{array}{ll}\text { for fibred biological tissues } & 19\end{array}$

3.1 Local damage model with regularised softening . . . . . . . . . . . . . . . . . 19

3.1 .1 Ingredients of the local damage model . . . . . . . . . . . . . 20

3.1.2 Internal dissipation and softening regularisation . . . . . . . . . . 22

3.1.3 Summary of material parameters . . . . . . . . . . . . . 23

3.2 Mesoscopic characterisation of fibre inelastic behaviour . . . . . . . . . . 24

3.2.1 Fibril mesoscopic model . . . . . . . . . . . . . . . 25

3.2.2 Fibre macromechanical characterisation . . . . . . . . . . . . 31

3.3 Numerical examples . . . . . . . . . . . . . . . . . . . . . . . . . . . 35

3.3.1 Fibril behaviour analysis . . . . . . . . . . . . . . 35

3.3.2 Fibre behaviour analysis . . . . . . . . . . . . . . . 36

3.3 .3 Soft tissue behaviour analysis . . . . . . . . . . . . . . . . 37

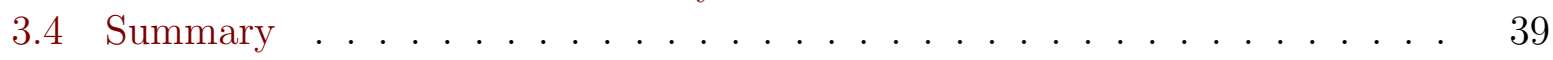

4 Non-local gradient-enhanced continuum damage model for fibred biological tissues

4.1 Gradient enhancement of a continuum damage formulation . . . . . . . . . 44

4.1.1 Total potential energy and variational formulation . . . . . . . . . . 44

4.1.2 Strong form - Euler-Lagrange equations . . . . . . . . . . . . 46 
4.2 Constitutive relations . . . . . . . . . . . . . . . . . . . . 47

4.2.1 Gradient-enhanced part of the free energy function . . . . . . . . . 47

4.2.2 Gradient-enhanced damage model - continuous formulation . . . . 48

4.2.3 Gradient-enhanced damage model - algorithmic setting . . . . . . . 49

4.3 Finite element discretisation . . . . . . . . . . . . . . . . . 54

4.3.1 Discretisation . . . . . . . . . . . . . . . 55

4.3 .2 Linearisation . . . . . . . . . . . . . . . . 57

4.3.3 Assembly of the global system of equations . . . . . . . . . . . 58

4.3.4 Q1P0 finite element formulation . . . . . . . . . . . . . 59

4.4 Numerical examples . . . . . . . . . . . . . . . . . . . . . . . . . 60

4.4.1 Test 1 - Homogeneous uniaxial tension . . . . . . . . . . . . 62

4.4.2 Test 2 - Plate with a hole . . . . . . . . . . . . . . . 64

4.5 Summary . . . . . . . . . . . . . . . . . . . . 74

$\begin{array}{lll}5 & \text { Clinical applications } & 77\end{array}$

5.1 Residual stresses in arteries . . . . . . . . . . . . . . . . 77

5.2 Finite element modelling of damage in balloon angioplasty . . . . . . . . 79

5.2.1 Setup of the boundary value problem . . . . . . . . . . . 83

5.2 .2 Results. . . . . . . . . . . . . . . . . 86

5.3 Finite element modelling of a full-3d artery-like tube . . . . . . . . . . . . . 98

5.3.1 Setup of the boundary value problem . . . . . . . . . . . 98

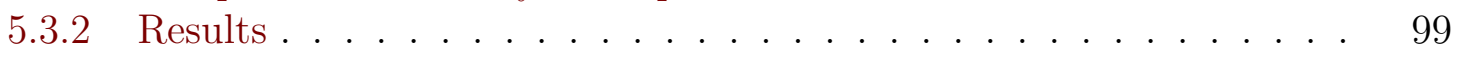

5.4 Summary . . . . . . . . . . . . . . . . . . . . . 103

6 Conclusion $\quad 107$

6.1 Synthesis and conclusions . . . . . . . . . . . . . . . . 107

6.1.1 Continuum local damage formulation with regularisation . . . . . . 107

6.1.2 Non-local gradient-enhanced continuum damage formulation . . . . 108

6.2 Main contributions . . . . . . . . . . . . . . . . . . 109

6.3 limitations and future perspectives . . . . . . . . . . . . . . . . 109

6.4 Publications . . . . . . . . . . . . . . . . . . . . . 110

$\begin{array}{ll}\text { A Local tangent moduli } & 113\end{array}$

$\begin{array}{lr}\text { Bibliography } & 116\end{array}$ 

Content 


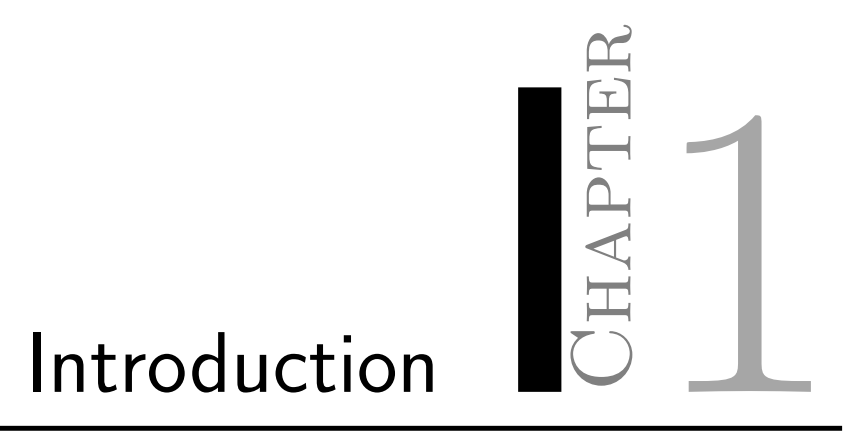

\subsection{Motivation}

Cardiovascular Diseases (CVDs) are a group of pathologies of the Cardiovascular System that affect primarily the heart and the blood vessels, and accordingly to World Health Organization [1], one of the leading causes of mortality amongst the world population. The aforementioned study reveals that solely in 2012 an estimated of 17.5 million people died because of related CVDs, a number that represents a $31 \%$ of all deaths worldwide in that year. Most cardiovascular diseases can be prevented by addressing behavioural risk factors such as tobacco use, unhealthy diet and obesity, physical inactivity and harmful use of alcohol. Nonetheless, people with cardiovascular disease or who are at high cardiovascular risk (when presenting one or more risk factors such as smoking, high cholesterol levels, hypertension or diabetes, to name only a few) require prompt medical evaluation and treatment with medication, and in severe cases of acquired pathologies like atherosclerosis, aortic aneurysm or aortic dissection, might also require surgical intervention. Typical surgical procedures for common CVDs are coronary artery bypass, balloon angioplasty (where a small balloon-like device is threaded through an artery to open the blockage), valve repair and replacement, and in extreme cases of heart failure heart transplantation. Balloon angioplasty, specifically, stands out from the rest of the aforementioned procedures for being a minimally invasive procedure. Balloon angioplasty is a common surgical intervention used to extend or reopen narrowed blood vessels in order to restore the continuous blood flow in, for instance, atherosclerotic arteries. A balloon, typically made of a highly flexible rubber-like plastic sheet, is inserted within the blocked artery and inflated hence reopening up the blocked blood vessel for improved blood flow. A stent may or may not be inserted at the time of the intervention to ensure the vessel remains open. Balloon angioplasty is a widely used treatment for atherosclerosis in carotid arteries and the aorta, however, this procedure carries the risk of restenosis and arterial wall complications. The 
technique of balloon angioplasty involves expansion of the blood vessel which might result in rupture of the intima and injury of the media (Lock et al. [2]). Furthermore, post procedural complications after balloon angioplasty include injury at the percutaneous access site, elastic recoil or restenosis, and uncontrolled damage of the aortic wall resulting in aortic aneurysm formation (Beekman et al. [3]; Rao and Carey [4]; Rao [5]). Even though the common agreement that acute mechanisms of balloon angioplasty are largely mechanical, there still few detailed mechanical studies devoted to quantify balloon-induced damage to the arterial wall. The present dissertation aims to develop a finite element framework that serves to describe the mechanical behaviour of the arterial wall.

\subsection{Literature review}

In this section we present the literature review. In section 1.2.1, we discuss basic anatomical concepts of the cardiovascular system. In section 1.2.2, a detailed description of the composition and histology of healthy blood vessels is presented. In section 1.2.3, biomechanical concepts related to the characteristic mechanical behaviour of blood vessels is presented. Finally, in section 1.2.4, provided clinical application of blood vessels simulations, like balloon angioplasty, are strongly related to inelastic phenomena such as damage, we make a review on the modelling of damage in soft tissues.

\subsubsection{Anatomy of the cardiovascular system}

The human cardiovascular system consists of a collection of organs that allow blood to transport substances, such as nutrients and waste products, to and out of the cells. The essential organs that form the cardiovascular system are the heart and the blood vessels, in addition to the blood, which is a tissue by itself. The cardiovascular system comprises two primary circulation loops: Pulmonary circulation and the systemic circulation. The first loop transports deoxygenated blood from the right side of the heart to the lungs, where the blood picks up oxygen, and then back to the left side of the heart. On the other hand, the second loop transports highly oxygenated blood from the left side of the heart to all of the tissues of the body (with the exception of the heart and lungs) and then back again to the right side of the heart. Blood circulation is driven by the continuous and periodic motion of the heart, where two stages are identified: diastole and systole. The first stage corresponds to a period where the heart is relaxing and filling itself with blood, and the latter corresponds to a period where the heart is contracting itself and pumping blood into the circulation loops. Furthermore, as blood flows through the circulatory system a pressure load is exerted upon the wall of the blood vessels. This mechanical load is called blood pressure and it is measured in milimeters of mercury [ $\mathrm{mmHg}$ ], with 


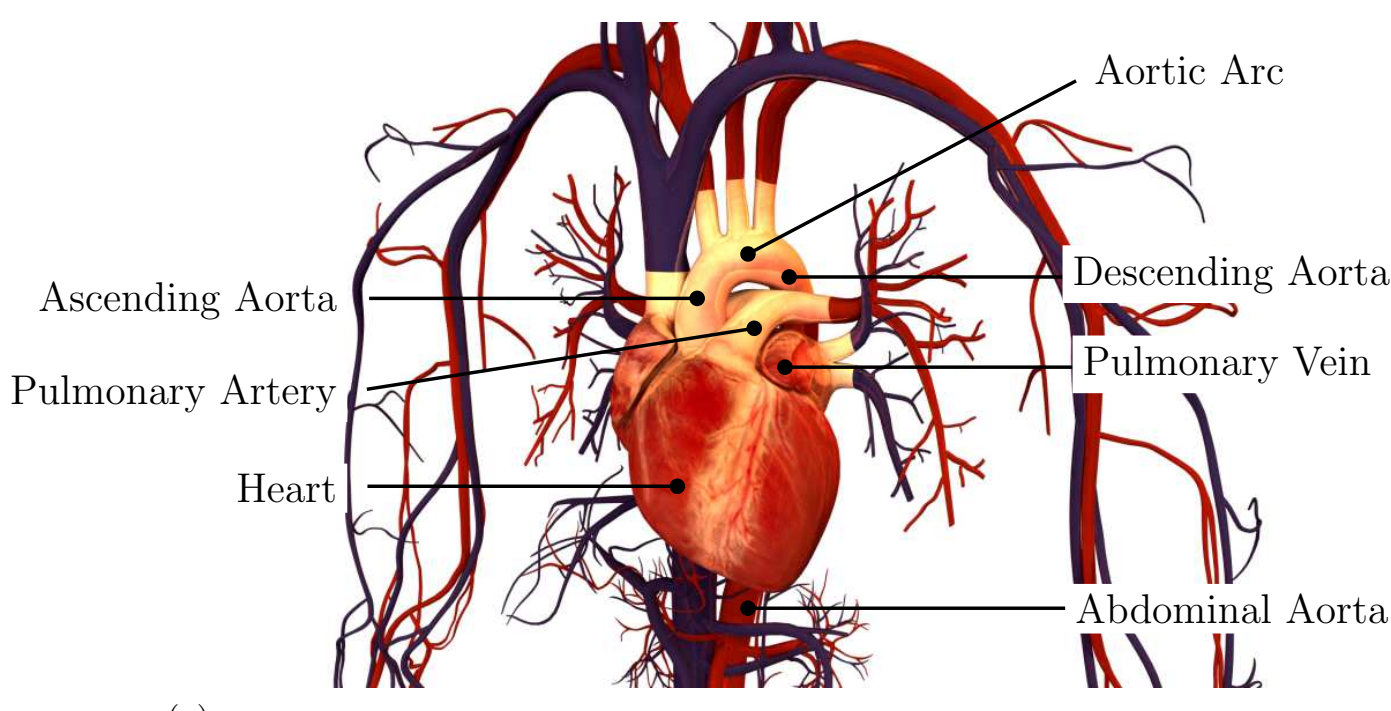

(a)

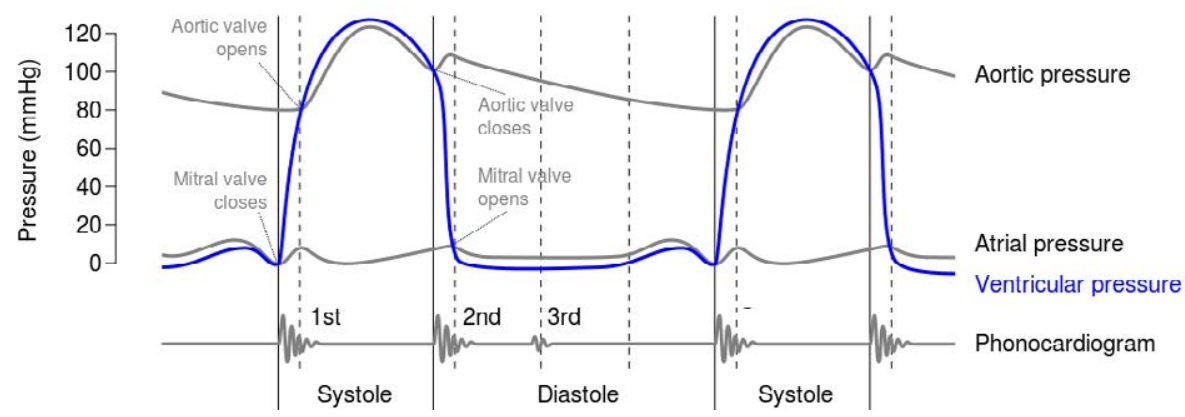

(b)

Figure 1.1: (a)Schematic of the human cardiovascular system in the upper part of the body (European Journal of Cardiovascular Prevention and Rehabilitation, 2014). Main sections of the aorta artery are highlighted. (b)Wiggers diagram. Blood pressure history in humans (Wikimedia Commons).

$100[\mathrm{mmHg}]=13.33[\mathrm{kPa}]$. The largest variation in blood pressure occurs in the left ventricle of the heart, where its lowest value lies between 3 and $12[\mathrm{mmHg}]$ during diastole, and its highest value lies between 100 and 140 [mmHg] during systole Klingensmith et al. [8]. An schematic of blood pressure variation with time for an adult healthy person is presented in Figure 1.1b. There it can be appreciated that blood pressure in the left ventricle has a wide range of variation, from 0 up to a little bit over $120[\mathrm{mmHg}]$. Blood pressure in the aorta, on the other hand, varies between 80 (diastole) and 120 (systole) [mmHg]. Moreover, during systole, pressure in the left ventricle is larger than in the aorta, since blood flow occurs in the direction of the aortic artery. Finally, there are two types of blood vessels: arteries and veins. Although they share a common distinctive function, namely blood transport, there are several major differences between them, from which we name only the following

- Direction of the blood flow: Arteries transport blood away from the heart while veins transport blood to the heart. 


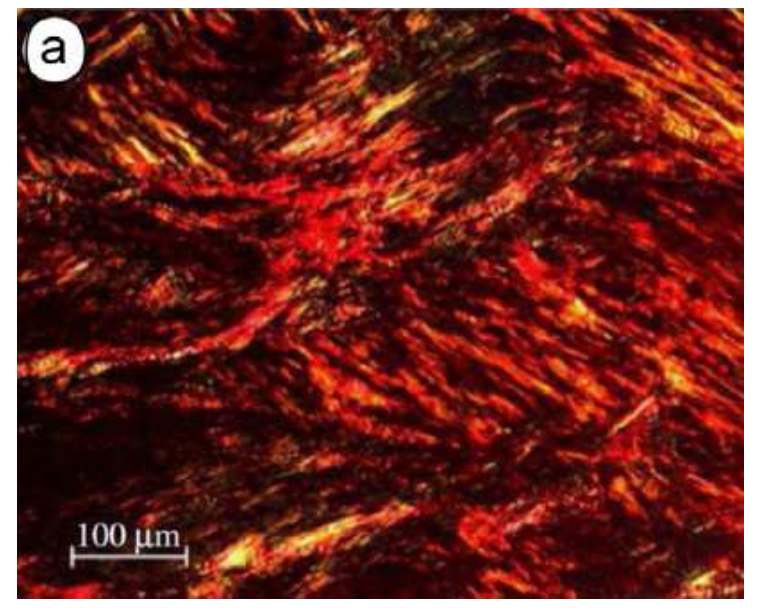

(a)

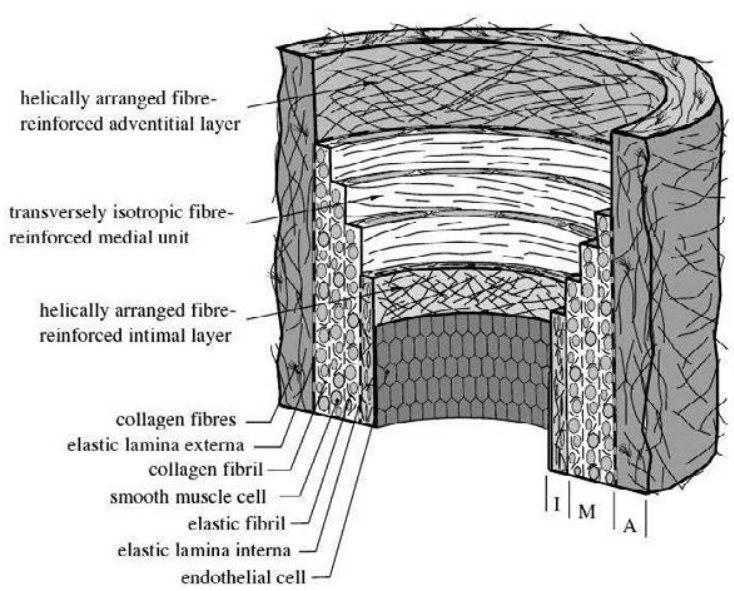

(b)

Figure 1.2: (a) Polarised light micrograph of the media. The image show the helical orientation of the two collagen fibre families. Reprinted from Schriefl et al. [6], with kind permission from Royal Society Publishing. (b) Multilayered structure of the arterial wall. Reprinted from Gasser et al. [7], with kind permission from Royal Society Publishing.

- Blood oxygen content: Arteries transport oxygenated blood while veins transport deoxygenated blood, with the only exception being the pulmonary artery and the pulmonary vein, which transport deoxygenated and oxygenated blood, respectively.

- Blood pressure: In arteries the blood pressure is consistently higher than in veins.

- Blood volume: Blood content in arteries is lower than in veins. Blood content in arteries is $15 \%$ of the total blood content in the human body, while in veins is $65 \%$. The remainder $20 \%$ is divided amongst the heart, the lungs and the capillaries.

- Compliance: Veins have a larger compliance than arteries.

\subsubsection{Histology of the arterial wall}

Here we discuss the histology of the arterial wall. Three different layers can be differentiated in the arterial wall: the intima, the media and the adventitia (see Figure 1.2(b)).

Intima: It is the interior layer of the arterial wall, and it is formed by a layer of endothelial cells and a layer of subendothelial cells. In healthy arteries the intima does not contribute to the mechanical response of the tissue. Nonetheless, when pathologies like the atherosclerosis affect the tissue, the intima increases its thickness and stiffness due to the accumulation of substances like fat, calcium, collagen fibres and fibrin. These pathological changes are associated with important alterations in the geometry and in the mechanical properties of the tissue, to the point that the contribution of the intima to the artery's mechanical response might become considerable. 
A first studio on the issue of calcification of the intima and the mechanical implication of atheromatous plaques in the arterial wall is found in Duguid and Robertson [9]. Further details about the physicochemical, epidemiological and clinical aspects of atherosclerosis can be found in Schmid et al. [10]; Wexler et al. [11].

- Media: It is the intermedial layer of the arterial wall, and it is mainly formed by muscular cells, elastin and collagen fibres. The collagen fibres are grouped into two fibre families which are helically oriented along the axial axis of the artery. It is this particular disposition of the collagen fibres which allows the artery to support large loads in the circumferential direction.

- Adventitia: It is the external layer of the arterial wall, and it is mainly composed of fibroblasts and fibrocytes, a base histological substance (hereafter known as ground substrate or ground matrix) and collagen fibres. As it is for the media, these collagen fibres are also grouped into two fibre families which are helically oriented along the axial axis of the artery.

The main components forming the different layers of the arterial wall are the following:

- Elastin: It is a fibrous protein, that from the mechanical point of view presents a pronounced linear stress-stretch response Fung [12]. It maintains its elastic properties up to stretch values of $\lambda=l / L=1.6$, being $l$ and $L$ the undeformed and deformed longitude of a given elastin fibre, respectively.

- Collagen: It is a basic structural element in both, hard and soft tissues. It is a fibrous protein that, along with the elastin, form most of the bulk of the extracellular matrix in soft tissues. Collagen is found in a crimped shape (see Figure 1.2(a)), and it is stretched and reoriented under the action of mechanical loads. For this reason the contribution of collagen fibres to the mechanical response of soft tissues is only appreciable at large states of deformation.

\subsubsection{Mechanical behaviour of the arterial wall}

In the following we describe some of the characteristic features of the mechanical response of the arterial wall. In particular we address the following: anisotropy; inelastic behaviour, namely viscoelasticity and damage; mechanobiological processes, namely growth and remodeling, and finally residual stresses. The description herein presented relies in its majority on the seminal textbook by Fung [12] as well as on the more recent monograph by Humphrey [13] which provides a comprehensive review of the biology and the biomechanics of the human cardiovascular system. 


\section{Stiffening and anisotropy}

Healthy arteries are able to withstand large deformations and exhibit a highly non-linear stress-stretch response, in addition to a large stiffening that is appreciable within the physiological load range. The stiffening is explained by the reorientation of the collagen fibres when the tissue is loaded. In a load-free state the collagen fibres are crimped (see Figure 1.2(a) and do not contribute to the mechanical response of the artery. Nonetheless, when the tissue is progressively loaded, the collage fibres are stretched and reoriented at the same time, hence increasing the load-bearing capacity of the tissue. This process where the collagen is simultaneously stretched and reoriented is called fibre recruitment, and it is more accentuated in the adventitia. This characteristic behaviour of the tissue reveals the important role of collagen fibres, since the constitute the main load-bearing components in blood vessels. Furthermore, the collagen is in great part responsible for the characteristic anisotropic response of the artery. The helical orientation of the the fibre families along the artery's axial direction, confers to the material a cylindrical orthotropic response.

\section{Viscoelasticity and damage}

The human body is mainly composed of water, a situation that partly explains why biological tissues behave under specific circumstances as solid or fluids. Experimental findings reveal that the cardiovascular tissue exhibits characteristic viscoelastic features, such as creep, relaxation and hysteresis. This viscoelastic behaviour is more pronounced in distal arteries (arteries far from the heart) than in proximal arteries (arteries close to the heart). In addition to the viscoelastic response, another dissipative phenomena can be observed when blood vessels are loaded beyond their physiological load range, as it is the case for surgical procedures as the balloon angioplasty. This dissipative or inelastic phenomena also known as damage, triggers a progressive degradation in the load-bearing capacity of the tissue, a phenomena called softening. Figure 1.3(a) shows the characteristic response of a fascia tissue when uniaxially loaded beyond its physiological load-range. After the peak load is reached, a dramatic reduction in the load-bearing capacity of the tissue, i.e. softening, becomes apparent. Figure 1.3(b) shows the same characteristic response in an artery which is inflated beyond the physiological blood-pressure range.

\section{Growth, remodeling and residual stresses}

It was a long standing assumption, that the most relevant characteristic features of the mechanical response of soft tissues was their non-lineal stress-stretch response, their quasiincompressibility, their anisotropy and their inelastic response under particular situations. Nonetheless, more recent studies reveal another important characteristic of soft tissues: their ability to change its mass, growth, and their ability to change their internal structure, 


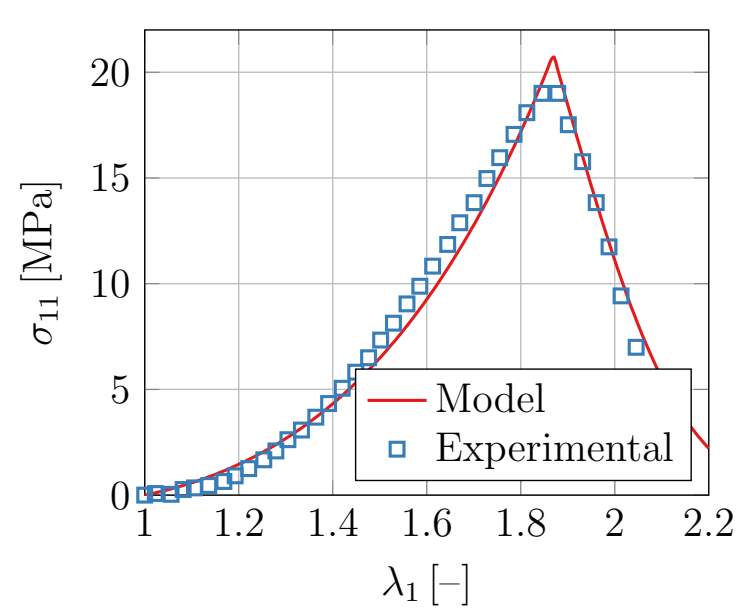

(a)

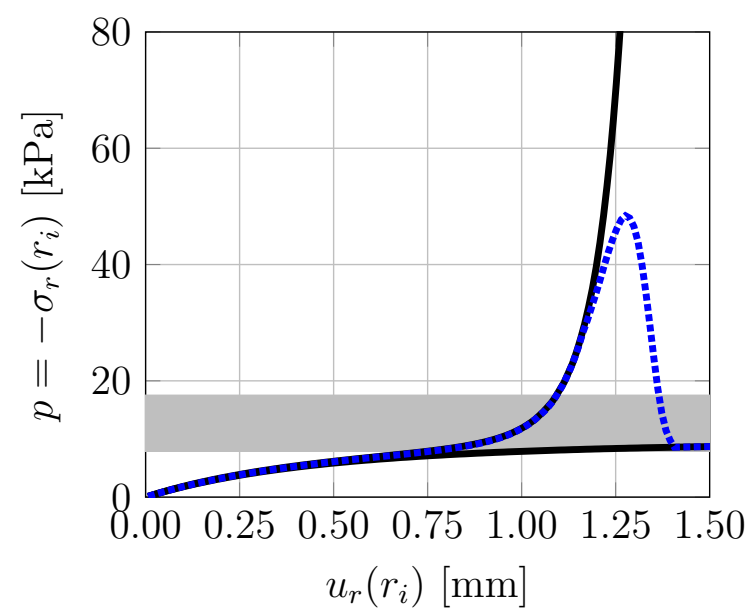

(b)

Figure 1.3: Damage and softening in soft tissues. (a) Qualitative simulation based on the damage model proposed in Chapter 4 and experimental stress-stretch data of a fascia tissue specimen under uniaxial isochoric tension, cf. Martins et al. [14]. Material parameters are chosen as $\mu_{\mathrm{e}}=0.01[\mathrm{kPa}], k_{\mathrm{e}}=499.0[\mathrm{kPa}], k_{1}=0.55[\mathrm{kPa}], k_{2}=0.01, k_{\mathrm{d}}=3.5[\mathrm{kPa}], \eta_{\mathrm{d}}=0.6\left[\mathrm{kPa}^{-1}\right]$. (b) Load-displacement response of an artery-like tube subjected to internal pressure based on the damage model proposed in Chapter 4: pressure $p$ versus radial displacement $u_{r}\left(r_{i}\right)$ at the inner radius of the artery-like tube. The lower and upper solid black curves represent the purely elastic response of the neo-Hookean ground substance and the fibre-reinforced material, respectively. The blue curve shows the mechanical response captured by the gradient-enhanced damage model proposed in Chapter 4. The gray-shaded region represents the physiological range for blood pressure.

remodelling, in response to a disease, an injury or even to subtle mechanical changes in their environment.

Growth and remodelling are irreversible processes that originate residual stresses. This hypothesis has been corroborated in several occasions. To name a few examples, Fuchs [15] noded that the in situ longitude of an artery it was longer than its corresponding ex situ value, meaning that residual stresses in the axial direction were present in the blood vessels. Further experiments also revealed that when a radial cut is performed on an artery, it springs open, meaning that also residual stresses in the circumferential direction are present. Finally, Chuong and Fung [16] pointed out that residual stresses play an important role in the mechanical behaviour of arteries. In this worked the authors note that residual stresses have a twofold beneficial effect on the blood vessels. First, the gradient of circumferential stresses along the thickness of the arterial wall is reduced, hence every cell would withstand the same load. Secondly, the residual stresses reduce the overall circumferential stress levels that the artery would have if it was not residually stressed. From a mechanical point of view these observations reveal that the residual stresses optimise the structural response of the artery. 


\subsubsection{Modelling of damage in fibrous soft tissues}

Damage processes in anisotropic soft biological tissues are closely related to the progressive failure of fibres embedded in the ambient bulk or matrix material. Arteries, for instance, can be considered as a composite of an isotropic ground substance of elastin fibres and a highly anisotropic network of cross-linked collagen fibrils. Mechanical loading beyond the physiological loading range, e.g. caused by a surgical intervention such as balloon angioplasty, can significantly reduce the elastic properties of the artery. Physically related to Mullins-type effects, these phenomena can be attributed to the continuous degradation of particular collagen fibres and to the corresponding overstretch of neighbouring cross-links leading to pronounced softening.

The structural mechanisms that control the degradation of soft tissues are related with the behaviour of their fundamental constituents. The study of the relationship between the molecular and intermolecular properties and the tissue behaviour is been actively addressed at present ([17], [18], [19], [20], [21] among others). As a consequence, several damage models for soft tissues have been presented derived from the molecular features associated with the tropocollagen molecules. A stochastic, structurally based damage model that considers statistical aspects related to the length distribution of the reinforcing fibres was presented in [22]. A reactive mesoscopic model was presented in [18], where tropocollagen molecules are described as a collection of particles interacting according to multibody potentials (see also [23]). Based on these molecular simulations, a multiscale, plasticity based, constitutive model was presented in [24]. Another plasticity based model has been presented in [25], where viscoplastic sliding of collagen fibrils is associated with the irreversible degradation of the proteoglycan bridges between them. This model has been applied to the study of the properties of the infrarenal aorta in [26] and has been enriched with a collagen turnover model in [27].

Another approach to study damage follows the classical work by Kachanov [28] who interpreted the damage effect as a consequence of an area reduction of the stress-bearing region, hence material degradation can be modelled by means of standard continuum damage formulations, i.e. in a local sense. Up to now, a large variety of models exist where we refer the reader to classic monographs and textbooks as, e.g. Kachanov [28], Kachanov [29], Krajcinovic and Lemaitre [30], Lemaitre and Chaboche [31], Lemaitre [32] or Krajcinovic [33], to name only a few. However, the assumption of a purely local continuum damage may, as a major drawback, imply a loss of well-posedness, such as loss of ellipticity, of the underlying boundary value problem. With regard to related numerical methods such as the finite element method, this results in a significant mesh-dependence of the solutions, in other words in a vanishing localised damage zone upon mesh refinement, and hence physically meaningless - or at least questionable - simulations. 
In order to regularise the problem, and thereby to circumvent the aforementioned deficiencies, several approaches have been proposed in the literature as, for instance, viscous regularisation, or the concept of generalised non-local continua such as micromorphic continua, see the monograph on non-local continuum field theories by Eringen [34], the articles by Aifantis [35, 36] or the contributions in Eringen [37] and Rogula [38]. Here, intrinsic or rather internal length scales are introduced into the continuum formulation. A non-local continuum formulation can generally be established by either introducing an integral- or a gradient-type equation.

Non-local integral models are inherently associated with a global averaging procedure which complicates the linearisation of the equations and from the computational point of view, are far more expensive than related gradient-type models. With regard to continuum damage formulations, non-local integral models are advocated by Pijaudier-Cabot and Bažant [39] and Bažant [40] and extensively studied by Jirásek [41] or Bažant and Ožbolt [42] and Bažant and Di Luzio [43], the latter referring to microplane models.

As a convenient alternative, Lasry and Belytschko [44], Mühlhaus and Alfantis [45] and Polizzotto et al. [46] suggested non-local gradient models. Here, the non-locality is incorporated by means of an additional gradient-based Euler-Lagrange equation, to be fulfilled in a weak sense, accompanied by a non-local quantity taking the interpretation of an additional independent variable. Non-local gradient-based continuum damage formulations were first discussed in de Vree et al. [47], Peerlings et al. [48] and de Borst and Pamin [49]; see also the comparison by Pamin [50] or the coupled damage-plasticity approach by Svedberg and Runesson [51].

A large variety of gradient-extended damage formulations exists for the geometrical linear case, see e.g. the contributions by Kuhl and Ramm [52], Kuhl et al. [53] for anisotropic gradient damage, the article by Liebe et al. [54] or the work by Dimitrijević and Hackl [55] for isotropic gradient damage. However, there is a comparatively small number of contributions for the geometrical non-linear case. The article by Steinmann [56] can be considered as a starting point wherein the non-local strain energy density is introduced as an additional primary variable. This approach was used in Liebe and Steinmann [57], and similarly with application to softening-plasticity in Liebe et al. [58], and compared to an alternative model which takes the damage field as an independent variable. Both approaches used a global active-set-search to account for the Kuhn-Tucker conditions. Following the concept by Frémond and Nedjar [59], the article by Nedjar [60] considered a damage-related formalism based on the principle of virtual power where the power of the internal forces depends, in addition to the strain rates, on the damage rate itself as well as on the gradient of the damage rate. The series of papers by Abu Al-Rub and Voyiadjis [61] and Voyiadjis and Abu Al-Rub [62], proposed a non-local gradient-enhanced plastic-damage model coupled to visco-inelasticity where 'explicit and implicit microstructural length scales' are 
introduced by means of viscosity and gradient localisation limiters.

\subsection{Objectives}

If the main goal of the present thesis should be summarised in one sentence, that could be expressed as

To develop a comprehensive framework for the numerical simulation of the mechanical behaviour of the human aorta using non-linear finite element models.

The modelling of the mechanical behaviour of such a complex material, like is the arterial wall, entails, of course, the necessity for the careful consideration of several aspects that define the true nature of a biological tissue. In the present thesis we address the following specific objectives:

- Use a constitutive formulation that is able to represent the anisotropic nature of the arterial wall. In particular we should use a constitutive formulation that specifically considers typical orientation of the collagen fibre families in the tissue.

- Use a constitutive formulation that is consistent with the quasi-incompressibility condition, i.e. that it is able to represent a quasi-incompressible behaviour of the arterial wall.

- Develop a procedure that allows for the consideration of the residual stresses present in the blood vessels. The proposed framework should be consistent with the experimental data related to the opening-angle experiment.

- Develop a continuum damage model formulation that is able to reproduce the characteristic softening exhibited by blood vessels when they withstand loads beyond their physiological range.

\subsection{Scope}

In the present chapter we make a literature review. In chapter 2 we present the basic concepts of continuum mechanics that define the framework of the constitutive modelling for hyperelastic materials, paying special attention to the use of structural tensors for the modelling of anisotropy. The equations herein derived are used in later developments. In chapter 3 we present a phenomenological continuous damage model with regularised softening for soft tissues. Here we consider the hierarchical structure of the collagen, which 
is made of a complex framework of fibres, fibrils, tropocollagen molecules and aminoacids. A Hodge-Petruska two-dimensional model is used to describe the fibrils as staggered arrays of tropocollagen molecules. In doing so, meso-structurally based definitions for the material parameters of the artery are obtained. In chapter 4 we present a non-local gradientenhanced continuum damage formulation at large deformations. The model is built around the enhancement of the local free energy function by means of a term that contains the referential gradient of the non-local damage variable $\phi^{i}$. The inclusion of this term ensures an implicit regularisation of the finite element implementation, ensuring mesh-objective results of the simulations. In chapter 5 we explore two boundary value problems related to biomechanics of the cardiovascular system namely the balloon angioplasty and the inflation of a full 3D artery-like tube. Specifically we here study the effect that damage evolution has on the structural response of the artery using the finite element implementation of the gradient-enhanced damage model presented in chapter 4. Special attention is devoted to the enforcement of residual stresses. Conclusions and future works are summarised in chapter 6 . 


\section{Hyperelastic constitutive models for soft biological tissues}

In this chapter we briefly present the basic concepts of continuum mechanics that define the framework of the constitutive modelling for hyperelastic materials. Special attention to the use of structural tensors for the modelling of anisotropy is given. The equations herein derived will be used in later developments. This is by no means an intensive review on solid mechanics and many important topics are omitted.

\subsection{Basic kinematics}

Let $\boldsymbol{x}=\boldsymbol{\varphi}(\boldsymbol{X}, t)$ describe the motion of the body, which transforms referential placements $\boldsymbol{X} \in \mathcal{B}_{0}$ to their spatial counterparts $\boldsymbol{x} \in \mathcal{B}_{t}$. Based on this definition the deformation gradient is defined as

$$
\boldsymbol{F}=\nabla_{X} \varphi
$$

which maps infinitesimal referential line elements $\mathrm{d} \boldsymbol{X}$ onto their spatial counterparts $\mathrm{d} \boldsymbol{x}$. The Jacobian $J=\operatorname{det}(\boldsymbol{F})$ maps referential volume elements $\mathrm{d} V$ onto current volume elements $\mathrm{d} v$. Furthermore, the referential and spatial area normals are represented by $\mathrm{d} \boldsymbol{A}=\boldsymbol{N} \mathrm{d} A$ and $\mathrm{d} \boldsymbol{a}=\boldsymbol{n} \mathrm{d} a$, respectively. The transformation of infinitesimal area elements between the reference and the spatial configuration is defined by means of Nanson's formula $\boldsymbol{n} \mathrm{d} a=\operatorname{cof}(\boldsymbol{F}) \cdot \boldsymbol{N} \mathrm{d} A$, with the co-factor of $\boldsymbol{F}$ defined as

$$
\operatorname{cof}(\boldsymbol{F})=J \boldsymbol{F}^{-\mathrm{t}}
$$

Following Flory [63] and Ogden [64], the multiplicative composition of the deformation gradient is defined as

$$
\boldsymbol{F}=\left[J^{1 / 3} \boldsymbol{I}\right] \cdot \overline{\boldsymbol{F}}
$$

where $J^{1 / 3} \boldsymbol{I}$ and $\overline{\boldsymbol{F}}$ are the volumetric and the isochoric parts of the deformation gradient, respectively, and $\boldsymbol{I}$ is the second-order identity tensor. We also define the right and left 
Cauchy-Green tensors, $\boldsymbol{C}$ and $\boldsymbol{b}$, as well as their modified counterparts, $\overline{\boldsymbol{C}}$ and $\overline{\boldsymbol{b}}$, as

$$
\begin{gathered}
\boldsymbol{C}=\boldsymbol{F}^{T} \cdot \boldsymbol{F}=J^{2 / 3} \overline{\boldsymbol{C}}, \quad \overline{\boldsymbol{C}}=\overline{\boldsymbol{F}}^{T} \cdot \overline{\boldsymbol{F}} \\
\boldsymbol{b}=\boldsymbol{F} \cdot \boldsymbol{F}^{T}=J^{2 / 3} \overline{\boldsymbol{b}}, \quad \overline{\boldsymbol{b}}=\overline{\boldsymbol{F}} \cdot \overline{\boldsymbol{F}}^{T}
\end{gathered}
$$

Anisotropy directions are defined by means of a set of referential unit vectors $\boldsymbol{a}_{0}^{i}, i=1,2$ with $\left\|\boldsymbol{a}_{0}^{i}\right\|=1$, which account for two families of fibre reinforcements embedded in the continuum. Vector $\overline{\boldsymbol{a}}^{i}=\overline{\boldsymbol{F}} \cdot \boldsymbol{a}_{0}^{i}$ defines the spatial configuration of the $i$-th fibre family with $\left\|\overline{\boldsymbol{a}}^{i}\right\|>0$ denoting the stretch in the direction of the particular fibre family. Furthermore, a set of symmetric second-order tensors is introduced

$$
\begin{aligned}
\boldsymbol{H}^{i} & =\varkappa \boldsymbol{I}+[1-3 \varkappa] \boldsymbol{a}_{0}^{i} \otimes \boldsymbol{a}_{0}^{i}, \\
\overline{\boldsymbol{h}}^{i} & =\varkappa \overline{\boldsymbol{b}}+[1-3 \varkappa] \overline{\boldsymbol{a}}^{i} \otimes \overline{\boldsymbol{a}}^{i} .
\end{aligned}
$$

The parameter $\varkappa \in[0,1 / 3]$ accounts for the dispersion on the mean fibre orientation and measures the 'degree of anisotropy' in the material response due to the fibre reinforcements. When $\varkappa=0$ every material fibre in the $i$-th family of fibres is perfectly aligned along the direction defined by the referential vector $\boldsymbol{a}_{0}^{i}$, while for $\varkappa=1 / 3$ all fibres are evenly distributed and have no preferential orientation. For additional background information on the modeling on fibre-reinforced materials the reader is referred to, e.g., Spencer [65]; Weiss et al. [66]; Menzel and Steinmann [67]; Menzel et al. [68].

Furthermore, we define the following operators

$$
\begin{aligned}
\operatorname{tr}(\bullet) & =(\bullet): \boldsymbol{I}, \\
\operatorname{Tr}(\bullet) & =(\bullet): \boldsymbol{C}, \\
(\bullet) \odot(\bullet)_{I J K L} & =\frac{1}{2}\left[(\bullet)_{I K}(\bullet)_{J L}+(\bullet)_{I L}(\bullet)_{J K}\right], \\
\mathbb{I} & =\boldsymbol{I} \odot \boldsymbol{I}, \\
\mathbb{P} & =\mathbb{I}-\frac{1}{3} \boldsymbol{C}^{-1} \otimes \boldsymbol{C}, \\
\tilde{\mathbb{P}} & =\mathbb{I}-\frac{1}{3} \boldsymbol{I} \otimes \boldsymbol{I},
\end{aligned}
$$


and finally, the following modified invariants

$$
\begin{aligned}
\bar{I}_{1} & =\overline{\boldsymbol{C}}: \boldsymbol{I}=\overline{\boldsymbol{b}}: \boldsymbol{I}=J^{-2 / 3} I_{1}, \\
\bar{I}_{4} & =\boldsymbol{H}^{1}: \overline{\boldsymbol{C}}=\boldsymbol{h}^{1}: \overline{\boldsymbol{I}} \\
\bar{I}_{6} & =\boldsymbol{H}^{2}: \overline{\boldsymbol{C}}=\boldsymbol{h}^{2}: \overline{\boldsymbol{I}} \\
\bar{E}^{1} & =\bar{I}_{4}-1, \\
\bar{E}^{2} & =\bar{I}_{6}-1,
\end{aligned}
$$

are introduced. Here, $\bar{E}^{i}$ is a strain-like quantity that measures the homogenised stretch of the $i$-th fibre family. From equations (2.7), (2.15) and (2.16) we observe that when $\varkappa=1 / 3$, i.e. when fibres are evenly distributed, $\bar{I}_{4}=\bar{I}_{6}=\bar{I}_{1}$ and complete isotropy is obtained.

\subsection{Hyperelasticity}

In the following we introduce the basic equations that define the framework of the constitutive modelling of hyperelastic materials. Two cases are differentiated. On the one hand, the case where the material behaves elastically, and on the other hand the case were a reduction in the load-bearing capacity of the material occurs as a consequence of damage-related phenomena.

\subsubsection{Hyperelasticity with no internal dissipation}

The fundamental assumption in hyperelasticity is that it exits a functional $\Psi$ called strain energy function, or simply strain energy, which is defined per unit reference volume. In this work we make use of a decoupled representation of $\Psi$ that follows from the kinematic assumption (2.3), hence

$$
\Psi(\boldsymbol{C}, \boldsymbol{H})=\Psi^{\mathrm{vol}}(J)+\Psi^{\mathrm{ich}}(\overline{\boldsymbol{C}}, \boldsymbol{H}),
$$

where $\Psi^{\mathrm{vol}}$ and $\Psi^{\mathrm{ich}}$ represent the volumetric and isochoric responses of the material under consideration, respectively. Following the Coleman-Noll procedure Coleman and Noll [69]; Coleman and Gurtin [70] the Clausius-Planck form of the second law of thermodynamics is written

$$
\begin{aligned}
\mathcal{D}_{\mathrm{INT}} & =\boldsymbol{S}: \frac{1}{2} \dot{\boldsymbol{C}}-\dot{\Psi}, \\
& =\left[\frac{1}{2} \boldsymbol{S}-\frac{\partial \Psi}{\partial \boldsymbol{C}}\right]: \dot{\boldsymbol{C}},
\end{aligned}
$$


which for a perfectly elastic material, i.e. $\mathcal{D}_{\text {INT }}=0$, allows to relate the internal forces in the material with its deformation in the following way

$$
\boldsymbol{S}=2 \frac{\partial \Psi(\boldsymbol{C}, \boldsymbol{H})}{\partial \boldsymbol{C}}
$$

where $\boldsymbol{S}$ is the second Piola-Kirchhoff stress tensor. Furthermore, $\boldsymbol{S}$ can equally be expressed as an additive contribution of a volumetric and an isochoric contribution

$$
\begin{aligned}
\boldsymbol{S} & =\boldsymbol{S}^{\mathrm{vol}}+\boldsymbol{S}^{\mathrm{ich}}, \\
\boldsymbol{S}^{\mathrm{vol}} & =2 \frac{\partial \Psi^{\mathrm{vol}}(J)}{\partial \boldsymbol{C}}=J p \boldsymbol{C}^{-1}, \\
\boldsymbol{S}^{\mathrm{ich}} & =2 \frac{\partial \Psi^{\mathrm{ich}}(\overline{\boldsymbol{C}})}{\partial \boldsymbol{C}}=J^{-2 / 3} \mathbb{P}: \overline{\boldsymbol{S}},
\end{aligned}
$$

where the hydrostatic pressure, $p$, and the fictitious second Piola-Kirchhoff stress tensor, $\overline{\boldsymbol{S}}$, are defined as:

$$
\begin{gathered}
p=\frac{\mathrm{d} \Psi^{\mathrm{vol}}(J)}{\mathrm{d} J} \\
\overline{\boldsymbol{S}}=2 \frac{\partial \Psi^{\mathrm{ich}}(\overline{\boldsymbol{C}})}{\partial \overline{\boldsymbol{C}}},
\end{gathered}
$$

Finally, the Piola transformation gives us the Cauchy stress tensor

$$
\begin{aligned}
\boldsymbol{\sigma} & =\boldsymbol{\sigma}^{\mathrm{vol}}+\boldsymbol{\sigma}^{\mathrm{ich}} \\
\boldsymbol{\sigma}^{\mathrm{vol}} & =p \boldsymbol{I} \\
\boldsymbol{\sigma}^{\mathrm{ich}} & =J^{-1} \overline{\boldsymbol{F}}[\mathbb{P}: \overline{\boldsymbol{S}}] \overline{\boldsymbol{F}}^{T} .
\end{aligned}
$$

\subsubsection{Hyperelasticity with damage}

We consider now the case where the inelastic phenomena associated to damage reduce the load-bearing capacity of the material. A reduction factor $1-d$, initially proposed by Kachanov [28], is used to weight the strain energy. The internal variable $d \in[0,1]$, also known as damage variable, is a scalar term that quantifies the reduction in the load-bearing capacity of the material. For an intact material $d=0$, while for a fully damaged material $d=1$. Furthermore, as proposed by Simo [71], damage affects only the isochoric term, hence the strain energy takes the form

$$
\Psi(\boldsymbol{C}, \boldsymbol{H})=\Psi^{\mathrm{vol}}(J)+[1-d] \Psi^{\mathrm{ich}}(\overline{\boldsymbol{C}}, \boldsymbol{H})
$$


Again, following the Coleman-Noll procedure, the Clausius-Planck form of the second law of thermodynamics is written as

$$
\begin{aligned}
\mathcal{D}_{\mathrm{INT}} & =\boldsymbol{S}: \frac{1}{2} \dot{\boldsymbol{C}}-\dot{\Psi} \\
& =\left[\frac{1}{2} \boldsymbol{S}-\frac{\partial \Psi^{\mathrm{vol}}}{\partial \boldsymbol{C}}-[1-d] \frac{\partial \Psi^{\mathrm{ich}}}{\partial \boldsymbol{C}}\right]: \dot{\boldsymbol{C}}-\dot{d} \Psi
\end{aligned}
$$

and then, using equations (2.24) and (2.25), the second Piola-Kirchhoff stress and the non-negative internal dissipation are given as

$$
\begin{aligned}
\boldsymbol{S} & =\boldsymbol{S}^{\mathrm{vol}}+[1-d] \boldsymbol{S}^{\mathrm{ich}}, \\
\mathcal{D}_{\mathrm{INT}} & =\dot{d} \Psi \geqslant 0 .
\end{aligned}
$$

Finally, the Piola transformation gives us the Cauchy stress tensor

$$
\boldsymbol{\sigma}=\boldsymbol{\sigma}^{\mathrm{vol}}+[1-d] \boldsymbol{\sigma}^{\mathrm{ich}}
$$

\subsection{Constitutive modelling of a soft fibre-reinforced biological tissue}

As an special consideration related to the case of fibre-reinforced soft tissues, we particularize equation (2.31) in this thesis by assuming that the isochoric contribution of the strain energy function is composed of three phases $i=\{0,1,2\}$, each representing one of the main load-bearing constituents of the arterial tissue, i.e. the isotropic ground substrate, $i=0$, and the two collagen fibre-families $i=\{1,2\}$. The strain energy function then takes the form

$$
\Psi\left(\boldsymbol{F}, \boldsymbol{H}^{i}, \mathrm{~d}^{i}\right)=\Psi^{\mathrm{vol}}(J)+\left[1-d^{0}\right] \Psi^{0}(\overline{\boldsymbol{F}})+\sum_{i=1,2}\left[1-d^{i}\right] \Psi^{i}\left(\overline{\boldsymbol{F}}, \boldsymbol{H}^{i}\right),
$$

where the reduction factor accounting for the damage-related effects is defined on each phase individually by means of individual damage variables $d^{i} \in[0,1]$.

Remark 2.3.1 From equation (4.1) on, the upper index ich will no longer be used to differentiate the isochoric and volumetric contributions from the strain energy function. Each individual contribution $\Psi^{i}$ of phases $i=\{0,1,2\}$ is assumed to be an isochoric contribution.

In the present work we adopt the hyperelastic model proposed by Gasser et al. [7]. This particular constitutive model regards the isotropic matrix as an incompressible neo-Hookean 
material, hence the following contributions are defined

$$
\begin{aligned}
\Psi^{\mathrm{vol}} & =\frac{\kappa_{\mathrm{e}}}{2}[J-1]^{2}, \\
\Psi^{0} & =\frac{\mu_{\mathrm{e}}}{2}\left[\bar{I}_{1}-3\right],
\end{aligned}
$$

where the elastic constants are represented by the Lamé-parameters in terms of the shear modulus $\mu_{\mathrm{e}}$ and the bulk modulus $\kappa_{\mathrm{e}}$. The particular format of $\Psi^{\mathrm{vol}}$ has been chosen to enforce the incompressibility constraint $J-1=0$, hence $\kappa_{\mathrm{e}}$ can be interpreted as a penalty parameter. The anisotropic contribution of the local free energy is based on a modified version of an orthotropic exponential model with two families of fibres, as originally proposed by Holzapfel et al. [72], including the effect of fibre dispersion, see Gasser et al. [7], the review article by Holzapfel and Ogden [73] or Menzel et al. [74]. The free energy adopted is represented by the exponential format

$$
\Psi^{i}=\frac{k_{1}^{i}}{2 k_{2}^{i}}\left[\exp \left(k_{2}^{i}\left\langle\bar{E}^{i}\right\rangle^{2}\right)-1\right]
$$

for $i=1,2$. The term $\left\langle\bar{E}^{i}\right\rangle$, where $\langle\bullet\rangle=[|\bullet|+\bullet] / 2$ is the Macaulay bracket, reflects the basic assumption that fibres can support tension only. Consequently, the anisotropic strain energy density $\Psi^{i}$ only contributes if the fibre-related strain is positive, i.e. $\bar{E}^{i}>0$.

The total Cauchy stress tensor can be derived by means of the constitutive equation for hyperelastic materials, i.e. equations (2.25) and (2.30), and takes the same additive structure as (4.1), i.e.

$$
\boldsymbol{\sigma}=\boldsymbol{\sigma}^{\mathrm{vol}}+\left[1-d^{0}\right] \boldsymbol{\sigma}^{0}+\sum_{i=1,2}\left[1-d^{i}\right] \boldsymbol{\sigma}^{i} .
$$

The Cauchy stress tensor associated to the volumetric deformation is derived from the functional (2.37) and reads

$$
\boldsymbol{\sigma}^{\mathrm{vol}}=k_{\mathrm{e}}[J-1] \boldsymbol{I}
$$

while the elastic Cauchy stresses associated to the isochoric deformation are defined for each of the phases as

$$
\begin{aligned}
\boldsymbol{\sigma}^{0} & =\mu_{\mathrm{e}} J^{-1}\left[\overline{\boldsymbol{b}}-\left[\bar{I}_{1} / 3\right] \boldsymbol{I}\right] \\
\boldsymbol{\sigma}^{i} & =2 J^{-1} k_{1}^{i} \bar{E}^{i} \exp \left(k_{2}^{i}\left\langle\bar{E}^{i}\right\rangle^{2}\right)\left[\overline{\boldsymbol{h}}^{i}-\left[\bar{E}^{i} / 3\right] \boldsymbol{I}\right] \quad i=1,2 .
\end{aligned}
$$

Remark 2.3.2 The hydrostatic term of the Cauchy stress tensor specified in equation (2.41) is further modified when a multivariational formulation is used in section 4.3.4. 


\section{Regularised local continuum damage model based on the mesoscopic scale for fibred biological tissues}

In this chapter we present a phenomenological continuous damage model with regularised softening for soft tissues, along with meso-structurally based definitions for its material parameters. Material properties of soft fibrous tissues are highly conditioned by the hierarchical structure of this kind of composites. Collagen based tissues present, at decreasing length scales, a complex framework of fibres, fibrils, tropocollagen molecules and aminoacids. Following this consideration, in this section we derive a relationship between the mechanical and geometrical properties of the fibril constituents and the soft tissue material parameters at macroscopic scale. A Hodge-Petruska two-dimensional model is used to describe the fibrils as staggered arrays of tropocollagen molecules. After a mechanical characterisation of each of the fibril components, two fibril failures modes related with two planes of weakness are found. This chapter is concluded with numerical analysis at fibril, fibre and tissue levels to show the capabilities of the model.

\subsection{Local damage model with regularised softening}

Soft tissues have a hierarchical structure with several scales, from the amino-acids forming the proteins in the atomistic scale to the arterial walls in the continuum scale. The elementary building block of the fibrous reinforcement in soft tissues is collagen. This structural protein consists of tropocollagen (TC) molecules with an aspect ratio close to 200. TC molecules are disposed in staggered arrays forming fibrils which are organised in families of fibres surrounded by an almost incompressible ground matrix (see [75], [76], [18] and [20] among others).

Here, we present a material damage model suited for fibrous materials. As stated in [77], this kind of continuous damage models can reproduce the Mullins' effect only after damage 
initiation. Two main hypothesis have been considered: the damage processes depend only on the isochoric deformation and each material phase, ground matrix and fibres, damages independently. The model presented here needs only three parameters to characterise the material softening in each phase of the composite: a threshold value that defines the initial size of the elastic domain (usually a limit value of the Cauchy stress or the stretch in the uniaxial homogeneous tension test), a parameter that defines the total amount of internal dissipated energy and a coefficient that affects the rate of softening.

\subsubsection{Ingredients of the local damage model}

As proposed by Simo [71], we assume that damage affects only the isochoric term of the strain energy function, hence damage mechanisms are associated only with isochoric processes and therefore are independent from hydrostatic pressure. Furthermore, as an special consideration related to the case of fibre-reinforced soft tissues, we assume that the isochoric contribution of the strain energy function is composed of three phases $i=\{0,1,2\}$, each representing one of the main load-bearing constituents of the arterial tissue, i.e. the isotropic ground substrate, $i=0$, and the two collagen fibre-families $i=\{1,2\}$. A particularisation of the internal dissipation obtained in equations (2.32) and (2.34) for the case in which damage evolves independently in each phase, gives

$$
\mathcal{D}_{\mathrm{INT}}=\dot{d}^{g} \Psi^{0}+\dot{d}^{1} \Psi^{1}+\dot{d}^{2} \Psi^{2} \geq 0
$$

which allows us to define $\Psi^{i} \geq 0$ as the thermodynamic variable conjugate to $d^{i}$, with $i=\{0,1,2\}$. Non-negativeness of internal dissipation implies the damage variables to be non-decreasing, leading to the following condition:

$$
d^{i} \in[0,1], \quad \dot{d}^{i} \geq 0 \quad \text { with } i=\{0,1,2\}
$$

Following the model proposed by [78]-[79] we introduce for each phase the notion of equivalent strain $\bar{\tau}^{i}$ as the following norm:

$$
\bar{\tau}^{i}=\sqrt{2 \Psi^{i}(\overline{\boldsymbol{C}})}
$$

We introduce also a set of damage surfaces $\Phi^{i}=0$, with $i=\{0,1,2\}$, in the strain space that allows to define a set of damage criteria imposing, at any time $t \in \mathbb{R}^{+}$of the loading 
process,

$$
\begin{aligned}
& \Phi^{0}\left(\bar{I}_{1}, \bar{I}_{2}, r^{0}\right)=\sqrt{2 \Psi^{0}}-r^{0} \leq 0 \\
& \Phi^{1}\left(\bar{I}_{1}, \bar{I}_{4}, r^{1}\right)=\sqrt{2 \Psi^{1}}-r^{1} \leq 0 \\
& \Phi^{2}\left(\bar{I}_{1}, \bar{I}_{6}, r^{2}\right)=\sqrt{2 \Psi^{2}}-r^{2} \leq 0 .
\end{aligned}
$$

Here $r^{i}$ is the strain-like internal variable that defines the damage threshold at the current configuration satisfying $r^{i} \geq r_{0}^{i}$, with $r_{0}^{i}$ the initial damage threshold.

Equations (3.4)-(3.6) define the elastic domain of the material response. Damage evolution must satisfy the complementary Kuhn-Tucker loading/unloading conditions:

$$
\dot{r}^{i} \geq 0, \quad \Phi^{i} \leq 0 \quad \text { and } \quad \dot{r}^{i} \Phi^{i}=0
$$

as well as the damage consistency condition during persistent damage:

$$
\dot{r}^{i} \dot{\Phi}^{i}=0
$$

Denoting the normal to the damage surfaces $\boldsymbol{N}^{i}:=\partial \Phi^{i} / \partial \overline{\boldsymbol{C}}$ the following alternative situations may occur: either

$$
\Phi^{i}<0 \text { or } \Phi^{i}=0 \text { and }\left\{\begin{array}{l}
\boldsymbol{N}^{i}: \delta \overline{\boldsymbol{C}}<0 \\
\boldsymbol{N}^{i}: \delta \overline{\boldsymbol{C}}=0 \\
\boldsymbol{N}^{i}: \delta \overline{\boldsymbol{C}}>0
\end{array}\right.
$$

where $\delta \overline{\boldsymbol{C}}$ stands for an admissible variation of the deviatoric strain tensor.

The model thus defined admits a closed integration of the rate of the internal variable $r^{i}$, being able to be integrated over time as:

$$
r^{i}(t)=\max _{\tau \in[0, t]}\left[r_{0}^{i}, \sqrt{2 \Psi^{i}(\tau)}\right] .
$$

We can also define a stress-like internal variable $q^{i}$ whose rate of change is expressed with the following hardening rule (with $i=\{0,1,2\}$ ):

$$
\dot{q}^{i}=H^{i}\left(r^{i}\right) \dot{r}^{i}, \quad H^{i}\left(r^{i}\right)=\frac{\partial q^{i}}{\partial r^{i}}, \quad q_{0}^{i}=r_{0}^{i}
$$

where $H^{i}$ is the softening/hardening modulus, such that $H^{i}\left(r^{i}\right)>0$ if there is strain hardening and $H^{i}\left(r^{i}\right)<0$ if there is strain softening, and $r_{0}^{i}$ is the parameter that defines the initial size of the elastic domain. Damage parameters are finally expressed in terms of 
the previously introduced internal variables as:

$$
d^{0}=1-\frac{q^{0}\left(r^{0}\right)}{r^{0}}, \quad d^{1}=1-\frac{q^{1}\left(r^{1}\right)}{r^{1}}, \quad d^{2}=1-\frac{q^{2}\left(r^{2}\right)}{r^{2}}
$$

\subsubsection{Internal dissipation and softening regularisation}

We can particularise the value of the internal dissipation given in expression (3.1), after some algebraic manipulations, as

$$
\mathcal{D}_{\mathrm{INT}}=\sum_{i=0,1,2} \dot{d}^{i} \Psi^{i}=\sum_{i=0,1,2} \frac{1}{2}\left[q^{i} \dot{r}^{i}-\dot{q}^{i} r^{i}\right]
$$

where the equations (3.3) and (3.11) have been used.

We assume hereinafter our material undergoes strain softening. The rate of change of the stress-like internal variable $\dot{q}^{i}$, with $i=\{0,1,2\}$, can be defined as:

$$
\dot{q}^{i}=-H^{i}\left(q^{i}\right) \dot{r}^{i}=-A^{i} q^{i, \chi} \dot{r}^{i}
$$

where $-H^{i}$ is the softening modulus, $\chi$ is a parameter that affects the rate of softening, and $A^{i}$ is a value to be defined by imposing the objectivity of the approach regarding the characteristic size of the mesh in a finite element analysis [80].

We reproduce damage in this work following the principles of the so called smeared crack models [81] where inelastic phenomena are considered to be distributed inside the finite element. If our model has to be insensitive to the mesh size, the softening modulus $H^{i}$ we use at each integration point has to be regularised (see [82] for a full description) in terms of the material parameter surface density of dissipation energy and a characteristic size of the finite element [83].

Let us define a subdomain $\Omega^{h} \in \mathcal{B}_{0}$ where inelastic processes take place. The volume of this subdomain, here we are assuming it is a finite element, can be defined as $\int_{\Omega^{h}} \mathrm{~d} \Omega=S h$, where $S$ is an averaged cross section and $h$ is its characteristic size (see Figure 3.1).

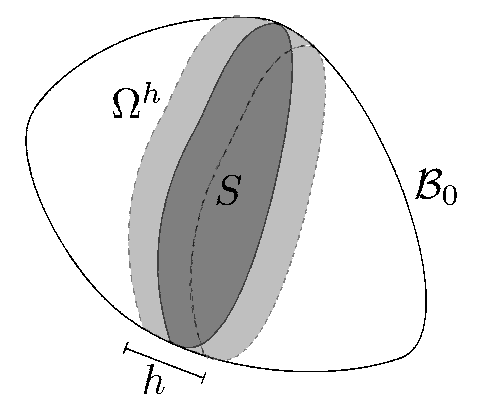

Figure 3.1: Damaged band $\Omega^{h}$ in a continuum domain. 
We assume there is a monotonic loading process and the material undergoes strain softening, so the total dissipated energy $\Psi_{\text {Tот }}$ until material exhaustion is:

$$
\begin{aligned}
\Psi_{\mathrm{TOT}} & =\int_{\Omega^{h}} \mathrm{~d} \Omega\left[\int_{t=t_{d}}^{\infty} \mathcal{D}_{\mathrm{INT}} \mathrm{d} t\right] \\
& =\int_{\Omega^{h}} \mathrm{~d} \Omega\left[\int_{t=t_{d}}^{\infty} \sum_{i=0,1,2} \frac{1}{2}\left[q^{i} \dot{r}^{i}-\dot{q}^{i} r^{i}\right] \mathrm{d} t\right] \\
& =\sum_{i=0,1,2} \int_{\Omega^{h}} \mathrm{~d} \Omega\left[\int_{t=t_{d}}^{\infty} \frac{1}{2}\left[q^{i} \dot{r}^{i}-\dot{q}^{i} r^{i}\right] \mathrm{d} t\right]
\end{aligned}
$$

If we impose, for each phase $i=\{0,1,2\}$, that the total dissipated energy is given by the material parameter surface density of dissipated energy $G^{i, f}$, after some algebraic manipulations in equation (3.15) and using expression (3.14) we arrive to:

$$
\Psi_{\text {тот }}=\sum_{i=0,1,2} \int_{\Omega^{h}} \mathrm{~d} \Omega\left[\frac{1}{A^{i}} \frac{\left[q_{0}^{i}\right]^{2-\chi}}{(2-\chi)}\right]=\sum_{i=0,1,2} S h\left[\frac{1}{A^{i}} \frac{\left[q_{0}^{i}\right]^{2-\chi}}{[2-\chi]}\right]=\sum_{i=0,1,2} S G^{f, i}
$$

which allows us to obtain the expression for $A^{i}$ in equation (3.14) as:

$$
A^{i}=\frac{\left[q_{0}^{i}\right]^{2-\chi}}{[2-\chi]} \frac{1}{G^{f, i}} h
$$

Finally, we obtain the regularised softening modulus as

$$
H^{i}\left(q^{i}(t), h\right)=-\frac{\left[q_{0}^{i}\right]^{2-\chi}}{[2-\chi]} \frac{1}{G^{f, i}} q^{\chi, i}(t) h
$$

where $h$, in case the domain has been discretised by finite elements, is the characteristic length of this element [83].

\subsubsection{Summary of material parameters}

In this model, for each material phase $i=\{0,1,2\}$ we need to characterise the effective strain energy function $\Psi^{i}$, the initial size of the elastic domain $r_{0}^{i}$, the surface density of dissipated energy $G^{f, i}$ and the rate of softening $\chi$. The total amount of material parameters needed is summarised in Table 3.1. 


\begin{tabular}{|c|c|c|c|c|}
\hline \multirow{2}{*}{$\begin{array}{l}\text { Component } \\
\text { Ground matrix }\end{array}$} & \multicolumn{2}{|c|}{ Elastic behaviour } & \multicolumn{2}{|r|}{ Inelastic behaviour } \\
\hline & $\mu_{\mathrm{e}}[\mathrm{Pa}]$ & Shear modulus & $\begin{array}{c}\sigma_{Y}^{0}[\mathrm{~Pa}] \\
\mathcal{G}^{f, 0}[\mathrm{~N} / \mathrm{m}] \\
\chi^{0}[-]\end{array}$ & $\begin{array}{l}\text { Stress elastic threshold in the } \\
\text { uniaxial homogeneous tension test. } \\
\text { surface density of dissipated } \\
\text { energy. } \\
\text { Rate of softening. }\end{array}$ \\
\hline $\begin{array}{l}\text { Family } i \text { of fibres } \\
i=\{1,2\}\end{array}$ & $\begin{array}{c}k_{1}^{i}[\mathrm{~Pa}] \\
k_{2}^{i}[-]\end{array}$ & $\begin{array}{l}\text { Elastic constant } \\
\text { Elastic constant }\end{array}$ & $\begin{array}{c}\sigma_{Y}^{i}[\mathrm{~Pa}] \\
\mathcal{G}^{f, i}[\mathrm{~N} / \mathrm{m}] \\
\chi^{i}[-]\end{array}$ & $\begin{array}{l}\text { Stress elastic threshold in the } \\
\text { uniaxial homogeneous tension test. } \\
\text { surface density of dissipated } \\
\text { energy. } \\
\text { Rate of softening. }\end{array}$ \\
\hline Tissue & $\kappa_{\mathrm{e}}[\mathrm{Pa}]$ & Bulk modulus & & - \\
\hline
\end{tabular}

Table 3.1: Material parameters.

\subsection{Mesoscopic characterisation of fibre inelastic be- haviour}

In this study we derive the macroscopic material parameters $\left\{k_{1}^{i}, k_{2}^{i}, \sigma_{Y}^{i}, \mathcal{G}^{f, i}\right\}$ for the family of fibres $i$ from the mechanical properties of its mesoscopic constituents. We have assumed a simplified hierarchical structure of soft tissues such that we consider soft tissues as a fibre reinforced composite and fibres as a fibril reinforced composite. Fibrils, assumed as a staggered array of tropocollagen molecules, is the smallest scale we have considered (see Fig. 3.2).

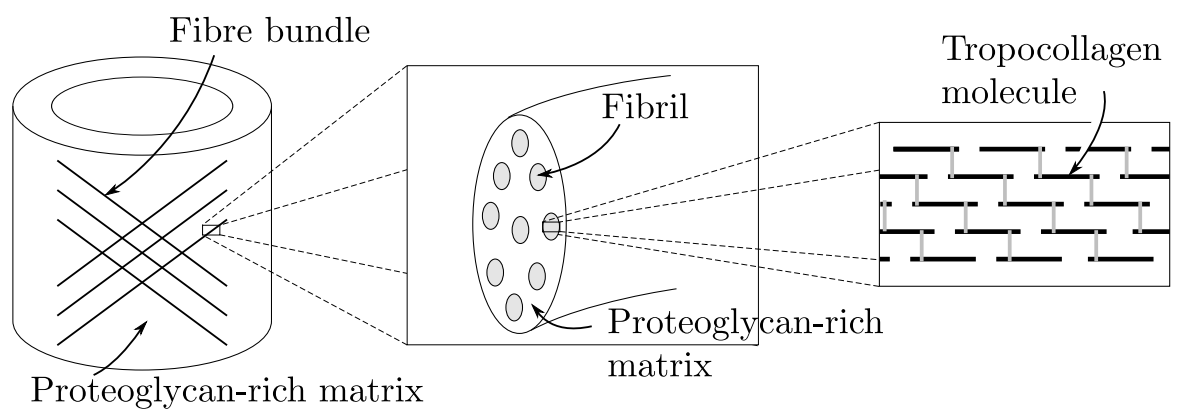
(a) Tissue as a fiber
(b) Fibre as a fibril reinforced composite
reinforced composite
(c) Fibril as a staggered array of TC molecules

Figure 3.2: Hierarchical structure of soft tissues

This section is summarised as follows. Firstly, we obtain the fibril elastic stretch threshold considering that its reference configuration has a wavy arrangement. Afterwards we study the mesomechanical behaviour of the fibril: we introduce the two-dimensional HodgePetruska model [84], we define the mechanical features of each component of the fibril and 
we characterise the elastic/inelastic mesomechanical behaviour of the fibril as a whole. Finally, we obtain the macromechanical behaviour of the fibres by homogenising the properties of the fibrils and the proteoglycan-rich ground matrix.

\subsubsection{Fibril mesoscopic model}

Fibril elastic stretch threshold The wavy structure of the fibrils has an important bearing on the mechanical properties of soft tissues. It affects both the evolution of the tension stiffening displayed in the uniaxial tension test and the value of the elastic stretch threshold (assuming the crimped configuration of the fibril is its reference configuration). We consider a crimped fibril displaying a sinusoidal shape [12] characterised by the dimensionless parameter $r$ (see Fig. 3.3a).

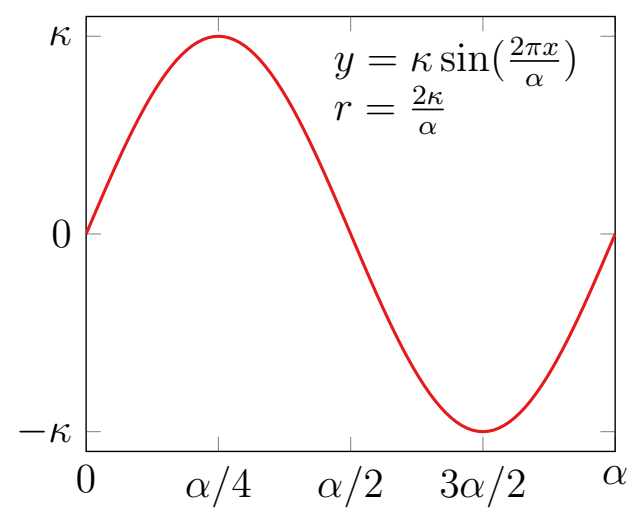

(a)

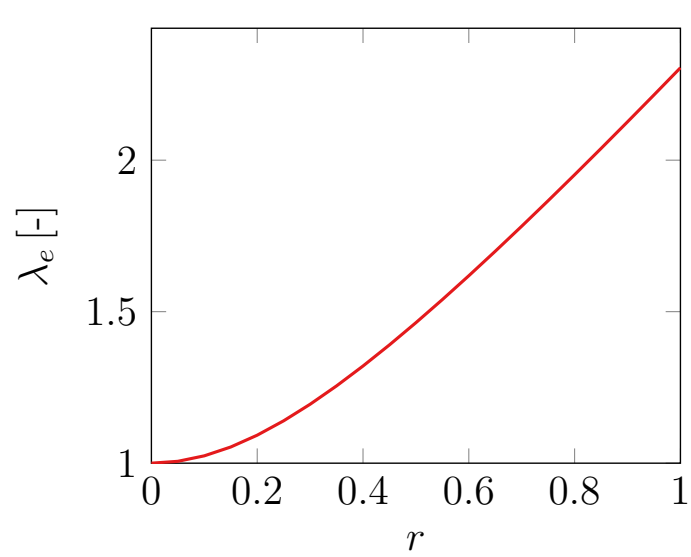

(b)

Figure 3.3: (a) 1-D model of a wavy fibril. (b) Dependence $\lambda_{e}=\lambda_{e}(r)$.

The total length $L$ can be expressed as:

$$
L=\frac{2 \alpha}{\pi} \sqrt{1+\pi^{2} r^{2}} \underbrace{\mathrm{E}\left[\frac{\pi^{2} r^{2}}{1+\pi^{2} r^{2}}\right]}_{E(m)}
$$

where $\mathrm{E}(m)$ is the complete elliptic integral of the second kind. We assume as a hypothesis that (a) the onset of damage occurs when the fibril is fully stretched [85] and (b) both fibril and fibre share the same elastic stretch threshold $\lambda_{e}$. In this case we obtain the elastic threshold of the stretch for the fibres as:

$$
\lambda_{e}=\frac{L}{\alpha}=\frac{2}{\pi} \sqrt{1+\pi^{2} r^{2}} \mathrm{E}\left[\frac{\pi^{2} r^{2}}{1+\pi^{2} r^{2}}\right]
$$

whose dependence $\lambda_{e}=\lambda_{e}(r)$ is described in Fig. 3.3b. 
The two dimensional Hodge-Petruska model Following [18] and [24], a two dimensional Hodge-Petruska arrangement [84] has been considered to reproduce the staggered structure of the tropocollagen molecules inside a fibril. In this model, see Fig. 3.4, tropocollagen molecules (TC) are considered as only-tension bearing line elements displaying a staggered structure. The length $l$ of each TC molecule is around $300 \mathrm{~nm}$ and its diameter $d$ is around $1.5 \mathrm{~nm}$, the distance $h$ of stagger is around $67 \mathrm{~nm}$ and the gap $s$ between the ends of successive molecules is around $40 \mathrm{~nm}$ [12]. Finally, distance $D$ stands for the equilibrium distance between tropocollagen molecules, which has been considered as equal to the microfibril size of 4-20 nm measured in [86].

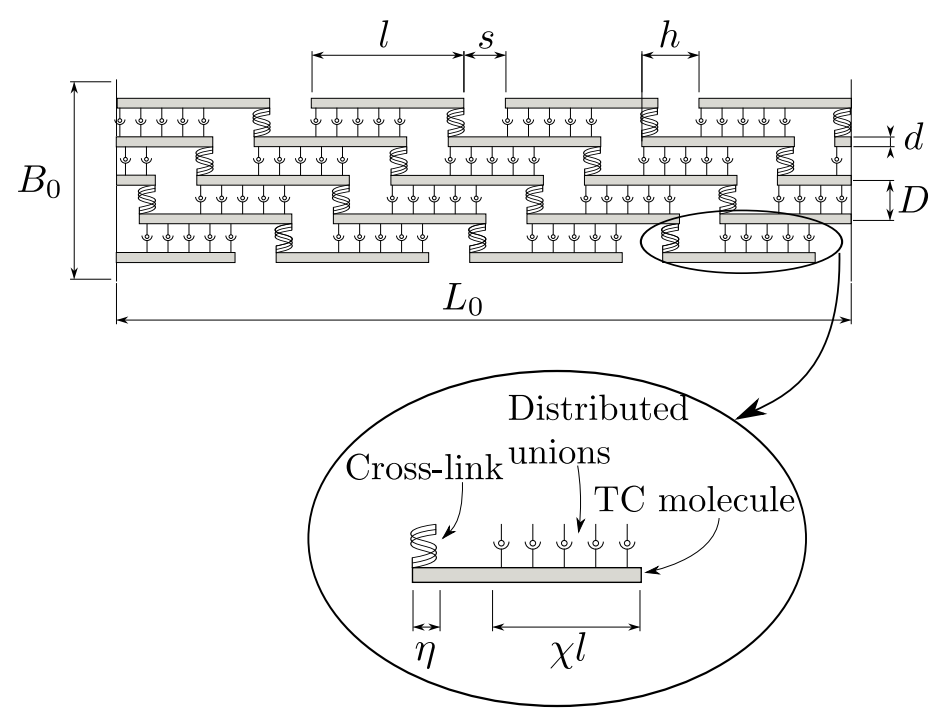

Figure 3.4: 2-D Hodge-Petruska model

Intermolecular adhesion, and therefore geometrical stability of the structure, is accomplished by the presence of (a) intermolecular distributed adhesive forces along the TC molecules and (b) localised cross-links at their ends [23; 87]. The intermolecular adhesive forces are distributed along the overlapped TC molecular length $\chi l$ and the localised cross links along $\eta=60 \AA$ at the end of the TC molecules [23]. These cohesive forces between TC molecules and the intermolecular bonds within them provides the inner response against an external solicitation. Table 3.2 summarises the proposed geometrical parameters of the Hodge-Petruska model used in this work.

Fibril mesomechanical model in elastic regimen We assume as a hypothesis that when a fibril is subjected to axial tension all TC molecules are loaded uniformly. The individual behaviour of each TC molecule can be simplified according to the scheme displayed in Fig. 3.5. In this model the stress supported by the TC molecule is transmitted to adjacent molecules through (a) the distributed unions along the overlapped molecular length and (b) the localised cross-links at its end. 


\begin{tabular}{lcc}
\hline Description & Symbol & Value \\
\hline Waviness parameter & $r$ & 0.33 \\
TC molecular length & $l$ & $300 \mathrm{~nm}^{(\mathrm{a})}$ \\
TC molecular diameter & $d$ & $1.5 \mathrm{~nm}^{(\mathrm{a})}$ \\
Gap between TC molecules & $s$ & $40 \mathrm{~nm}^{(\mathrm{a})}$ \\
Distance of stagger & $h$ & $67 \mathrm{~nm}^{(\mathrm{b})}$ \\
Equilibrium distance between & & \\
TC molecules & $D$ & $8 \mathrm{~nm}^{(\mathrm{c})}$ \\
Cross-link concentration length & $\eta$ & $6 \mathrm{~nm}^{(\mathrm{d})}$ \\
\hline${ }^{(\mathrm{a})}$ [88]. \\
(b) $[89]$. \\
(c) Microfibril size of 4-20 $\mathrm{nm}$ in [86]. \\
(d) [18].
\end{tabular}

Table 3.2: Geometrical properties of fibril components.

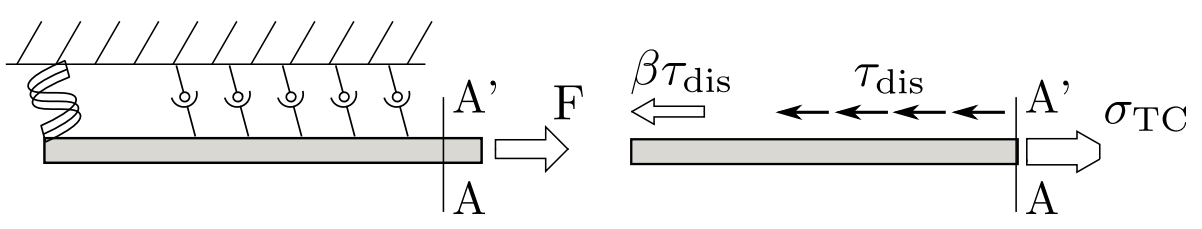

Figure 3.5: Unidimensional equilibrium of the TC molecule

Under quasi-static conditions, the tension in the TC molecule must be balanced by the strength provided in the distributed unions and in the cross-links (see Fig. 3.5). This scheme of forces can be interpreted as a series arrangement of the TC molecule with a parallel arrangement of the unions between TC molecules (see Fig. 3.6a).

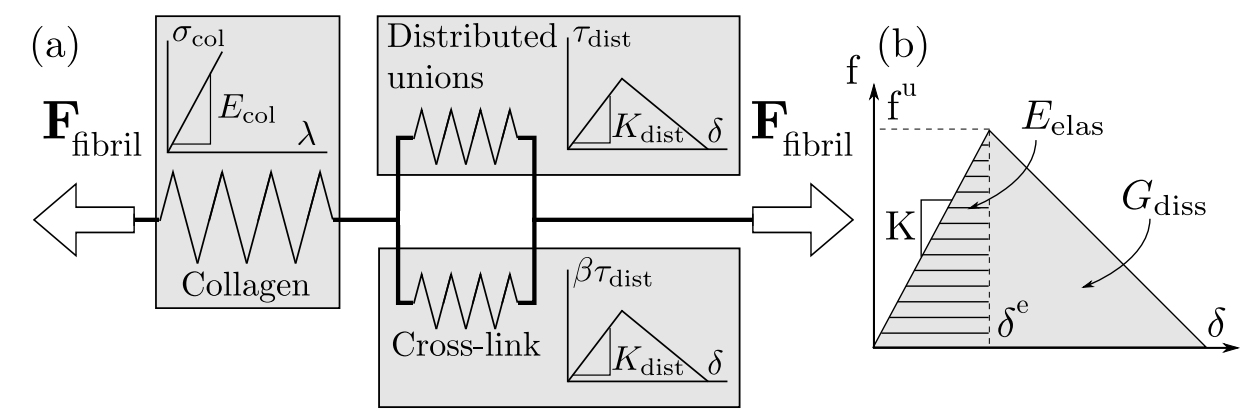

Figure 3.6: (a) Idealised mechanical model. (b) Mechanical characterisation of individual components.

We need to characterise the mesomechanical behaviour of each of the ingredients included in the model (TC molecule, distributed unions and cross-links). If we assume a mesoscopic linear behaviour, we have to define for each ingredient only three of the parameters shown in Fig. 3.6b. Table 3.3 summarises the reference values of the parameters elastic stiffness, 


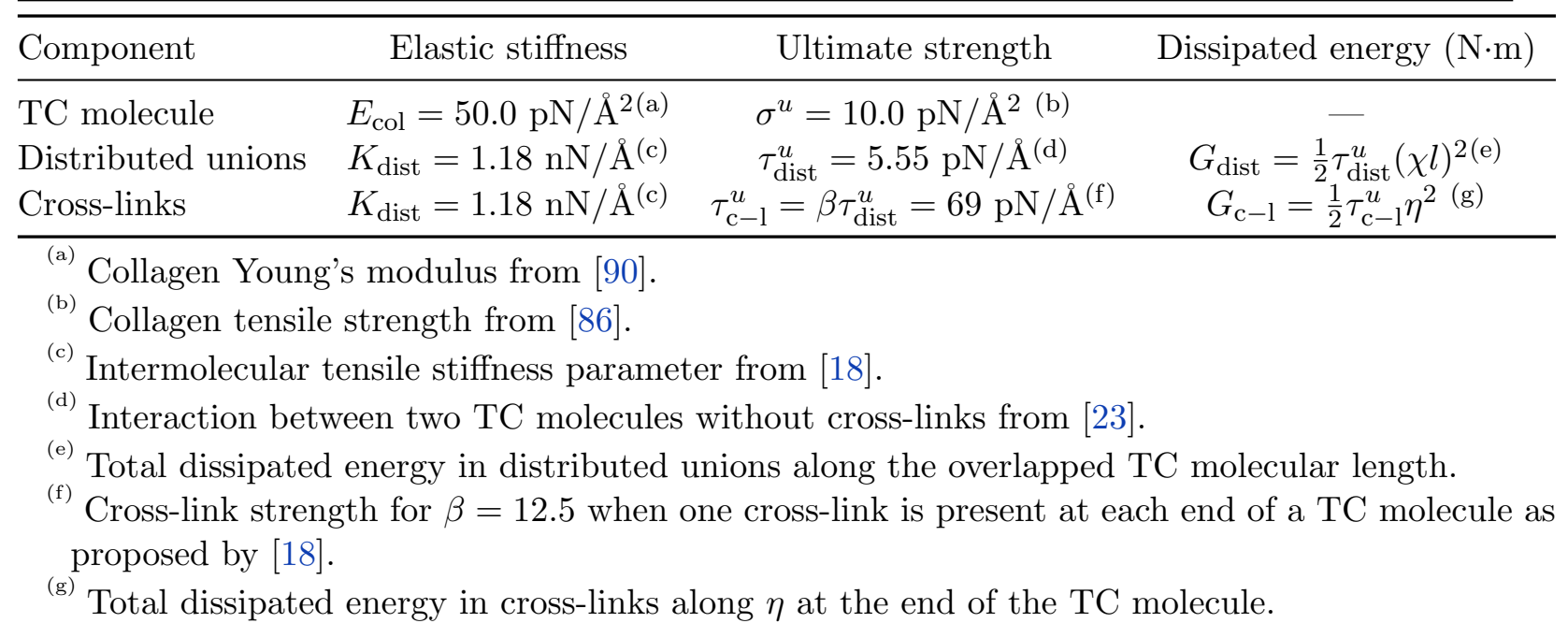

Table 3.3: Mesomechanical properties of fibril components.

ultimate strength and dissipated energy used in this work. Here we have considered the dissipated energy as the work required to exhaust the bearing capacity of the element.

Fibril failure modes We associate the onset of inelastic processes in the tissue with the beginning of degradation in the fibril components. If we study the mechanical idealisation of the TC molecule shown in Fig. 3.5, loss of bearing capacity can be related either with the failure of the collagen chains or with the failure of the adherent unions between TC molecules. Due to the high tensile strength of the collagen chains [86] we assume structural failure is associated only with the loss of cohesion at the unions between TC molecules.

In this work we propose as a hypothesis that there are two possible failure mechanisms associated with the loss of cohesion at the unions between TC molecules. Failure mechanisms depend on the relationship between the maximum cohesion strength $\eta \beta \tau_{\text {dis }}^{u}$ provided by the cross-links and the maximum cohesion strength $\chi l \tau_{\text {dis }}^{u}$ provided by the distributed unions. If the cross-links provide a greater cohesion strength, a plane of weakness will appear along the distributed unions (failure mode 1), and if otherwise, a plane of weakness will appear along the cross-links (failure mode 2). Fig. 3.7 displays both planes of weakness, which form the following angles with the fibril axis direction (see Fig. 3.4 for the geometrical parameters):

$$
\tan \theta_{1}=\frac{D}{l-h+s} ; \quad \tan \theta_{2}=\frac{D}{h}
$$

We define now a critical length $l_{\text {cr }}$ as the minimum length of the TC molecule such that the maximum strength of the cross-links equals the maximum strength of the distributed unions:

$$
l_{\mathrm{cr}}=\frac{\eta \beta \tau_{\mathrm{dis}}^{u}}{\chi \tau_{\mathrm{dis}}^{u}}=\frac{\eta \beta}{\chi} .
$$

This parameter $l_{\text {cr }}$ gives us a criterion to identify which is the dominant failure mode in 


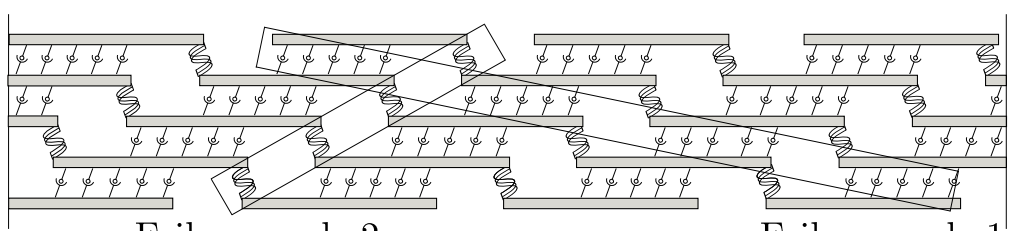

Failure mode 2

Failure mode 1

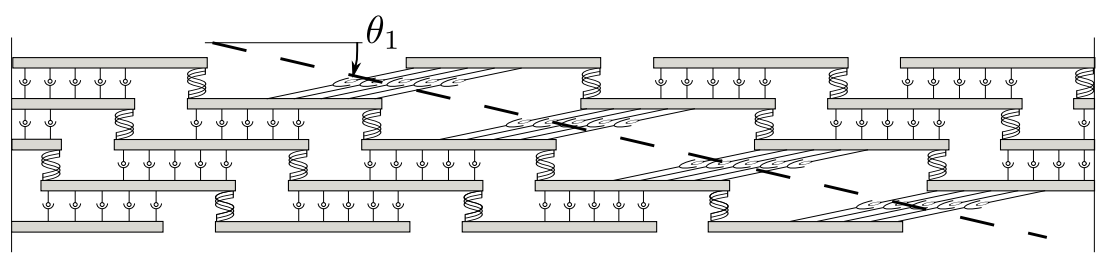

Failure mode 1

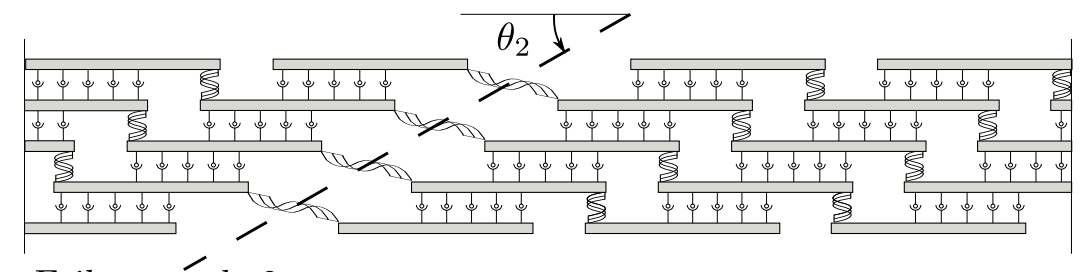

Failure móde 2

Figure 3.7: Fibril failure models

the fibril such that:

$$
\begin{aligned}
& \text { If } l<l_{\text {cr }} \longrightarrow \text { Failure mode 1, } \\
& \text { If } l>l_{\text {cr }} \longrightarrow \text { Failure mode } 2 \text {. }
\end{aligned}
$$

Fibril inelastic behaviour mesomechanical characterisation The onset and development of each of the previous failure modes affect the maximum tension of the fibril in elastic regime and the total amount of available dissipation energy. We describe now the mechanical characterisation for both failure modes and we derive afterwards the overall behaviour of the fibril, assuming that the length of the TC molecules inside the fibril follows a normal distribution.

Failure mode 1 is associated with the decohesion of the distributed unions between TC molecules. The maximum stress per unit area of the fibril is therefore:

$$
\sigma_{\text {fibril }}^{\mathrm{u}-\text { mode } 1}=\frac{\chi l \tau_{\text {dis }}^{u}}{D^{2}}
$$

where $D$ stands for the equilibrium distance between tropocollagen molecules, and the surface density of dissipated energy per unit area of the fibril is:

$$
\mathcal{G}_{\text {fibril }}^{\text {mode } 1}=\frac{1}{2} \tau_{\text {dis }}^{u}(\chi l)^{2} \frac{1}{D^{2}}
$$


Failure mode 2 is associated with the decohesion of the cross-links forces between the ends of TC molecules. In this case the maximum stress per unit area of the fibril is:

$$
\sigma_{\text {fibril }}^{\mathrm{u}-\text { mode } 2}=\frac{\eta \beta \tau_{\text {dis }}^{u}}{D^{2}}
$$

and the surface density of dissipated energy per unit area of the fibril is:

$$
\mathcal{G}_{\text {fibril }}^{\text {mode } 2}=\frac{1}{2} \beta \tau_{\text {dis }}^{u} \eta^{2} \frac{1}{D^{2}}
$$

The characteristic length $l_{\text {cr }}$ defined in equation (3.22) allows us to discriminate which failure mode will take place in the fibril. Assuming the lengths of the TC molecules inside a fibril have a normal distribution (characterised by a mean length $l_{m}$ and a variance $\sigma$, see Fig. 3.8), we can define the proportion of mode 1 failures inside a fibril as:

$$
D_{1}=\frac{1}{2}\left[1+\operatorname{erf}\left(\frac{l_{\mathrm{cr}}-l_{m}}{\sigma \sqrt{2}}\right)\right] .
$$

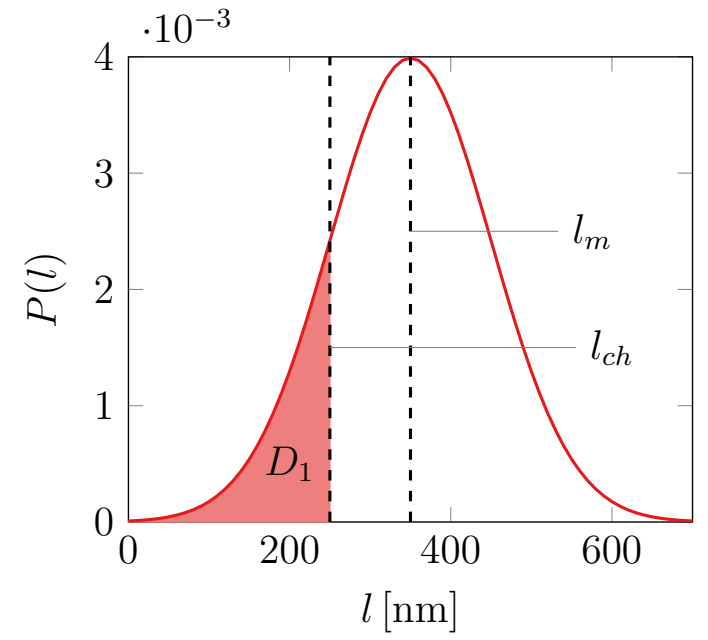

(a)

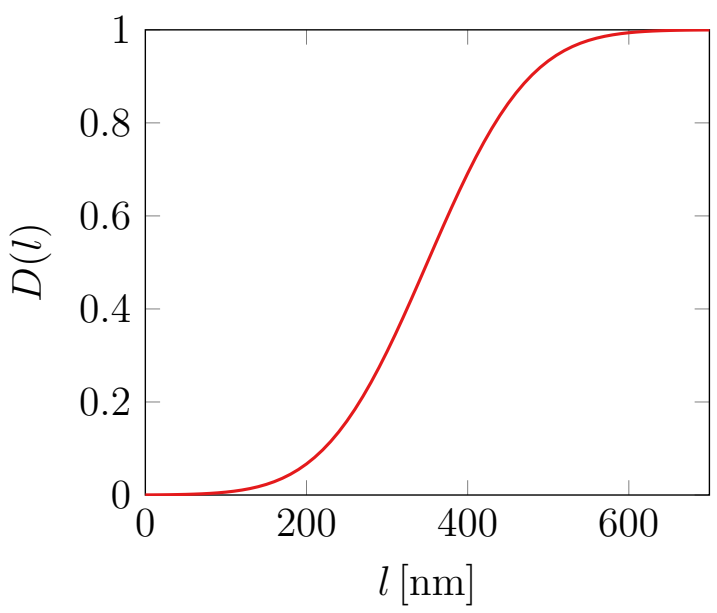

(b)

Figure 3.8: Probabilistic distribution of the TC molecular length. (a) Probability density function. (b) Cumulative distribution function.

Equations (3.25) to (3.29) give us finally the first two expressions that characterise the fibril mechanical behaviour in the mesoscopic scale:

$$
\begin{aligned}
& \sigma_{\text {fibril }}^{\mathrm{u}}=D_{1} \sigma_{\text {fibril }}^{\mathrm{u}-\text { mode } 1}+\left[1-D_{1}\right] \sigma_{\text {fibril }}^{\mathrm{u}-\text { mode } 2} \\
& \mathcal{G}_{\text {fibril }}=D_{1} \mathcal{G}_{\text {fibril }}^{\text {mode } 1}+\left[1-D_{1}\right] \mathcal{G}_{\text {fibril }}^{\text {mode } 2}
\end{aligned}
$$

where we have considered that the overall behaviour of the fibril is the weighted average of the behaviours of modes 1 and 2 . 
The stored elastic energy density per unit volume of the fibril at the onset of damage has two components: the stored elastic energy in the TC molecule and the stored elastic energy in the intermolecular adhesion unions (see Fig. 3.5). Both the TC molecule and the intermolecular adhesion unions undergo the same level of internal force (owing to the series arrangement):

$$
f_{\text {fibril }}^{u}=\sigma_{\text {fibril }}^{\mathrm{u}} D^{2}
$$

Assuming linear elastic behaviour for each component, and using the elastic stiffness $E_{\text {col }}$ and $K_{\text {dist }}$ for the collagen and the intermolecular adhesion unions, we can obtain the elastic energy stored in the TC molecule as (see Fig. 3.5):

$$
w_{\mathrm{TC} \text { molecule }}^{0}=\frac{1}{2} f_{\text {fibril }}^{u} \frac{f_{\text {fibril }}^{u}[1-\chi] l}{E_{\mathrm{col}} d^{2}}
$$

and the elastic energy stored in the intermolecular adhesion unions as:

$$
w_{\text {dist unions }}^{0}=\frac{1}{2} f_{\text {fibril }}^{u} \frac{f_{\text {fibril }}^{u}}{K_{\text {dist }}}
$$

Expressions (3.33)-(3.34) give us finally an elastic energy density per unit volume of the fibril:

$$
\Psi_{\text {fibril }}^{0}=\frac{1}{l D^{2}}\left[w_{\mathrm{TC} \text { molecule }}^{0}+w_{\text {dist unions }}^{0}\right]
$$

The values derived in equations (3.30), (3.31) and (3.35) allows us to characterise the mechanical behaviour of the fibril in the mesoscopic scale using the mechanical properties of its basic components.

\subsubsection{Fibre macromechanical characterisation}

We assume fibrils are aligned with the fibre axis and fibres of the same family share a common mean direction inside the tissue. We also consider the fibre and the tissue as homogenised continua (the fibre as a fibril reinforced composite and the tissue as a fibre reinforced composite). This approach allows us to define the homogenised properties of each composite as functions of the mechanical properties of the constituents and its volume fractions.

In this section we firstly obtain the mesoscopic properties of the fibre. To do so, we define the volume fractions of the fibrils and the ground matrix in terms of their relative thicknesses inside the fibre. Afterwards, we obtain the macroscopic properties of the fibre in terms of the volume fraction of the fibre inside the tissue. Finally we calculate, for each family of fibres, the material parameters of the continuum damage model introduced in Section 3.1 (see parameters in equations (2.39) and (3.17)). We impose that the inelastic 
properties of this model, i.e. the uniaxial stretch and stress elastic thresholds, the stored elastic energy that defines the initial elastic domain and the surface density of dissipated energy, are given by the macroscopic values derived in the upscaling homogenisation process.

Fibre mesomechanical model Fibres can be considered as a bundle of closely packed thin fibrils linked by very thin proteoglycan filaments (GAGs). Proteoglycans determine the distance of two neighbouring fibrils to fasten themselves into a bundle [91]. In this work we consider a fibre two-dimensional model characterised only by the fibril diameter $B_{0}$ and the interfibrillar distance $B_{1}$ (see Fig. 3.9a). We assume also there is a compatibility of strains for both the fibril and the proteoglycan-rich ground matrix phases. Following these assumptions the mechanical properties of the fibre at mesoscale level are:

$$
\begin{aligned}
\sigma_{\text {fibre }}^{\mathrm{u}} & =\frac{B_{0}}{B_{0}+B_{1}} \sigma_{\text {fibril }}^{\mathrm{u}}+\frac{B_{1}}{B_{0}+B_{1}} \sigma_{\mathrm{GAG}}^{\mathrm{u}} \approx \frac{B_{0}}{B_{0}+B_{1}} \sigma_{\text {fibril }}^{\mathrm{u}}, \\
\Psi_{\text {fibre }}^{0} & =\frac{B_{0}}{B_{0}+B_{1}} \Psi_{\text {fibril }}^{0}+\frac{B_{1}}{B_{0}+B_{1}} \Psi_{\mathrm{GAG}}^{0} \approx \frac{B_{0}}{B_{0}+B_{1}} \Psi_{\text {fibril }}^{0}, \\
\mathcal{G}_{\text {fibre }} & =\frac{B_{0}}{B_{0}+B_{1}} \mathcal{G}_{\text {fibril }}+\frac{B_{0}}{B_{0}+B_{1}} \mathcal{G}_{\mathrm{GAG}},
\end{aligned}
$$

where we have assumed that (a) the proteoglycan-rich matrix and the fibril have a parallel arrangement (see Figure 3.9b) and (b) the elastic stiffness of the GAGs molecules is so low that we they don't contribute to the yield stress and the stored elastic energy of the fibre. However, the ductile rupture of the GAGs molecules do provide surface density of dissipation energy.

Table 3.4 summarises the mechanical and geometrical properties of the fibre two-dimensional model used in equations (3.36)-(3.38).

\begin{tabular}{lcc}
\hline Component & Symbol & Value \\
\hline $\begin{array}{l}\text { GAG's molecules surface } \\
\text { density of dissipation energy }\end{array}$ & $\mathcal{G}_{\mathrm{GAG}}$ & $10^{3} \mathrm{pN} / \AA$ \\
$\begin{array}{l}\text { Fibril width } \\
B_{0}\end{array}$ & $180 \mathrm{~nm}$ \\
$\begin{array}{l}\text { Proteoglycan-rich } \\
\text { matrix width }\end{array}$ & $B_{1}$ & $100 \mathrm{~nm}$ \\
$\begin{array}{l}\text { Fibre volume fraction } \\
\text { in the tissue }\end{array}$ & $\nu^{f}$ & $0.22^{(\mathrm{a})}$ \\
\hline $\begin{array}{l}{ }^{(a)} \text { See Fig. 4 in [92] } \\
\quad\end{array}$ & \\
&
\end{tabular}


(a)

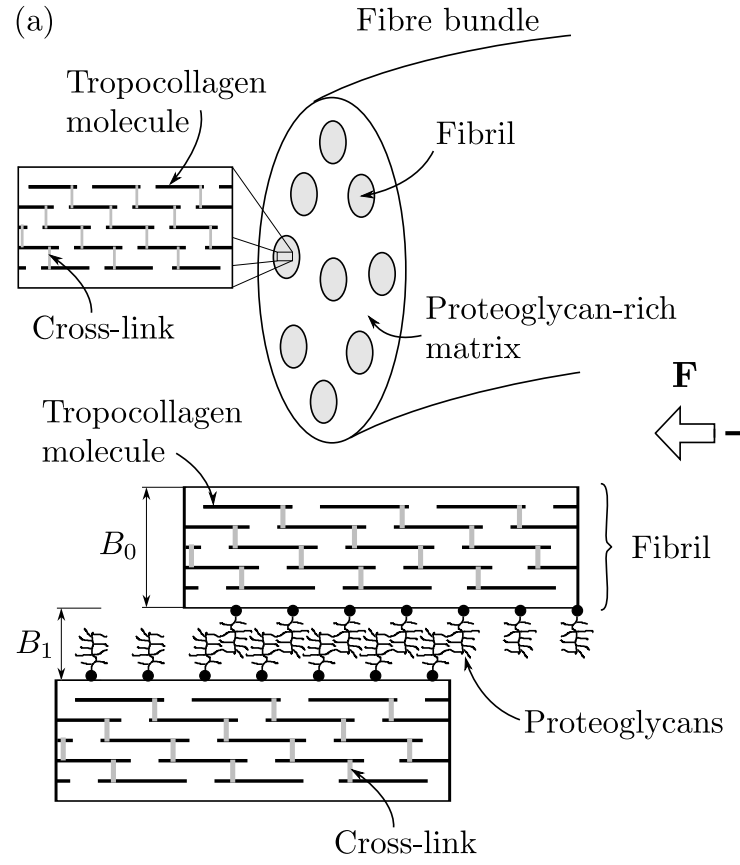

(b)

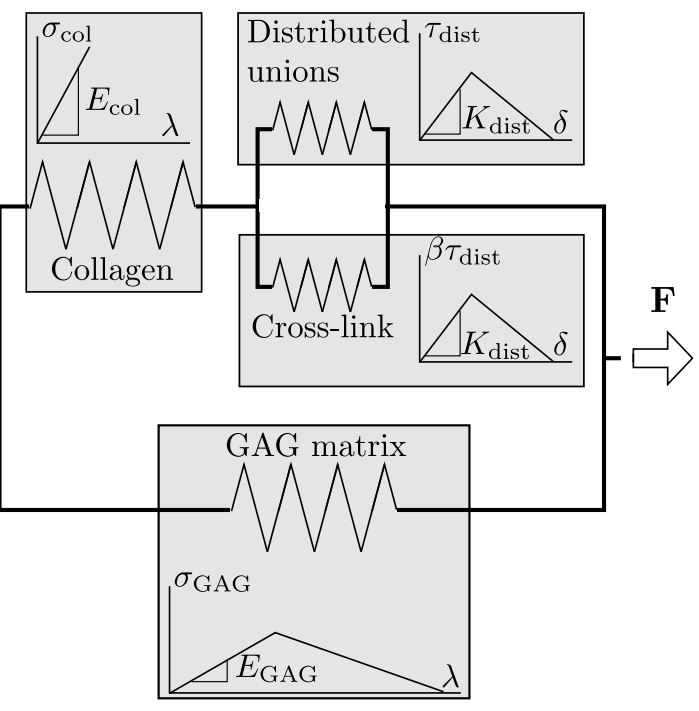

Figure 3.9: 2-D fibre model

Fibre macroscopic elastic energy density function At macroscopic scale we have modelled soft tissues as composites formed by an incompressible ground matrix and one or several families of fibres. Fibres are represented in the composite by a volumetric fraction $\nu^{f}$ and therefore the stresses and energy densities of equations (3.36)-(3.38) must be homogenised in order to obtain the macroscopic values of the fibre at tissue level. Macroscopic expressions of the mechanical properties of the fibres are thus obtained as:

$$
\begin{aligned}
\sigma_{\text {fibre }}^{\mathrm{u}-\text { tissue }} & =\nu^{f} \sigma_{\text {fibre }}^{\mathrm{u}} \\
\Psi_{\text {fibre }}^{0-\text { tissue }} & =\nu^{f} \Psi_{\text {fibre }}^{0} \\
\mathcal{G}_{\text {fibre }}^{\text {tissue }} & =\nu^{f} \mathcal{G}_{\text {fibre }}
\end{aligned}
$$

where $\nu^{f}$ is the fibre volume fraction in the tissue. Mechanical properties of the incompressible ground matrix depend on the properties of the GAGs molecules. It is commonly accepted that this material can be modelled at the macroscopic scale with a neo-Hookean law [93].

The values of $\sigma_{\text {fibre }}^{\mathrm{u} \text {-tissue }}$ and $\Psi_{\text {fibre }}^{0-\text { tissue }}$ given in equations (3.40)-(3.41), along with the uniaxial elastic stretch threshold $\lambda_{e}$ given in equation (3.20), allow us to fully characterise the macroscopic constitutive model for the families of fibres. To achieve that we impose, in the uniaxial traction test and at the onset of damage, that the yield stress and the stored elastic energy density given by the constitutive model match the values of equations (3.40)-(3.41) (see Fig. 3.10). We consider henceforth in this chapter there is no fibre dispersion around 
the fibre's mean direction.

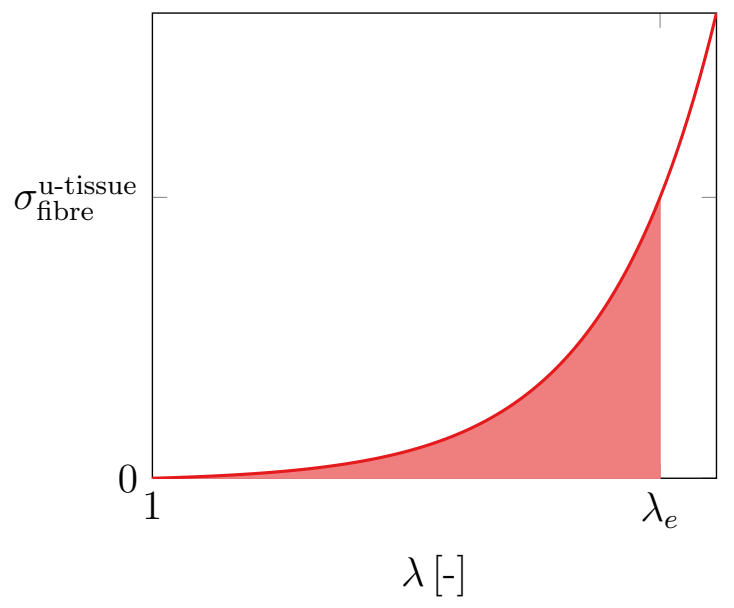

Figure 3.10: Uniaxial fibre model

The fibre effective strain energy function defined in equation (2.39), particularised for the uniaxial traction test when the fibre is aligned with the direction of stretching is:

$$
\Psi^{\mathrm{fibre}}=\frac{k_{1}}{2 k_{2}}\left[\exp \left(k_{2}\left(\lambda^{2}-1\right)^{2}\right)-1\right]
$$

where $k_{1}$ and $k_{2}$ are two material parameters to be determined and $\lambda$ is the stretch in the fibre axial direction. The fibre uniaxial stress is therefore:

$$
\sigma^{\text {fibre }}=\lambda \frac{\partial \Psi^{\text {fibre }}}{\partial \lambda}=2 k_{1}\left[\lambda^{4}-\lambda^{2}\right] \exp \left(k_{2}\left[\lambda^{2}-1\right]^{2}\right)
$$

Equations (3.43) and (3.44) allow us to finally obtain $k_{1}$ and $k_{2}$ as the solution of the following system of nonlinear equations:

$$
\begin{aligned}
\sigma^{\text {fibre }}\left(\lambda_{e}\right) & =2 k_{1}\left[\lambda_{e}^{4}-\lambda_{e}^{2}\right] \exp \left(k_{2}\left[\lambda_{e}^{2}-1\right]^{2}\right) \\
& =\sigma_{\text {fibre }}^{\text {u-tissue }} \\
\Psi^{\text {fibre }}\left(\lambda_{e}\right) & =\frac{k_{1}}{2 k_{2}}\left[\exp \left(k_{2}\left(\lambda_{e}^{2}-1\right)^{2}\right)-1\right] \\
& =\Psi_{\text {fibre }}^{0-\text { tissue }}
\end{aligned}
$$

where the values of $\sigma_{\text {fibre }}^{\mathrm{u} \text {-tissue }}$ and $\Psi_{\text {fibre }}^{0-\text { tissue }}$ are given in equations (3.40) and (3.41). The nonlinear equation system (3.45)-(3.46) has been numerically solved by using the optimize package of scipy library [94]. 


\subsection{Numerical examples}

In this section we have performed mechanical analysis at fibril, fibre and tissue levels. Our aim is to study the behaviour of the model at each of the hierarchical scales we have considered. Unless otherwise specified, the values of the mesoscopic parameters used to characterise the tissue are given in Tables 3.2, 3.3 and 3.4.

\subsubsection{Fibril behaviour analysis}

Firstly, we analyse how the cohesive force of the cross-links (defined as a function of parameter $\beta$, see Table 3.3) affects the yield strength of the fibril. The influence of $\beta$ parameter appears only when the material failure is associated with the breakage of cross-links (failure mode 2). In Fig. 3.11a we plot the stress level $\sigma_{\text {fibril }}^{\mathrm{u}}$ of the fibril at the onset of damage, see equation (3.30), as a function of parameter $\beta$. We see an increase in the yield strength with increasing the bearing capacity of the cross-links, until the strength of the cross-links is so large that failure occurs along the distributed unions in the overlapped length (failure mode 1). In Fig. 3.11a we compare our results with those presented in [18], where molecular dynamics (MD) simulations were made using a short chain of collagen molecules. Further MD simulations were performed in [24] increasing the size of the fibril until it behaved as a representative volume element. Results presented in [24] show a subtle increase in the yield strength (see Fig. 4 in [24]) if we compare with those presented previously in [18].

We study also how the length of the TC molecule affects the yield strength of the fibril when, in this case, material failure is associated with the breakage of the distributed unions along the overlapped length (failure mode 1). In Fig. 3.11b we see that there is an increase of the yield strength when we increase the length of the TC molecule, until it reaches a threshold value at which the failure occurs through the cross-links (failure mode 2).

Experimental tests of individual fibrils can be found in [95] and [96]. In these studies, the first deviation from linearity occurred at a yield strength of $0.22 \pm 0.14 \mathrm{GPa}$ (nominal stress). Our model predicts a stronger response of fibrils in case cross-links are present. In the authors' opinion, more research should be made to characterise the mechanical behaviour of the constituent elements of the fibril, in particular the mechanical interaction between two TC molecules along their overlapped length. Following [23], we have considered in this work an ultimate strength of the distributed unions of $\tau_{\text {dist }}^{u}=5.55 \mathrm{pN} / \AA$, which is also used to calculate the ultimate strength of the cross-links as $\tau_{\mathrm{c}-1}^{u}=\beta \tau_{\text {dist }}^{u}$. In order to better approximate the experimental results presented in [95] and [96], a smaller value of parameter $\tau_{\text {dist }}^{u}$ should be used. 


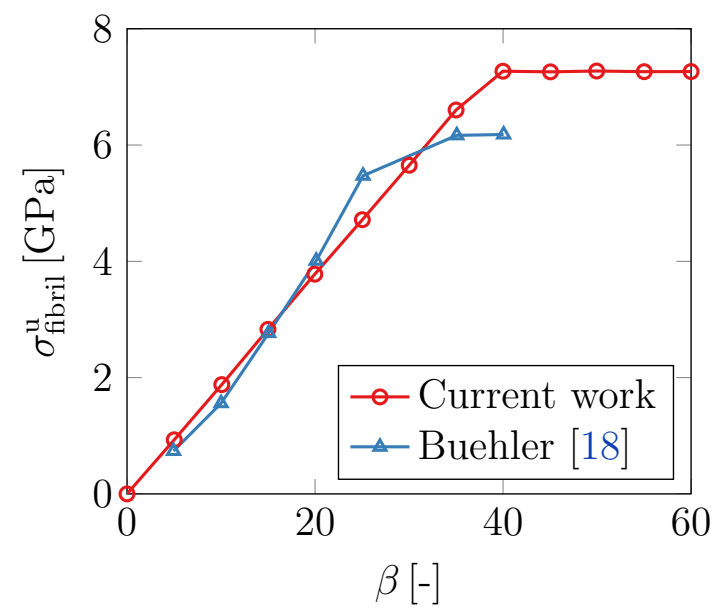

(a)

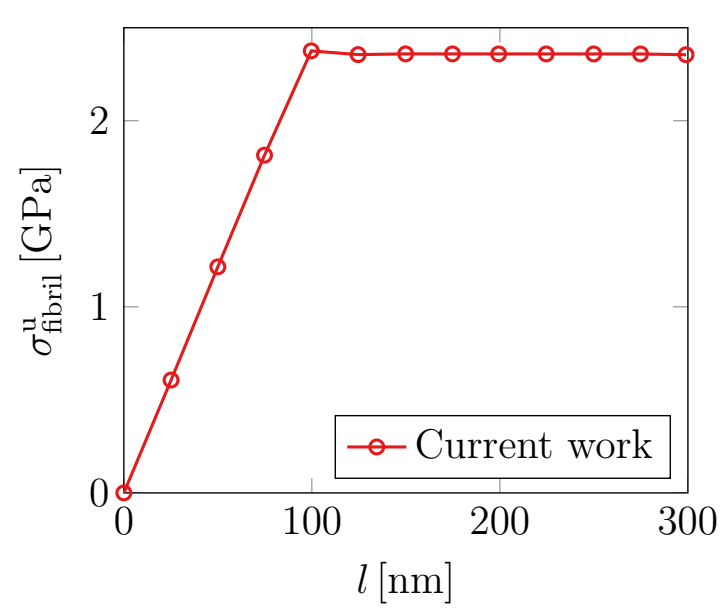

(b)

Figure 3.11: (a) Fibril yield strength as a function of cross-link $\beta$ parameter. (b) Fibril yield strength as a function of the length of the TC molecule.

\subsubsection{Fibre behaviour analysis}

We use the homogeneous simple tensile test to analyse the behaviour of the fibres. The test specimen is a parallelepiped with dimensions $a=2.81 \mathrm{~mm}, b=0.32 \mathrm{~mm}$ and $h=12.5$ $\mathrm{mm}$ (see Fig. 3.12a). The matrix of the composite is assumed to have null strength and the fibres are oriented in the direction of stretching. In this section we perform a sensitivity analysis of the following parameters: the cross-link strength (Fig. 3.12b), the TC molecular length (Fig. 3.12c) and the waviness parameter (Fig. 3.12d). These stress-stretch curves present tension-stiffening in the elastic domain associated with the fibril recruitment along the stretching direction. The onset of inelastic processes in the fibre is associated with a discontinuity (a kink) in the slope of the curves stress-stretch. After that point, the regularised damage model of Section 3.1 requires the total dissipated energy to be given by the material parameter surface density of dissipated energy, defined in equation 3.38. Therefore, the shape of the softening branch of the curves depends on the size (the length $h$ in Fig. 3.12a) of the domain, i.e., the larger the length $h$ the brittler the inelastic behaviour. We firstly investigate the influence of the cross-link strength ( $\beta$ parameter) on the fibre stress-stretch response. In Fig. 3.12b we compare the stress-stretch behaviour for different values of $\beta$. As we can see the yield strength and the brittleness of the model increase with increasing $\beta$. We find a similar behaviour when we increase the length of the TC molecule. In Fig. 3.12c we show the stress-stretch responses for different values of $l_{\mathrm{m}}$. When we increase the value of the TC molecular length, we obtain an increasing value of the yield strength and a slight increase of the brittle behaviour (see the different vertical scales in Figs. 3.12b and 3.12c). We finally study the fibre behaviour when the fibril waviness is modified. In Fig. 3.12d we plot the stress-stretch curves when the waviness parameter $r$ is 


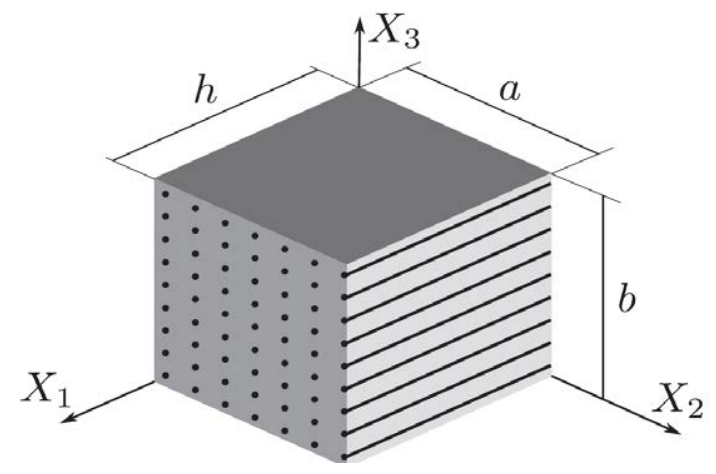

(a)

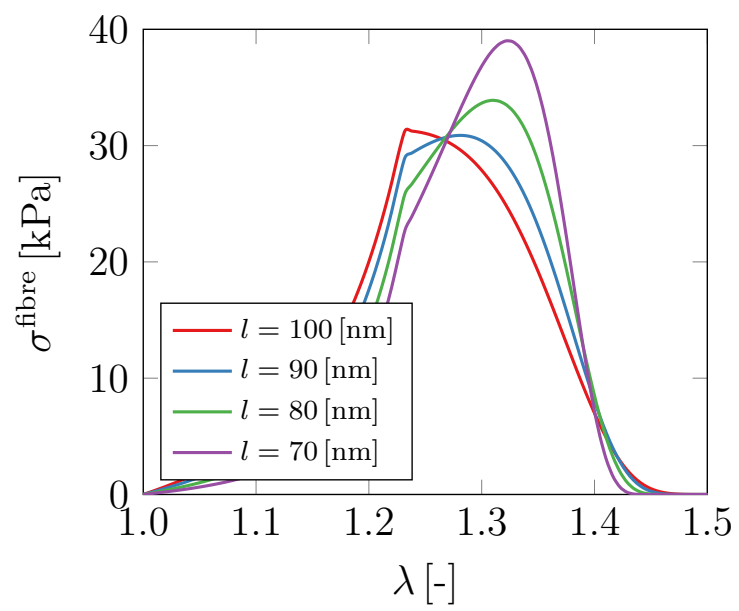

(c)

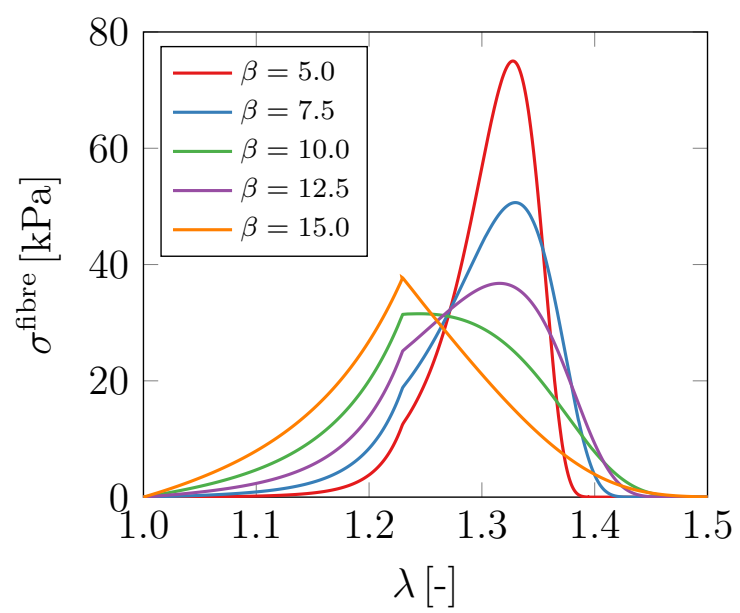

(b)

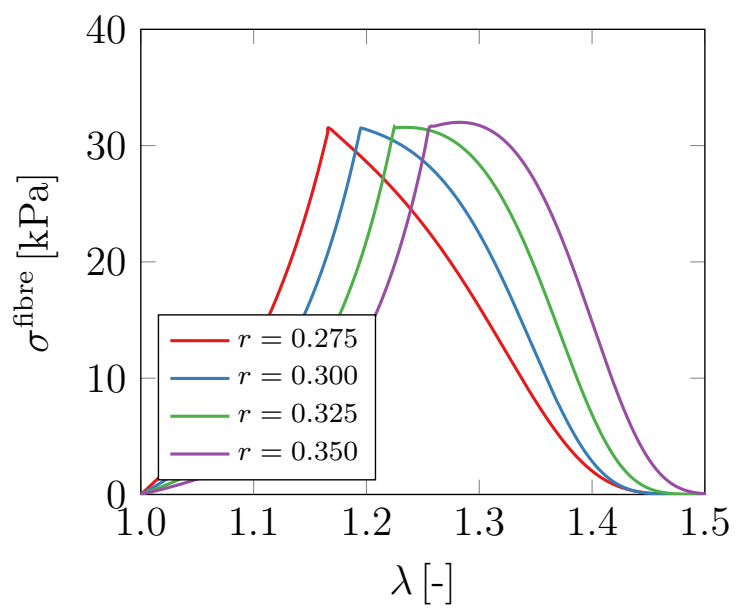

(d)

Figure 3.12: Fibre behaviour analysis in a uniaxial test. (a) Single element mesh. (b) $l=300[\mathrm{~nm}]$, $r=0.33[-]$. (c) $\beta=12.5[-], r=0.33[-]$. (d) $l=300[\mathrm{~nm}], \beta=12.5[-]$.

increased. We appreciate that when we increase the value of $r$ the elastic stretch threshold is increased and a small decrement of the brittleness takes place.

\subsubsection{Soft tissue behaviour analysis}

Mesoscopic values of Tables 3.2, 3.3 and 3.4 give us the derived macroscopic fibre material parameters at tissue level summarised in Table 3.5.

Several works have been published with numerical fitting of material parameters $\left\{k_{1}, k_{2}\right\}$ to the elastic physiological response of fibred soft tissues (see [97], [98] or [99] among others). In [98], range values of $\left\{k_{1}=[240,940] \mathrm{kPa}, k_{2}=[5.790,34.79]\right\}$ are given for healthy thoracic aorta and $\left\{k_{1}=[2580,10500] \mathrm{kPa}, k_{2}=[0.00,32.4]\right\}$ for healthy abdominal aorta. However, these values are not exactly comparable with those of Table 3.5 because these works have used an extended version of equation 2.39 taking into account the dispersion in 


\begin{tabular}{lccc}
\hline Symbol & Value & Units & Equation \\
\hline$k_{1}$ & 1704.3 & {$\left[\mathrm{kN} / \mathrm{m}^{2}\right]$} & $(3.45)-(3.46)$ \\
$k_{2}$ & 3.585 & {$[-]$} & $(3.45)-(3.46)$ \\
$\sigma_{u}$ & 6.81 & {$\left[\mathrm{MN} / \mathrm{m}^{2}\right]$} & $(3.40)$ \\
$\mathcal{G}_{f}$ & 1.38 & {$[\mathrm{~N} / \mathrm{m}]$} & $(3.42)$ \\
\hline
\end{tabular}

Table 3.5: Macroscopic fibre material parameters.

the collagen fibre directions (see Gasser et al. [100] for more details).

Experimental data about failure properties of soft tissues can be found in literature although these values present some variability. Material parameters associated with fracture of soft tissues are associated to different types of tests: tensile, inflation, tearing, splitting, and peeling tests (see [101] and references therein). If we restrain ourselves to the aortic tissue, we find values of the axial strength from $1.21 \pm 0.33 \mathrm{MPa}$ in human abdominal aorta [102] to $1.95 \pm 0.60 \mathrm{MPa}$ in human descending thoracic aorta [103]. The mesoscopic parameters chosen in our model lead to a value (6.81 MPa) larger than the experimental axial strength found in aortic tissues. One possible cause of this discrepancy could be our overestimation of the tensile strength of the fibril that has already been discussed in Section 3.3.1. There is also uncertainty in the value of the dissipated energy of collagenous fibres in soft tissues in the extension test. We find that the value of dissipated energy in the aortic tissue in the splitting test ranges from $1.7 \mathrm{~N} / \mathrm{m}$ in human thoracic and abdominal aorta [104] to $159 \pm 9$ $\mathrm{N} / \mathrm{m}$ in porcine descending thoracic aorta [105]. When we compare these values with the one presented in Table 3.5 we must bear in mind that the failure modes considered are different (extension vs. splitting).

In this section we study the deformation of a square plate with a hole (see Fig. 4.4(a)) when it is stretched in a tensile test. An increasing displacement is imposed along the $X_{1}$ direction at sections $\mathrm{A}$ and $\mathrm{A}$ ' while the remaining displacement degrees of freedom are set to zero. The study has mirror symmetry so we discretise only one eighth of the model (the domain placed in the first octant). We use three geometrical discretisations, called Mesh1, Mesh2 and Mesh3, with different characteristic element lengths, see Table 3.6 and Figs. 3.14(a)-3.14(c)).

\begin{tabular}{lccc}
\hline Name & Elements & Type & $\begin{array}{c}\text { Characteristic } \\
\text { length } h\end{array}$ \\
\hline Mesh1 & 64 & 8-node brick & $25.0 \mathrm{~mm}$ \\
Mesh2 & 256 & 8-node brick & $12.5 \mathrm{~mm}$ \\
Mesh3 & 576 & 8-node brick & $8.33 \mathrm{~mm}$ \\
\hline
\end{tabular}

Table 3.6: Mesh geometric properties.

Only one family of fibres is considered. This family is oriented in the $X_{1}$ direction, so 
its orientation vector at the reference configuration is $\mathbf{a}_{0}=(1,0,0)$. We characterise the mechanical behaviour of the fibres with the material parameters summarised in Table 3.5. These fibre parameters were obtained from mesoscopic values in Tables 3.2, 3.3 and 3.4. Ground matrix behaviour is reproduced by using a fully elastic neo-Hookean model with shear modulus $c=5 \mathrm{MN} / \mathrm{m}^{2}$.

In this displacement-controlled tension test we increase monotonically the prescribed displacement from zero up to a maximum value of $56.3 \mathrm{~mm}$. In Fig. 3.14a we show the forcestretch response of the model. The two solid black curves represent the fully elastic response of the composite (upper) and the isotropic elastic neo-Hookean matrix (lower). Discontinuous lines represent the force-stretch response for the discretisations Mesh1, Mesh2 and Mesh3. We can identify the onset of damage around the stretch value $\lambda=1.15$, when the equilibrium curves of the damaged composite separate from the fully elastic behaviour. The equilibrium curves obtained with the three geometrical discretisations are quite similar due to the softening regularisation explained in Section 2.1.4.

Although this model enforces the mesh-objectivity of the structural response two difficulties emerge: the definition of the correct characteristic length for each finite element used in equation (3.18) and the influence of the mesh bias on the numerical results. The first problem is solved by defining an approximate characteristic length as the size of the element in the stretching direction (see Table 3.6). In Fig. 3.14b-d the contours of the damage function are plotted for the three geometrical discretisations. As we can see, damage is more pronounced around the tip of the hole where the resistant section is smaller and where we find the highest values of the stresses. The domain completely damaged in the three numerical simulations is almost the same.

\subsection{Summary}

The aim of the model presented in this section was to establish a relationship between the mesoscopic structural mechanisms that rule inelastic processes in fibrous soft tissues and their macroscopic material parameters. A phenomenological continuous damage model with regularised softening was presented along with an interpretation of the damage processes that take place at fibril scale in soft tissues. This interpretation allows us to derive a relationship between the mechanical and geometrical properties of the fibril constituents (tropocollagen molecules and the cohesive forces between them) and the material parameters of the damage model. We have obtained meso-structurally based definitions for the elastic parameters defining the effective strain energy function, and for the inelastic parameters defining the ingredients of the damage model (yield strength and surface density of total dissipated energy).

We have interpreted soft tissues as fibre reinforced composites and fibres as fibril reinforced 


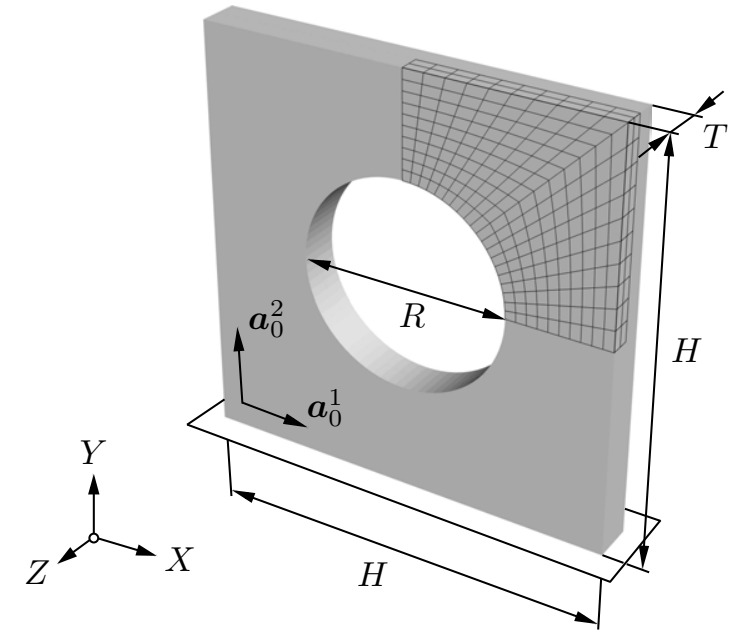

(a)

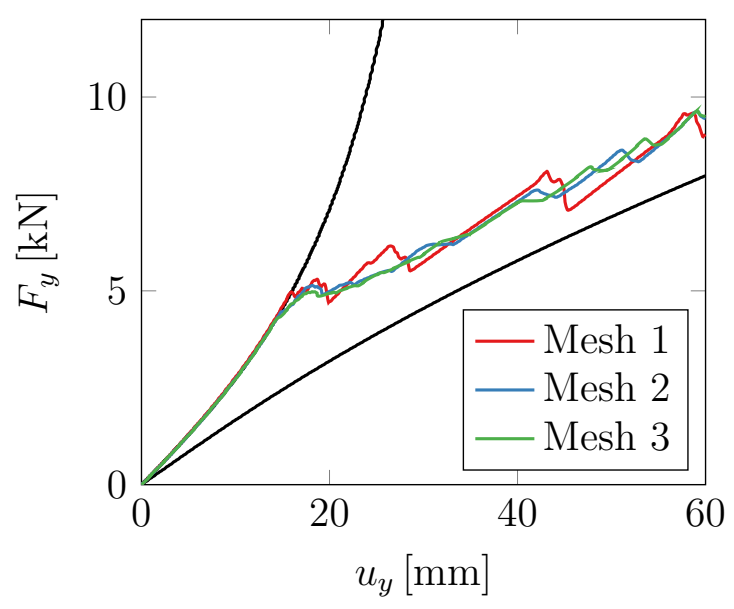

(b)

Figure 3.13: Plate with a hole. (a) Dimensions $(R=150[\mathrm{~mm}], H=200[\mathrm{~mm}], T=20[\mathrm{~mm}])$, orthotropic fibre orientations $\boldsymbol{a}_{0}^{1}, \boldsymbol{a}_{0}^{2}$ and discretisation (1/8 of the full geometry). (b) Forcedisplacement response and investigation of mesh-sensitivity. The solid black curves represent the elastic response of the isotropic neo-Hookean matrix (lower curve) and the overall elastic response of the fibre-reinforced material (upper curve).

composites. A Hodge-Petruska bidimensional model has been used to describe the fibrils as staggered arrays of tropocollagen molecules. After a mechanical characterisation of each of the fibril components, two fibril failures modes have been defined related with two planes of weakness. These planes of weakness are associated with either the decohesion of the distributed cohesive forces between the overlapped length of the TC molecules, or the decohesion of the concentrated cohesive forces at the end of the TC molecules. The prevailing mode of failure is a function of the TC molecular length, which allows us to characterise the fibril mechanical properties in terms of the mechanical properties of its constituents and the probability distribution of the TC molecular length. Finally, we obtain the macroscopic material properties of the fibres using an homogenisation process. We have presented a phenomenological damage model for fibrous materials with regularised softening. Damage processes depend only on the isochoric deformation and ground matrix and fibres damage independently. We have defined a strain energy function with dilatational and isochoric contributions. The dilatational contribution has been decomposed into an isotropic part associated with the ground matrix and an anisotropic part associated with the families of fibres. We have used the principles of the smeared crack approach to avoid the mesh size dependence of the structural response in softening. We have defined a regularised softening modulus in terms of a surface density of dissipated energy and a characteristic size of the finite element.

This phenomenological damage model has been implemented as a subroutine inside the finite element code F.E.A.P. Taylor [106]. The process of obtaining the macroscopic ma- 


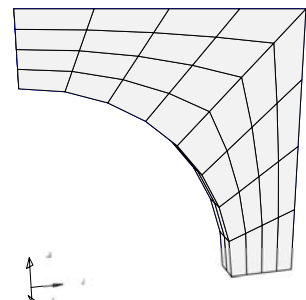

(a)

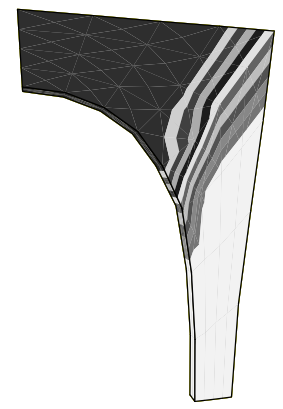

(d)

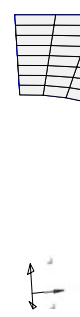

(b)

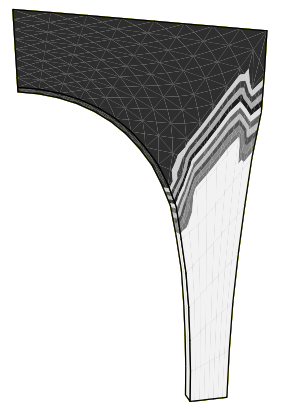

(e)

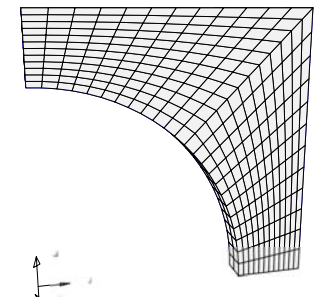

(c)

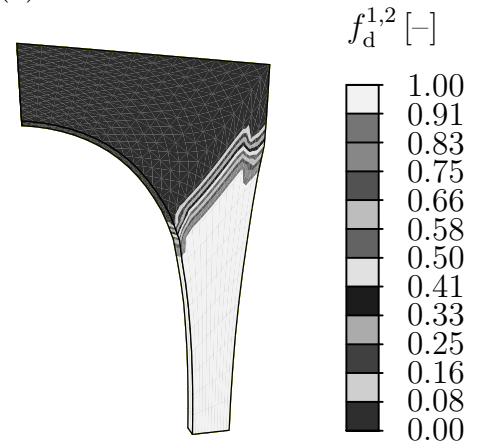

(f)

Figure 3.14: Undeformed mesh. (a) Mesh1. (b) Mesh2. (c) Mesh3. Contour plot of damage function $f_{\mathrm{d}}^{1,2}$. (d) Mesh1. (e) Mesh2. (f) Mesh3.

terial parameters from the mesoscopic fibril model was made using the environment SciPy Jones et al. [107].

The present approach to obtain the material parameters of the phenomenological damage model in terms of the fibril mesostructure has the advantage of its simplicity in use. The complexity of the model is reduced to an initial study to obtain the derived material parameters of the damage model without any further modification in the finite element code. We are able to incorporate the properties of the fibrous tissue structure without using the computationally more expensive multiscale models. It allows us to study the influence of the geometrical and mechanical properties of the fibril constituents in a nonexpensive, straightforward way.

However, this work presents also some limitations. In the bottom-up approach we have followed, we have used the mechanical definitions (stiffness, yield strength and density per unit area of dissipated energy) of the fundamental components of soft tissues at fibril scale. These characterisations, along with the volume fractions of each component at fibre and tissue level, present a high degree of uncertainty, especially the definition of the densities per unit area of dissipated energy. Some of the numerical values considered in Tables 3.2, 3.3 and 3.4 are based in molecular dynamics simulations, some are based in experimental tests or observations, and the others are just a choice of the authors because no experimental value has been found. A more precise geometric and mechanical characterisation is required for each of the fundamental constituents of the specific soft tissue we want to analyse.

Finally we would like to mention some limitations of the model related with the simplifying 
hypothesis we have considered. Firstly, we have assumed the same elastic stretch threshold for both the fibril and the fibre. This is the same as considering that fibril and fibre undergo the same strain at both length scale levels. We have also considered at fibril scale that all TC molecules deform uniformly. Intuitively we might think that in the Hodge-Petruska model there will be a stress concentration at the end of the TC molecules. Besides, we have neglected the possibility of a failure of the tropocollagen molecule when we have defined the fibril failure modes. Finally, and associated also with the fibril failure modes, we have used a distribution function of probability that can theoretically provide non-zero probability for negative values of the TC molecular length (when a standard deviation too large compared with the mean value is used, although this is a non-physiological situation). 


\section{Non-local gradient-enhanced continuum damage model for fibred biological tissues

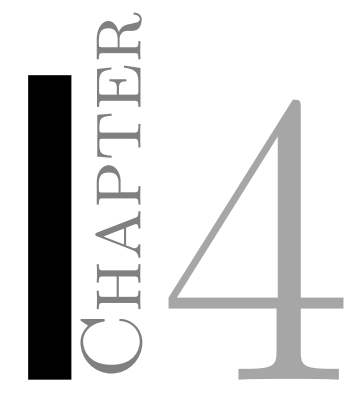

In this chapter we present a non-local gradient-enhanced continuum damage formulation at large deformations. At the material point, the elastic constitutive behavior is defined by a hyperelastic functional made of a volumetric and an isochoric contribution. For the volumetric contribution, a quadratic form that serves to enforce the incompressibility constraint $J-1=0$ is used. The isochoric contribution is further subdivided into three contributions associated to three different phases $i=0,1,2$. Phase 0 is represented by an incompressible neo-Hookean material; whereas phases 1 and 2 are represented by an exponential format that accounts for the stretching along two preferred anisotropy directions, i.e. two fibre families. Furthermore, a $1-d^{i}$-type damage function, is introduced to reproduce the loss of stiffness in each phase $i$. Following the ideas discussed on Dimitrijević and Hackl [55], the model is built around the enhancement of the local free energy function by means of a term that contains the referential gradient of the non-local damage variable $\phi^{i}$. The inclusion of this term ensures an implicit regularisation of the finite element implementation, ensuring mesh-objective results of the simulations. Furthermore, a penalisation term is incorporated to enforce the equivalence of the local and non-local damage variables. The Euler-Lagrange equations that govern the boundary value problem are solved in their weak form by means of the minimisation of the total potential energy. The weak form, in turn, serves as the basis for the finite element implementation of the non-local gradientenhanced continuum damage model. To this end we develop an 8-noded Q1P0 hexahedral element following a multivariational approach, which ensures the fulfillment of the quasiincompressibility condition. This element is implemented in Abaqus by means of the user subroutine UEL. Two boundary value problems are studied: a homogeneous deformation test, and an anisotropic plate with a hole. These experiments serve to illustrate the main capabilities of the proposed model, as well as to highlight its applicability to the modelling of soft tissues. 


\subsection{Gradient enhancement of a continuum damage formulation}

As a starting point, we assume the existence of a local free energy function $\Psi_{\text {loc }}$ for a fibre-reinforced material defined per unit reference volume

$$
\Psi_{\mathrm{loc}}\left(\boldsymbol{F}, \boldsymbol{H}^{i}, \kappa^{i}\right)=\Psi_{\mathrm{loc}}^{\mathrm{vol}}(J)+f_{\mathrm{d}}^{0}\left(\kappa^{0}\right) \Psi_{\mathrm{loc}}^{0}(\overline{\boldsymbol{F}})+\sum_{i=1,2} f_{\mathrm{d}}^{i}\left(\kappa^{i}\right) \Psi_{\mathrm{loc}}^{i}\left(\overline{\boldsymbol{F}}, \boldsymbol{H}^{i}\right)
$$

that accounts for an anisotropic non-linear elastic material response under the influence of scalar damage. $\Psi_{\text {loc }}^{\mathrm{vol}}$ represents the free energy term associated to the volumetric deformation, while the isochoric response is composed of the contributions of three hyperelastic functionals $\Psi_{\mathrm{loc}}^{i}$ for different phases $(i=0,1,2) . \quad \Psi_{\mathrm{loc}}^{0}$ is an isotropic contribution, and $\Psi_{\text {loc }}^{1,2}$ is the anisotropic contribution due to two fibre-reinforcements. In equation (4.1), $\kappa^{i} \in[0, \infty)$ is a scalar internal damage variable characterising a material stiffness loss in the $i$-th phase, while $f_{\mathrm{d}}^{i}\left(\kappa^{i}\right)=1-d^{i}$ represents an appropriate damage function that is at least twice differentiable and satisfies

$$
f_{\mathrm{d}}^{i}\left(\kappa^{i}\right): \mathbb{R}^{+} \rightarrow(0,1] \mid\left\{f_{\mathrm{d}}^{i}(0)=1, \lim _{\kappa^{i} \rightarrow \infty} f_{\mathrm{d}}^{i}\left(\kappa^{i}\right) \rightarrow 0\right\} .
$$

These conditions assure purely elastic behaviour of the undamaged material and complete loss of material stiffness in the limiting case $\kappa^{i} \rightarrow \infty$, cf. Dimitrijević and Hackl [55].

Following the concept of Dimitrijević and Hackl [55], a gradient-enhanced non-local free energy function $\Psi_{\text {nloc }}$ is introduced as

$$
\Psi_{\text {nloc }}\left(\boldsymbol{F}, \kappa^{i}, \phi^{i}, \nabla_{\boldsymbol{X}} \phi^{i}\right)=\sum_{i=0,1,2} \Psi_{\text {nloc }}^{i}=\sum_{i=0,1,2}\left[\Psi_{\text {grd }}^{i}\left(\boldsymbol{F}, \nabla_{\boldsymbol{X}} \phi^{i}\right)+\Psi_{\text {plty }}^{i}\left(\kappa^{i}, \phi^{i}\right)\right]
$$

where each $\Psi_{\text {grd }}^{i}$ contains the referential gradient of the non-local damage field variable $\phi^{i}$, and where each $\Psi_{\text {plty }}^{i}$ is a penalisation term that enforces an equivalence-constraint between the non-local damage variable $\phi^{i}$ and the local damage variable $\kappa^{i}$. Consequently, we obtain an enhanced free energy function as

$$
\Psi_{\text {int }}\left(\boldsymbol{F}, \boldsymbol{H}^{i}, \kappa^{i}, \phi^{i}, \nabla_{\boldsymbol{X}} \phi^{i}\right)=\Psi_{\mathrm{loc}}\left(\boldsymbol{F}, \boldsymbol{H}^{i}, \kappa^{i}\right)+\Psi_{\mathrm{nloc}}\left(\boldsymbol{F}, \kappa^{i}, \phi^{i}, \nabla_{\boldsymbol{X}} \phi^{i}\right)
$$

\subsubsection{Total potential energy and variational formulation}

The total potential energy of a system is defined as $\Pi=\Pi_{\text {int }}+\Pi_{\text {ext }}$, where $\Pi_{\text {int }}$ accounts for the contributions due to internal forces and $\Pi_{\text {ext }}=\Pi_{\text {ext }}^{\mathrm{vol}}+\Pi_{\mathrm{ext}}^{\mathrm{sur}}$ accounts for the contributions due to body and surface forces. Given the existence of the strain energy function 
(4.4) and assuming 'dead' loads, each of the contributions to the total potential take the form

$$
\begin{aligned}
\Pi_{\mathrm{int}}\left(\boldsymbol{F}, \boldsymbol{H}^{i}, \kappa^{i}, \phi^{i}, \nabla_{\boldsymbol{X}} \phi^{i}\right) & =\int_{\mathcal{B}_{0}} \Psi_{\mathrm{int}} \mathrm{d} V, \\
\Pi_{\mathrm{ext}}^{\mathrm{vol}}(\boldsymbol{\varphi}) & =\int_{\mathcal{B}_{0}} \Psi_{\mathrm{ext}}^{\mathrm{vol}} \mathrm{d} V=-\int_{\mathcal{B}_{0}} \boldsymbol{B} \cdot \boldsymbol{\varphi} \mathrm{d} V, \\
\Pi_{\mathrm{ext}}^{\mathrm{sur}}(\boldsymbol{\varphi}) & =\int_{\partial \mathcal{B}_{0}} \Psi_{\text {ext }}^{\mathrm{sur}} \mathrm{d} A=-\int_{\partial \mathcal{B}_{0}} \boldsymbol{T} \cdot \boldsymbol{\varphi} \mathrm{d} A,
\end{aligned}
$$

where $\boldsymbol{B}$ denotes the body force vector per unit reference volume and where $\boldsymbol{T}$ characterises the traction vector per unit reference surface area.

The boundary value problem is governed by the principle of minimum potential energy, which requires that the first variation of the total potential energy with respect to $\varphi$ and $\phi^{i}$ vanishes identically, i.e.

$$
\begin{aligned}
\delta_{\boldsymbol{\varphi}} \Pi\left(\boldsymbol{\varphi}, \boldsymbol{F}, \boldsymbol{H}^{i}, \kappa^{i}, \phi^{i}, \nabla_{\boldsymbol{X}} \phi^{i}\right) \doteq \mathbf{0} & \forall \quad \delta \boldsymbol{\varphi}, \\
\delta_{\phi^{i}} \Pi\left(\boldsymbol{\varphi}, \boldsymbol{F}, \boldsymbol{H}^{i}, \kappa^{i}, \phi^{i}, \nabla_{\boldsymbol{X}} \phi^{i}\right) \doteq 0 & \forall \quad \delta \phi^{i} .
\end{aligned}
$$

In detail, the resulting coupled system of equations in material description can be deduced as

$$
\begin{array}{r}
\int_{\mathcal{B}_{0}} \boldsymbol{P}: \nabla_{\boldsymbol{X}} \delta \boldsymbol{\varphi} \mathrm{d} V-\int_{\mathcal{B}_{0}} \boldsymbol{B} \cdot \delta \boldsymbol{\varphi} \mathrm{d} V-\int_{\partial \mathcal{B}_{0}} \boldsymbol{T} \cdot \delta \boldsymbol{\varphi} \mathrm{d} A \doteq 0, \\
\int_{\mathcal{B}_{0}} \boldsymbol{Y}^{i} \cdot \nabla_{\boldsymbol{X}} \delta \phi^{i} \mathrm{~d} V-\int_{\mathcal{B}_{0}} Y^{i} \delta \phi^{i} \mathrm{~d} V \doteq 0,
\end{array}
$$

where the Piola stress $\boldsymbol{P}$ and the vectorial damage quantity $\boldsymbol{Y}^{i}$ are related to flux terms, whereas the body force $\boldsymbol{B}$ and the scalar damage quantity $Y^{i}$ are associated to source terms. They are defined as

$$
\begin{aligned}
\boldsymbol{P} & =\partial_{\boldsymbol{F}} \Psi, & \boldsymbol{B} & =-\partial_{\boldsymbol{\varphi}} \Psi_{\mathrm{ext}}^{\mathrm{vol}}, \\
\boldsymbol{Y}^{i} & =\partial_{\nabla_{\boldsymbol{X}} \phi^{i}} \Psi, & Y^{i} & =-\partial_{\phi^{i}} \Psi,
\end{aligned}
$$

together with $\boldsymbol{T}=-\partial_{\varphi} \Psi_{\text {ext }}^{\text {sur }}$. The corresponding spatial quantities are given by

$$
\begin{aligned}
\boldsymbol{\sigma} & =\boldsymbol{P} \cdot \operatorname{cof}\left(\boldsymbol{F}^{-1}\right), & \boldsymbol{b} & =J^{-1} \boldsymbol{B}, \\
\boldsymbol{y}^{i} & =\boldsymbol{Y}^{i} \cdot \operatorname{cof}\left(\boldsymbol{F}^{-1}\right), & y^{i} & =J^{-1} Y^{i} .
\end{aligned}
$$

Applying relation $\nabla_{\boldsymbol{X}} \delta \boldsymbol{\varphi}=\nabla_{\boldsymbol{x}} \delta \boldsymbol{\varphi} \cdot \boldsymbol{F}$ as well as $\nabla_{\boldsymbol{X}} \delta \phi^{i}=\nabla_{\boldsymbol{x}} \delta \phi^{i} \cdot \boldsymbol{F}$ results in the variational 
forms in spatial description,

$$
\begin{array}{r}
\int_{\mathcal{B}_{t}} \boldsymbol{\sigma}: \nabla_{\boldsymbol{x}} \delta \boldsymbol{\varphi} \mathrm{d} v-\int_{\mathcal{B}_{t}} \boldsymbol{b} \cdot \delta \boldsymbol{\varphi} \mathrm{d} v-\int_{\partial \mathcal{B}_{t}} \boldsymbol{t} \cdot \delta \boldsymbol{\varphi} \mathrm{d} a \doteq 0, \\
\int_{\mathcal{B}_{t}} \boldsymbol{y}^{i} \cdot \nabla_{\boldsymbol{x}} \delta \phi^{i} \mathrm{~d} v-\int_{\mathcal{B}_{t}} y^{i} \delta \phi^{i} \mathrm{~d} v \doteq 0 .
\end{array}
$$

The first terms in equations (4.16) and (4.10) represent the internal virtual work $\delta_{\varphi} W_{\text {int }}=$ $\delta_{\varphi} \Pi_{\text {int }}$, while the remaining terms characterise the external virtual work $\delta_{\varphi} W_{\text {ext }}=-\delta_{\varphi} \Pi_{\text {ext }}$. Consequently, equations (4.16) and (4.17) can be expressed in compact form as

$$
\begin{aligned}
& \delta_{\varphi} W=\delta_{\varphi} W_{\mathrm{int}}-\delta_{\varphi} W_{\mathrm{ext}}=\mathbf{0} \quad \forall \delta \boldsymbol{\varphi}, \\
& \delta_{\phi^{i}} W=\delta_{\phi^{i}} W_{\text {int }}-\delta_{\phi^{i}} W_{\text {ext }}=0 \quad \forall \quad \delta \phi^{i},
\end{aligned}
$$

where the internal and external contributions are given in spatial form as

$$
\begin{aligned}
\delta_{\boldsymbol{\varphi}} W_{\mathrm{int}} & =\int_{\mathcal{B}_{t}} \boldsymbol{\sigma}: \nabla_{\boldsymbol{x}} \delta \boldsymbol{\varphi} \mathrm{d} v \\
\delta_{\boldsymbol{\varphi}} W_{\mathrm{ext}} & =\int_{\mathcal{B}_{t}} \boldsymbol{b} \cdot \delta \boldsymbol{\varphi} \mathrm{d} v+\int_{\partial \mathcal{B}_{t}} \boldsymbol{t} \cdot \delta \boldsymbol{\varphi} \mathrm{d} a \\
\delta_{\phi^{i}} W_{\mathrm{int}} & =\int_{\mathcal{B}_{t}} \boldsymbol{y}^{i} \cdot \nabla_{\boldsymbol{x}} \delta \phi^{i} \mathrm{~d} v \\
\delta_{\phi^{i}} W_{\mathrm{ext}} & =\int_{\mathcal{B}_{t}} y^{i} \delta \phi^{i} \mathrm{~d} v .
\end{aligned}
$$

These relations provide the basis for the finite-element discretisation described in Section 4.3 .

\subsubsection{Strong form - Euler-Lagrange equations}

For the sake of completeness, the corresponding local Euler-Lagrange equations in spatial description are derived in this section. By means of the well-established relations

$$
\begin{aligned}
\boldsymbol{\sigma}: \nabla_{\boldsymbol{x}} \delta \boldsymbol{\varphi} & =\nabla_{\boldsymbol{x}} \cdot[\delta \boldsymbol{\varphi} \cdot \boldsymbol{\sigma}]-\delta \boldsymbol{\varphi} \cdot\left[\nabla_{\boldsymbol{x}} \cdot \boldsymbol{\sigma}\right] \\
\boldsymbol{y}^{i} \cdot \nabla_{\boldsymbol{x}} \delta \phi^{i} & =\nabla_{\boldsymbol{x}} \cdot\left[\delta \phi^{i} \boldsymbol{y}^{i}\right]-\delta \phi^{i} \nabla_{\boldsymbol{x}} \cdot \boldsymbol{y}^{i}
\end{aligned}
$$


and Gauss's divergence theorem applied to (4.16) and (4.17), we obtain the Euler-Lagrange equations in spatial form as

$$
\begin{array}{rlrlr}
\nabla_{\boldsymbol{x}} \cdot \boldsymbol{\sigma}+\boldsymbol{b} & =\mathbf{0} & \text { in } & \mathcal{B}_{t}, \\
\boldsymbol{\sigma} \cdot \boldsymbol{n} & =\boldsymbol{t} & \text { on } & \partial \mathcal{B}_{t}, \\
\text { and } \quad & \nabla_{\boldsymbol{x}} \cdot \boldsymbol{y}^{i}+y^{i}=0 & \text { in } & \mathcal{B}_{t}, \\
\boldsymbol{y}^{i} \cdot \boldsymbol{n} & =0 & \text { on } & \partial \mathcal{B}_{t} .
\end{array}
$$

Equations (4.26), (4.27) represent the common quasi-static balance of linear momentum in local form with respect to the spatial configuration $\mathcal{B}_{t}$. Equations (4.28), (4.29) represent the governing local balance relations associated with the non-local damage field $\phi^{i}$ with respect to the spatial configuration $\mathcal{B}_{t}$.

Remark 4.1.1 It is important to note that-as implied in equation (4.29) - the flux of the non-local damage variable across the boundary $\partial \mathcal{B}_{t}$ is allowed, but it is not defined a priori as a natural boundary condition, as done for instance by de Borst and Pamin [49], Peerlings et al. [108] and Simone et al. [109], amongst others.

\subsection{Constitutive relations}

In this section we introduce the constitutive equations that define the inelastic response at the local material point level. As a key aspect, the gradient-enhanced, non-local contribution to the free energy function is specified, followed by the formulation of continuum damage and its related algorithmic implementation.

\subsubsection{Gradient-enhanced part of the free energy function}

According to equation (4.3), the non-local part of the free energy function of the $i$-th phase is assumed to be additively composed by a gradient-related contribution $\Psi_{\mathrm{grd}}^{i}$ and by a penalty term $\Psi_{\text {plty }}^{i}$. Following Dimitrijević and Hackl [55], who considered an analogous framework for the geometrically linear case, these free energy terms are specified as

$$
\begin{aligned}
\Psi_{\text {grd }}^{i}\left(\boldsymbol{F}, \nabla_{\boldsymbol{X}} \phi^{i}\right) & =\frac{c_{\mathrm{d}}^{i}}{2} \nabla_{\boldsymbol{X}} \phi^{i} \cdot \boldsymbol{C}^{-1} \cdot \nabla_{\boldsymbol{X}} \phi^{i}=\frac{c_{\mathrm{d}}^{i}}{2} \nabla_{\boldsymbol{x}} \phi^{i} \cdot \nabla_{\boldsymbol{x}} \phi^{i}, \\
\Psi_{\mathrm{plty}}^{i}\left(\kappa^{i}, \phi^{i}\right) & =\frac{\beta_{\mathrm{d}}^{i}}{2}\left[\phi^{i}-\kappa^{i}\right]^{2} .
\end{aligned}
$$

The penalty parameter $\beta_{\mathrm{d}}^{i}$ enforces the constraint $\phi^{i}-\kappa^{i}=0$, so that the local and nonlocal damage variables nearly coincide. Furthermore, the gradient parameter $c_{\mathrm{d}}^{i}$ controls the degree of gradient regularisation and the level of smoothing of the non-local damage 
variable over the domain. For the particular non-local free energy functions (4.30) and (4.31), and by using relations (4.13) and (4.15) we obtain

$$
\begin{aligned}
y^{i} & =J^{-1} \beta_{\mathrm{d}}^{i}\left[\phi^{i}-\kappa^{i}\right] \\
\boldsymbol{y}^{i} & =J^{-1} c_{\mathrm{d}}^{i} \nabla_{\boldsymbol{x}} \phi^{i}
\end{aligned}
$$

Based on the general format of the variational form for the non-local damage field variable (4.17) and by using relations (4.32) and (4.33), the Euler-Lagrange equations in the spatial configuration for the non-local damage variable $\phi^{i}$ can be reformulated as

$$
\begin{aligned}
c_{\mathrm{d}}^{i} \Delta_{\boldsymbol{x}} \phi^{i}+\beta_{\mathrm{d}}^{i}\left[\phi^{i}-\kappa^{i}\right] & =0 & \text { in } & \mathcal{B}_{t} \\
\nabla_{\boldsymbol{x}} \phi^{i} \cdot \boldsymbol{n} & =0 & \text { on } & \partial \mathcal{B}_{t}
\end{aligned}
$$

with the Laplace operator $\Delta_{\boldsymbol{x}}(\bullet)=\nabla_{\boldsymbol{x}} \cdot\left[\nabla_{\boldsymbol{x}}(\bullet)\right]$. For further details on the derivation of these equations see Waffenschmidt et al. [110].

\subsubsection{Gradient-enhanced damage model - continuous formula- tion}

In order to obtain the stress-like thermodynamic forces driving the damage process in each $i$-th phase of the fibre-reinforced material, we follow standard thermodynamic arguments; see, e.g., Lemaitre and Chaboche [31]. For the sake of readability, in what follows, no specific super indexing is used to differentiate the quantities related to each phase, provided the same constitutive equations govern the evolution of damage in each of them.

Differentiation of the general format of the free energy function (4.4) with respect to time and application of the Clausius-Planck inequality yields a contribution including the thermodynamic force $q$ conjugate to the damage variable $d$, i.e.

$$
q=-\partial_{d} \Psi=-\partial_{d} \Psi_{\mathrm{loc}}-\partial_{d} \Psi_{\mathrm{nloc}}=q_{\mathrm{loc}}+q_{\mathrm{nloc}}
$$

In this context, note that relation $f_{\mathrm{d}}(\kappa)=1-d$ so that $-\partial_{d}(\bullet)=\partial_{f_{\mathrm{d}}}(\bullet)$. The thermodynamic force $q$, i.e. the effective strain energy or 'energy release rate', is composed of a local and non-local contribution $q_{\mathrm{loc}}=-\partial_{d} \Psi_{\mathrm{loc}}$ and $q_{\mathrm{nloc}}=-\partial_{d} \Psi_{\mathrm{nloc}}=-\partial_{\kappa} \Psi_{\mathrm{nloc}} \partial_{d} \kappa$, respectively. To be specific, we obtain

$$
q_{\mathrm{loc}}=\Psi_{\mathrm{loc}} \quad \text { and } \quad q_{\mathrm{nloc}}=\beta_{\mathrm{d}}[\phi-\kappa] \partial_{d} \kappa
$$

By means of relation (4.34), the non-local energy release rate can alternatively be expressed as $q_{\text {nloc }}=c_{\mathrm{d}} \Delta_{\boldsymbol{x}} \phi \partial_{d} \kappa$. 
We now adopt a damage condition, at any time of the loading process, as

$$
\Phi_{\mathrm{d}}=q-\kappa \leq 0,
$$

where $\Phi_{\mathrm{d}}<0$ refers to the purely elastic case and where $\Phi_{\mathrm{d}}=0$ includes damage evolution. Based on the postulate of maximum dissipation, a constrained optimisation problem involving the Lagrange multiplier $\lambda$ can be constructed which results in the following associated representation of the evolution of the damage variable

$$
\dot{\kappa}=\lambda \frac{\partial \Phi_{\mathrm{d}}}{\partial q}=\lambda \quad \text { with }\left.\quad \kappa\right|_{t=0}=\kappa_{\mathrm{d}}
$$

Initiation as well as termination of damage are assumed to be governed by the KarushKuhn-Tucker complementary conditions

$$
\lambda \geq 0, \quad \Phi_{\mathrm{d}} \leq 0, \quad \lambda \Phi_{\mathrm{d}}=0 .
$$

which, equivalently but more illustratively, can be reformulated as

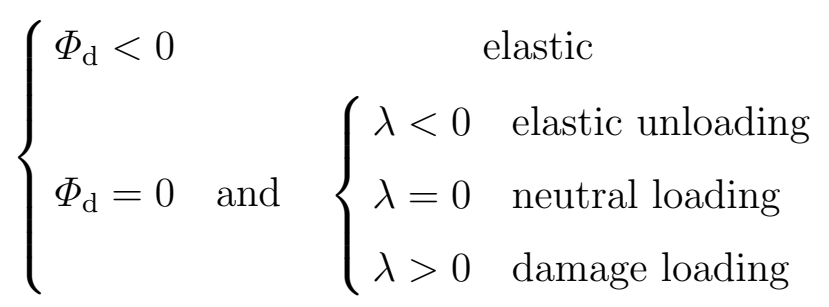

see Simo and Hughes [111] and Figure 4.1 for a graphical representation. For the damage function $f_{\mathrm{d}}(\kappa)$, we adopt a simple exponential-type law

$$
f_{\mathrm{d}}(\kappa)=1-d=\exp \left(\eta_{\mathrm{d}}\left[\kappa_{\mathrm{d}}-\kappa\right]\right)
$$

where we use an initial damage threshold $\kappa_{\mathrm{d}}$, which must be exceeded in order to activate damage evolution. Furthermore, an exponential saturation parameter $\eta_{\mathrm{d}}$ is included. It becomes apparent from this definition that larger values of $\eta_{\mathrm{d}}$ accelerate the damage process, whereas larger values of $\kappa_{\mathrm{d}}$ lead to a delay of the damage initiation.

\subsubsection{Gradient-enhanced damage model - algorithmic setting}

Evolution equation (4.39) represents a non-linear ordinary differential equation, which is integrated numerically in order to obtain the internal damage variable $\kappa$ at a current time step $n+1$. In this context, we introduce representative intervals in time, $\Delta t=t_{n+1}-t_{n} \geq 0$, and apply an implicit, unconditionally stable Euler backward integration scheme, which 


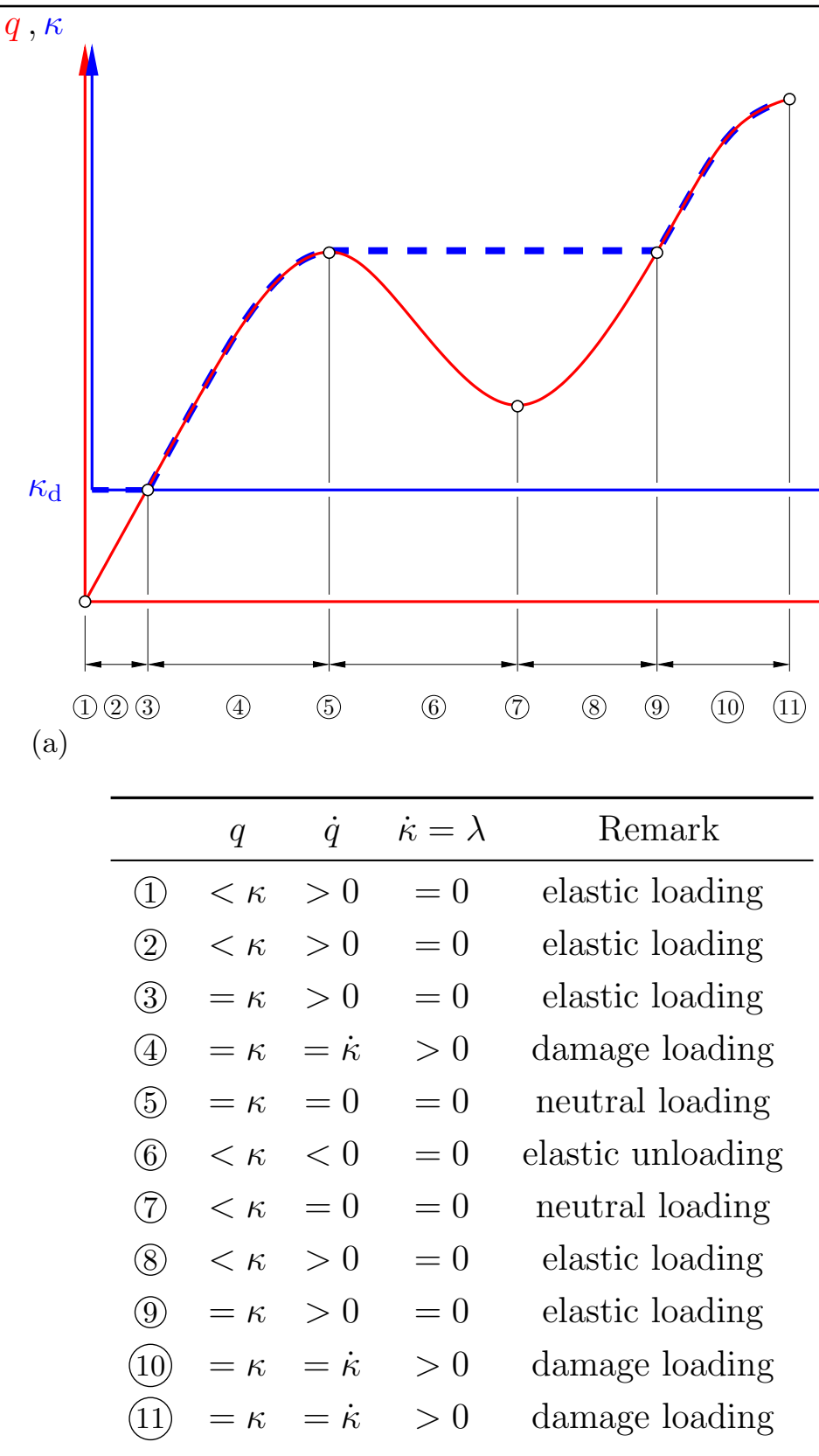

(b)

Figure 4.1: Illustration of the Karush-Kuhn-Tucker conditions, i.e. loading-unloading conditions (4.40) or (4.41) in combination with the consistency condition $\lambda \dot{\Phi}_{\mathrm{d}}=0$.

yields

$$
\kappa_{n+1}=\kappa_{n}+\gamma_{n+1} \quad \text { with }\left.\quad \kappa\right|_{t_{0}}=\kappa_{\mathrm{d}},
$$

where $\gamma_{n+1}:=\Delta t \lambda_{n+1}$ is the incremental Lagrange multiplier at time $t_{n+1}$. The KarushKuhn-Tucker conditions read

$$
\gamma_{n+1} \geq 0, \quad \Phi_{\mathrm{d} n+1} \leq 0, \quad \gamma_{n+1} \Phi_{\mathrm{d} n+1}=0 .
$$


For an inelastic load step, the Lagrange multiplier $\gamma_{n+1}$ is computed by enforcing the consistency condition such that

$$
\begin{aligned}
\Phi_{\mathrm{d} n+1} & =q_{n+1}-\kappa_{n+1}=0, \\
& =\Psi_{n+1}+\frac{\beta_{\mathrm{d}}}{\eta_{\mathrm{d}} f_{\mathrm{d}}\left(\kappa_{n+1}\right)}\left[\phi_{n+1}-\kappa_{n+1}\right]-\kappa_{n+1},
\end{aligned}
$$

which depends non-linearly on $\gamma_{n+1}$. This non-linear equation can be solved iteratively by employing a Newton-Raphson method. Within this iterative scheme the Lagrange multiplier is updated, at every iteration $k$, as

$$
\gamma_{n+1}^{k+1}=\gamma_{n+1}^{k}+\eta \Delta \gamma_{n+1}
$$

with the residual, the Jacobian, and the increment of the Lagrange multiplier defined respectively as

$$
\begin{aligned}
r_{n+1}^{k} & =\Phi_{\mathrm{d} n+1}\left(\kappa_{n+1}^{k}\right), \\
\mathrm{d}_{\gamma} r_{n+1}^{k} & =\frac{\beta_{\mathrm{d}}}{\eta_{\mathrm{d}} f_{\mathrm{d}}\left(\kappa_{n+1}\right)}\left[\eta_{\mathrm{d}}\left[\phi_{n+1}-\kappa_{n+1}\right]-1\right]-1, \\
\Delta \gamma_{n+1} & =-\left[\mathrm{d}_{\gamma} r_{n+1}^{k}\right]^{-1} r_{n+1}^{k} .
\end{aligned}
$$

Within a damped Newton-Raphson scheme, the increment obtained from equation (4.50) can be interpreted as an optimal direction towards the solution. The magnitude of $\Delta \gamma_{n+1}$ of the iteration step is controlled (damped) by the parameter $\eta$ as specified in (4.47). With $\gamma_{n+1}$ determined, the internal damage variable can be updated iteratively according to (4.43). Finally, the stresses can be calculated according to (2.42) and (2.43). The algorithm related to the constitutive model is summarised in Table 4.1. 
0. Given $\boldsymbol{F}_{n+1}, \phi_{n+1}^{i}, \nabla_{\boldsymbol{x}} \phi_{n+1}^{i}$, and $\kappa_{n}^{i}$ at time $t_{n+1}$.

At time $t_{n}$ initialise (non-)local damage field variable as $\left.\kappa^{i}\right|_{t_{0}}=\left.\phi^{i}\right|_{t_{0}}=\kappa_{\mathrm{d}}^{i}$

Loop over the phases $i=0,1,2$

1. Compute $\Psi_{n+1}$ and elastic contributions of Cauchy stress $\overline{\boldsymbol{\sigma}}_{n+1}$ and elasticity tensor $\overline{\mathbf{e}}_{n+1}$

2. Compute driving force $q_{n+1}=\Psi_{n+1}+\frac{\beta_{\mathrm{d}}}{\eta_{\mathrm{d}} f_{\mathrm{d}}\left(\kappa_{n}\right)}\left[\phi_{n+1}-\kappa_{n}\right]$

3. Check damage condition

if $\Phi_{\mathrm{d} n+1}=q_{n+1}-\kappa_{n} \leq 0$, set $\kappa_{n+1}=\kappa_{n}$ and go to 6 .

else go to 5 .

4. Perform local Newton-Raphson scheme to compute $\gamma_{n+1}$ (see Table 4.2)

5. Compute flux and source terms

$$
\begin{aligned}
\boldsymbol{\sigma}_{n+1} & =f_{\mathrm{d}}\left(\kappa_{n+1}\right) \overline{\boldsymbol{\sigma}}_{n+1} \\
\boldsymbol{y}_{n+1} & =J_{n+1}^{-1} c_{\mathrm{d}} \nabla_{\boldsymbol{x}} \phi_{n+1} \\
y_{n+1} & =-J^{-1} \beta_{\mathrm{d}}\left[\phi_{n+1}-\kappa_{n+1}\right]
\end{aligned}
$$

6. Compute tangent moduli

$$
\begin{aligned}
& \mathbf{e}_{n+1}=f_{\mathrm{d}} \overline{\mathbf{e}}_{n+1}+\eta_{\mathrm{d}} f_{\mathrm{d}} \vartheta_{\mathrm{d}} J \overline{\boldsymbol{\sigma}}_{n+1} \otimes \overline{\boldsymbol{\sigma}}_{n+1} \\
& \mathrm{~d} \boldsymbol{\sigma}_{n+1} / \mathrm{d} \phi_{n+1}=2 \mathrm{~d} y_{n+1} / \mathrm{d} \boldsymbol{g}_{n+1}=\beta_{\mathrm{d}} \vartheta_{\mathrm{d}} \boldsymbol{\sigma}_{n+1} \\
& \mathrm{~d} y_{n+1} / \mathrm{d} \phi_{n+1}=-J^{-1} \beta_{\mathrm{d}}\left[1+\beta_{\mathrm{d}}\left[\eta_{\mathrm{d}} f_{\mathrm{d}}\right]^{-1} \vartheta_{\mathrm{d}}\right] \\
& \mathrm{d} \boldsymbol{y}_{n+1} / \mathrm{d} \nabla_{\boldsymbol{x}} \phi_{n+1}=J^{-1} c_{\mathrm{d}} \boldsymbol{I} \\
& \text { with } \vartheta_{\mathrm{d}}=-1-\frac{\eta_{\mathrm{d}} f_{\mathrm{d}}\left(\kappa_{n+1}\right)}{\beta_{\mathrm{d}}\left[\left[1+\eta_{\mathrm{d}} \kappa_{n+1}\right]-\eta_{\mathrm{d}} \phi_{n+1}\right]}
\end{aligned}
$$

End loop over the phases

7. Add up contributions of stress and elasticity tensors

$$
\begin{aligned}
\boldsymbol{\sigma}_{n+1} & =\sum_{i=0,1,2} \boldsymbol{\sigma}_{n+1}^{i} \\
\mathbf{e}_{n+1} & =\sum_{i=0,1,2} \mathbf{e}_{n+1}^{i}
\end{aligned}
$$

Table 4.1: Algorithmic box for the gradient-enhanced damage constitutive model. Expressions used in 6 are derived in Waffenschmidt et al. [110]. 
0. Given: $\Psi_{n+1}$ and $\phi_{n+1}$ at time $t_{n+1}$, and $\kappa_{n}$ at time $t_{n}$

1. Compute residual $r_{n+1}^{k}:=\Phi_{\mathrm{d} n+1}\left(\kappa_{n+1}^{k}\right)$

$$
r_{n+1}^{k}=\Psi_{n+1}+\frac{\beta_{\mathrm{d}}}{\eta_{\mathrm{d}} f_{\mathrm{d}}\left(\kappa_{n+1}^{k}\right)}\left[\phi_{n+1}-\kappa_{n+1}^{k}\right]-\kappa_{n+1}^{k}
$$

2. Check tolerance

if $\left|r_{n+1}^{k}\right|<$ tol go to 6 .

else go to 5.(c)

3. Compute tangent of residual

$\mathrm{d}_{\gamma} r_{n+1}^{k}=\frac{\beta_{\mathrm{d}}}{\eta_{\mathrm{d}} f_{\mathrm{d}}\left(\kappa_{n+1}^{k}\right)}\left[\eta_{\mathrm{d}}\left[\phi_{n+1}-\kappa_{n+1}^{k}\right]-1\right]-1$

4. Compute increment

$\Delta \gamma_{n+1}=-\left[\mathrm{d}_{\gamma} r_{n+1}^{k}\right]^{-1} r_{n+1}^{k}$

5. Update incremental Lagrange multiplier

$\gamma_{n+1}^{k+1}=\gamma_{n+1}^{k}+\eta \Delta \gamma_{n+1}$

with $\eta$ adjusted iteratively (see Table 4.3)

6. Update internal damage variable

$\kappa_{n+1}^{k}=\kappa_{n}+\gamma_{n+1}^{k+1}$

and go to 5.(a)

7. Continue with 5 of Table 4.1

Table 4.2: Calculation steps for the Newton scheme applied for 4 in Table 4.1. 
0. Given: $\kappa_{n}$ at time $t_{n}, \Delta \gamma_{n+1}, \gamma_{n+1}^{k+1}, \gamma_{n+1}^{k}$ and $\eta=1$

1. Evaluate

$$
f_{n+1}^{k}:=\Delta \gamma_{n+1}\left[\Phi_{\mathrm{d} n+1}\left(\kappa_{n}+\gamma_{n+1}^{k}\right)\right]
$$

2. Perform local iteration with adjusted damping parameter $\eta$

(a) Evaluate

$$
f_{n+1}^{k+1}:=\Delta \gamma_{n+1}\left[\Phi_{\mathrm{d} n+1}\left(\kappa_{n}+\gamma_{n+1}^{k+1}\right)\right]
$$

(b) Check condition

if $\left|f_{n+1}^{k+1}\right|<\left|f_{n+1}^{k}\right|$ go to 3 .

else go to (c)

(c) Adjust damping parameter

$\eta \leftarrow \epsilon \eta \quad$ with $\quad \epsilon=0.5$

(d) Adjust update

$$
\gamma_{n+1}^{k+1}=\gamma_{n+1}^{k}+\eta \Delta \gamma_{n+1}
$$

3. Continue with 6 of Table 4.2

Table 4.3: Calculation steps for the damped Newton scheme applied for 5 in Table 4.2.

\subsection{Finite element discretisation}

In this section we present the spatial discretisation of the coupled system of non-linear equations using the finite element method. More specifically, we make use of a coupled Bubnov-Galerkin-based finite element discretisation approach in the spatial configuration, in conjunction with a multivariational formulation to enforce the quasi-incompressibility condition at the material point. The proposed non-local gradient-enhanced damage model is thus implemented by means of an 8-noded Q1P0 hexahedral element for large strains. The motion field $\boldsymbol{\varphi}$ and the non-local damage field $\phi^{i}$ are approximated within the element domain using tri-linear shape functions, whereas the pressure term $\bar{p}$ is assumed to be constant on the element level. The reader should remember that, again, for the sake of readability, no specific super indexing is used to differentiate the non-local damage variable $\phi$ related to each phase $i=\{0,1,2\}$, provided the same discretisation procedure is applied in each of them. The resulting coupled non-linear system of equations can appropriately be solved with an incremental-iterative Newton-Raphson-type solution procedure. 

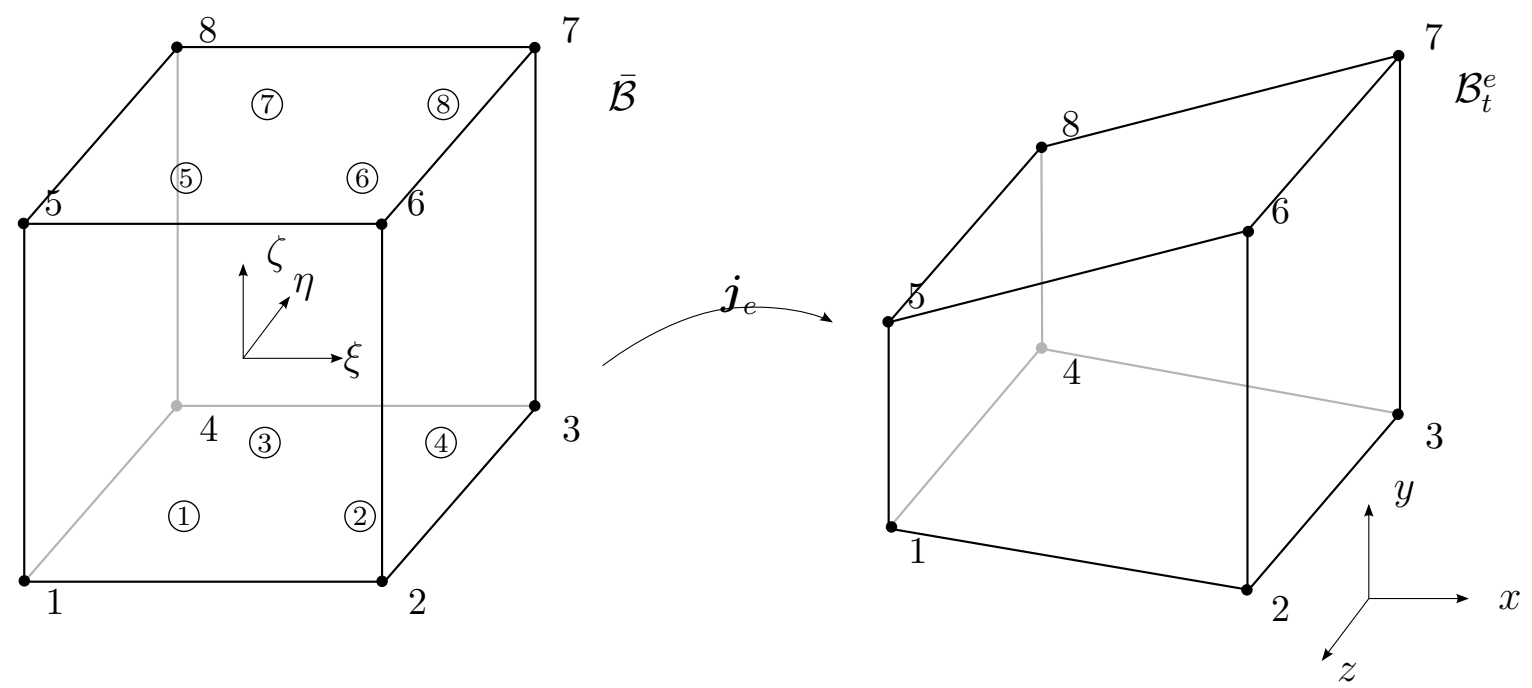

Figure 4.2: Isoparametric 8-noded hexahedral element in reference coordinate space $\{\xi, \eta, \zeta\}$ and in physical coordinate space $\{x, y, z\}$. The integration points are consecutively numbered by (1)(8).

\subsubsection{Discretisation}

The domain $\mathcal{B}_{0}$ is discretised by $n_{\mathrm{el}}$ finite elements, so that

$$
\mathcal{B}_{0} \approx \mathcal{B}_{0}^{\mathrm{h}}=\bigcup_{e=1}^{n_{\mathrm{el}}} \mathcal{B}_{0}^{e}
$$

where every finite element $\mathcal{B}_{0}^{e}$ is characterised by $n_{\mathrm{en}}^{\varphi}$ placement-nodes and $n_{\mathrm{en}}^{\phi}$ non-localdamage-nodes. According to the isoparametric concept, the field variables $\boldsymbol{\varphi}$, as well as the geometry $\boldsymbol{X}$, are interpolated by the same shape functions $N_{I}$, i.e.

$$
\boldsymbol{X} \approx \boldsymbol{X}^{\mathrm{h}}=\sum_{I=1}^{n_{\mathrm{en}}} N_{I}(\boldsymbol{\xi}) \boldsymbol{X}_{I}, \boldsymbol{\varphi} \approx \boldsymbol{\varphi}^{\mathrm{h}}=\sum_{I=1}^{n_{\mathrm{en}}} N_{I}(\boldsymbol{\xi}) \boldsymbol{\varphi}_{I}
$$

and transformed to a hexahedral reference element with coordinates $\boldsymbol{\xi}:=\{\xi, \eta, \zeta\} \in \overline{\mathcal{B}}$, where $\overline{\mathcal{B}}:=\left\{\boldsymbol{\xi} \in \mathbb{R}^{3} \mid-1 \leq \chi \leq+1 ; \chi=\xi, \eta, \zeta\right\}$ denotes the reference domain, see Figure 4.2. In the present context, the number of displacement-nodes and non-local-damagenodes per element are the same, hence $n_{\mathrm{en}}^{\varphi}=n_{\mathrm{en}}^{\phi}=n_{\mathrm{en}}$ and $N_{I}^{\varphi}=N_{I}^{\phi}=N_{I}$. The corresponding field variables, i.e. the placement $\varphi$ and the non-local damage variable $\phi$ as well as their spatial gradients $\nabla_{x} \varphi$ and $\nabla_{x} \phi$, are approximated by means of the product of shape functions $N_{I}(\boldsymbol{\xi})$, and discrete nodal degrees of freedom $\varphi_{I}$ and $\phi_{I}$ summed over $n_{\mathrm{en}}$ 
nodes per element, i.e.

$$
\begin{aligned}
\boldsymbol{\varphi}^{\mathrm{h}} & =\sum_{I=1}^{n_{\mathrm{en}}} N_{I}^{\varphi} \boldsymbol{\varphi}_{I}, & \phi^{\mathrm{h}} & =\sum_{K=1}^{n_{\mathrm{en}}} N_{K}^{\phi} \phi_{K}, \\
\nabla_{\boldsymbol{x}} \boldsymbol{\varphi}^{\mathrm{h}} & =\sum_{I=1}^{n_{\mathrm{en}}} \boldsymbol{\varphi}_{I} \otimes \nabla_{\boldsymbol{x}} N_{I}^{\varphi}, & \nabla_{\boldsymbol{x}} \phi^{\mathrm{h}} & =\sum_{K=1}^{n_{\mathrm{en}}} \phi_{K} \nabla_{\boldsymbol{x}} N_{K}^{\phi} .
\end{aligned}
$$

and an analogous approximation is applied for the variations of field variables and corresponding gradients as

$$
\begin{aligned}
\delta \boldsymbol{\varphi}^{\mathrm{h}} & =\sum_{I=1}^{n_{\mathrm{en}}} N_{I}^{\varphi} \delta \boldsymbol{\varphi}_{I}, & \delta \phi^{\mathrm{h}} & =\sum_{K=1}^{n_{\mathrm{en}}} N_{K}^{\phi} \delta \phi_{K}, \\
\nabla_{\boldsymbol{x}} \delta \boldsymbol{\varphi}^{\mathrm{h}} & =\sum_{I=1}^{n_{\mathrm{en}}} \delta \boldsymbol{\varphi}_{I} \otimes \nabla_{\boldsymbol{x}} N_{I}^{\varphi}, & \nabla_{\boldsymbol{x}} \delta \phi^{\mathrm{h}} & =\sum_{K=1}^{n_{\mathrm{en}}} \delta \phi_{K} \nabla_{\boldsymbol{x}} N_{K}^{\phi},
\end{aligned}
$$

where the spatial and material gradients of the shape functions $N_{I}$ can be expressed as

$$
\begin{aligned}
& \nabla_{\boldsymbol{x}} N_{I}^{\varphi}=\boldsymbol{j}_{e}^{-\mathrm{t}} \cdot \nabla_{\boldsymbol{\xi}} N_{I}^{\varphi} \text { with } \boldsymbol{j}_{e}=\nabla_{\boldsymbol{\xi}} \boldsymbol{\varphi}^{\mathrm{h}}=\sum_{I=1}^{n_{\mathrm{en}}} \boldsymbol{\varphi}_{I} \otimes \nabla_{\boldsymbol{\xi}} N_{I}^{\varphi} \\
& \nabla_{\boldsymbol{X}} N_{I}^{\varphi}=\boldsymbol{J}_{e}^{-\mathrm{t}} \cdot \nabla_{\boldsymbol{\xi}} N_{I}^{\varphi} \text { with } \boldsymbol{J}_{e}=\nabla_{\boldsymbol{\xi}} \boldsymbol{X}^{\mathrm{h}}=\sum_{I=1}^{n_{\mathrm{en}}} \boldsymbol{X}_{I} \otimes \nabla_{\boldsymbol{\xi}} N_{I}^{\varphi}
\end{aligned}
$$

Similar relations for the gradients of $N_{K}^{\phi}$ follow by analogy with (4.57), (4.58). As a consequence, the discrete deformation gradient can be represented in terms of the spatial and material Jacobians of the isoparametric transformation as

$$
\boldsymbol{F}^{\mathrm{h}}:=\nabla_{\boldsymbol{X}} \varphi^{\mathrm{h}}=\boldsymbol{j}_{e} \cdot \boldsymbol{J}_{e}^{-1}
$$

Compatibility provided, the discretised weak form on element level can be written as the difference of the element-specific expressions for the internal and external virtual work related expressions $\delta_{\varphi} W_{\text {int }}^{e}$ and $\delta_{\varphi} W_{\text {ext }}^{e}$, so that

$$
\begin{array}{cccc}
\delta_{\varphi} W^{e}=\delta_{\varphi} W_{\mathrm{int}}^{e}-\delta_{\varphi} W_{\mathrm{ext}}^{e}=\mathbf{0} & \forall & \delta \boldsymbol{\varphi}_{I}, \\
\delta_{\phi} W^{e}=\delta_{\phi} W_{\mathrm{int}}^{e}-\delta_{\phi} W_{\mathrm{ext}}^{e}=0 & \forall & \delta \phi_{K},
\end{array}
$$


where the discrete representations take the following forms

$$
\begin{aligned}
& \delta_{\varphi} W_{\mathrm{int}}^{e}=\sum_{I=1}^{n_{\mathrm{en}}^{\varphi}} \delta \boldsymbol{\varphi}_{I} \cdot \boldsymbol{f}_{\mathrm{int} I}^{\varphi} \quad \text { with } \quad \boldsymbol{f}_{\mathrm{int} I}^{\varphi}=\int_{\mathcal{B}_{t}^{e}} \nabla_{\boldsymbol{x}} N_{I}^{\varphi} \cdot \boldsymbol{\sigma} \mathrm{d} v, \\
& \delta_{\varphi} W_{\mathrm{ext}}^{e}=\sum_{I=1}^{n_{\mathrm{en}}^{\varphi}} \delta \boldsymbol{\varphi}_{I} \cdot \boldsymbol{f}_{\mathrm{ext} I}^{\varphi} \text { with } \boldsymbol{f}_{\mathrm{ext} I}^{\varphi}=\int_{\mathcal{B}_{t}^{e}} N_{I}^{\varphi} \overline{\boldsymbol{b}} \mathrm{d} v \\
& +\int_{\partial \mathcal{B}_{t}^{e}} N_{I}^{\varphi} \overline{\boldsymbol{t}} \mathrm{d} a \\
& \delta_{\phi} W_{\text {int }}^{e}=\sum_{K=1}^{n_{\text {en }}^{\phi}} \delta \phi_{K} f_{\text {int } K}^{\phi} \quad \text { with } f_{\text {int } K}^{\phi}=\int_{\mathcal{B}_{t}^{e}} \nabla_{\boldsymbol{x}} N_{K}^{\phi} \cdot \boldsymbol{y} \mathrm{d} v, \\
& \delta_{\phi} W_{\mathrm{ext}}^{e}=\sum_{K=1}^{n_{\mathrm{en}}^{\phi}} \delta \phi_{K} f_{\mathrm{ext} K}^{\phi} \quad \text { with } f_{\mathrm{ext} K}^{\phi}=\int_{\mathcal{B}_{t}^{e}} N_{K}^{\phi} y \mathrm{~d} v .
\end{aligned}
$$

According to the fundamental lemma of calculus of variations, equations (4.60) and (4.61) are equivalent to the equilibrium of internal and external nodal variables in every $I=$ $1, \ldots, n_{\mathrm{en}}$ placement-node, and $K=1, \ldots, n_{\mathrm{en}}$ non-local-damage-node of a finite element $e$, respectively. This results in the node-specific residual form of the present coupled problem

$$
\begin{aligned}
& \boldsymbol{r}_{I}^{\varphi}=\boldsymbol{f}_{\mathrm{int} I}^{\varphi}-\boldsymbol{f}_{\mathrm{ext} I}^{\varphi}=\mathbf{0} \quad \forall \quad I=1, \ldots, n_{\mathrm{en}}, \\
& r_{K}^{\phi}=f_{\text {int } K}^{\phi}-f_{\text {ext } K}^{\phi}=0 \quad \forall \quad K=1, \ldots, n_{\text {en }} .
\end{aligned}
$$

\subsubsection{Linearisation}

The equations (4.66) and (4.67) represent a coupled and highly non-linear system of equations, hence an iterative Newton-Raphson scheme must be used to find a solution. It must be noted at this point, that the resulting system of equations includes coupling between the motion $\varphi$ and the non-local damage field $\phi$. The non-local damage variable is linked to the local damage variable $\kappa$ by means of the penalty contribution. In what follows and for the sake of readability, we omit the subscript index $n+1$ associated with time $t_{n+1}$. A Taylor series expansion around the solution at the current iteration step $l$-terms of quadratic and higher order being neglected-gives

$$
\begin{gathered}
\boldsymbol{r}_{I l+1}^{\varphi}=\boldsymbol{r}_{I l}^{\varphi}+\Delta \boldsymbol{r}_{I}^{\varphi}=\mathbf{0}, \\
r_{K l+1}^{\phi}=r_{K l}^{\phi}+\Delta r_{K}^{\phi}=0,
\end{gathered}
$$


with the node-specific increments of the residuals

$$
\begin{aligned}
\Delta \boldsymbol{r}_{I}^{\varphi} & =\sum_{J=1}^{n_{\mathrm{en}}^{\varphi}} \frac{\mathrm{d} \boldsymbol{r}_{I}^{\varphi}}{\mathrm{d} \boldsymbol{\varphi}_{J}} \cdot \Delta \boldsymbol{\varphi}_{J}+\sum_{L=1}^{n_{\mathrm{en}}^{\phi}} \frac{\mathrm{d} \boldsymbol{r}_{I}^{\varphi}}{\mathrm{d} \phi_{L}} \Delta \phi_{L}, \\
\Delta r_{K}^{\phi} & =\sum_{J=1}^{n_{\mathrm{en}}^{\phi}} \frac{\mathrm{d} r_{K}^{\phi}}{\mathrm{d} \boldsymbol{\varphi}_{J}} \cdot \Delta \boldsymbol{\varphi}_{J}+\sum_{L=1}^{n_{\mathrm{en}}^{\phi}} \frac{\mathrm{d} r_{K}^{\phi}}{\mathrm{d} \phi_{L}} \Delta \phi_{L} .
\end{aligned}
$$

Herein, the increments $\Delta \boldsymbol{\varphi}_{J}=\boldsymbol{\varphi}_{J l+1}-\boldsymbol{\varphi}_{J l}$ and $\Delta \phi_{L}=\phi_{L l+1}-\phi_{L l}$ represent the difference of the discrete nodal degrees of freedom at iteration-step $l+1$ and $l$. Assuming 'dead loads', the node-specific sub-matrices of the Jacobian are deduced as

$$
\begin{aligned}
\boldsymbol{K}_{I J}^{\varphi \varphi}=\frac{\mathrm{d} \boldsymbol{r}_{I}^{\varphi}}{\mathrm{d} \boldsymbol{\varphi}_{J}}= & \int_{\mathcal{B}_{t}^{e}} \nabla_{\boldsymbol{x}} N_{I}^{\varphi} \cdot \mathbf{e} \cdot \nabla_{\boldsymbol{x}} N_{J}^{\varphi} \mathrm{d} v \\
& +\int_{\mathcal{B}_{t}^{e}}\left[\nabla_{\boldsymbol{x}} N_{I}^{\varphi} \cdot \boldsymbol{\sigma} \cdot \nabla_{\boldsymbol{x}} N_{J}^{\varphi}\right] \boldsymbol{I} \mathrm{d} v, \\
\boldsymbol{K}_{I L}^{\varphi \phi}=\frac{\mathrm{d} \boldsymbol{r}_{I}^{\varphi}}{\mathrm{d} \phi_{L}}= & \int_{\mathcal{B}_{t}^{e}} \nabla_{\boldsymbol{x}} N_{I}^{\varphi} \cdot \frac{\mathrm{d} \boldsymbol{\sigma}}{\mathrm{d} \phi} N_{L}^{\phi} \mathrm{d} v, \\
\boldsymbol{K}_{K J}^{\phi \varphi}=\frac{\mathrm{d} r_{K}^{\phi}}{\mathrm{d} \boldsymbol{\varphi}_{J}}= & \int_{\mathcal{B}_{t}^{e}} N_{K}^{\phi} 2 \frac{\mathrm{d} y}{\mathrm{~d} \boldsymbol{g}} \cdot \nabla_{\boldsymbol{x}} N_{J}^{\varphi} \mathrm{d} v, \\
K_{K L}^{\phi \phi}=\frac{\mathrm{d} r_{K}^{\phi}}{\mathrm{d} \phi_{L}}= & \int_{\mathcal{B}_{t}^{e}} N_{K}^{\phi} \frac{\mathrm{d} y}{\mathrm{~d} \phi} N_{L}^{\phi} \mathrm{d} v \\
& +\int_{\mathcal{B}_{t}^{e}} \nabla_{\boldsymbol{x}} N_{K}^{\phi} \cdot \frac{\mathrm{d} \boldsymbol{y}}{\mathrm{d} \nabla_{\boldsymbol{x}} \phi} \cdot \nabla_{\boldsymbol{x}} N_{L}^{\phi} \mathrm{d} v .
\end{aligned}
$$

Furthermore, e, $\mathrm{d} \boldsymbol{\sigma} / \mathrm{d} \phi, 2 \mathrm{~d} y / \mathrm{d} \boldsymbol{g}, \mathrm{d} y / \mathrm{d} \phi$ and $\mathrm{d} \boldsymbol{y} / \mathrm{d} \nabla_{\boldsymbol{x}} \phi$ denote partitions of the consistent tangent-moduli as specified in A. The resulting system of equations is clearly symmetricsince a variational framework is used-so that $\boldsymbol{K}_{I L}^{\varphi \phi}=\boldsymbol{K}_{K J}^{\phi{ }^{\mathrm{t}}}$ which conveniently enables the use of symmetric equation solvers. The node-specific residual (load) vectors $(4.62)_{2}-$ $(4.65)_{2}$ and the stiffness contributions (4.72)-(4.75) can be summarised as the element-based residual vector, and stiffness matrix

$$
\begin{aligned}
& \boldsymbol{r}_{e}^{\varphi}:=\left[\boldsymbol{r}_{I}^{\varphi}\right] \quad \boldsymbol{r}_{e}^{\phi}:=\left[r_{K}^{\phi}\right] \quad \Delta \boldsymbol{\varphi}_{e}:=\left[\Delta \boldsymbol{\varphi}_{I}\right] \quad \Delta \boldsymbol{\phi}_{e}:=\left[\Delta \phi_{K}\right]
\end{aligned}
$$

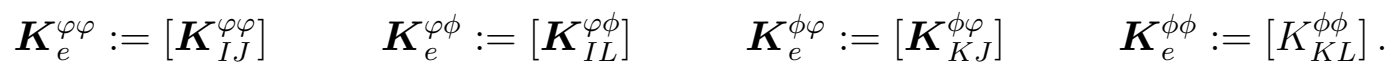

\subsubsection{Assembly of the global system of equations}

Equations (4.69), (4.71), (4.73), (4.74) and (4.75), refer to a single non-local damage variable $\phi$. Nonetheless, when we consider the full set of non-local damage variables $\phi^{i}$ for $i=\{0,1,2\}$, the aforementioned equations still maintain an identical representation for every field $\phi^{i}$ and the linearised system of equations on element level takes the following 
form

$$
\left[\begin{array}{c}
\boldsymbol{r}_{e}^{\varphi} \\
\boldsymbol{r}_{e}^{\phi^{0}} \\
\boldsymbol{r}_{e}^{\phi^{1}} \\
\boldsymbol{r}_{e}^{\phi^{2}}
\end{array}\right]+\left[\begin{array}{cccc}
\boldsymbol{K}_{e}^{\varphi \varphi}+\boldsymbol{K}_{e}^{\mathrm{vol}} & \boldsymbol{K}_{e}^{\varphi \phi^{0}} & \boldsymbol{K}_{e}^{\varphi \phi^{1}} & \boldsymbol{K}_{e}^{\varphi \phi^{2}} \\
\boldsymbol{K}_{e}^{\phi^{0} \varphi} & \boldsymbol{K}_{e}^{\phi^{0} \phi^{0}} & \mathbf{0} & \mathbf{0} \\
\boldsymbol{K}_{e}^{\phi^{1} \varphi} & \mathbf{0} & \boldsymbol{K}_{e}^{\phi^{1} \phi^{1}} & \mathbf{0} \\
\boldsymbol{K}_{e}^{\phi^{2} \varphi} & \mathbf{0} & \mathbf{0} & \boldsymbol{K}_{e}^{\phi^{2} \phi^{2}}
\end{array}\right] \cdot\left[\begin{array}{c}
\Delta \boldsymbol{\varphi}_{e} \\
\Delta \boldsymbol{\phi}_{e}^{0} \\
\Delta \boldsymbol{\phi}_{e}^{1} \\
\Delta \boldsymbol{\phi}_{e}^{2}
\end{array}\right]=\left[\begin{array}{c}
\mathbf{0} \\
\mathbf{0} \\
\mathbf{0} \\
\mathbf{0}
\end{array}\right]
$$

for the determination of the unknown increments of the element degrees of freedom $\Delta \boldsymbol{\varphi}_{e}$, $\Delta \phi^{0}{ }_{e}, \Delta \phi^{1}{ }_{e}$ and $\Delta \phi^{2}{ }_{e}$, being $\boldsymbol{K}_{e}^{\mathrm{vol}}$ a volumetric tangent stiffness matrix that results from the multi-field variation formulation discussed in the following section. Furthermore, the zero matrices appear since there is no coupling amongst the non-local damage variable fields, $\phi^{i}$. Consequently, the assembly of all elements

$\boldsymbol{r}:=\boldsymbol{\bigwedge}_{e=1}^{n_{\mathrm{el}}}\left[\begin{array}{c}\boldsymbol{r}_{e}^{\varphi} \\ \boldsymbol{r}_{e}^{\phi^{0}} \\ \boldsymbol{r}_{e}^{\phi^{1}} \\ \boldsymbol{r}_{e}^{\phi^{2}}\end{array}\right], \quad \boldsymbol{K}=\boldsymbol{A}_{e=1}^{n_{\mathrm{el}}}\left[\begin{array}{cccc}\boldsymbol{K}_{e}^{\varphi \varphi}+\boldsymbol{K}_{e}^{\mathrm{vol}} & \boldsymbol{K}_{e}^{\varphi \phi^{0}} & \boldsymbol{K}_{e}^{\varphi \phi^{1}} & \boldsymbol{K}_{e}^{\varphi \phi^{2}} \\ \boldsymbol{K}_{e}^{\phi^{0} \varphi} & \boldsymbol{K}_{e}^{\phi^{0} \phi^{0}} & \mathbf{0} & \mathbf{0} \\ \boldsymbol{K}_{e}^{\phi^{1} \varphi} & \mathbf{0} & \boldsymbol{K}_{e}^{\phi^{1} \phi^{1}} & \mathbf{0} \\ \boldsymbol{K}_{e}^{\phi^{2} \varphi} & \mathbf{0} & \mathbf{0} & \boldsymbol{K}_{e}^{\phi^{2} \phi^{2}}\end{array}\right], \quad \Delta \boldsymbol{d}=\boldsymbol{\bigwedge}_{e=1}^{\boldsymbol{A}_{\mathrm{el}}}\left[\begin{array}{c}\Delta \boldsymbol{\varphi}_{e} \\ \Delta \boldsymbol{\phi}_{e}^{0} \\ \Delta \boldsymbol{\phi}_{e}^{1} \\ \Delta \boldsymbol{\phi}_{e}^{2}\end{array}\right]$,

results in the global linearised system of equations in the $l$-th iteration step

$$
\boldsymbol{r}_{l}+\boldsymbol{K}_{l} \cdot \Delta \boldsymbol{d}=\mathbf{0} \quad \text { with } \quad \Delta \boldsymbol{d}=\boldsymbol{d}_{l+1}-\boldsymbol{d}_{l}
$$

with $\boldsymbol{K}_{l}$ being the global tangent stiffness matrix, $\Delta \boldsymbol{d}$ the global incremental vector of degrees of freedom, $\boldsymbol{r}_{l}$ the global residual vector including the internal and external system loads and the incorporation of Dirichlet boundary conditions not explicitly indicated here.

Remark 4.3.1 The integrals in equations (4.62)-(4.65) and (4.72)-(4.75) are typically evaluated numerically at discrete integration points by means of appropriate integration or rather quadrature rules, such as Gaussian quadrature, see Hughes [112]. Following de Borst and Pamin [49], we use a reduced $2 \times 2 \times 2$ Gaussian quadrature rule, see Figure 4.2.

\subsubsection{Q1P0 finite element formulation}

The standard displacement based element formulations show very poor performance in convergence, especially when quasi-incompressible material models are used. Several works suggest the use of multivariational approaches to overcome this difficulty. A first work on this regard was presented by Simo et al. [113], where they developed an 8-node hexahedral element for elasto-plasticity at small strains. Nowadays multivariational approaches are commonly used in the simulation of incompressible media at large deformations and in conjunction with different inelastic phenomena, such as plasticity and damage. In this work we implement the proposed non-local gradient-enhanced damage model by means of 
an 8-noded Q1P0 hexahedral element for large strains, i.e. linear shape functions are used for $\varphi$ and $\phi^{i}$, whereas $\bar{p}$ is assumed to be constant on the element level. For the sake of readability, the element will be denoted only as Q1P0. The formulation is based on the introduction of an additional term into the potential energy functional which acts as a constraint imposing the incompressibility via a penalty parameter. Here we limit ourselves to the aspects related to the modification of the tangent stiffness matrix at the element level, and to those related to constitutive response at the local material point level. For a more complete description on the incompressibility topics of the formulation presented in this section, the reader is referred to Wriggers [114] and Miehe [115].

As a starting point we define a new form of the potential (4.5) as

$$
\Pi_{\mathrm{int}}\left(\boldsymbol{F}, \boldsymbol{A}^{i}, \kappa^{i}, \phi^{i}, \nabla_{\boldsymbol{X}} \phi^{i}, p, \theta\right)=\int_{\mathcal{B}_{0}}\left[\Psi_{\mathrm{int}}+p[J-\theta]\right] \mathrm{d} V
$$

where $p$ is a penalty parameter and where $\theta$ is a kinematical variable that measures the volumetric deformation. A consistent linearisation of (4.78), cf. Wriggers [114], shows that these two variables can be discretised consistently in the following manner

$$
\begin{aligned}
& \bar{\theta}=\frac{1}{V^{e}} \int_{\mathcal{B}_{0}} J \mathrm{~d} V \\
& \bar{p}=\frac{1}{V^{e}} \int_{\mathcal{B}_{0}} \Psi^{\prime \mathrm{vol}}(\theta) \mathrm{d} V=\Psi^{\prime \mathrm{vol}}(\theta),
\end{aligned}
$$

where $\Psi^{\prime \text { vol }}$ is the derivative of $\Psi^{\mathrm{vol}}$ with respect to $J$, and which leads to a constant distribution of $\bar{\theta}$ and $\bar{p}$ within the element domain. In that way $\bar{\theta}$ and $\bar{p}$ can be physically interpreted as the mean dilatation and the mean pressure over the element. It becomes apparent from equations (4.79) and (4.80) that the mean dilatation and the mean pressure are computed from the local contributions of the integration points within the element. Finally, the element volumetric tangent stiffness matrix $\boldsymbol{K}_{e}^{\mathrm{vol}}$ is computed following the algorithm defined in Table 4.4. Furthermore, in order to keep consistency with the multivariational formulation, the hydrostatic term of the Cauchy stress tensor should be redefined as

$$
\boldsymbol{\sigma}^{\mathrm{vol}}=\bar{p} \boldsymbol{I}
$$

\subsection{Numerical examples}

In this section we present two boundary value problems that highlight the main capabilities of the proposed gradient-enhanced damage model. We first investigate a homogeneous state under uniaxial tension in order to illustrate the basic constitutive characteristics of the local damage formulation. In a second example we consider a displacement-driven 
0 . Given $\varphi$ at time $t$. Initialize $V^{e}, v^{e}$, and $\nabla_{\boldsymbol{x}} \overline{\boldsymbol{N}}^{\varphi}$.

Loop over integration points gp $=1, \ldots, 8$

1. Compute shape functions, their derivatives in the parametric space, and the transformation map:

$\boldsymbol{N}^{\varphi}, \nabla_{\boldsymbol{\xi}} \boldsymbol{N}^{\varphi}, J_{e}$, and $j_{e}$

2. Compute material and spatial derivatives of the shape functions and the deformation gradient $\boldsymbol{N}^{\varphi}, \nabla_{\boldsymbol{X}} \boldsymbol{N}^{\varphi}, \nabla_{\boldsymbol{x}} \boldsymbol{N}^{\varphi}$, and $\boldsymbol{F}$

3. Compute the initial and current volume $V^{e}=V^{e}+\operatorname{det} J_{e} \omega_{\mathrm{gp}}$ $v^{e}=v^{e}+\operatorname{det} J_{e} \operatorname{det} \boldsymbol{F} \omega_{\mathrm{gp}}$

4. Compute $\nabla_{\boldsymbol{x}} \overline{\boldsymbol{N}}^{\varphi} \nabla_{\boldsymbol{x}} \overline{\boldsymbol{N}}^{\varphi}=\nabla_{\boldsymbol{x}} \overline{\boldsymbol{N}}^{\varphi}+\operatorname{det} J_{e} \operatorname{det} \boldsymbol{F} \omega_{\mathrm{gp}} \nabla_{\boldsymbol{x}} \boldsymbol{N}^{\varphi}$

End loop over integration points

5. Compute $\bar{\theta}, \bar{p}$ and $\boldsymbol{K} i^{\mathrm{vol}}$

$$
\begin{aligned}
\bar{\theta} & =\frac{v^{e}}{V^{e}} \\
\bar{p} & =\Psi^{\prime \mathrm{vol}}(\bar{\theta}) \\
\boldsymbol{K}_{I J}^{\mathrm{vol}} & =\nabla_{\boldsymbol{x}} \bar{N}_{I}^{\varphi} \frac{\Psi^{\prime \prime \mathrm{vol}}(\bar{\theta})}{V^{e}} \nabla_{\boldsymbol{x}} \bar{N}_{J}^{\varphi}
\end{aligned}
$$

Table 4.4: Flowchart for the computation of the volumetric tangent contribution to the element stiffness matrix. $\boldsymbol{N}$ stands for the use of the Voigt notation to refer to the complete set of the shape functions. 
anisotropic plate with a hole. This example serves to illustrate three key aspects: First, the implicit regularisation properties of the model. Second, the effect of the regularisation parameter $c_{\mathrm{d}}^{i}$ on the overall structural response. Third, the independent evolution of the damage variables associated to each $i$-th phase. The proposed gradient-enhanced damage model is implemented in the commercial finite-element software Abaqus/Standard by means of the user subroutine UEL, which allows the implementation of general multi-field non-linear element-formulations. For details see Waffenschmidt et al. [110] or the Abaqus 6.11 Documentation [116].

The damage-related parameters included in constitutive equations (4.30)-(4.31) together with their units are summarised in Table 4.5.

\begin{tabular}{|c|c|c|c|c|c|}
\hline \multirow{2}{*}{ Type } & & \multirow{2}{*}{ Symbol } & \multicolumn{2}{|c|}{ Value } & \multirow{2}{*}{ Unit } \\
\hline & & & Test 1 & Test 2 & \\
\hline \multirow{3}{*}{ Structure } & & $\beta^{1}$ & 0.0 & 0.0 & {$[\mathrm{deg}]$} \\
\hline & & $\beta^{2}$ & 0.0 & 90.0 & [deg] \\
\hline & & $\varkappa$ & 0.0 & 0.0 & {$[-]$} \\
\hline \multirow{6}{*}{ Elasticity } & \multirow{2}{*}{ Matrix } & $\mu_{\mathrm{e}}$ & 15.0 & 15.0 & {$[\mathrm{kPa}]$} \\
\hline & & $\kappa_{\mathrm{e}}$ & 150.0 & 150.0 & {$[\mathrm{kPa}]$} \\
\hline & \multirow{4}{*}{ Fibres } & $k_{1}^{1}$ & 7.5 & 20.0 & {$[\mathrm{kPa}]$} \\
\hline & & $k_{2}^{1}$ & 0.1 & 2.1 & {$[-]$} \\
\hline & & $k_{1}^{2}$ & 7.5 & 20.0 & {$[\mathrm{kPa}]$} \\
\hline & & $k_{2}^{2}$ & 0.1 & 2.1 & {$[-]$} \\
\hline \multirow{4}{*}{ Damage } & \multirow{2}{*}{ Matrix } & $\eta_{\mathrm{d}}^{0}$ & - & 2.0 & {$\left[\mathrm{kPa}^{-1}\right]$} \\
\hline & & $\kappa_{\mathrm{d}}^{0}$ & - & 2.0 & {$[\mathrm{kPa}]$} \\
\hline & \multirow{2}{*}{ Fibres $(i=1,2)$} & $\eta_{\mathrm{d}}^{i}$ & Variable & 7.0 & {$\left[\mathrm{kPa}^{-1}\right]$} \\
\hline & & $\kappa_{\mathrm{d}}^{i}$ & Variable & 0.5 & {$[\mathrm{kPa}]$} \\
\hline \multirow{4}{*}{ Regularisation } & \multirow{2}{*}{ Matrix } & $c_{\mathrm{d}}^{0}$ & - & 250.0 & {$\left[\mathrm{kPa}^{-1} \mathrm{~mm}^{2}\right]$} \\
\hline & & $\beta_{\mathrm{d}}^{0}$ & - & 1000.0 & {$\left[\mathrm{kPa}^{-1}\right]$} \\
\hline & \multirow{2}{*}{ Fibres $(i=1,2)$} & $c_{\mathrm{d}}^{i}$ & - & 1000.0 & {$\left[\mathrm{kPa}^{-1} \mathrm{~mm}^{2}\right]$} \\
\hline & & $\beta_{\mathrm{d}}^{i}$ & - & 1000.0 & {$\left[\mathrm{kPa}^{-1}\right]$} \\
\hline
\end{tabular}

Table 4.5: Set of geometrical, structural and material parameters used in the boundary value problems. Only structural and material parameters are provided. For details on the geometry of the plate see Figure 4.4a. Parameters $\beta^{1}$ and $\beta^{2}$ measure the angle of material vectors $\boldsymbol{a}_{0}^{1}$ and $\boldsymbol{a}_{0}^{2}$ with respect to the cartesian axis $X$.

\subsubsection{Test 1 - Homogeneous uniaxial tension}

As an introductive numerical example to emphasise the properties of the basic constitutive characteristics of the local damage formulation, we next discuss a deformation under 
isochoric uniaxial tension with

$$
\boldsymbol{F}=\lambda_{1} \boldsymbol{e}_{1} \otimes \boldsymbol{e}_{1}+\lambda_{1}^{-1 / 2}\left[\boldsymbol{e}_{2} \otimes \boldsymbol{e}_{2}+\boldsymbol{e}_{3} \otimes \boldsymbol{e}_{3}\right]
$$

The frame $\left\{\boldsymbol{e}_{1}, \boldsymbol{e}_{2}, \boldsymbol{e}_{3}\right\}$ represents a fixed orthonormal base system. A monotonically increasing load is applied up to an axial stretch of $\lambda_{1}=2.0$, where the neo-Hookean matrix remains elastic throughout the deformation. With regard to the structural properties, both families of fibers are oriented with respect to the axial $\boldsymbol{e}_{1}$-direction, i.e. $\beta_{1,2}=0.0$ [deg] where $\beta_{1,2}=\angle\left(\boldsymbol{a}_{01,2}, \boldsymbol{e}_{1}\right)$. As the dispersion parameter $\varkappa$ is set to zero, transverse isotropy is recovered for every fibre family. The material parameters used for this example are summarised in Table 4.5 in column 'Test 1'.

For a homogeneous deformation, the gradient-related free energy term (4.30) is not activated, i.e. $\nabla_{\boldsymbol{x}} \phi^{1}=\nabla_{\boldsymbol{x}} \phi^{2}=\mathbf{0}$. In consequence, the driving force is represented only by the local term $q^{1}=q^{2}=q_{\text {loc }}$ and the damage model's behaviour is in accordance with a purely local model. Therefore, only the influence of the local damage parameters, i.e. the threshold $\kappa_{\mathrm{d}}^{1}$ and the saturation parameter $\eta_{\mathrm{d}}^{1}$, is studied in Figure 4.3, where the left column $4.3(\mathrm{a}, \mathrm{c}, \mathrm{e})$ is associated with values of $\kappa_{\mathrm{d}}^{1}=0.0[\mathrm{kPa}]$, and where the right column $4.3(\mathrm{~b}, \mathrm{~d}, \mathrm{f})$ corresponds to values of $\kappa_{\mathrm{d}}^{1}=16.0[\mathrm{kPa}]$. The stress-stretch response is shown in Figure 4.3a and 4.3b where $P_{11}=\lambda_{1}^{-1} \boldsymbol{e}_{1} \cdot \boldsymbol{\sigma} \cdot \boldsymbol{e}_{1}$. The two solid black curves represent the fully elastic response of two extreme cases: the isotropic neo-Hookean matrix in the lower solid black curve, and the overall elastic response composed of the isotropic neo-Hookean and the anisotropic exponential part, in the upper solid black curve. The inelastic damagerelated load-displacement curves can be seen between the two solid black curves. Basically, the material first shows an elastic response according to the exponential constitutive relation. After a certain loading level, the material softens and deviates from the elastic path. Caused by the loss of stiffness, the stress starts to decrease with increasing stretch $\lambda_{1}$ and the curve finally drops towards the black curve which represents the elastic response of the undamaged neo-Hookean ground substance.

It becomes apparent that higher damage saturation values $\eta_{\mathrm{d}}^{1}$ accelerate the damage process. Generally, it is expected that the purely neo-Hookean response is recovered upon complete damage of the fibers. This can be clearly verified for damage saturation values of $\eta_{\mathrm{d}}^{1}=\left\{1,10^{-1}\right\}\left[\mathrm{kPa}^{-1}\right]$, whereas for saturation values of $\eta_{\mathrm{d}}^{1}=\left\{10^{-2}, 10^{-3}\right\}\left[\mathrm{kPa}^{-1}\right]$ complete damage of the fibers cannot be obtained within the deformation range considered. Furthermore, we observe that the higher damage threshold of $\kappa_{\mathrm{d}}^{1}=16.0[\mathrm{kPa}]$ leads to a delay of the damage initiation.

The influence of the damage threshold $\kappa_{\mathrm{d}}^{1}$ is illustrated in Figures $4.3 \mathrm{c}$ and $4.3 \mathrm{~d}$ where the evolution of the internal damage variable $\kappa$ is shown. Here, we clearly observe how $\kappa_{\mathrm{d}}^{1}$ controls the onset of damage for $\kappa_{\mathrm{d}}^{1}=0.0[\mathrm{kPa}]$. Damage starts at the very beginning 
of the deformation, see Figure 4.3c, and continuously evolves, i.e. $\dot{\kappa}^{1}>0$, according to $\kappa=q^{\text {loc }}=\Psi^{\text {ani }}$. The evolution of $\mathrm{d}^{1}$ ot $\kappa>0$ continues until the fibre-reinforced material is completely damaged. For $\kappa_{\mathrm{d}}^{1}=16.0[\mathrm{kPa}]$, see Figure $4.3 \mathrm{~d}$, the damage process is delayed and starts once the driving force $q^{\text {loc }}=\Psi^{\text {ani }}$ exceeds the damage threshold value of $\kappa_{\mathrm{d}}^{1}=$ $16.0[\mathrm{kPa}]$, i.e. $q^{\text {loc }}>\kappa_{\mathrm{d}}^{1}$. After initiation of the damage process, the damage evolution as represented by $\kappa^{1}>0$ continues, up to complete damage of the fibre-reinforced material.

Figures 4.3e and 4.3f highlight the evolution of the damage function $f_{\mathrm{d}}^{1}$, see equation (4.42). By comparing Figures 4.3e and 4.3f, it becomes apparent how $\kappa_{\mathrm{d}}^{1}$ controls the onset of damage. Furthermore, we clearly observe that damage evolves faster for higher values of $\eta_{\mathrm{d}}^{1}$.

\subsubsection{Test 2 - Plate with a hole}

A first inhomogeneous deformation test is presented in this section, where we study the deformation of a plate with a hole subjected to inhomogeneous Dirichlet boundary conditions. The fibres lie in the $X Y$ plane and are perfectly aligned with the cartesian axes (see Figure 4.4a). The material parameters used for the computation are summarised in Table 4.5. Two scenarios are studied. First a situation where the plate is uniaxially stretched along the $Y$-direction, causing damage to evolve initially in the second fibre family and subsequently in the matrix. Secondly, we consider a situation where the plate is biaxially loaded by means of a combination of inhomogeneous Dirichlet boundary conditions along the $Y$ and $X$-direction, causing damage to evolve in both fibre families as well as in the matrix. These two boundary value problems are introduced in more detail in the following sections. It must be noted that due to the orientation of the fibre reinforcements and the particular combination of boundary conditions only $1 / 8$ of the total geometry of the plate is discretised.

\section{Plate with a hole under uniaxial loading}

In the first example, the plate displayed in Figure 4.4a is loaded uniaxially by clamping the bottom face and imposing inhomogeneous Dirichlet boundary conditions at the top face of the plate. Here, the arc-length method is used in order to capture the snap-back that occurs in the load-displacement response (See Waffenschmidt et al. [110]). The material parameters defining the onset of damage evolution, i.e. $\kappa_{0}^{i}$, have been chosen in such a way that damage is first triggered in the fibres and later on in the matrix.

This boundary value problem illustrates three key aspects of the proposed model, namely the implicit regularisation properties of the model, the effect of the associated regularisation parameter on the overall structural response, and the independent evolution of the damage variables associated to each $i$-th phase. Several references can be found in the literature 

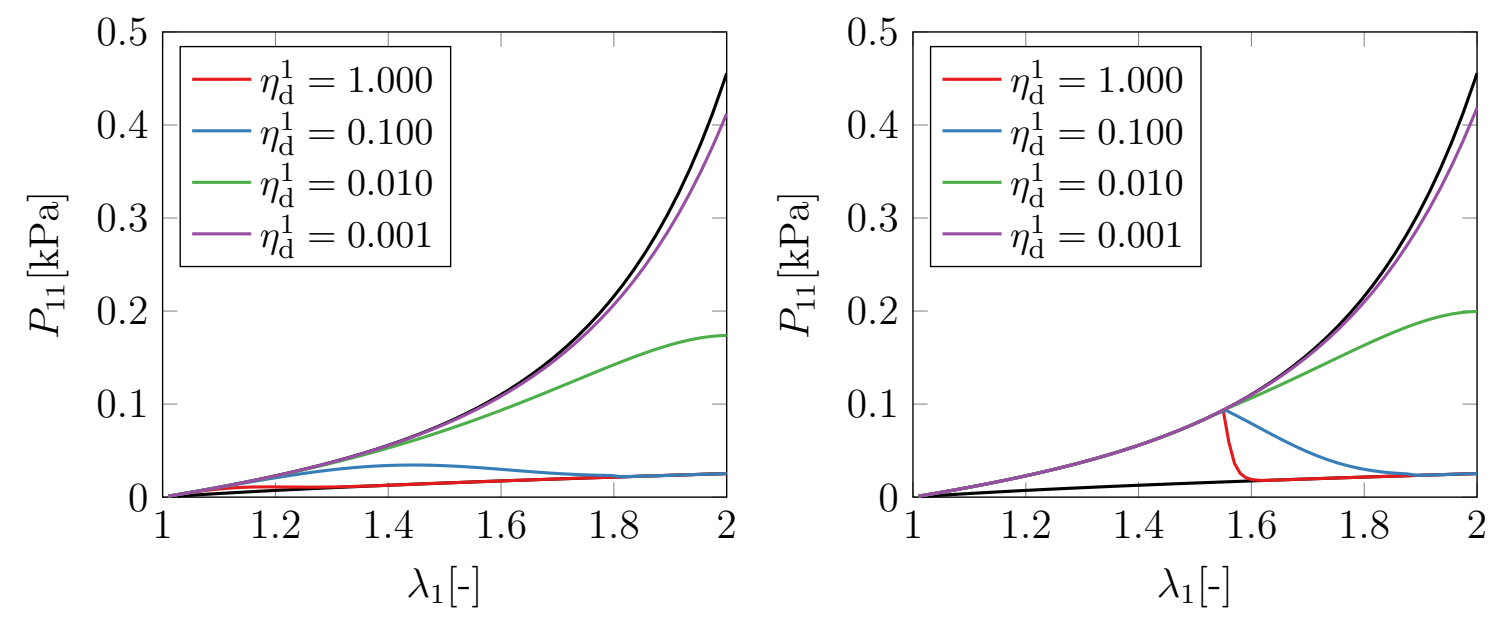

(a)

(b)
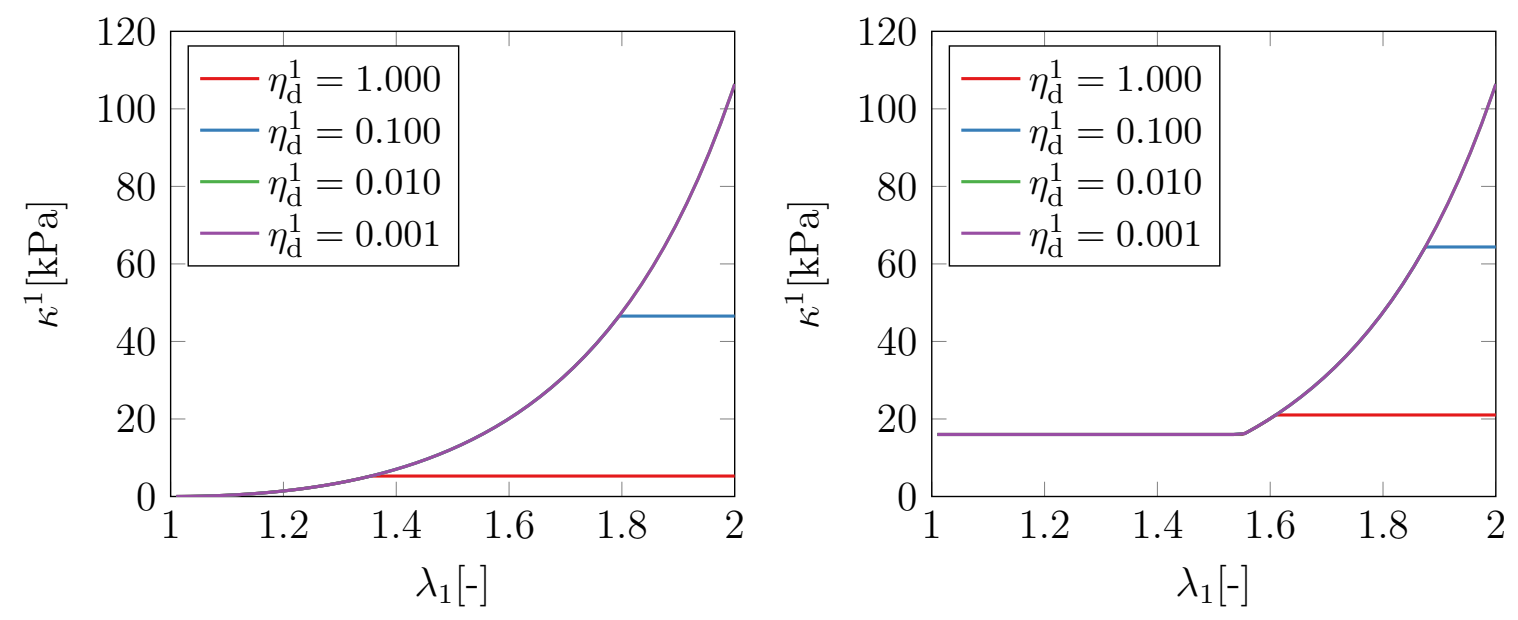

(c)

(d)
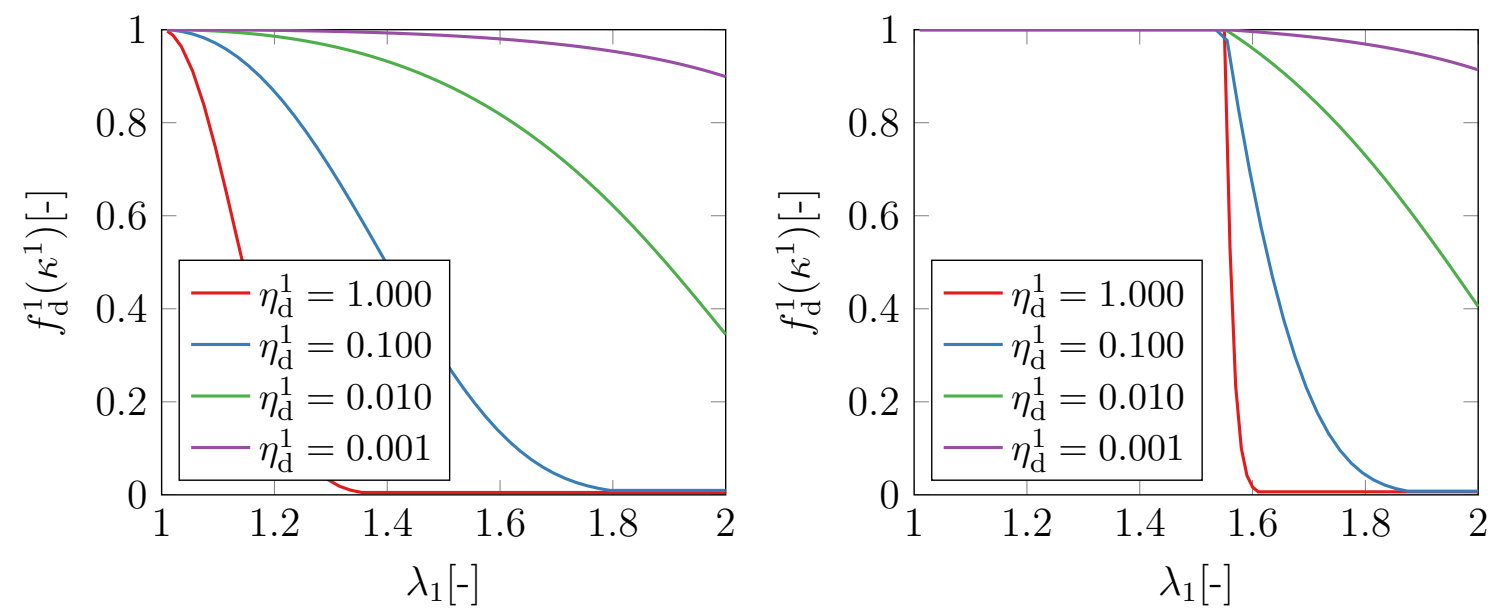

(e)

(f)

Figure 4.3: Uniaxial isochoric tension: Influence of the saturation parameter $\eta_{\mathrm{d}}^{1}$. Subfigures (a,c,e) correspond to $\kappa_{\mathrm{d}}^{1}=0.0[\mathrm{kPa}]$. Subfigures $(\mathrm{b}, \mathrm{d}, \mathrm{f})$ correspond to $\kappa_{\mathrm{d}}^{1}=16.0[\mathrm{kPa}]$. The units of the saturation parameter $\eta_{\mathrm{d}}^{1}$ are given in $\left[\mathrm{kPa}^{-1}\right]$. 


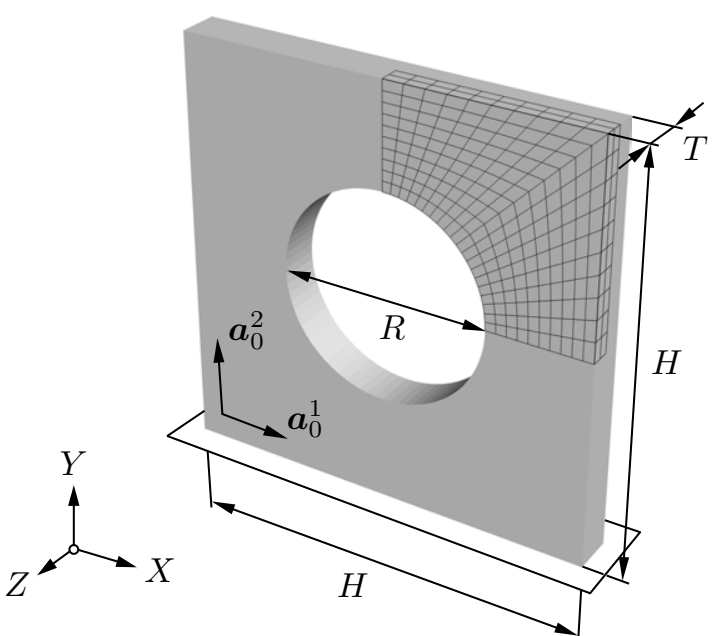

(a)

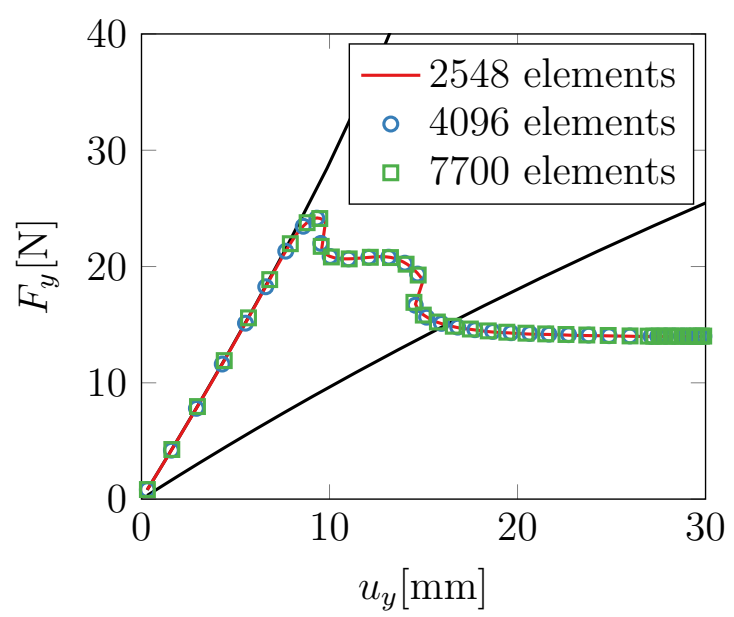

(b)

Figure 4.4: Plate with a hole. (a) Dimensions ( $R=100[\mathrm{~mm}], H=200[\mathrm{~mm}], T=20[\mathrm{~mm}])$, orthotropic fibre orientations $\boldsymbol{a}_{0}^{1}, \boldsymbol{a}_{0}^{2}$ and discretisation (1/8 of the full geometry). (b) Forcedisplacement response and investigation of mesh-sensitivity. The solid black curves represent the elastic response of the isotropic neo-Hookean matrix (lower curve) and the overall elastic response of the fibre-reinforced material (upper curve).

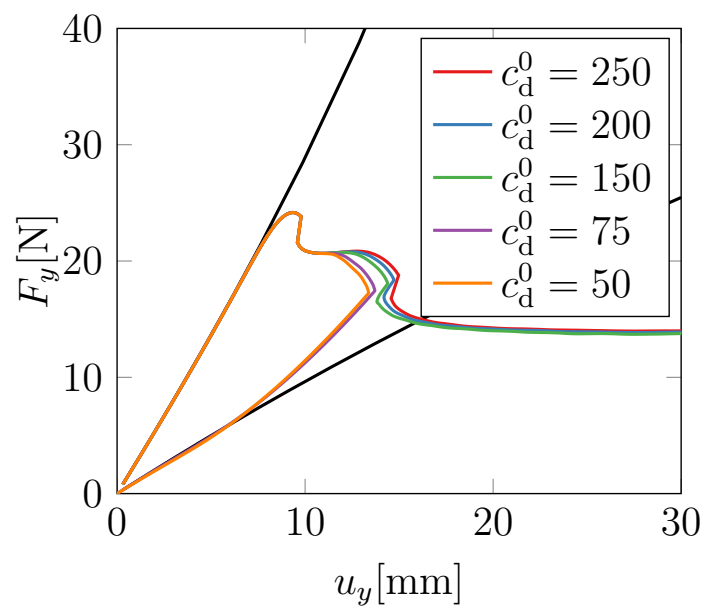

(a)

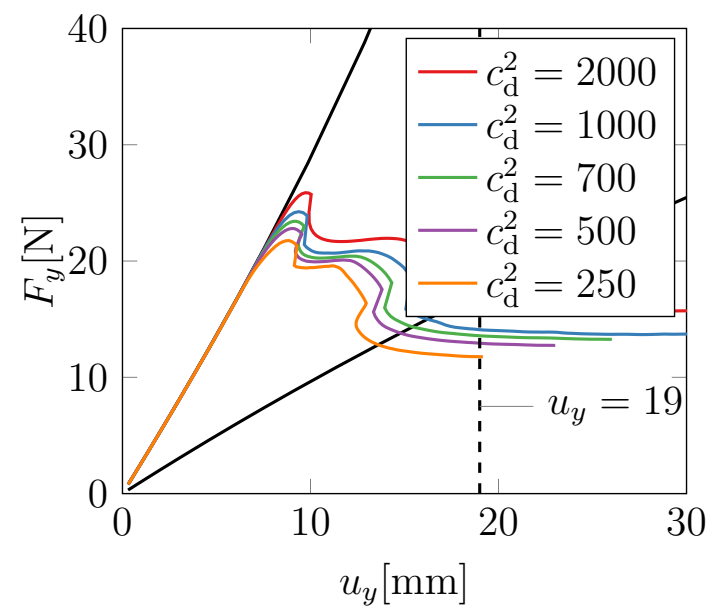

(b)

Figure 4.5: Load-displacement response of a plate with a hole under uniaxial loading. The solid black curves represent the elastic response of the isotropic neo-Hookean matrix (lower curve) and the overall elastic response of the fibre-reinforced material (upper curve). (a) Investigation of the influence of the regularisation parameter $c_{\mathrm{d}}^{0}$. (b) Investigation of the influence of the regularisation parameter $c_{\mathrm{d}}^{2}$. Units of $c_{\mathrm{d}}^{i}$ are in $\left[\mathrm{kPa}^{-1} \mathrm{~mm}^{2}\right]$, as specified in Table 4.5 .

which advert that one of the major drawbacks of purely local damage formulations is the fact that damage tends to be localised within only one single row of elements upon mesh refinement causing dissipation to be mesh-dependent, which is not physically reasonable. In contrast to local damage formulations the proposed non-local gradient-enhanced damage model guarantees that, for sufficiently large regularisation parameters $c_{\mathrm{d}}$, dissipation 


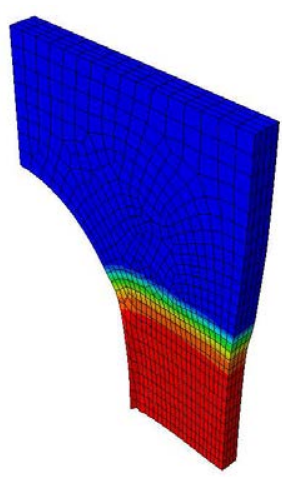

(a)

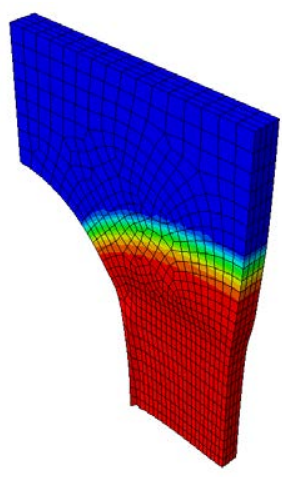

(e)

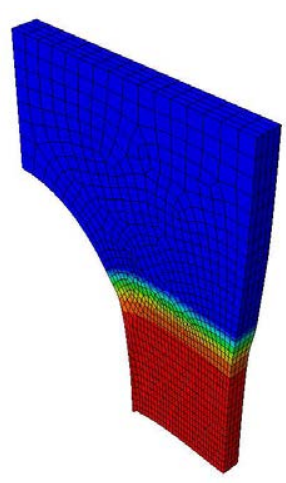

(b)

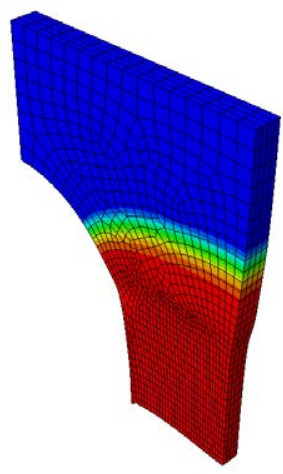

(f)

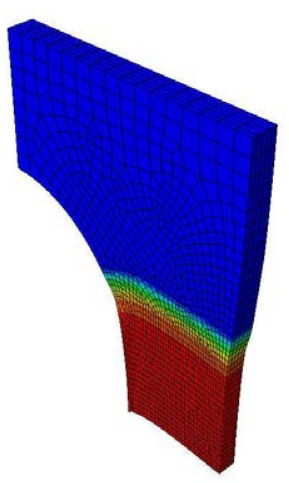

(c)

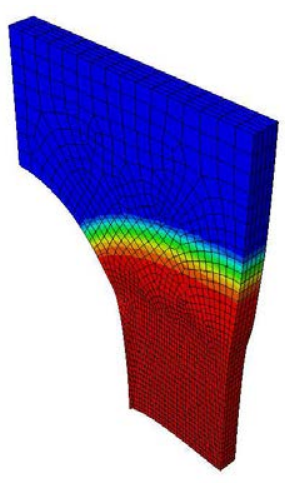

$(\mathrm{g})$
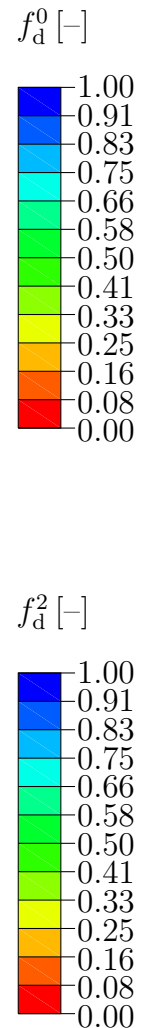

Figure 4.6: Plate with a hole under uniaxial loading. Contour plots of the damage function $f_{\mathrm{d}}^{0}$ (first row) and damage function $f_{\mathrm{d}}^{2}$ (second row)for three different discretisations at $u_{y}=30[\mathrm{~mm}]$. (a,e) 2548 elements, (b,f) 4096 elements, (c,g) 7700 elements. 


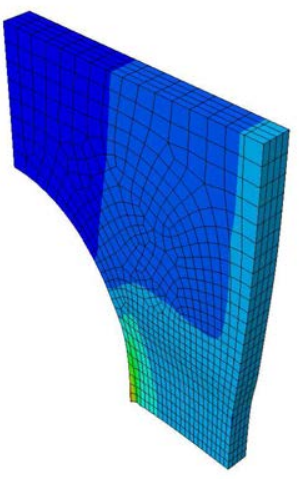

(a)

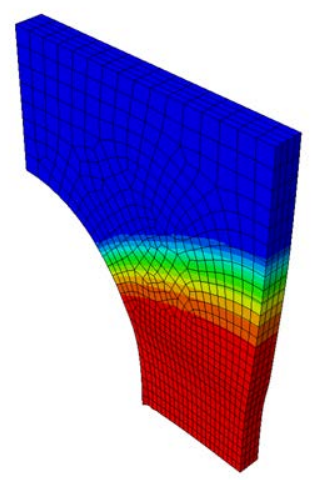

(e) (f)

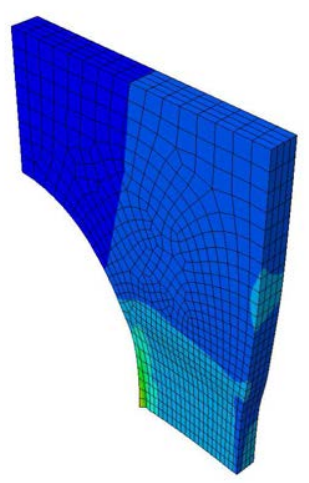

(b)

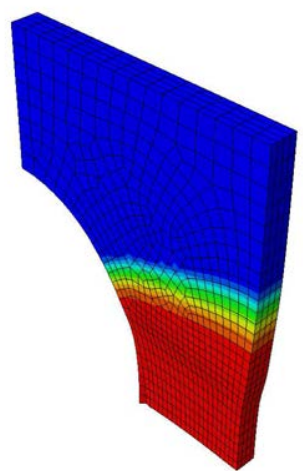

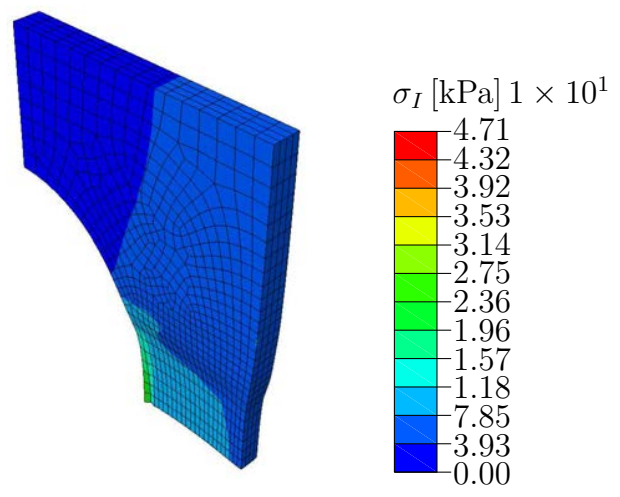

(c)
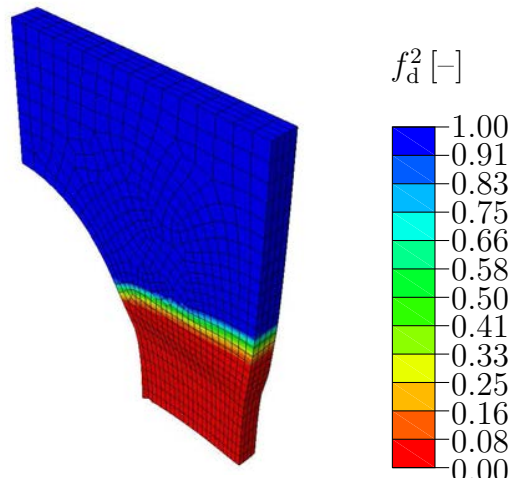

(g)

Figure 4.7: Plate with a hole under uniaxial loading. Contour plots of the damage function $f_{\mathrm{d}}^{2}$ for different values of regularisation at $u_{y}=19.0[\mathrm{~mm}]$ (see Figure $4.5 \mathrm{~b}$ ). (a) $c_{\mathrm{d}}^{2}=2000\left[\mathrm{kPa}^{-1} \mathrm{~mm}^{2}\right]$, (b) $c_{\mathrm{d}}^{2}=1000\left[\mathrm{kPa}^{-1} \mathrm{~mm}^{2}\right]$, (c) $c_{\mathrm{d}}^{2}=250\left[\mathrm{kPa}^{-1} \mathrm{~mm}^{2}\right]$ 
remains constant for any discretisation, hence ensuring that the results of the simulations are mesh-objective.

Three different non-structured meshes (2548, 4096 and 7700 elements) are used to study the mesh-objectivity. The discretisation is refined close to the bottom of the plate, where the localisation of damage is expected to take place. Figure $4.4 \mathrm{~b}$ shows the structural response obtained for the different discretisations. First we focus on the elastic response of the structure which is represented by the solid black curves. The lower black curve corresponds to the neo-Hookean material and the upper black curve to the fibre-reinforced material. Since the second fibre family is perfectly aligned with the stretching direction $Y$, a sharp difference between the neo-Hookean and the fibre-reinforced material responses can be appreciated from the very beginning.

Regarding the evolution of damage, we observe that the load-displacement curves follow the path of the elastic fibre-reinforced material up to the point where damage is triggered in the second fibre family. At that moment a loss of stiffness in the structure causes the curves to deviate from the elastic response and later on a snap-back appears while elastic energy is released in the upper side of the plate. A second drop in the load-displacement curves appear as damage is triggered in the matrix which is followed by another snap-back when more elastic energy is released. The results in Figure 4.4b show how the implicit regularisation of the proposed model ensures the mesh-objectivity. Moreover, the contour plots of the damage functions $f_{\mathrm{d}}^{0}$ and $f_{\mathrm{d}}^{2}$ in Figure 4.6 show a good match in the non-local damage variable field over the whole domain for the different discretisations.

The regularisation parameter $c_{\mathrm{d}}$ on the structural response of the plate is studied in Figure 4.5 a for different values of $c_{\mathrm{d}}^{0}$ while keeping $c_{\mathrm{d}}^{2}=1000\left[\mathrm{kPa}^{-1} \mathrm{~mm}^{2}\right]$ fixed. We first observe that the response of the structure does not change along the first drop of the load-displacement curve. This first drop corresponds to the initiation of damage in the fibre-reinforcements. Hence, the parameter $c_{\mathrm{d}}^{0}$ which is associated to the matrix, has no influence in the structural response of the plate during this stage. Nonetheless, as soon as damage is initiated in the matrix, we observe that lower values of $c_{\mathrm{d}}^{0}$ entail a reduction in the load-bearing capacity due to the reduced regularisation. A more straight-forward interpretation can be drawn from the contour plots of the damage function in Figure 4.7. The regularisation parameter controls the degree of homogeneity of the solution in the non-local damage variable field. Larger values of the regularisation parameter render more uniform solutions in the non-local damage variable, while lower values favour the localisation of damage.

Another interesting aspect that is highlighted in Figure $4.5 \mathrm{a}$ is the fact that for $c_{\mathrm{d}}^{0}=$ $[75.0,50.0]\left[\mathrm{kPa}^{-1} \mathrm{~mm}^{2}\right]$ the solution does not progress further in the displacement after the second snap-back. There are two possible explanations for this behaviour. A first explanation arises from a technical detail. Due to the fact that we use the arc-length method for these simulations, the Dirichlet boundary conditions are weighted by a load proportionality 
factor $\lambda$ which, itself, an unknown of the problem. From the results we can simply conclude that the solver does not find values of $\lambda$ that favour the progress of the solution into the inelastic loading. A second explanation which has a more physically sound foundation can be drawn from the following consideration. We have already established that lower values of $c_{\mathrm{d}}$ favour the localisation of damage, which in turn reduce the dissipation in the structural response. Energy dissipation is the mechanism that allows a structure to reach high states of deformation while following a load-displacement path in a quasi-static state. An example that serves to illustrate this point is the comparison of failure in quasi-brittle materials (i.e. concrete), which dissipate little energy, and failure in ductile materials (i.e. steel), which dissipate more energy. Typical load-displacement curves from experiments in quasi-brittle materials show the occurrence of sharp snap-backs in the structural response, which are usually followed by a sudden crack of the structure or a short progression in the inelastic loading. However, a full development of the load-displacement curve up to the point of complete material failure is not possible in a quasi-static state. In contrast to quasi-brittle materials, ductile materials show typically larger displacements/deformations along the inelastic loading path precisely because they dissipate more energy. From the results we have obtained it could be suggested that, for low values of the regularisation parameter $c_{\mathrm{d}}$, a further progression in the load-displacement curve cannot be achieved simply because the dissipation is not high enough to allow the occurrence of the inelastic loading in a quasi-static state. A more comprehensive analysis on the issue of energy dissipation and its effect on the mechanical behaviour of structures can be found in de Borst [117].

We believe that these two hypotheses are closely related and that the reason for the arclength method not to find values for the load proportionality factor $\lambda$ that allow for the evolution in the inelastic loading regime, is due to the fact that dissipation has reached a critical lower-bound.

To conclude this first example, we turn our attention to Figure 4.5b, where we see the effect of the $c_{\mathrm{d}}^{2}$ parameter on the structural response of the plate while keeping $c_{\mathrm{d}}^{0}=250$ fixed. The same conclusions drawn from the variation of the regularisation parameter in matrix $c_{\mathrm{d}}^{0}$ (see Figure 4.5a) can be applied here. Lower values of $c_{\mathrm{d}}$ entail a reduction in the energy dissipation, but in contrast to the results presented in Figure 4.5a, Figure 4.5b clearly shows a reduction in the load-bearing capacity in the structure. This occurs because the peak load is achieved during the first stage in which damage evolves only in the second fibre family, and since $c_{\mathrm{d}}^{2}$ precisely affects the dissipation in these fibres, any reduction in $c_{\mathrm{d}}^{2}$ entails a reduction in the overall load-bearing capacity of the structure. A different situation emerges when $c_{\mathrm{d}}^{2}$ is increased. In Figure $4.5 \mathrm{~b}$ we see that, when we increase the value of regularisation up to $c_{\mathrm{d}}^{2}=2000\left[\mathrm{kPa}^{-1} \mathrm{~mm}^{2}\right]$, the peak load of the structure is increased in comparison to the peak load in Figure 4.5a. Finally, the computations for $c_{\mathrm{d}}^{2}=[700,500,250]$ do not reach the maximum imposed displacement $u=30[\mathrm{~mm}]$ because 
(1)-(2)

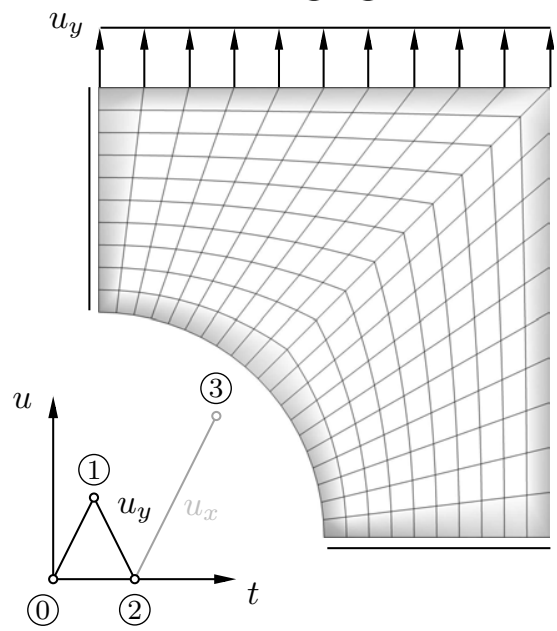

(a)

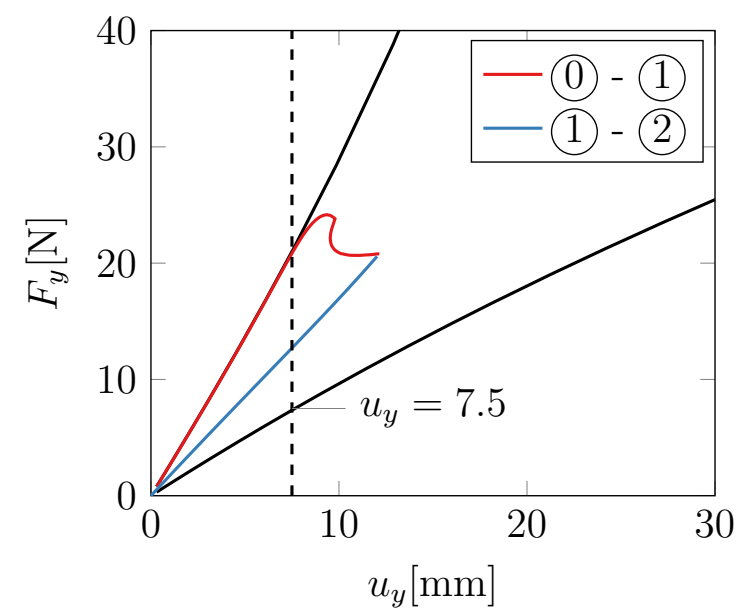

(c)
(2)-(3)

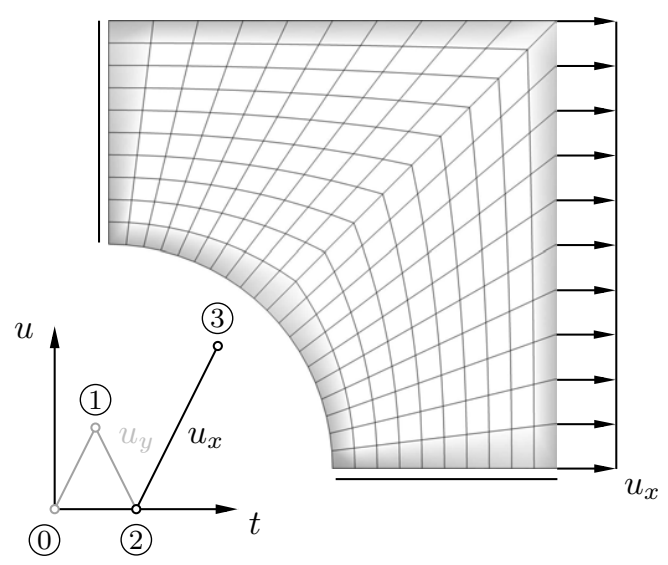

(b)

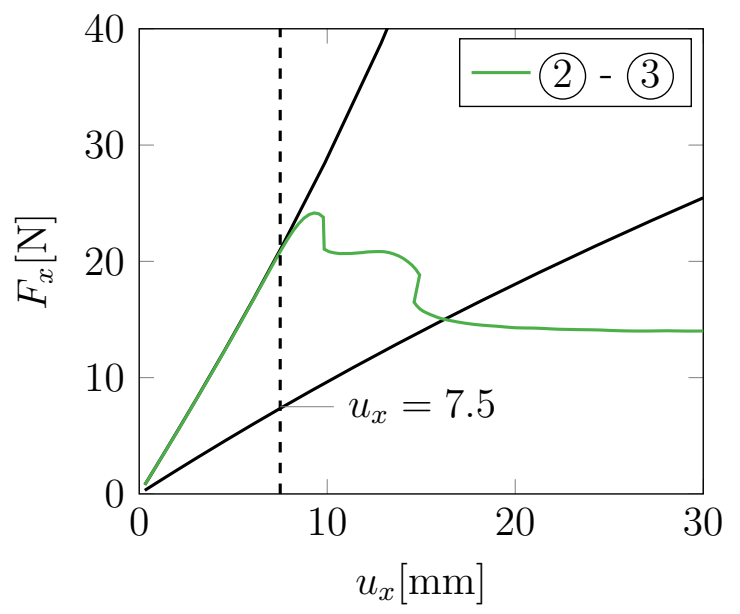

(d)

Figure 4.8: Load-displacement response of a plate with a hole under biaxial loading. (a) History of inhomogeneous Dirichlet boundary conditions on the top of the plate. (b) History of inhomogeneous Dirichlet boundary conditions on the right side of the plate. The solid black curves represent the elastic response of the isotropic neo-Hookean matrix (lower curve) and the overall elastic response of the fibre-reinforced material (upper curve). The dotted lines mark the onset of damage. (c) Load-displacement response along paths (1)-(1) and (1)-(2). (d) Load-displacement response along path (2)-(3).

the Newton iterations for the solution of the algebraic system of equations do not converge.

\section{Plate with a hole under biaxial loading}

A plate as the one depicted in Figure $4.4 \mathrm{a}$ is subjected to a biaxial loading. In a first stage the plate is clamped at the bottom face and stretched along the $Y$-direction by the imposition of Dirichlet boundary conditions at the top face (path (0)-(1) in Figure 4.8a). Once the displacement at the top face reaches a value of $u_{2}=12 \mathrm{~mm}$ the plate is completely unloaded (path (1)-(2) in Figure 4.8a). In a second stage the plate is clamped at the left face and stretched along the $X$-direction by the imposition of Dirichlet boundary conditions 


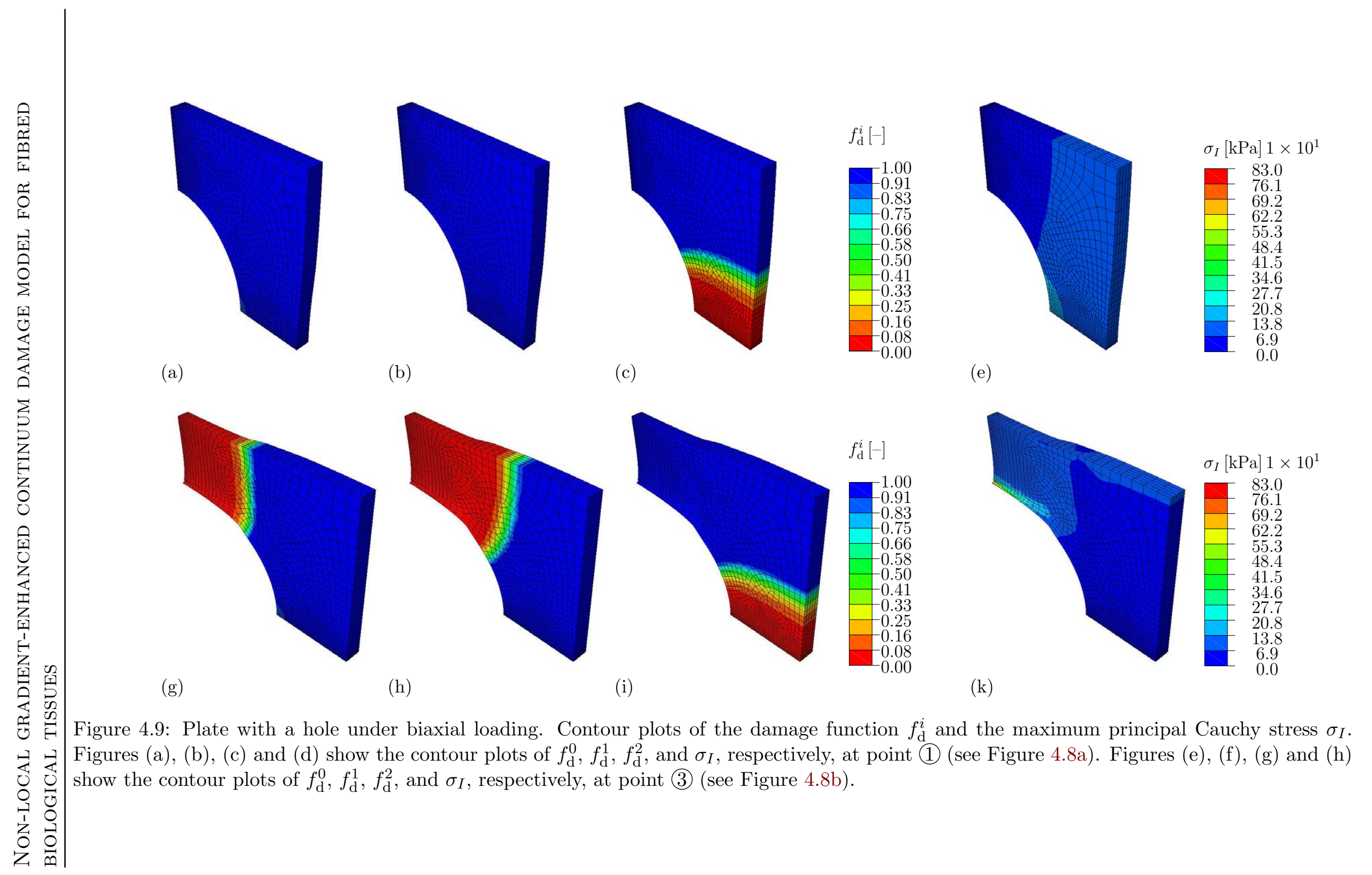


at the right face (path (2)-(3) in Figure 4.8a). The plate is stretched until a maximum displacement of $u_{1}=30 \mathrm{~mm}$ in the right face is reached.

For this problem the same symmetries defined in the previous example hold, hence only $1 / 8$ of the geometry of the plate is discretised. Nevertheless, since damage evolution is expected to be triggered for both families of fibres as well as for the matrix, a non-structured mesh with a finer discretisation around the bottom and left faces is used. A total of 7330 elements is obtained as a result of the aforementioned discretisation. Regarding the solution strategy, the arc-length method is used in order to capture the snap-back that occurs in the loaddisplacement response in paths (1)-(1) and (2)-(3). For the unloading of the plate in path (1)-(2) a standard Newton scheme is used. The material parameters are the same as in the previous example (see Table 4.5). These have been chosen to cause the onset of damage evolution to be triggered first in the fibre reinforcements and later on in the matrix.

In the previous case of the uniaxially loaded plate, damage in the first family of fibres was never activated; this boundary value problem, on the other hand, completely illustrates one of the main features of the proposed model, i.e. the independent evolution of damage in each of the constituents of the composite material. Figure 4.8c shows the load-displacement response of the plate in the first loading and unloading cycle, paths (0)-(1) and (1)-(2) respectively. Here we allow the imposed displacement to reach a maximum value of $u_{y}=12 \mathrm{~mm}$, so that we only capture a first snap-back associated to the damage in the second fibre family while the matrix remains undamaged, i.e., behaves elastically. After the maximum displacement is reached, the plate is completely unloaded and then loaded once again in the $X$-direction. Figure 4.8d shows the load-displacement response of the plate in the last loading path (2)-(3). There, we can appreciate the same characteristic behaviour that was previously observed in the uniaxial stretching of the plate (see Figure 4.5). We have a first snap-back that occurs as a consequence of failure in the first fibre family, followed by a second snap back associated to the onset of damage in the matrix.

Finally, the contour plots of the damage function $f_{\mathrm{d}}^{i}$ are shown in Figure 4.9. In the first row (Figures 4.9a,4.9b, and4.9c), we see the contour plots of the damage function at point (1), i.e. when the plate is stretched along the $Y$-direction. For this case no damage evolution is appreciated in the phases 0 and 1, namely, the neo-Hookean matrix and the first family of fibres. Later on, in the second row (Figures 4.9d,4.9e, and 4.9f), we see the contour plots of the damage function at point (3), i.e. when the plate has reached the maximum imposed displacement, $u_{x}=30[\mathrm{~mm}]$ along the $X$-direction. In these plots we can appreciate that damage has evolved in all the phases: in the neo-Hookean matrix, and also in both fibre families. It is interesting to note that for both loading histories (1)-(1) and (2)-(3), the onset of damage is triggered exactly at the same point, i.e. when the displacements reach a value of $u_{y} \approx 7.5[\mathrm{~mm}]$ and $u_{x} \approx 7.5[\mathrm{~mm}]$ respectively (see Figures $4.8 \mathrm{c}$ and $4.8 \mathrm{~d}$ ). This could be easily explained by the fact that no damage evolution in the neo-Hookean matrix is 
obtained after loading along the path (1)-(2) (see Figure 4.9). Hence, when the plate is loaded along the $X$-direction following the path (2)-(3), the structural response in Figure $4.8 \mathrm{~d}$ would exactly match the response in Figure $4.8 \mathrm{c}$ up to point (1), the point closest to the onset of matrix damage.

\subsection{Summary}

In this chapter a non-local gradient-enhanced continuum damage formulation at large deformations is presented. The model is built around the enhancement of the free energy function of a hyperelastic potential by means of a term that contains the referential gradient of the non-local damage variable. The inclusion of this term ensures an implicit regularisation of the finite element implementation, hence the localisation of damage is no longer controlled by the mesh resolution, but rather by the regularisation parameter $c_{\mathrm{d}}^{i}$, ensuring mesh-objective results of the simulations. In this regard, the proposed model represents an improvement with respect to standard local continuum damage formulations, which require an explicit regularisation to be implemented in order to ensure mesh-objective results.

The elastic constitutive behavior of the material is defined by a hyperelastic functional made of a volumetric contribution and an isochoric contribution. For the volumetric contribution, a quadratic form that serves to enforce the incompressibility constraint $J-1=0$ is used. The isochoric contribution is further subdivided into three contributions associated to three different phases $i=\{0,1,2\}$. Phase 0 is represented by an incompressible neo-Hookean material; phases 1 and 2 are represented by an exponential format that accounts for the stretching along two preferred anisotropy directions, i.e. two fibre families. A $1-d^{i}$-type damage function, i.e. $f_{\mathrm{d}}^{i}=1-d^{i}=\exp \left(\eta_{\mathrm{d}}\left[\kappa_{\mathrm{d}}^{i}-\kappa^{i}\right]\right)$, is also introduced to reproduce the loss of stiffness in each phase $i$.

The Euler-Lagrange equations that govern the boundary value problem are solved in their weak form by means of the minimisation of the total potential energy. The weak form, in turn, serves as a basis for the finite element implementation of the non-local gradientenhanced continuum damage model. To this end we developed an 8-noded Q1P0 hexahedral element following a multivariational approach, which ensures the fulfillment of the incompressibility condition. This element is implemented in Abaqus by means of the user subroutine UEL.

The main capabilities of the proposed model are highlighted with two boundary value problems: a homogeneous state under uniaxial tension, and an anisotropic plate with a hole. The anisotropic plate with a hole, is studied in two parts. In a first stage, where the plate is uniaxially loaded, the mesh-objectivity and the effect of the regularisation parameters on the model are studied. On the one hand, the load-displacement curves in Figure $4.4 \mathrm{~b}$ show that the structural response is the same for three different meshes of 
2548, 4096, and 7700 elements, and the contour plots in Figure 4.6 show no appreciable differences in the distribution of the damage function. From these results it is concluded that the implicit regularisation induced by the gradient-enhancement of the strain energy function guarantees mesh-objective results of the finite element implementation. On the other hand, Figures 4.5 and 4.7 show the effect of the regularisation parameter $c_{\mathrm{d}}^{2}$ in the load-displacement curves and in the distribution of the damage function $f_{\mathrm{d}}^{2}$, respectively. From Figures 4.5 we see that larger values of regularisation lead to higher dissipation, while from Figures 4.7 we see that larger values of regularisation lead to more homogenised solutions of the non-local damage variable, and naturally to a more homogenised damage distribution. In a second stage, the plate is loaded biaxially to highlight the capabilities of the model to capture the evolution of damage in each phase individually, as appreciated in the contour plots of the damage function in Figure 4.9. 
Non-LOCAL GRADIENT-ENHANCED CONTINUUM DAMAGE MODEL FOR FIBRED BIOLOGICAL TISSUES 


\section{Clinical applications}

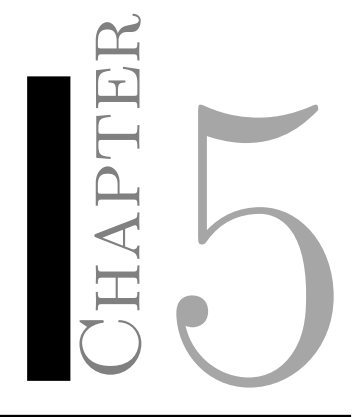

In this chapter we explore two boundary value problems related to biomechanics of the cardiovascular system. Specifically we here study the effect that damage evolution has on the structural response of the artery using the finite element implementation of the gradient-enhanced damage model presented in Chapter 4. Special attention is devoted to the enforcement of residual stresses.

In the first example related to the biomechanics of arteries, we study the evolution of damage in an idealised geometrical configuration that resembles a balloon angioplasty. In this case only a cylindrical sector (wedge) of the complete geometry is discretised, a simplification that poses some restrictions on the boundary conditions, loads, and structural parameters that can be defined. In the second and final example, a full-3D artery-like tube under internal pressure illustrates a more general configuration where no particular restrictions on the boundary conditions, loads, or material parameters must be taken into consideration.

\subsection{Residual stresses in arteries}

The incorporation of residual stresses is of key importance within the modelling and simulation of soft biological tissues, i.e. arteries. Different concepts have been discussed in the literature to account for these equilibrated stress contributions present in the absence of external loading. The procedure employed here to incorporate such effects is based on a multiplicative composition of the total deformation gradient as discussed by Holzapfel et al. [72]. This procedure aims to serve as a basis to reproduce the opening angle experiment (See Chuong and Fung [16]), which shows that excised in-vitro artery samples spring open after a cut in the radial direction. The mathematical framework developed to study the opening angle experiment relies on the assumption that arteries can be regarded as perfect cylindrical geometries. Hence, such a simplification allows to use the same framework for 


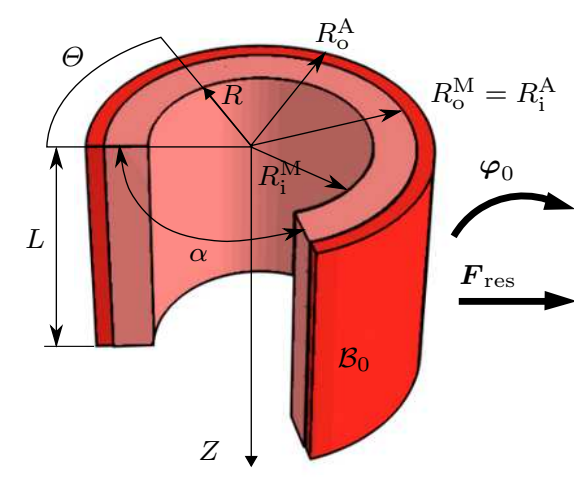

(a)

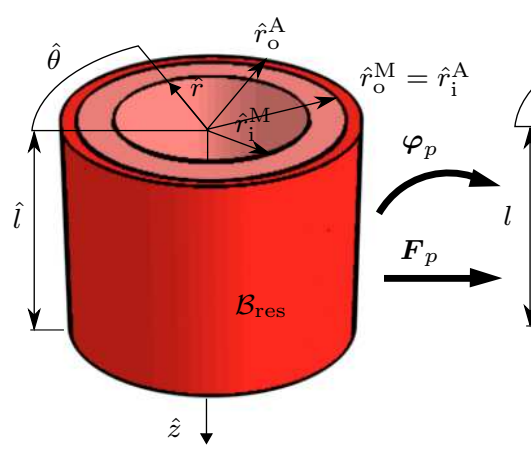

(b)

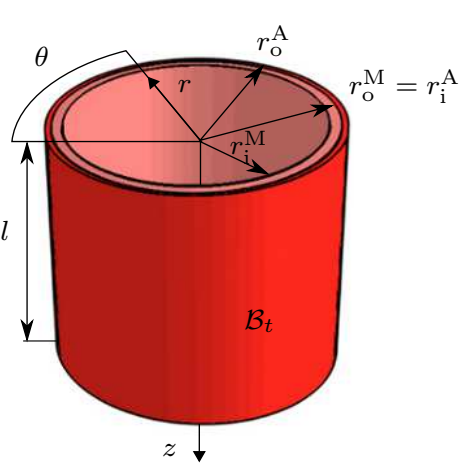

(c)

Figure 5.1: Bending and inflation of a double-layered thick-walled cylindrical tube, cf. Holzapfel et al. [72]: (a) A stress-free (referential) configuration $\mathcal{B}_{0}$. Base vectors $\boldsymbol{E}_{r}, \boldsymbol{E}_{\theta}, \boldsymbol{E}_{z}$. (b) A residually stressed but load-free configuration $\mathcal{B}_{\text {res }}$. Base vectors $\hat{\boldsymbol{e}}_{r}, \hat{\boldsymbol{e}}_{\theta}, \hat{\boldsymbol{e}}_{z}$. (c) A residually stressed and loaded (current) configuration $\mathcal{B}_{t}$. Base vectors $\boldsymbol{e}_{r}, \boldsymbol{e}_{\theta}, \boldsymbol{e}_{z}$. (Adopted from Waffenschmidt and Menzel [118] with kind permission of Elsevier.)

any type of blood vessel. To be specific, we consider three different configurations that could be interpreted as the inverse sequence of the different stages in the opening angle experiment, cf. Figure 5.1. a) First we consider a stress-free reference configuration $\mathcal{B}_{0}$ that represents the open sector of the artery. b) Second we consider a load-free residually stressed configuration $\mathcal{B}_{\text {res }}$ that represents the excised in-vitro sample of the artery before cutting it radially. c) Finally, we consider a current configuration $\mathcal{B}_{t}$ that represents the in-vivo artery, i.e. the artery under the effect of internal blood pressure. As usual, coordinates in the referential and current configuration are expressed in upper and lower case characters, respectively. Coordinates in the intermediate configuration $\mathcal{B}_{\text {res }}$ are expressed in lower case characters with a hat, to indicate that, although it corresponds to a deformed configuration, this is not the final deformed configuration that results from the application of internal blood pressure in the artery.

We now introduce a deformation gradient-type tensor $\boldsymbol{F}_{\text {res }}$ which transforms line elements from the stress-free reference configuration $\mathcal{B}_{0}$ to the residually stressed configuration $\mathcal{B}_{\text {res }}$ For a perfectly cylindrical geometry, $\boldsymbol{F}_{\text {res }}$ can be expressed in terms of the principal stretches in a cylindrical coordinate system, as

$$
\boldsymbol{F}_{\mathrm{res}}=\left.\lambda_{i} \hat{\boldsymbol{e}}_{i} \otimes \boldsymbol{E}_{i}\right|_{i \in\{r, \theta, z\}}
$$

The radial and the circumferential principal stretches can be expressed as $\lambda_{r}=R /\left[\hat{r} k \lambda_{z}\right]$ and $\lambda_{\theta}=k \hat{r} / R$, where $R=\sqrt{R_{\mathrm{i}}^{2}+k \lambda_{z}\left[\hat{r}^{2}-\hat{r}_{\mathrm{i}}^{2}\right]}$. In these relations, we prescribe the inner radius of the closed configuration $\hat{r}_{\mathrm{i}}$, the inner radius of the opened configuration $R_{\mathrm{i}}$, the opening angle $\alpha$, and the axial residual stretch $\lambda_{z}$, and introduce the opening angle parameter as $k=2 \pi /[2 \pi-\alpha]$. Finally, the resulting deformation gradient tensor relating 
0. Given: $\hat{r}_{\mathrm{i}}, R_{\mathrm{i}}, \alpha$, and $\lambda_{z}$ (See Figure 5.1)

1. Compute opening angle parameter $k=2 \pi /[2 \pi-\alpha]$

2. Determine physical Cartesian coordinates $(x, y, z)$ of the Gauss-points for the closed configuration and convert these into cylindrical coordinates $(r, \Theta, z)$

3. Compute Cartesian base vectors for the closed configuration

$$
\begin{aligned}
& \hat{\boldsymbol{e}}_{x}=\cos (\Theta) \hat{\boldsymbol{e}}_{r}+\sin (\Theta) \hat{\boldsymbol{e}}_{\Theta} \\
& \hat{\boldsymbol{e}}_{y}=-\sin (\Theta) \hat{\boldsymbol{e}}_{r}+\cos (\Theta) \hat{\boldsymbol{e}}_{\Theta} \\
& \hat{\boldsymbol{e}}_{z}=\hat{\boldsymbol{e}}_{x} \times \hat{\boldsymbol{e}}_{y}
\end{aligned}
$$

4. Compute cylindrical coordinates $(R, \Theta, Z)$ for the opened configuration

$$
\begin{aligned}
R & =\sqrt{k \lambda_{z}\left[\hat{r}^{2}-\hat{r}_{\mathrm{i}}^{2}\right]+R_{\mathrm{i}}^{2}} \\
\Theta & =\hat{\Theta} / k \\
Z & =\hat{z} / \lambda_{z}
\end{aligned}
$$

5. Compute Cartesian base vectors for the opened configuration

$$
\begin{aligned}
& \boldsymbol{E}_{X}=\cos (\Theta) \boldsymbol{E}_{R}+\sin (\Theta) \boldsymbol{E}_{\Theta} \\
& \boldsymbol{E}_{Y}=-\sin (\Theta) \boldsymbol{E}_{R}+\cos (\Theta) \boldsymbol{E}_{\Theta} \\
& \boldsymbol{E}_{Z}=\boldsymbol{E}_{X} \times \boldsymbol{E}_{Y}
\end{aligned}
$$

6. Compute radial and circumferential stretches

$$
\begin{aligned}
& \lambda_{r}=R /\left[\hat{r} k \lambda_{z}\right] \\
& \lambda_{\Theta}=\hat{r} k / R
\end{aligned}
$$

7. Compute initial deformation gradient at each Gauss-point in Cartesian coordinates $\boldsymbol{F}_{\mathrm{res}}=\lambda_{r} \hat{\boldsymbol{e}}_{x} \otimes \boldsymbol{E}_{X}+\lambda_{\Theta} \hat{\boldsymbol{e}}_{y} \otimes \boldsymbol{E}_{Y}+\lambda_{z} \hat{\boldsymbol{e}}_{z} \otimes \boldsymbol{E}_{Z}$

Table 5.1: Flowchart for the computation of the initial residual strain field in a cylindrical FEmesh

the referential and the current configuration can then be written as

$$
\boldsymbol{F}=\boldsymbol{F}_{p} \cdot \boldsymbol{F}_{\text {res }}
$$

Composition (5.2) can conveniently be used to model residual stresses within finite element formulations for the simulation of tube-like boundary value problems.

\subsection{Finite element modelling of damage in balloon angioplasty}

In order to show the computational performance and robustness of the proposed non-local gradient-enhanced damage model within the context of biomechanics, we now consider the finite element simulation of a balloon angioplasty procedure. Balloon angioplasty is a common surgical intervention used to extend or reopen narrowed blood vessels in order to 


\begin{tabular}{|c|c|c|c|c|c|c|c|}
\hline Type & & Symbol & Balloon & Intima & Media & Adventitia & Unit \\
\hline \multirow{3}{*}{ Geometry } & & $R_{\mathrm{i}}$ & 0.75 & 1.35 & 1.43 & 1.92 & {$[\mathrm{~mm}]$} \\
\hline & & $H$ & 0.1 & 0.08 & 0.49 & 0.33 & {$[\mathrm{~mm}]$} \\
\hline & & $L$ & 5.0 & 7.5 & 7.5 & 7.5 & {$[\mathrm{~mm}]$} \\
\hline \multirow{4}{*}{ Structure } & & $\beta^{1}$ & 90.0 & - & +21.700 & +62.260 & [deg] \\
\hline & & $\beta^{2}$ & 0.0 & - & -21.700 & -62.260 & [deg] \\
\hline & & $\varkappa$ & 0.0 & - & 0.19 & 0.036 & {$[-]$} \\
\hline & & $\alpha$ & - & - & 45.0 & 90.0 & [deg] \\
\hline \multirow{6}{*}{ Elasticity } & \multirow{2}{*}{ Matrix } & $\mu_{\mathrm{e}}$ & 1000.0 & 0.27 & 27.0 & 2.7 & {$[\mathrm{kPa}]$} \\
\hline & & $\kappa_{\mathrm{e}}$ & $100.0 \mu_{\mathrm{e}}^{\mathrm{B}}$ & $100.0 \mu_{\mathrm{e}}^{\mathrm{I}}$ & $100.0 \mu_{\mathrm{e}}^{\mathrm{M}}$ & $100.0 \mu_{\mathrm{e}}^{\mathrm{A}}$ & {$[\mathrm{kPa}]$} \\
\hline & \multirow{4}{*}{ Fibres } & $k_{1}^{1}$ & 500000.0 & - & 0.64 & 5.1 & {$[\mathrm{kPa}]$} \\
\hline & & $k_{2}^{1}$ & 0.001 & - & 3.54 & 15.4 & {$[-]$} \\
\hline & & $k_{1}^{2}$ & 1.0 & - & 0.64 & 5.1 & {$[\mathrm{kPa}]$} \\
\hline & & $k_{2}^{2}$ & 0.55 & - & 3.54 & 15.4 & {$[-]$} \\
\hline \multirow{4}{*}{ Damage } & \multirow{2}{*}{ Matrix } & $\eta_{\mathrm{d}}^{0}$ & - & - & \multirow{2}{*}{\multicolumn{2}{|c|}{$\begin{array}{l}\text { variable } \\
\text { variable }\end{array}$}} & {$\left[\mathrm{kPa}^{-1}\right]$} \\
\hline & & $\kappa_{\mathrm{d}}^{0}$ & - & - & & & {$[\mathrm{kPa}]$} \\
\hline & \multirow{2}{*}{ Fibres $(i=1,2)$} & $\eta_{\mathrm{d}}^{i}$ & - & - & \multirow{2}{*}{\multicolumn{2}{|c|}{$\begin{array}{l}\text { variable } \\
\text { variable }\end{array}$}} & {$\left[\mathrm{kPa}^{-1}\right]$} \\
\hline & & $\kappa_{\mathrm{d}}^{i}$ & - & - & & & {$[\mathrm{kPa}]$} \\
\hline \multirow{4}{*}{ Regularisation } & \multirow{2}{*}{ Matrix } & $c_{\mathrm{d}}^{0}$ & - & - & \multicolumn{2}{|c|}{1.0} & {$\left[\mathrm{kPa}^{-1} \mathrm{~mm}^{2}\right]$} \\
\hline & & $\beta_{\mathrm{d}}^{0}$ & - & - & \multicolumn{2}{|c|}{1000.0} & {$\left[\mathrm{kPa}^{-1}\right]$} \\
\hline & \multirow{2}{*}{ Fibres $(i=1,2)$} & $c_{\mathrm{d}}^{i}$ & - & - & \multicolumn{2}{|c|}{1.0} & {$\left[\mathrm{kPa}^{-1} \mathrm{~mm}^{2}\right]$} \\
\hline & & $\beta_{\mathrm{d}}^{i}$ & - & - & \multicolumn{2}{|c|}{1000.0} & {$\left[\mathrm{kPa}^{-1}\right]$} \\
\hline
\end{tabular}

Table 5.2: Set of geometrical, structural and material parameters used in the simulation of the balloon angioplasty. The material parameters are subdivided in elastic- and damage-related parameters associated with the matrix and the fibre-response. A set of upper indices B, I, M, and A is used to differentiate the parameters for the balloon, the intima, the media, and the adventitia respectively. Parameters $\beta^{1}$ and $\beta^{2}$ measure the angle of material vectors $\boldsymbol{a}_{0}^{1}$ and $\boldsymbol{a}_{0}^{2}$ with respect to circumferential direction $\boldsymbol{e}_{\theta}$.

restore the continuous blood flow in, for instance, atherosclerotic arteries. A balloon, typically made of a highly flexible rubber-like plastic sheet, is inserted within the blocked artery and inflated by means of high pressure using magnitudes between $p=600.0-2000.0[\mathrm{kPa}]$, or $p=6.0-20.0$ [bar]. The balloon contacts the inside of the artery and slightly overstretches the arterial tissue beyond the physiological pressure level. Inelastic, i.e. predominantly damage-related and elastoplastic processes are induced during its inflation which result in an irreversible deformation within the artery upon this supraphysiological loading. As a beneficial consequence, provided that the inelastic deformations do not exceed a specific limit, higher deformations can be obtained within the same pressure level and a continuous blood flow can be guaranteed. 


\begin{tabular}{ccccccccccccccc}
\hline & Set 1 & Set 2 & Set 3 & Set 4 & Set 5 & Set 6 & Set 7 & Set 8 & Set 9 & Set 10 & Set 11 & Set 12 & Set 13 & Set 14 \\
\hline$\eta_{\mathrm{d}}^{0}$ & 1.0 & 1.0 & 1.0 & - & - & - & 1.0 & 8.0 & 9.0 & 11.0 & 9.0 & 9.0 & - & 9.0 \\
$\kappa_{\mathrm{d}}^{0}$ & 1.0 & 4.0 & 8.0 & - & - & - & 8.0 & 8.0 & 12.0 & 8.0 & 10.0 & 20.0 & - & 12.0 \\
$c_{\mathrm{d}}^{0}$ & 5.0 & 5.0 & 5.0 & - & - & - & 12.5 & 5.0 & 5.0 & 5.0 & 5.0 & 5.0 & - & 5.0 \\
\hline$\eta_{\mathrm{d}}^{1,2}$ & - & - & - & 7.5 & 5.0 & 1.5 & 1.0 & 1.5 & 3.0 & 3.0 & 3.0 & 3.0 & 3.0 & 3.0 \\
$\kappa_{\mathrm{d}}^{1,2}$ & - & - & - & 1.0 & 1.0 & 2.0 & 2.0 & 2.0 & 2.0 & 2.0 & 2.0 & 2.0 & 1.0 & 1.0 \\
$c_{\mathrm{d}}^{1,2}$ & - & - & - & 5.0 & 5.0 & 5.0 & 3.0 & 5.0 & 5.0 & 5.0 & 5.0 & 5.0 & 5.0 & 5.0 \\
\hline
\end{tabular}

Table 5.3: Sets of material parameters used to study the balloon angioplasty (Sets 1-12) and the inflation of a full 3D artery-like tube (Sets 13-14). Results of the simulation for the balloon angioplasty are shown in Figures 5.7 - 5.11. Results of the simulation for the artery-like tube are shown in Figures 5.12 - 5.15. 
The physical modelling and numerical simulation of complete surgical interventional procedures has increasingly drawn much attention during the last decade. In this regard, balloon angioplasty is certainly one of the most important surgical intervention techniques. Several studies on the modelling and simulation on the mechanics of balloon angioplasty exist in the literature. In order to enable reliable predictions regarding, for instance, the stress distribution or damage-related softening effects in patient-specific atherosclerotic arteries subjected to angioplasty, sophisticated constitutive models for the individual components are indispensable. While several convincing material models allowing for an elastic anisotropic response at finite deformation have already been established, there is still need for physically sound and numerically robust and efficient models that take the inelastic response of the structure into account. Especially regarding the aforementioned angioplasty procedure, there are several contributions that consider the associated mechanisms as elastoplastic while only few take typical damage-related effects into account.

Within the contributions by Holzapfel et al. [119, 120] experimental methods such as magnetic resonance imaging are used to obtain layer- and component-specific geometrical and histological data of a human iliac artery with an eccentric stenosis. Furthermore, the mechanics of the angioplasty are investigated from a computational point of view by performing a finite element study on the identified atherosclerotic arterial geometry by means of an inelastic anisotropic constitutive model introduced by Holzapfel et al. [121]. Herein, the elastic response is governed by the anisotropic constitutive model proposed by Holzapfel et al. [72] while the fiber-reinforced composite is assumed to show elastoplastic behaviour and, essentially following Miehe [122], is based on a multiplicative split of the deformation gradient and the concept of multisurface plasticity including two slip-systems for the two collagen fibre families. In a later work, Gasser and Holzapfel [123] applied the same model to the angioplasty by considering the balloon-induced overstretch of remnant non-diseased tissues in atherosclerotic arteries where a stenotic residually stressed artery composed of adventitia, media and a model plaque was investigated during contact interaction with the balloon catheter. Kiousis et al. [124] experimentally investigated the mechanical characteristics of the transient expansion of six commercially available balloon-expandable stent systems and developed a finite element model based on these experimental results.

In contrast to the aforementioned studies which predominantly considered the balloon angioplasty process to be characterised by elastoplastic behaviour, Alastrué et al. [125] used a continuum damage model that accounts for separate damage contributions for the matrix and the fibers, see also Alastrué [126] for more details. This model was proposed by Calvo et al. [127] where matrix and fibre damage effects are described by the same approach. A multitude of other damage and failure formulations has been published, such as the article by Volokh [128], or the recent publication by Balzani et al. [129]. However, to the authors' knowledge, almost all of these works consider damage as a purely local 


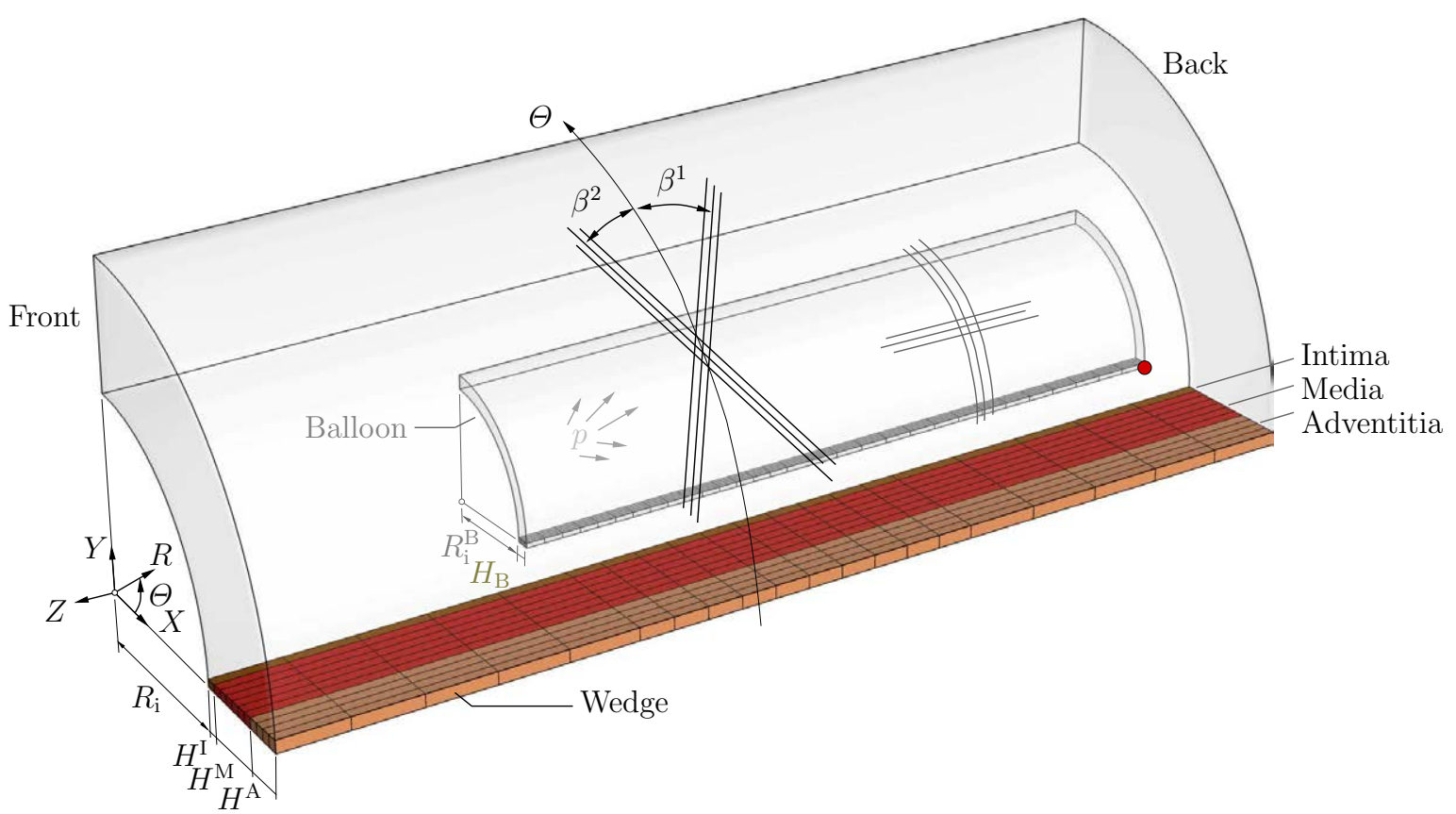

Figure 5.2: Model of the balloon angioplasty. Initial setup including the balloon (grey) and the artery consisting of three layers, i.e. intima (dark red), media (red), adventitia (orange). Balloon: the nodes at the front left edge are fixed in radial $R$-direction, all nodes are fixed in circumferential $\Theta$-direction while the nodes at the back face are fixed in axial $Z$-direction. Artery: all nodes are fixed in circumferential $\Theta$-direction while the nodes at the front and the back faces are fixed in axial $Z$-direction. An internal pressure $p$ is applied to the inner radius of the balloon. The model is idealised as a configuration of two concentric hollow cylinders. A cylindrical sector, i.e. a wedge, of 1.125 [deg] is used for the finite element representation.

phenomenon without robust regularisation techniques involved, which, in view of finite element applications, may result in highly mesh-dependent and physically questionable simulations. So far, a fully non-local gradient-extended damage formulation allowing for large deformations and an individual damage evolution for matrix and fibres with special regard to anisotropic biological tissues has not yet been established.

In what follows is a set of upper indices B, I, M, and A which will be used to differentiate among the parameters associated with the balloon, the intima, the media, and the adventitia respectively.

\subsubsection{Setup of the boundary value problem}

The simulation of damage effects associated with the balloon angioplasty is fairly complex due to the complex geometrical configuration of the actual blood vessels. Hence, in order to be able to develop a first approximation that qualitatively represents the original set up of the balloon angioplasty, we assume an idealised configuration of two concentric hollow cylinders representing the balloon and the artery respectively. A cylindrical sector of this idealised configuration, i.e. a wedge, is used for the finite element representation (See Figure 
5.2). This is, of course, a drastic simplification of the real physical set up, but conveniently enables us to use the aforementioned procedure discussed in Section 5.1 in order to include the residual stresses within the artery. Furthermore, this simplifies the interpretation of the results, as geometric inhomogeneities do not contribute to the mechanical response.

The wedge model represents a cylindrical sector of 2.25 [deg] from the complete geometry that defines the set up of the balloon angioplasty. The particular dimensions are summarised in Figure 5.2 and Table 5.2. The artery has a layered composite-type structure and is subdivided into intima $(1 \times 20$ elements $)$, media $(6 \times 20$ elements $)$ and adventitia ( $4 \times 20$ elements). The balloon is discretised by $4 \times 30$ elements, so that the whole boundary value problem consists of 340 elements. The media and adventitia of the artery are discretised by the gradient-enhanced finite element formulation via the UEL-interface, whereas the intima and the balloon are discretised by Abaqus-based eight-noded hybrid hexahedral finite elements of C3D8H-type. The reason for this modelling approach is that the embedded Abaqus contact formulation does not accept purely UEL-based finite elements. Therefore, the elements associated to contact are modelled with original Abaqus elements. For the balloon, we assume a hyperelastic nearly incompressible orthotropic material behaviour according to Gasser et al. [7]. The two fibre families are aligned with respect to the axial and circumferential directions and have no dispersion in their mean orientation, i.e. $\varkappa^{\mathrm{B}}=0.0$, see Figure 5.2. The material parameters are chosen so that the fibres oriented in circumferential direction are fairly soft at small strains but rapidly stiffen at larger strains. The fibres oriented in axial direction are chosen to be already stiff from the beginning of the deformation. For general aspects of the mechanics and thermodynamics of rubber and rubber balloons, we refer the reader to the article by Müller and Struchtrup [130] or the book by Müller and Strehlow [131].

For the intima, an isotropic nearly incompressible neo-Hooke material is used, which is assumed to be very soft and thus almost negligible compared to the media and adventitia. This, on the one hand, resembles the marginal contribution of a healthy intima on the mechanical response. On the other hand, the incorporation of the intima via Abaqus elements enables us to use the embedded Abaqus contact formulation. As a limitation of this work, one might argue that in certain cases the intima cannot be neglected within an atherosclerotic arterial wall possibly affected by pathological plaques.

The media and adventitia are modelled by the gradient-enhanced damage formulation accounting for damage effects in the matrix and in each fibre family individually. Both layers are characterised by individual material and structural parameters, see Table 5.2. The material parameters are adopted from Holzapfel and Ogden [132, p. 55], where the shear modulus of the nearly incompressible neo-Hookean ground substance of the media is assumed to be ten times stiffer than the adventitia. To enforce quasi-incompressibility, the bulk moduli in the media and the adventitia, $\kappa_{\mathrm{e}}^{\mathrm{M}}$ and $\kappa_{\mathrm{e}}^{\mathrm{A}}$, are chosen to be 100 times larger 
than the related shear moduli, $\mu_{\mathrm{e}}^{\mathrm{M}}$ and $\mu_{\mathrm{e}}^{\mathrm{A}}$, respectively. In contrast to Waffenschmidt et al. [110], we note that we here make use of a quasi-incompressible neo-Hookean format based on an isochoric-volumetric split.

The structural parameters are adopted from Alastrué et al. [133] and also specified in Table 5.2. The material fibre orientations in the media and the adventitia are given by $\boldsymbol{a}_{0}^{1,2}=\sin \left(\beta^{1,2}\right) \boldsymbol{e}_{Z} \pm \cos \left(\beta^{1,2}\right) \boldsymbol{e}_{\Theta}$. The mean fibre orientation angles $\beta^{1,2}$ in each layer are specified at the integration point level of each finite element which results in a helical fibre distribution in the artery, see Figure 5.2. Furthermore, small dispersions on the mean orientation of the fibres are included, i.e. $\varkappa \neq 0$ for every layer in the artery. For the media, we choose $\varkappa^{\mathrm{M}}=0.190$, which indicates a dispersed character of the fibres. In the adventitia, the value $\varkappa^{\mathrm{A}}=0.036$ is very close to zero, reflecting an almost transversely isotropic state for each fibre family, see Waffenschmidt et al. [110] for details.

Effects due to residual stresses are also incorporated in the present model following the procedure discussed in Section 5.1. Even though described for a single layered tube, this procedure can be easily extended in an analogous way to create residual strain fields for two or even more layers. Residual stresses are then incorporated by defining $\boldsymbol{F}_{\text {res }}$ at each Gauss-point of the finite-element mesh. This deformation gradient field has to be provided in terms of the underlying coordinate system used by the finite-element code which is typically described by a Cartesian basis. The procedure to compute $\boldsymbol{F}_{\text {res }}$ is summarised in Table 5.1, where the inner radii of the closed and opened configuration $R_{\mathrm{i}}$ and $\hat{r}_{\mathrm{i}}$, the opening angle $\alpha$, and the longitudinal stretch $\lambda_{\hat{z}}$, are given as input parameters. Here, different values of the opening angle are given for the media and the adventitia. It should also be noted that the geometry presented in Figure 5.2 would correspond, in this case, to the intermediate configuration in Figure 5.1. With that clarification at hand, the opening angles and radii are prescribed to values of $R_{\mathrm{i}}^{\mathrm{M}}=1.5[\mathrm{~mm}], R_{\mathrm{i}}^{\mathrm{A}}=2.5[\mathrm{~mm}], \alpha^{\mathrm{M}}=45.0[\mathrm{deg}]$ and $\alpha^{\mathrm{A}}=90.0$ [deg] while an axial residual stretch is neglected at this stage so that $\lambda_{\hat{z}}=1.0$, cp. Table 5.2 or Tobias Waffenschmidt [134] for a more detailed study on the influence of different opening angles and radii.

The boundary conditions for the balloon are specified such that the nodes at the front left edge of the balloon, cf. Figure 5.2, are fixed in radial $R$-direction. All nodes are fixed in circumferential $\Theta$-direction while the nodes at the back face of balloon are fixed in axial $Z$-direction. For the artery, all nodes are fixed in circumferential $\Theta$-direction while the nodes at the front and the back faces of the artery are fixed in axial $Z$-direction. The nonlocal damage field is initialised as $\left.\phi^{i}\right|_{t_{0}}=\kappa_{\mathrm{d}}^{i}$. Furthermore, we apply an internal pressure $p$ to the balloon. We make use of an arc-length method in order to follow the post-peak branch of the load-displacement curves in the damage regime. For this particular solution method the pressure magnitude is not prescribed explicitly but is part of the solution itself; therefore, the arc-length scheme cannot produce a solution at given load or displacement 
values directly. This complicates a cyclic force-driven deformation test. A brief review on the arc-length method in the context of the Abaqus-related UEL-implementation is provided in Waffenschmidt et al. [110].

As indicated above, the usage of the contact formulation embedded in Abaqus in connection with implemented user-elements is not straightforwardly possible. In order to enable the application of the Abaqus contact algorithms, those parts of the problem which are supposed to make contact with each other are discretised by Abaqus-based finite elements, i.e. for the intima and the balloon $\mathrm{C} 3 \mathrm{D} 8 \mathrm{H}$ elements are used. Without going into detail, we define a contact interaction between the outer radius of the balloon (master surface) and the inner radius of the artery (slave surface), see Figure 5.2. Here, we mostly rely on the default contact conditions provided by Abaqus. We define a contact pair of surfaceto-surface-type, neglect friction and use a linear penalty method in order to enforce the contact constraint together with a hard pressure-overclosure relationship without physical softening.

\subsubsection{Results}

The mechanical response of the balloon is represented by the load-displacement diagram in Figure 5.3(c), where the internal pressure $p$ applied to the balloon is plotted against the radial displacement of a node lying at the outer surface of the balloon and at $Z=0[\mathrm{~mm}]$, namely $u_{r_{\mathrm{o}}}^{\mathrm{B}}$ (See the red-marked node in Figure 5.2). We consider three cases: Case 1 , the inflation of the balloon, i.e. no contact with the artery is taken into account; Case 2, the inflation of the balloon including contact with a stress-free artery, i.e. the angioplasty; and Case 3, the inflation of the balloon including contact with a residually stressed artery, i.e. the angioplasty with residual stresses. For all the cases, the balloon shows a degressive stiffness within the range of $p \in[0,150][\mathrm{kPa}]$, approximately. During this first stage fibres have an almost negligible contribution to overall structural stiffness and the load is mainly supported by the neo-Hookean matrix. This degressive stiffness is consistent with the analytical solution for the inflation of a neo-Hookean tube (See Holzapfel [135]). Once $p$ surpasses the critical value of approximately $150[\mathrm{kPa}]$, a progressive stiffening in the load-displacement curve is observed for all the cases. For Case 1 this is explained by the progressive stretching of the fibres in the balloon, especially of those along the circumferential direction. For Cases 2 and 3 the same observation can be made, but we have to add the contribution of the artery once it makes contact with the balloon, which is why stiffening occurs more rapidly for these cases than it does for Case 1. For Case 2 the balloon makes contact with the artery at $u_{r_{0}}^{\mathrm{B}}=0.500[\mathrm{~mm}]$, which is exactly the gap between the balloon and the artery in the initial set up (see Figure 5.2 and values in Table 5.2). For Case 3, on the other hand, the balloon makes contact with the artery 

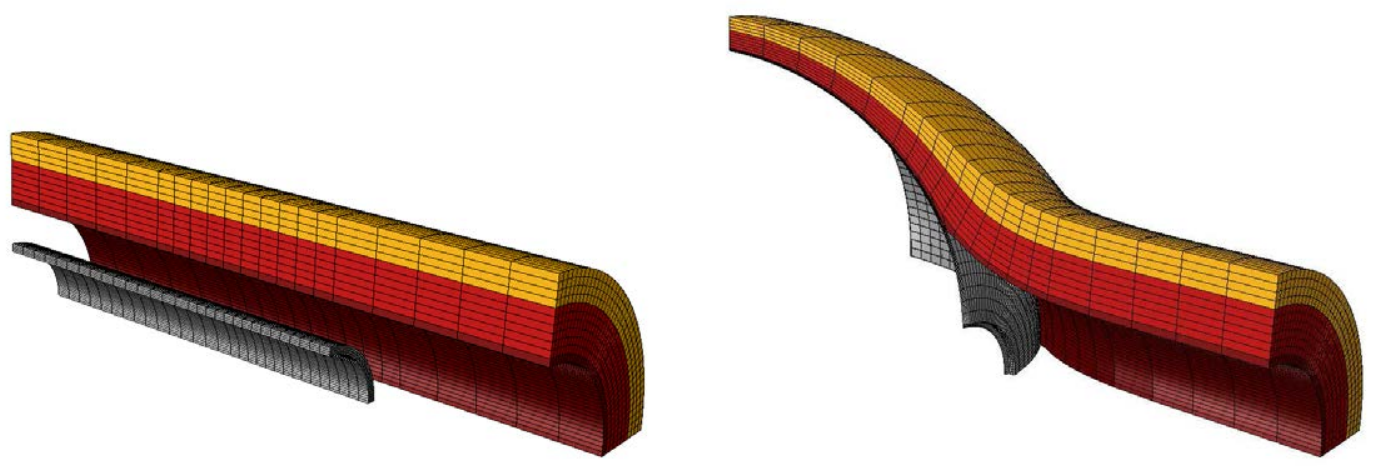

(a)

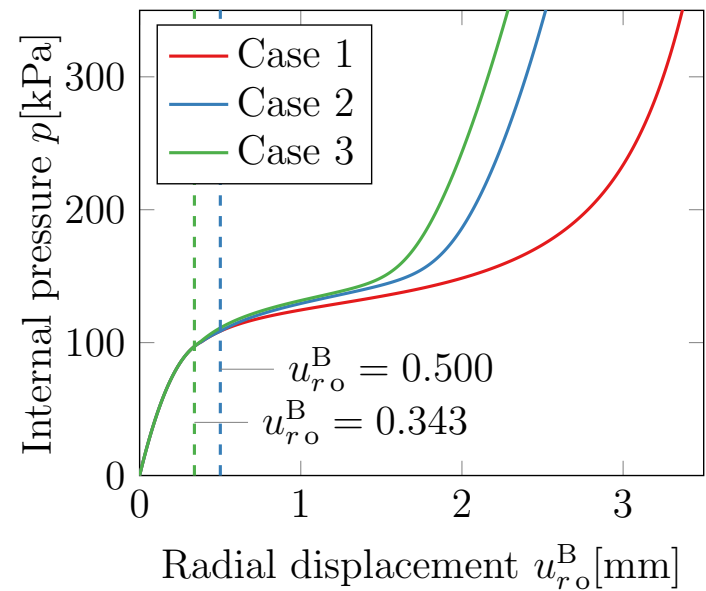

(c) (b)

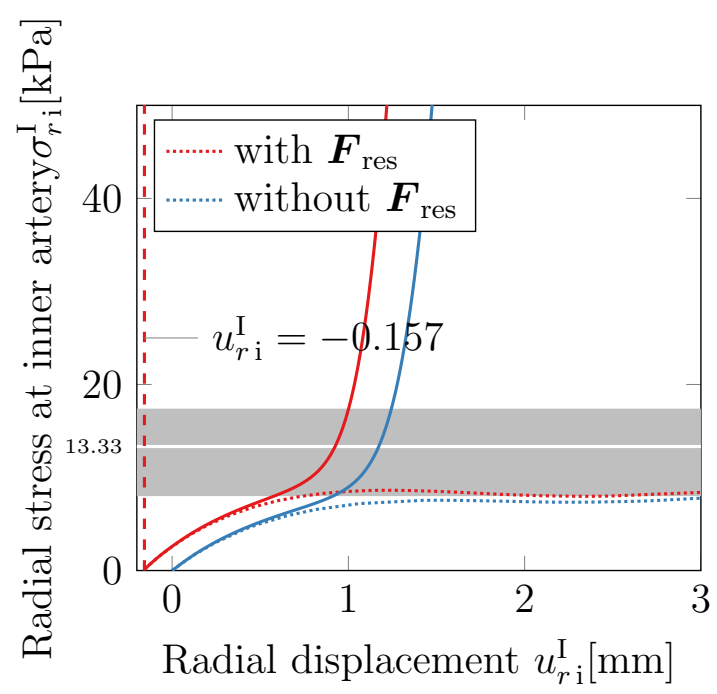

(d)

Figure 5.3: Balloon angioplasty. (a) Initial set up. (b) Characteristic deformation under the specified boundary conditions. (c) Load-displacement-response of the balloon only. Degressive stiffness before contact at $u_{r_{\mathrm{o}}}^{\mathrm{B}}=0.5[\mathrm{~mm}]$ and $u_{r_{\mathrm{o}}}^{\mathrm{B}}=0.343[\mathrm{~mm}]$ for Case 2 and Case 3 respectively. (d) Elastic load-displacement-response of the artery with/without residual stresses. Solid lines represent the response of the fibre-reinforced material, dotted lines represent the response of the neo-Hookean matrix, the grey region represents the physiological blood pressure range, the white line represents the mean physiological blood pressure value.

at $u_{r_{\mathrm{o}}}^{\mathrm{B}}=0.343[\mathrm{~mm}]$. The reason for contact to be initiated at an earlier stage is due to the enforcement of residual stresses in the artery. This effect is discussed in more detail below. The elastic response of the artery during the angioplasty is represented by the load-displacement diagram in Figure 5.3(d). Here, we focus on the events that take place after the balloon makes contact with the artery. The horizontal axis represents the radial displacement of a point located at the inner surface of the artery and at $Z=0[\mathrm{~mm}]$, namely $u_{r \mathrm{i}}^{\mathrm{I}}$, while the vertical axis represents the radial component of the Cauchy stress tensor of a material point at the same location, namely $\sigma_{r \mathrm{i}}^{\mathrm{I}}$. This component of the stress tensor 


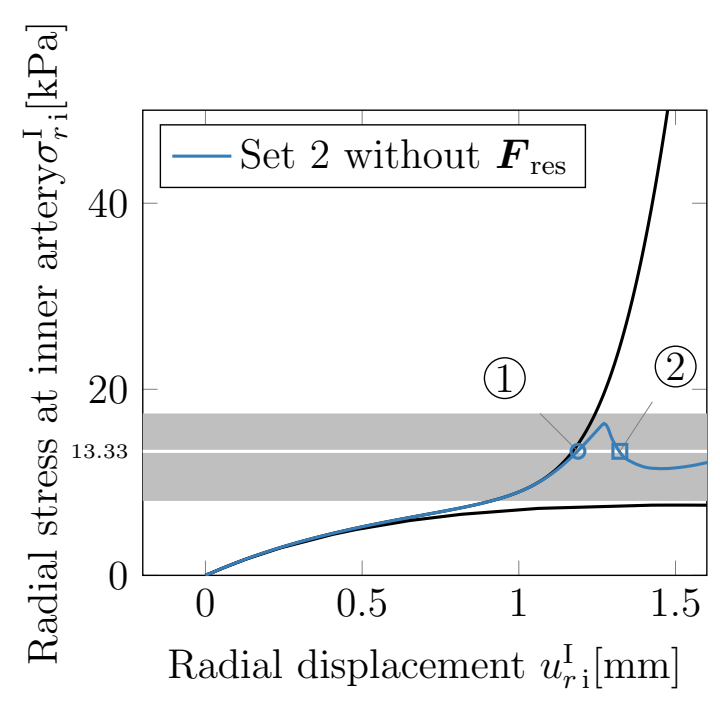

(a)

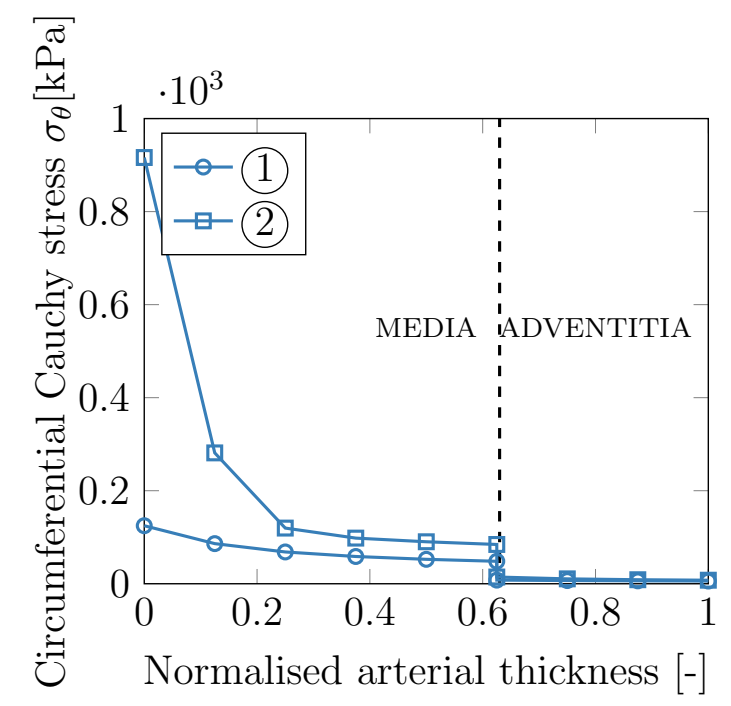

(c)

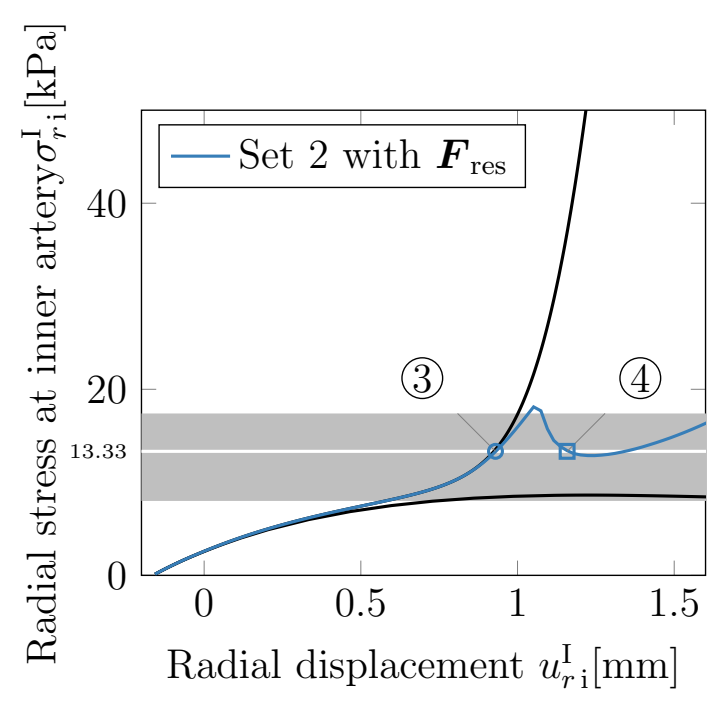

(b)

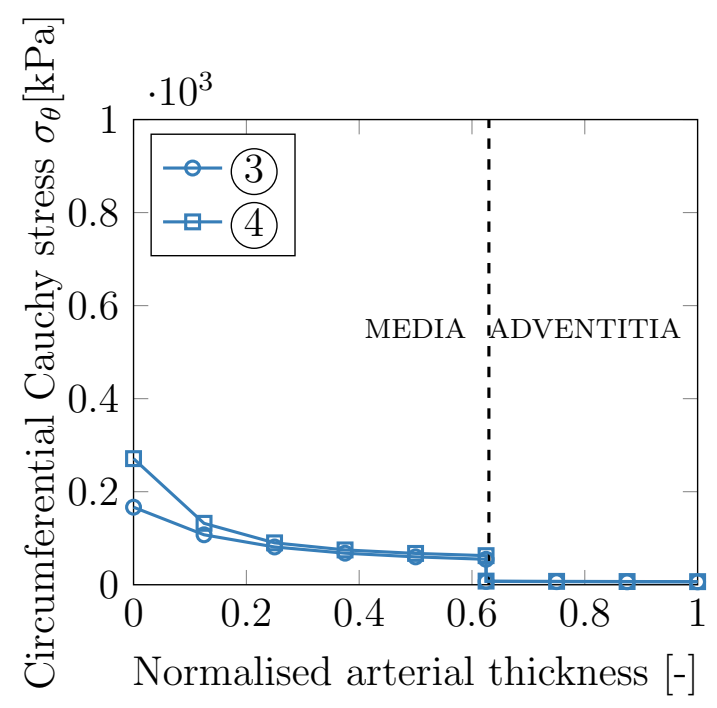

(d)

Figure 5.4: Balloon angioplasty. Load-displacement response of the artery in the intima at $Z=$ $0[\mathrm{~mm}]$ for Set 5 . The solid black curves represent the elastic response of the isotropic neo-Hookean matrix (lower curve) and the overall elastic response of the fibre-reinforced material (upper curve). The grey region represents the physiological blood pressure range, the white line represents the mean physiological blood pressure value. (a) Stressed-free tissue. (b) Residually stressed tissue. Distribution of circumferential stresses over the normalised thickness of the artery at $Z=0[\mathrm{~mm}]$ for Set 5. A sharp change in the stress distribution occurs at the interface between the media and the adventitia due to the different material properties on each layer. (c) Stressed-free tissue. (d) Residually stressed tissue.

measures the pressure exerted by the outer surface of the balloon on the inner surface of the artery. In the following, the grey region on the pressure-displacement diagrams represents the physiological blood pressure range under normal conditions (i.e. not hypertension), and the white line represents the mean value of the blood pressure, namely $13.33[\mathrm{kPa}]$. We 


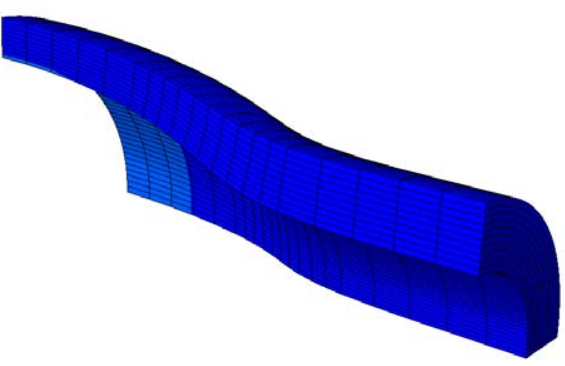

(a)

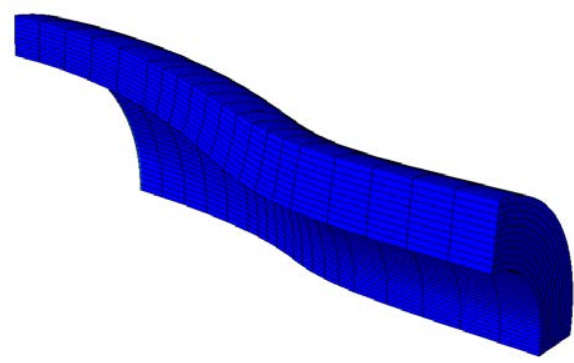

(c)

(d)

(b)

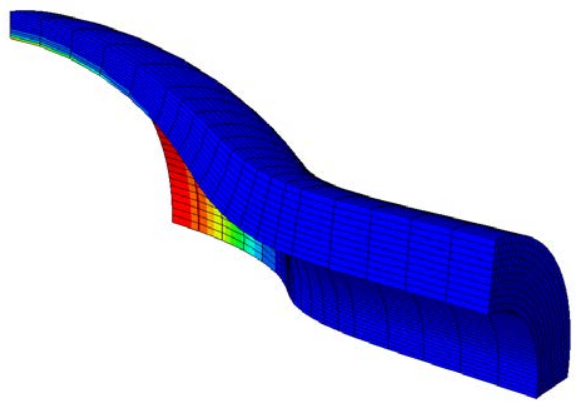

$\sigma_{\theta}[\mathrm{kPa}] 1 \times 10^{3}$
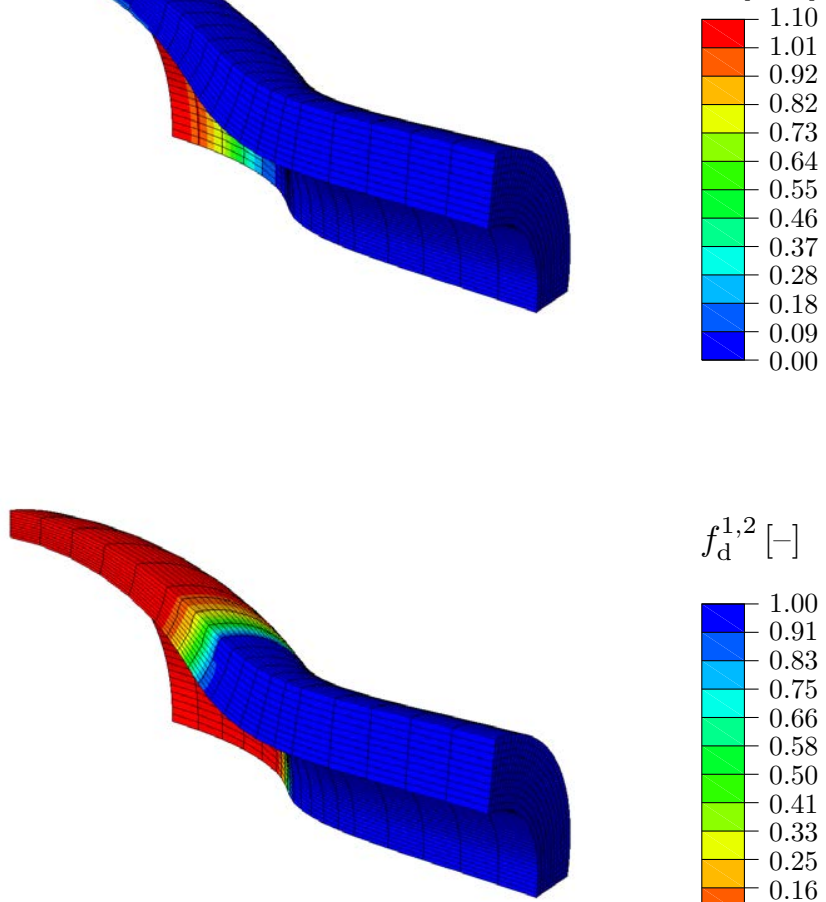

$f_{\mathrm{d}}^{1,2}[-]$

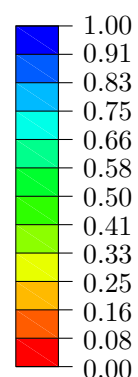

Figure 5.5: Balloon angioplasty. The geometry of the balloon is not included, and a mirror pattern is used to visualize one quarter of the full-3D geometry of the artery. Contour plots of the circumferential Cauchy stresses $\sigma_{\theta}$ and the damage function $f_{\mathrm{d}}$ for Set 5 in Figure 5.4(a). Residual stresses are taken into account. Figures (a) and (c) correspond to point (1) in the aforementioned figure. Figures (b) and (d) correspond to point (2) in the aforementioned figure.

first consider the purely elastic response without residual stresses. The dotted curves show the elastic response of the isotropic neo-Hookean matrix, while the solid curves show the overall elastic response of the fibre-reinforced material. The inclusion of the residual stresses causes the load-displacement curve to shift to the left. This is due to the contraction of the artery after the enforcement of the residual stresses. Although the solution presented in Section 5.1 should guarantee a $\boldsymbol{F}_{\text {res }}$ that satisfies the compatibility condition, this cannot occur in a domain with different material properties and different opening angle values, as is the case in this example of the balloon angioplasty. For this reason, the artery contracts until reaching a displacement at the inner surface of the intima of $u_{r \mathrm{i}}^{\mathrm{I}}=-0.157[\mathrm{~mm}]$, which finally explains why, in Figure 5.3c, the balloon in Case 3 makes contact with the artery earlier than in Case 2. In other words, the presence of the residual stresses results in smaller deformations of the arterial wall for the same load magnitude. This illustratively indicates the beneficial effect of residual stresses in arteries. This effect has recently been investigated in detail in Tobias Waffenschmidt [134] for a similar problem. 


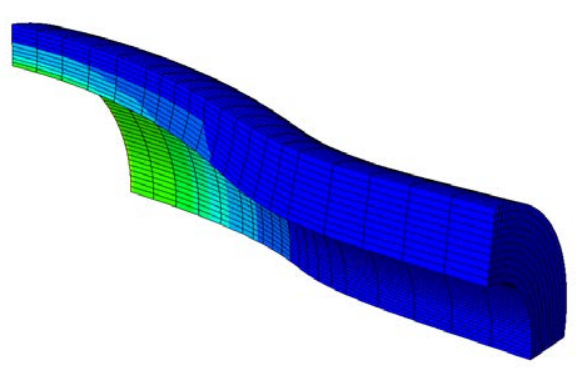

(a)

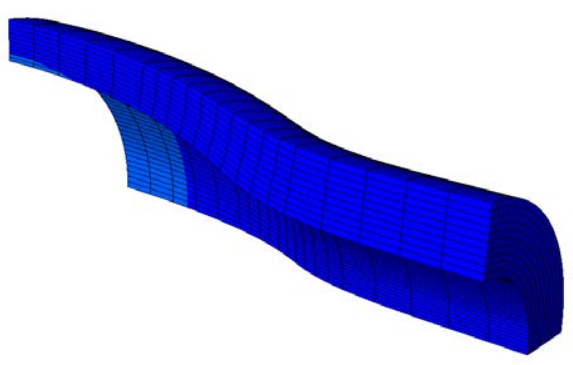

(c)

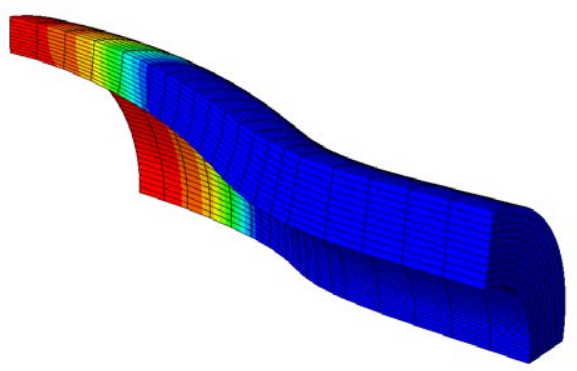

(d)

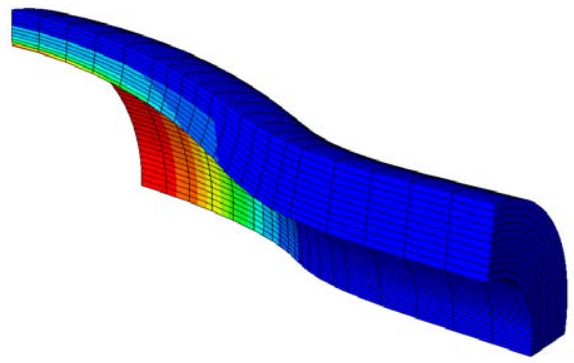

(b)

$\sigma_{\theta}[\mathrm{kPa}] 1 \times 10^{2}$
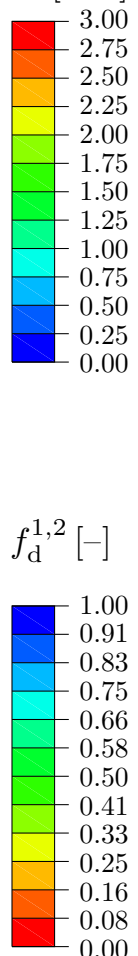

0.00

Figure 5.6: Balloon angioplasty. The geometry of the balloon is not included, and a mirror pattern is used to visualize one quarter of the full-3D geometry of the artery. Contour plots of the circumferential Cauchy stresses $\sigma_{\theta}$ and the damage function $f_{\mathrm{d}}$ for Set 5 in Figure 5.4(b). Residual stresses are taken into account. Figures (a) and (c) correspond to point (3) in the aforementioned figure. Figures (b) and (d) correspond to point (4) in the aforementioned figure.

Now that the characteristic elastic response of the balloon and the artery are explained, we focus on the inelastic response of the artery for different set of material parameters presented in Table 5.2. The material sets are chosen to highlight three distinctive structural responses in the artery. Sets 1-3, where damage evolves only in the matrix while the fibres remain elastic. Sets 4-6, where damage evolves only in the fibres while the matrix remains elastic. And sets 9-12, where damage evolves simultaneously in both, the matrix and the fibres. Before proceeding to study the whole spectrum of parameter sets, we focus on a single case to highlight, in the first place, the effect of the residual stresses in the response of the artery. Figures 5.4(a) and 5.4(b) show the load-displacement response of the artery for Set 5, without and with residual stresses, respectively. Two relevant remarks are to be made with respect to the afore-mentioned Figures. First, the load-displacement curves exactly follow the elastic response of the fibre-reinforced material (intact artery) up to the point where damage is triggered. Secondly, with regard to the effect of the residual stresses on the artery, we can see that the peak loads reached before the initiation of softening are 
higher when the residual stresses are taken into account. It is clear that the peak load lies below and above the maximum physiological pressure in Figures 5.4(a) and 5.4(b), respectively. Furthermore, points (1) and (2) mark the load state of the artery at the mean physiological pressure in Figure 5.4(a), before and after the initiation of softening, respectively. Meanwhile, points (3) and (4) mark the same load states in Figure 5.4(b). Figures 5.4(c) and 5.4(d) show the distribution of the circumferential stresses along the normalised thickness of the artery. In these figures we can appreciate that the effect of the residual stresses in the artery is twofold. On the one hand, the inclusion of the residual stresses reduces the gradient of circumferential stresses along the thickness of the artery. On the other hand, the inclusion of the residual stresses reduces the overall circumferential stress levels. From a mechanical point of view we can conclude from these observations that the residual stresses optimise the structural response of the artery. Finally, Figures 5.5 and 5.6 show the contour plots of the circumferential stresses and of the damage function in the artery at points (1) and (2), and (3) and (4), respectively. Again, it can be observed in these figures that the circumferential stresses in the residually stressed artery have a more homogeneous distribution and reach lower levels, than those attained in the non-residually stressed artery. Damage, similarly, is less pronounced in the residually stressed artery, while the non-residually stressed artery is more deformed and severely affected by damage. Now, we proceed with a comparative study of the different parameter combinations specified in Table 5.3.

In what follows, residual stresses are always taken into account. Figure 5.7(a) shows the load-displacement response of the artery for Sets 1-3. Two important facts are highlighted in this figure. First, the softening in the load-displacement curves is always followed by an exponential stiffening caused by the stretching of the elastic fibres. Secondly, the onset of damage is delayed when $\kappa_{\mathrm{d}}^{0}$ increases. This is an expected outcome, provided that equations 4.38 and 4.39 show that damage initiation in a particular phase $i$ has to fulfill the condition $q^{i}-\kappa_{\mathrm{d}}^{i}>0$, meaning that larger deformation states are required to reach the required $q^{i}$ to trigger the onset of damage. These two remarks serve to explain why the load-displacement curve of Set 3 almost exactly matches the curve of the elastic fibrereinforced material. For this particular case, the onset of damage is delayed up to the point where fibres are already sufficiently stretched, thus their contribution to the stiffness of the artery compensates for the damage in the matrix. Figure 5.7(b) shows similar results for Sets 4-6. The onset of damage is also delayed as the values of $\kappa_{\mathrm{d}}^{1,2}$ increase, nonetheless the stiffening that follows the softening phase is not as pronounced as for Sets 1-3. This is an expected outcome, since the fibres which are responsible for the exponential stiffening undergo damage. Furthermore, we are tempted to assume that, in the case of fibre-damage, the load-displacement curves should always drop onto the elastic neo-Hookean curve, as is the case in Waffenschmidt et al. [110], however the curves show a progressive stiffening. 


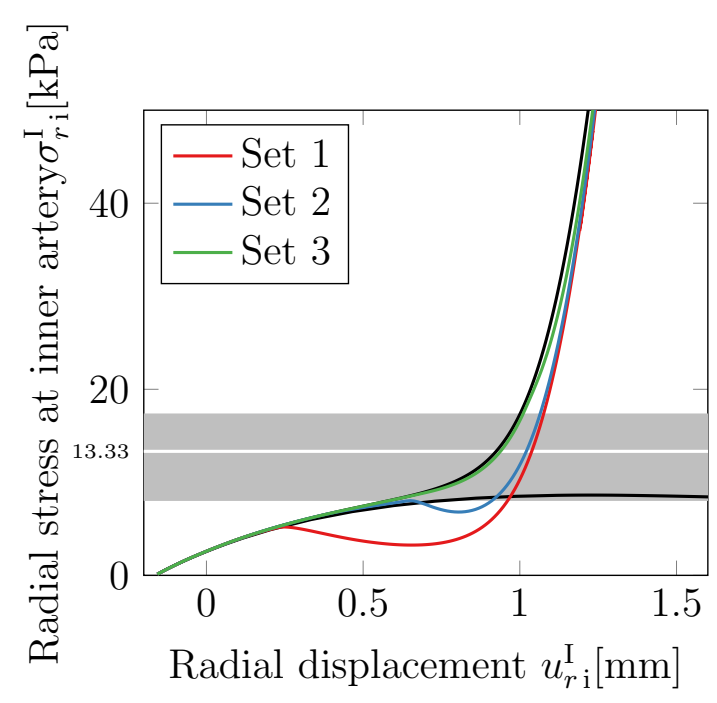

(a)

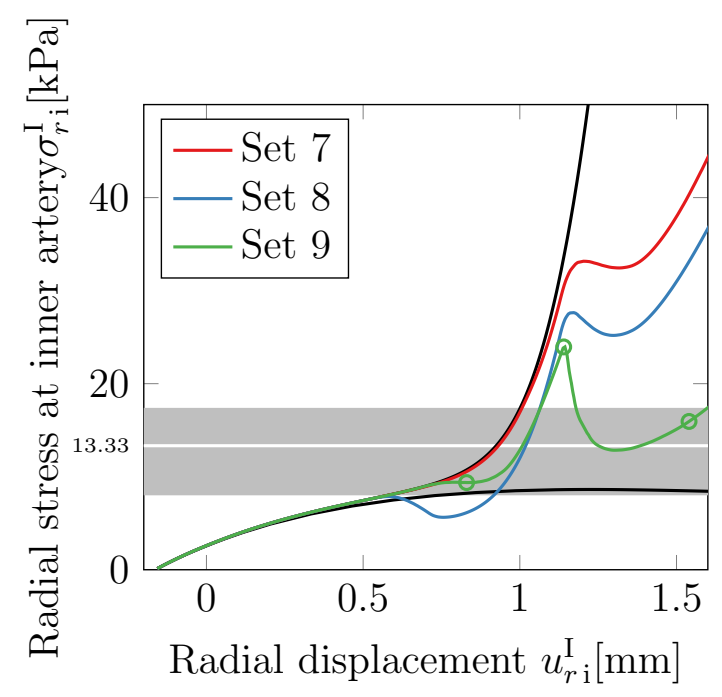

(c)

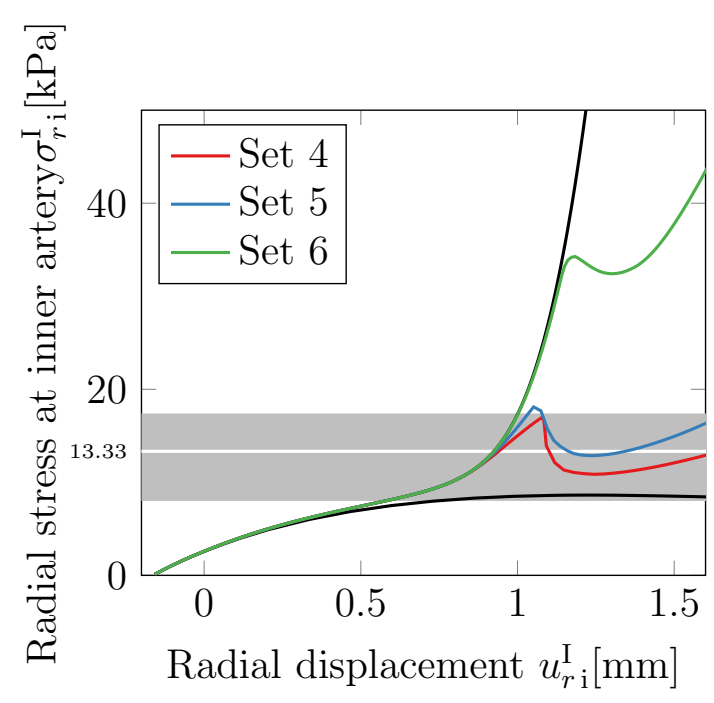

(b)

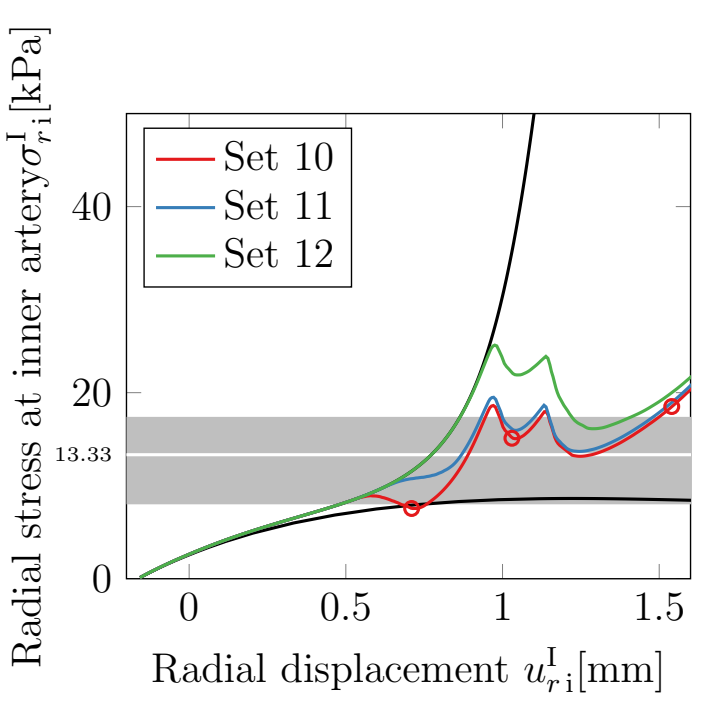

(d)

Figure 5.7: Balloon angioplasty. Load-displacement-response of the artery for: (a) Pure matrix damage. (b) Pure fibre damage. (c) Simultaneous damage in matrix and fibres. Symmetric fibre distribution. (d) Simultaneous damage in matrix and fibres. Unsymmetric fibre distribution. The solid black curves represent the elastic response of the isotropic neo-Hookean matrix (lower curve) and the overall elastic response of the fibre-reinforced material (upper curve). The grey region represents the physiological blood pressure range, the white line represents the mean physiological blood pressure value.

This situation can be better understood by observing figures 5.8 - 5.11, where the contour plots of the damage function are presented. There we notice that, close to the plane $Z=L[\mathrm{~mm}]$ the fibres are not damaged, which is the reason why they still contribute to the structural response of the artery and thus explaining the progressive stiffening in the load-displacement curves. Figure 5.7(c) shows the results for Sets 7-9. There we observe that for Sets 8 and 9 the load-displacement curve exhibits two regions with softening. To 


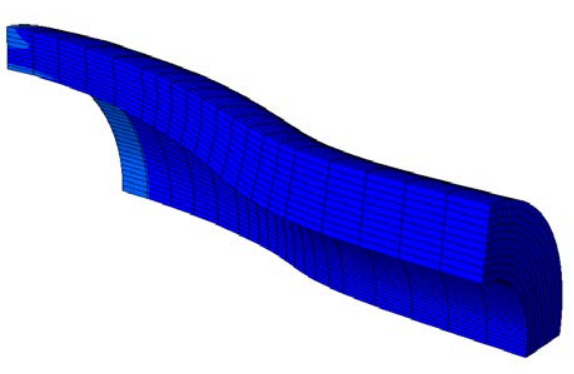

(a)

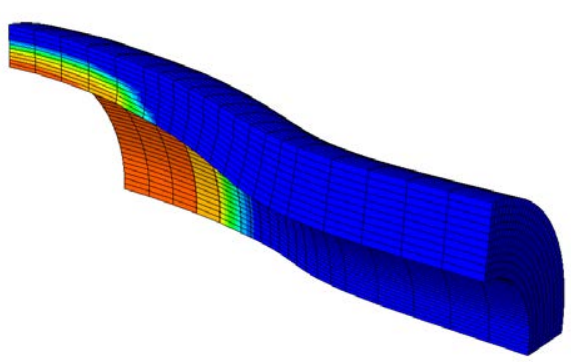

(d)

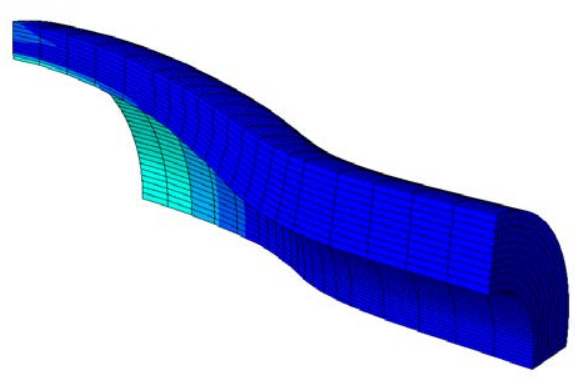

(b)

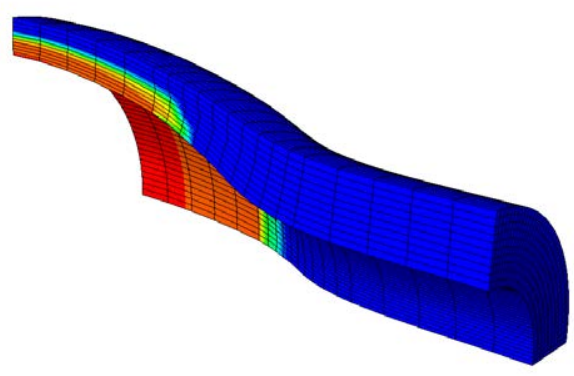

(e)

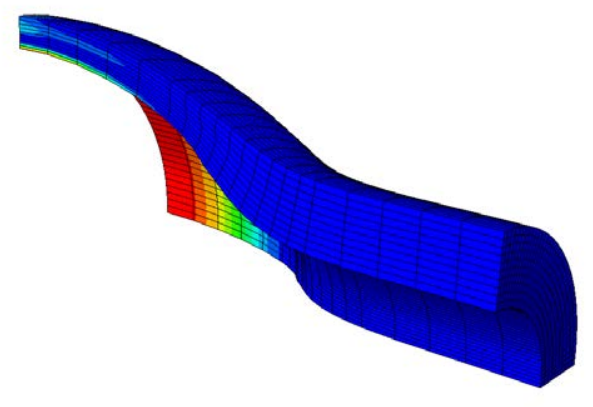

(c)

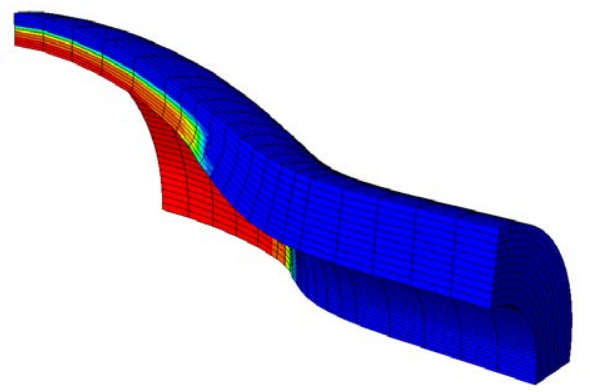

(f)
$\sigma_{\theta}[\mathrm{kPa}] 1 \times 10^{3}$
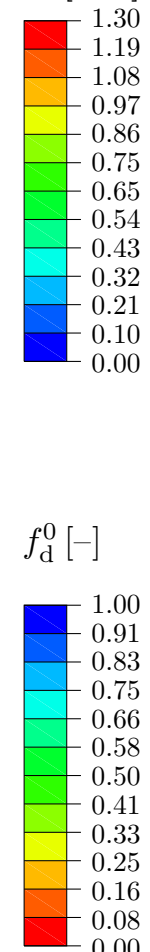

Figure 5.8: Balloon angioplasty for Set 9 in Figure 5.7(c). The geometry of the balloon is not included, and a mirror pattern is used to visualize one quarter of the full-3D geometry of the artery. Successive points in the load-displacement curve mark the instants at which the contour plots are displayed. The geometry of the balloon is not included, and a mirror pattern is used to visualize one quarter of the full-3D geometry of the artery. (a), (b) and (c) Contour plots of the circumferential Cauchy stresses $\sigma_{\theta}$. (d), (e) and (f) Contour plots of the damage function $f_{\mathrm{d}}^{0}$. 


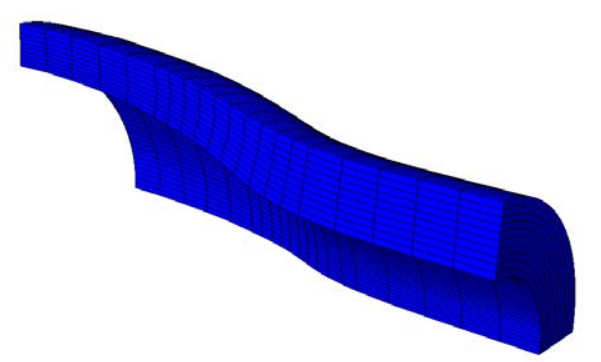

(a)

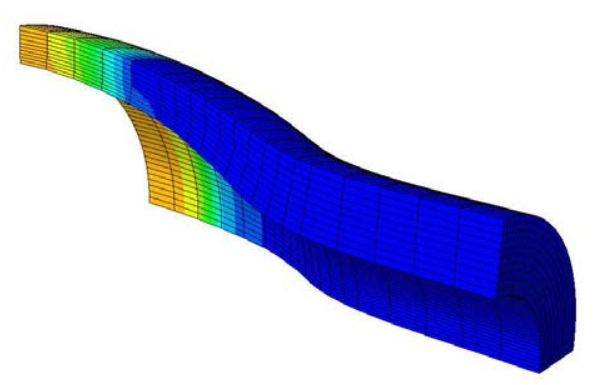

(b)

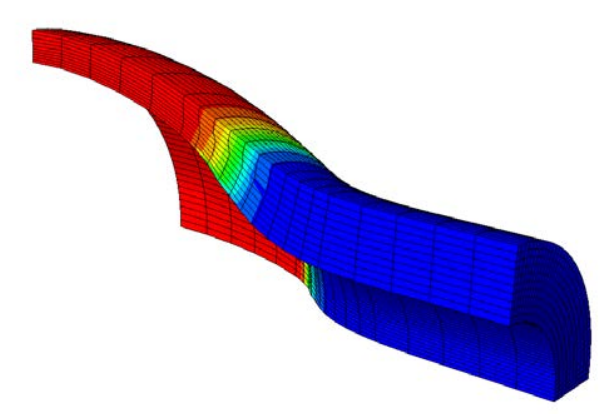

(c) $f_{\mathrm{d}}^{1,2}[-]$

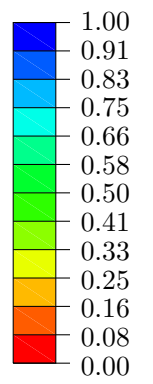

0.00

Figure 5.9: Balloon angioplasty for Set 9 in Figure 5.7(c). Successive points in the load-displacement curve mark the instants at which the contour plots are displayed. (a), (b) and (c) Contour plots of the damage function $f_{\mathrm{d}}^{1,2}$. 


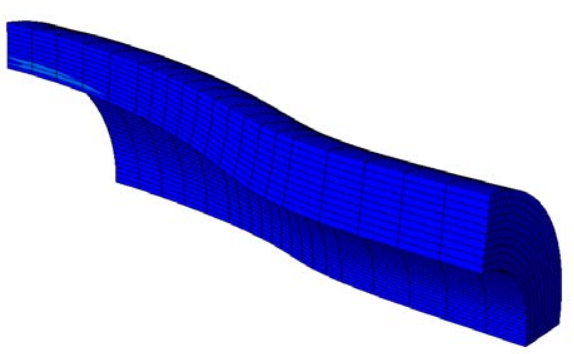

(a)

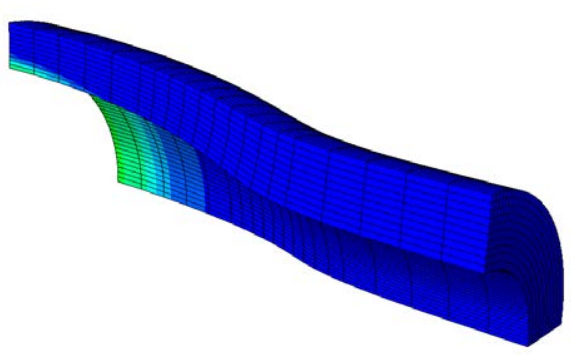

(d)

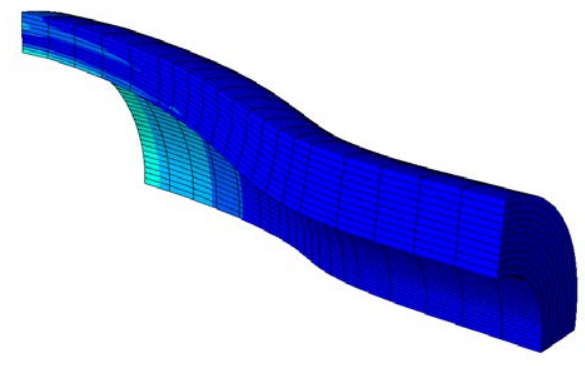

(b)

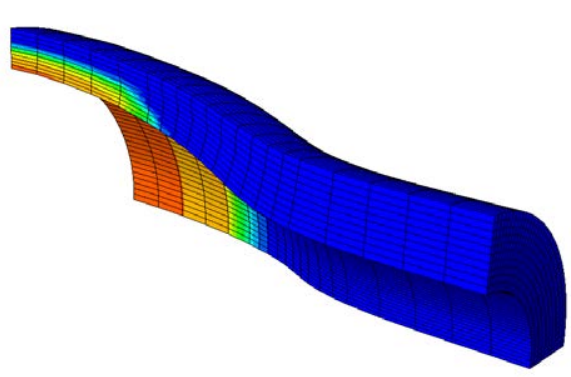

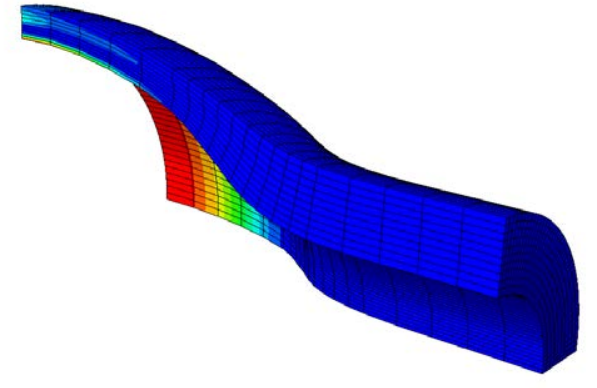

(c)

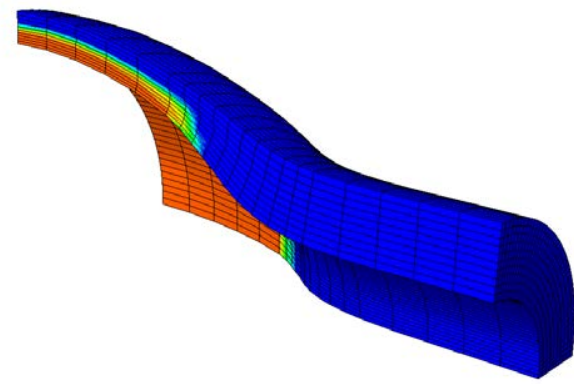

(f)
$\sigma_{\theta}[\mathrm{kPa}] 1 \times 10^{3}$

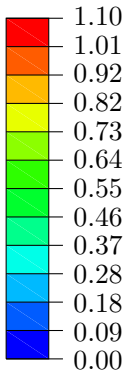

$f_{\mathrm{d}}^{0}[-]$

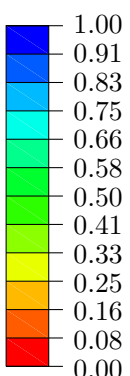

0.00

Figure 5.10: Balloon angioplasty for Set 10 in Figure 5.7(d). The geometry of the balloon is not included, and a mirror pattern is used to visualize one quarter of the full-3D geometry of the artery. Successive points in the load-displacement curve mark the instants at which the contour plots are displayed. The geometry of the balloon is not included, and a mirror pattern is used to visualize one quarter of the full-3D geometry of the artery. (a), (b) and (c) Contour plots of the circumferential Cauchy stresses $\sigma_{\theta}$. (d), (e) and (f) Contour plots of the damage function $f_{\mathrm{d}}^{0}$. 


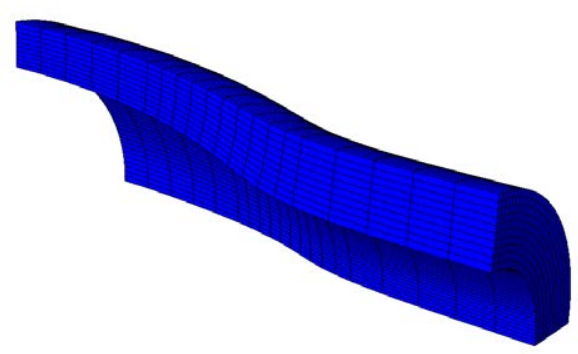

(a)

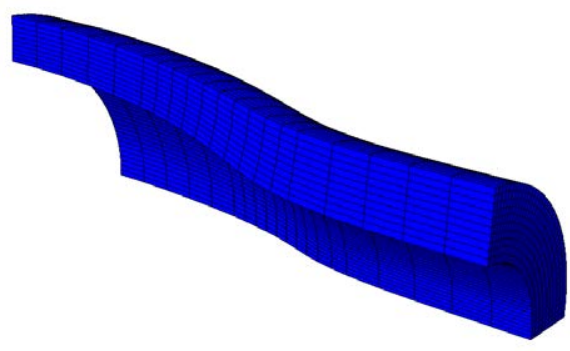

(d)

(e)
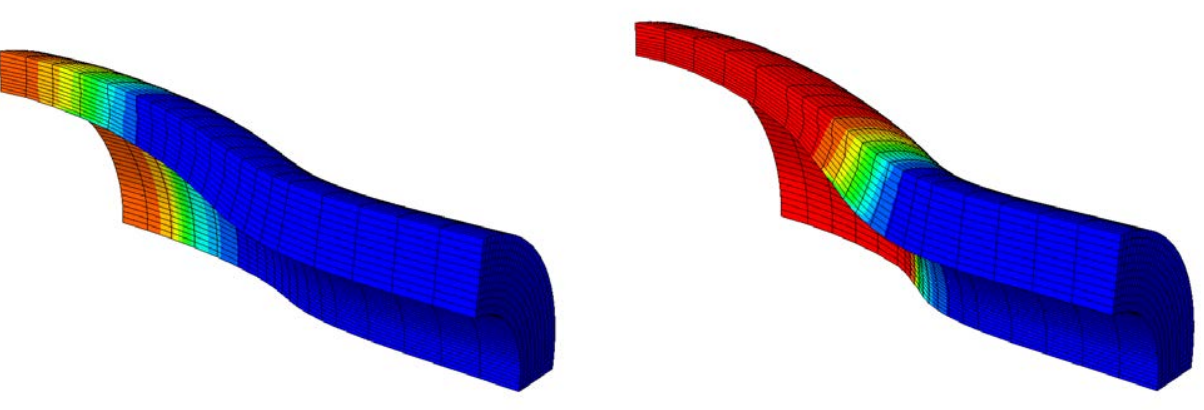

$f_{\mathrm{d}}^{2}[-]$

(f)

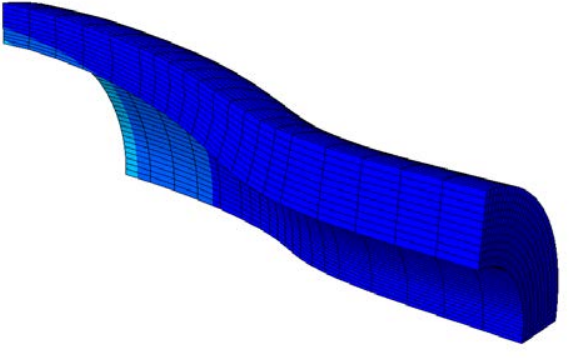

(b)

Figure 5.11: Balloon angioplasty for Set 10 in Figure 5.7(d). The geometry of the balloon is not included, and a mirror pattern is used to visualize one quarter of the full-3D geometry of the artery. Successive points in the load-displacement curve mark the instants at which the contour plots are displayed. The geometry of the balloon is not included, and a mirror pattern is used to visualize one quarter of the full-3D geometry of the artery. (a), (b) and (c) Contour plots of the circumferential Cauchy stresses $\sigma_{\theta}$. (d), (e) and (f) Contour plots of the damage function $f_{\mathrm{d}}^{0}$. 
understand this characteristic behaviour we must pay attention to the contour plots of figures 5.8 - 5.9, which correspond to the three different deformation stages marked in Figure $5.7 \mathrm{c}$ for Set 8 . From the sequence of contour plots, we observe that damage is first triggered in the matrix, and later on in both fibre families simultaneously due to their symmetric orientation. Going back to Figure 5.7(c), it can be stated that the first drop in the curves for both, Set 8 and 9, occurs as a consequence of damage initiation in the matrix, while the second drop is due to damage initiation in the fibres. As for Set 6 , we do not observe any significant softening in the curve due to matrix damage, on the account of the large value of the regularisation parameter $c_{\mathrm{d}}^{0}$ used.

So far we have limited ourselves to the the particular case of a symmetric fibre distribution within the artery, which is at the same time represented by a circular sector of the complete geometry, i.e. wedge. In order to be consistent with this simplification, i.e. the geometrical representation by means of a wedge and the symmetric fibre distribution, a set of boundary conditions is defined, which do not allow for unsymmetric deformations or deformations including a torsional mode, posing some restrictions on the variety of scenarios we can study. For this reason we now consider an unsymmetric fibre distribution to illustratively highlight that the fibre families do not degrade simultaneously but successively when undergoing different loading histories. For this purpose we modify the fibre angle of the second fibre family for the media from $\beta^{2, \mathrm{M}}=-21.7$ [deg] to $0.5 \beta^{2, \mathrm{M}}=-10.85$ [deg] and for the adventitia from $\beta^{2, \mathrm{~A}}=-62.26$ [deg] to $0.5 \beta^{2, \mathrm{~A}}=-31.13$ [deg].

The load-displacement response in the artery for such an unsymmetric fibre distribution is shown in Figure 5.7(d) for Sets 10-12. In contrast to the previous example, we observe a characteristic double peak which illustratively indicates that the fibre families do not degrade simultaneously but successively. We also observe a significant reduction in the peak stresses reached during the loading history. The associated damage evolution in the artery with unsymmetric fibre distribution is displayed for the three different deformation stages marked in Figure 5.7(d) for Set 10. In contrast to the previous case with symmetric fibre distribution, we obtain different damage evolution in both fibre families. While damage is already activated in the second fibre family, see Figure 5.11(e), the first fibre family remains undamaged at this deformation stage, cp. Figure 5.11(b). This illustratively shows that the first stress drop in Figure 5.7(d) is due to the second fibre family where damage is initiated first, followed by the first fibre family where damage is induced later. 


\subsection{Finite element modelling of a full-3d artery-like tube}

As a final example we present the problem of the inflation of a full-3d artery-like tube. The main purpose of this problem is to illustrate a more general scenario where each phase $i$ of the fibre-reinforced material experiences a different damage evolution. In order to achieve such a structural response, a perturbation in the fibre orientation is introduced, such that the fibre families at every material point undergo a different loading history. This example illustrates the potential of the proposed non-local gradient-enhanced damage model in Chapter 4 to be used in the study of real patient-specific arterial geometries, where the ground substrate and the two collagen fibre families in the arterial tissue generally undergo different loading histories.

\subsubsection{Setup of the boundary value problem}

We now present the problem of the inflation of a full-3d artery-like tube. The geometric and elastic material parameters of the tube correspond to those of the artery as defined in Table 5.2. The intima, though, is not accounted for. Instead, it is assumed that the tube is made only of the media and the adventitia, where the media has a thickness of $0.57[\mathrm{~mm}]$, taking over the region that was previously regarded as the intima. The tube is discretised by means of a mesh that has 11 elements along the thickness, 20 elements along the axial direction, and 40 elements in the circumferential direction, making a total of 17600 elements. However, in the contour plots presented later in the paper, only one half of the mesh is displayed in order to better visualise the contour plots along the thickness of the tube, even though the full-3D mesh is used for the simulation. The damage-related material parameters correspond to those of the artery as defined in Table 5.3. Two cases are studied, Sets 13 and 14, respectively. In the first case the matrix is regarded as elastic, while in the second one damage in both, the matrix and the fibres, is allowed to evolve. Furthermore, residual stresses are also taken into account. Regarding the boundary conditions, the tube is supported in the planes $Z=0[\mathrm{~mm}]$ and $Z=L[\mathrm{~mm}]$. There, the axial and circumferential displacements are fixed, while only radial displacements are allowed. The tube is then inflated by applying a pressure on its inner surface. Here, the arc-length method is used in order to capture the snap-back that occurs in the load-displacement response (See Waffenschmidt et al. [110]).

The main purpose of this problem is to illustrate a more general scenario where each phase $i$ of the fibre-reinforced material experiences a different damage evolution. In order to achieve such a structural response, a perturbation in the fibre orientation is introduced, such that the fibre families at every material point undergo a different loading history. The 
perturbation in the fibre orientation is defined by means of a perturbation angle $\beta_{\text {pert }}$ that varies along the $Z$-axis according to $\beta_{\text {pert }}=10+10 \sin (2 \pi \cdot Z / L)$. The fibre orientations in the media and adventitia are then modified to $\beta^{i} \rightarrow \beta^{i}+\beta_{\text {pert }}$. The variation of the fibre orientation along the $Z$-axis in the referential configuration is shown in Figure 5.12(a). As a measure of the degree of material anisotropy in the tube, we introduce the scalar quantity $P_{\boldsymbol{a}_{0}^{i}}=\boldsymbol{a}_{0}^{i} \cdot \hat{\boldsymbol{e}}_{\theta}$, which is the projection of the material unit vectors associated to each of the $i$ th-fibre families onto the circumferential unit vector. The contour plots of this quantity are presented in Figures 5.13(a) and 5.13(b) for the first and second fibre families, respectively. For a fully-transversely isotropic material this quantity should be equal for both fibre families, nonetheless in the Figures we appreciate differences due to the perturbation in the fibre orientation. Since the perturbation angle is only a function of the $Z$-coordinate, an axisymmetric distribution of $P_{\boldsymbol{a}_{0}^{i}}$ is obtained at each point along the $Z$-axis. Furthermore, we introduce the scalar quantity $P_{\boldsymbol{n}_{I I}}=\boldsymbol{n}_{I I} \cdot \hat{\boldsymbol{e}}_{Z}$, which is also a measure of the degree of material anisotropy. This quantity represents the projection of the unit vector associated to the second principal stress direction, $\boldsymbol{n}_{I I}$, onto the axial unit vector, $\hat{\boldsymbol{e}}_{Z}$. For a completely transversely isotropic elastic material -no damage taken into account- and under the specific boundary conditions given in this problem, $\boldsymbol{n}_{I I}$ and $\hat{\boldsymbol{e}}_{Z}$ are always parallel, meaning that $\boldsymbol{n}_{I I} \cdot \hat{\boldsymbol{e}}_{Z}=1$. Any deviation from this limiting value, thus, can be understood as a measure of the degree of anisotropy in the structural response. Figure 5.13(c) shows the contour plot of $P_{\boldsymbol{n}_{I I}}$ for the elastic tube subjected to a internal pressure of $p=20[\mathrm{kPa}]$. The plot corresponds to the undeformed geometry in order to better compare this new measure of material anisotropy with the previously defined $P_{\boldsymbol{a}_{0}^{1}}$ and $P_{\boldsymbol{a}_{0}^{2}}$. We can see that, within the media, the degree of anisotropy triggered by the fibre perturbation is negligible, whereas a strong variation of $P \boldsymbol{n}_{I I}$ along the $Z$-axis is obtained in the adventitia.

\subsubsection{Results}

The mechanical response of the full-3D artery-like tube is represented by the load-displacement diagram in Figure 5.12(b), where we plot the internal pressure $p$ against the radial displacement of a node lying at the inner surface of the tube and at $Z=0[\mathrm{~mm}]$, namely $u_{r, \mathrm{i}}$. Following the same convention from previous plots, the two limiting elastic cases, namely the load-displacement response of the neo-Hookean and the fibre-reinforced material, respectively, are represented by solid black curves. The same characteristic softening behaviour obtained for the artery in the balloon angioplasty can also be observed here for Sets 13 and 14. It is also worth mentioning that for both cases the solution was terminated due to convergence problems on the global system of equations. For Set 13, where the matrix remains elastic, a sharp drop in the load-displacement curve is appreciated close to 

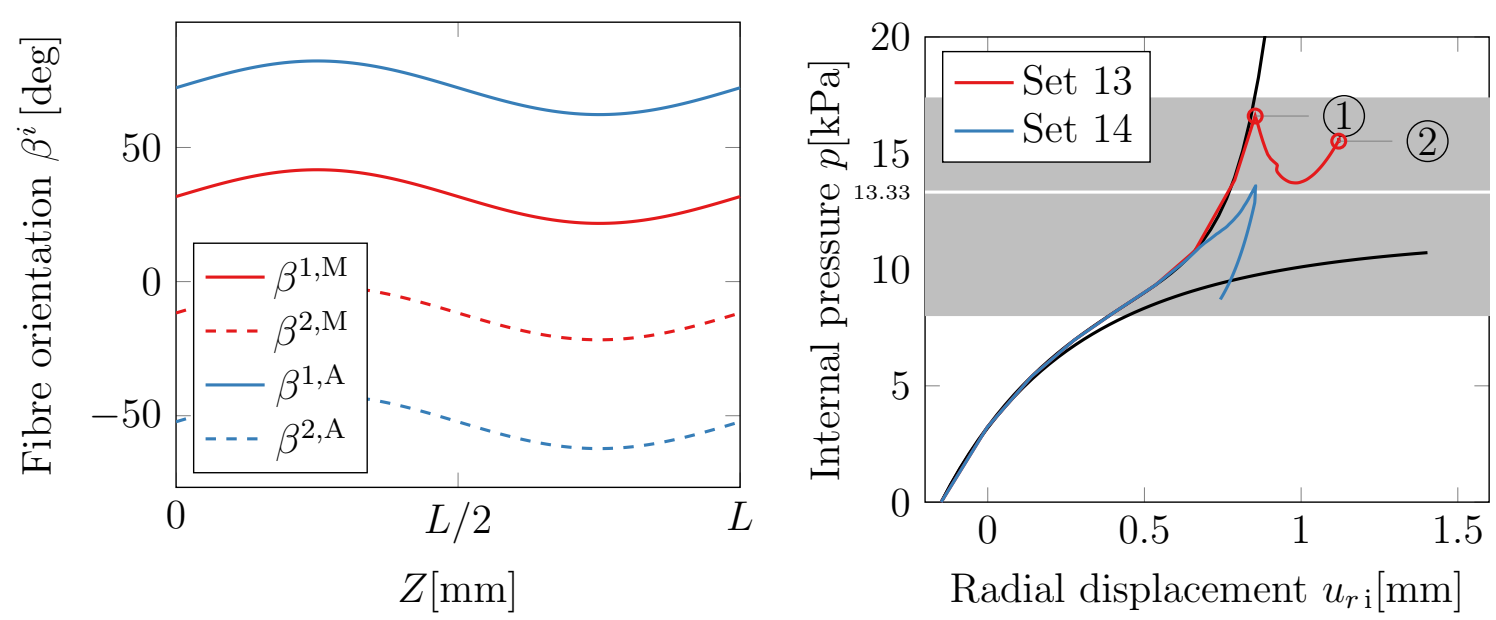

(a)

(b)
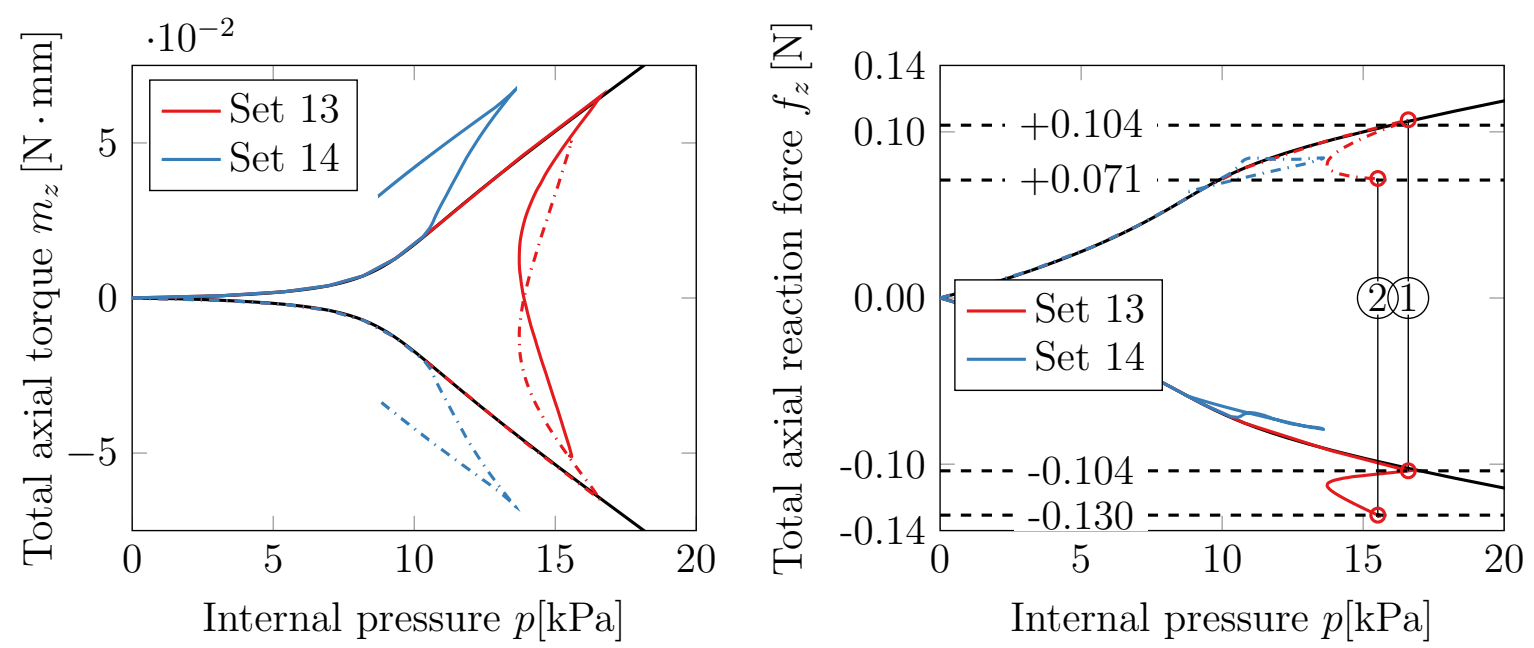

(c)

(d)

Figure 5.12: Full-3D artery-like tube under internal pressure. (a) Variation of fibre orientation along the $Z$-axis in the referential configuration. (b) Load-displacement-response. The solid black curves represent the elastic response of the isotropic neo-Hookean matrix (lower curve) and the overall elastic response of the fibre-reinforced material (upper curve). The grey region represents the physiological blood pressure range, the white line represents the mean physiological blood pressure value. (c) Total torque around $Z$-axis, $m_{Z}$. The solid black curves represent the elastic response of the fibre-reinforced material. (d) Total axial reaction force, $f_{Z}$. The solid black curves represent the elastic response of the fibre-reinforced material.

the upper limit of the physiological blood pressure, due mainly to the evolution of damage in the second fibre family. The softening in the response is followed later on by a continuous stiffening due mainly to the contribution of the first fibre family which is marginally affected by damage. This observation can be better appreciated in Figures 5.14(a)-(c). There, the contour plots show no damage evolution in the matrix, as expected, while damage is mostly concentrated in the second fibre family. For Set 14, damage evolves in both, the matrix and the fibres. In the load-displacement curve a first deviation from the elastic 


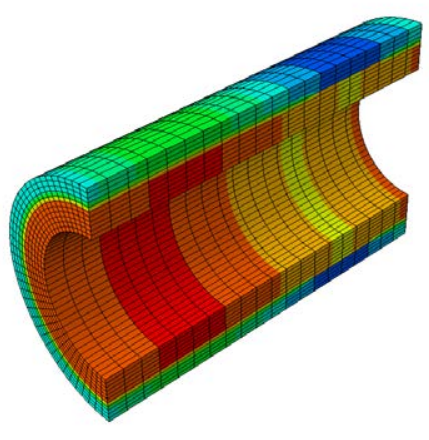

(a)

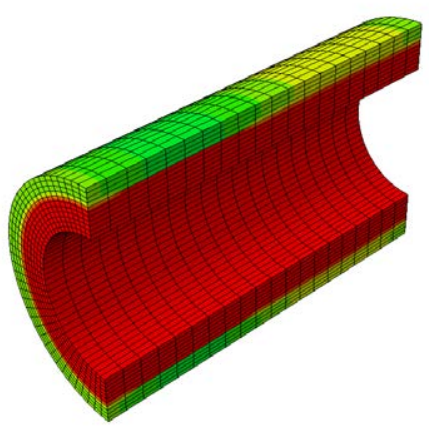

(b)

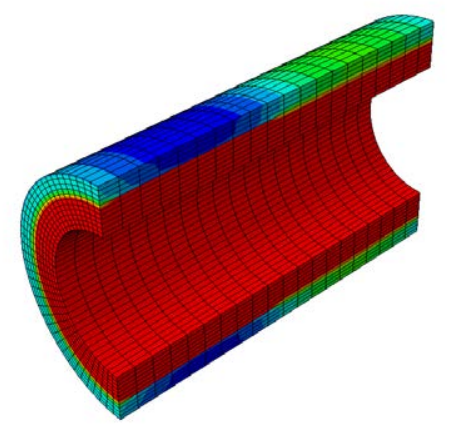

(c)

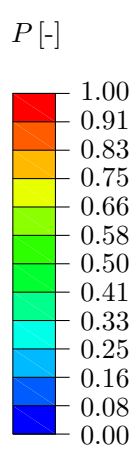

0.00

Figure 5.13: Full-3D artery-like tube under internal pressure. Quantities used to characterize the anisotropy of the material and the structural behaviour of the artery-like tube. Projection of the referential anisotropy direction vectors onto the circumferential unit vector. (a) $P_{\boldsymbol{a}_{0}^{1}}=\boldsymbol{a}_{0}^{1} \cdot \hat{\boldsymbol{e}_{\theta}}$. (b) $P_{\boldsymbol{a}_{0}^{2}}=\boldsymbol{a}_{0}^{2} \cdot \hat{\boldsymbol{e}_{\theta}}$. (c) Projection of the direction vector of the second principal Cauchy stress onto the axial unit vector, $P_{\boldsymbol{n}_{I I}}=\boldsymbol{n}_{I I} \cdot \hat{\boldsymbol{e}_{Z}}$. 
response occurs shortly after the pressure surpasses $10[\mathrm{kPa}]$ due to the onset of damage in the matrix. Later on, softening becomes dominant when the load surpasses the mean physiological blood pressure and damage considerably affects the second fibre family. This observation can be better appreciated in Figures 5.14(d)-(f). There, the contour plots show considerable damage in the matrix of the media, as well as in the second fibre family in the media and the adventitia, close to the plane $Z=0[\mathrm{~mm}]$. As it is the case for Set 13 , here the first fibre family is also marginally affected by damage. Furthermore, the perturbation in the fibre orientation induces torsional modes of deformation, and thus reaction torques appear at the supports. Figure 5.12(c) shows the reaction torques around the $Z$-axis, namely $m_{Z}$. It is observed that $m_{Z}$ is negligible for low pressure values since fibres are not sufficiently stretched, and that later on $m_{Z}$ evolves in each support symmetrically, i.e., with equal magnitude but different sign, as required to satisfy equilibrium. Figure 5.12(d), on the other hand, shows the reaction forces in the $Z$-axis, namely $f_{Z}$. From this Figure it becomes apparent that symmetry on $f_{Z}$ is broken after the onset of damage, which, in principle, suggests that equilibrium is not satisfied. In order to further study this apparent inconsistency in the results, we focus our attention on the load-displacement curve of Set 13, Figure 5.12(b). There we define points (1) and (2), which mark the onset of damage and the last converged solution in the simulation, respectively. Now, we turn our attention to Figure 5.15(a). There, the radial displacement $u_{r, \mathrm{i}}$ of nodes on the inner surface of the tube at (1) and (2) are presented. Provided the displacement field shows negligible variations along the circumferential direction, only representative values of the nodes with material coordinates $\left(r_{\mathrm{i}}, 0, Z\right)$ are considered. The resulting profiles show the variation of the radial displacement along the $Z$-axis. In (1), the profile of $u_{r, \mathrm{i}}$ is symmetric with respect to the plane $Z=L / 2[\mathrm{~mm}]$, hence the internal pressure does not produce a resultant force in the $Z$-axis. In (2), a different situation occurs. It is observed that within the last three quarters of the tube, i.e. for $Z \in[L / 4, L]$, the internal pressure exerts a net resultant force in the $Z$-axis. In order to calculate this resultant force, we first compute the area that results from the projection of the internal surface on the $Z$-plane. This area is computed by considering the internal radius of the tube at $Z=L / 4[\mathrm{~mm}]$ and $Z=L[\mathrm{~mm}]$, namely $r_{\mathrm{i}, L / 4}=R_{\mathrm{i}}+\left.u_{\mathrm{i}}\right|_{Z=L / 4}$ and $r_{\mathrm{i}, L}=R_{\mathrm{i}}+\left.u_{\mathrm{i}}\right|_{Z=L}$, respectively. With these values the area is then calculated, $A=\pi \cdot\left[r_{\mathrm{i}, L / 4}^{2}-r_{\mathrm{i}, L}^{2}\right]=3.79\left[\mathrm{~mm}^{2}\right]$. Finally, the resultant force acting on the inner surface of the tube is computed. The pressure at (2) is $p=15.52[\mathrm{kPa}]$, which produces a net positive resultant force $f_{Z}=p \cdot A=+0.06[\mathrm{~N}]$. Taking this value into account, one can clearly see that the reaction forces acting on the structure, namely $f_{Z}=-0.13[\mathrm{~N}]$ at $Z=0[\mathrm{~mm}]$, and $f_{Z}=+0.07[\mathrm{~N}]$ at $Z=L[\mathrm{~mm}]$, are properly balanced. A final observation is made regarding the distribution of stresses along the thickness of the tube. The asymmetric distribution in the reaction forces $f_{Z}$, naturally favors a stress concentration on $Z=0[\mathrm{~mm}]$. In Figure $5.15(\mathrm{~b})$ we see the distribution of the axial com- 
ponent of the Cauchy stress tensor, $\sigma_{z}$, along the normalized thickness of the tube at its supports. In this Figure we first note a jump in the stress distribution at the interface between the media and the adventitia due to the different elastic material properties in each layer. Within the adventitia, we observe that the stress distribution remains almost unchanged between (1) and (2), and furthermore, that stress levels in both supports are practically identical. Within the media, on the other hand, while the stress levels at (1) have similar values on both supports, at (2) a major deviation in the stress distribution occurs. At this point, $\sigma_{z}$ significantly increases at $Z=0[\mathrm{~mm}]$, reaching a maximum value of $22[\mathrm{kPa}]$ at the inner surface of the tube, while at the same time, $\sigma_{z}$ decreases consistently at $Z=L[\mathrm{~mm}]$, reaching a maximum value of $10[\mathrm{kPa}]$ at the interface between the media and the adventitia.

\subsection{Summary}

The balloon angioplasty is presented as an example of a biomechanical related application, and the evolution of damage in the artery is studied by means of the non-local gradientenhanced damage model presented in chapter 4. We assume an idealized configuration of two concentric hollow cylinders representing the balloon and the artery respectively. This idealized geometrical configuration is further simplified to a cylindrical sector, a wedge, which is used for the finite element representation of the problem (see Figure 5.2). This simplification enables us to use the procedure discussed in Section 5.1 to include the residual stresses within the artery. In a first stage the elastic response of the artery is studied. We pay special attention to the effects of the residual stresses on the artery and their implications for the contact problem. We observe in Figure 5.3(d) that the presence of the residual stresses result in smaller deformations of the arterial wall for the same load magnitude. The effect of the residual stresses on the inelastic response of the artery is also studied for Set 5. The results in figures 5.4 - 5.6 show that the inclusion of the residual stresses has three major consequences on the structural response of the artery. First, it allows to reach higher values of the peak pressure before entering into the softening regime. Secondly, it reduces the gradient of circumferential stresses along the thickness of the artery. Thirdly, it reduces the overall circumferential stress levels. We can conclude from these observations that the residual stresses optimise the structural response of the artery, which illustratively indicates the beneficial effect they have on the blood vessels. In a second stage, the evolution of damage is studied for the set parameter combinations in Table 5.2. Different scenarios are taken into consideration: purely matrix damage, purely fibre damage, and combined damage evolution in the matrix and the fibres. In general, it is possible to reproduce a loss of stiffness in the artery when the radial stresses exceed the physiological range. From the results it becomes apparent that when the artery is 


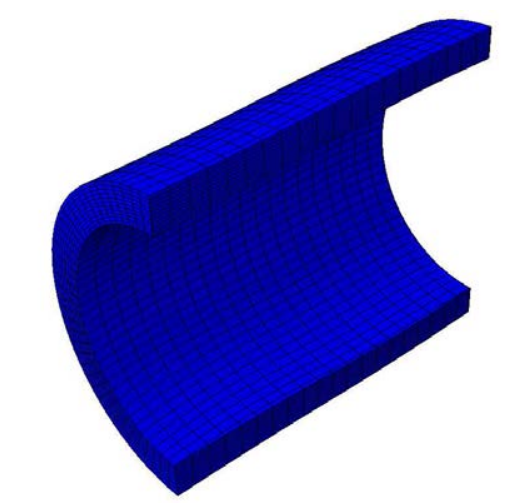

(a)

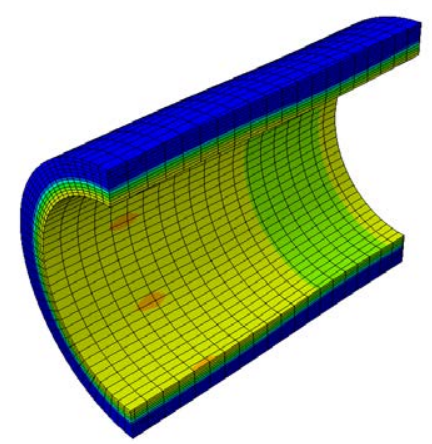

(d)

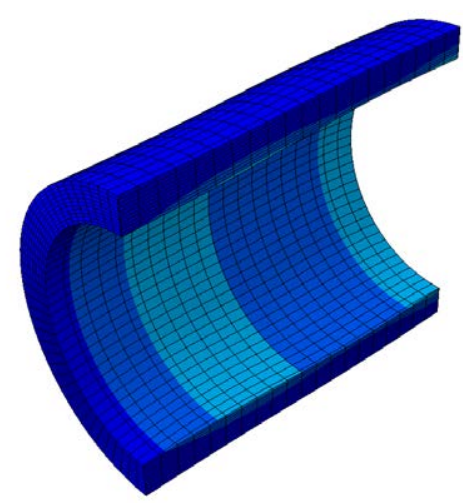

(b)

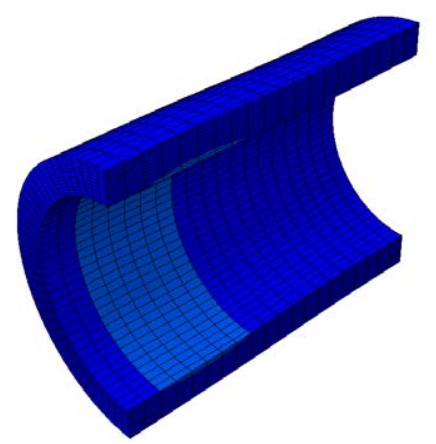

(e)

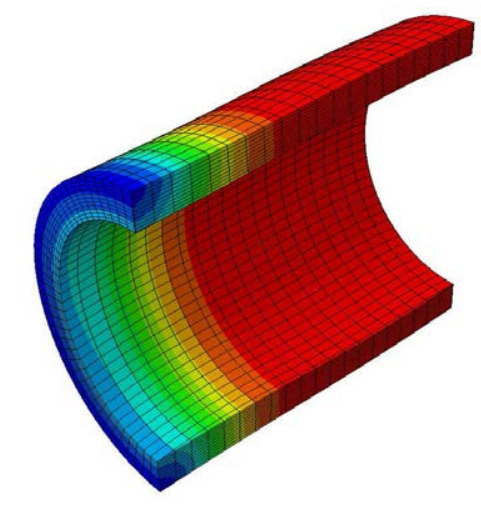

(c)

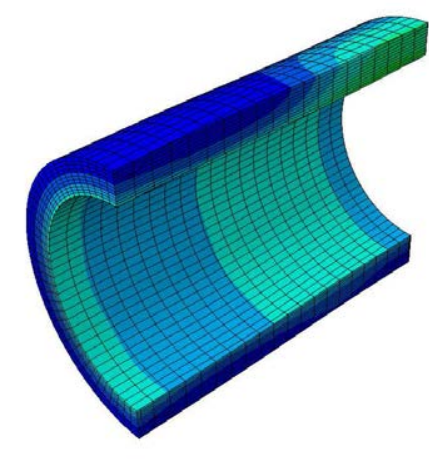

(f)

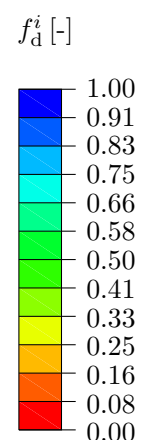

$f_{\mathrm{d}}^{i}[-]$

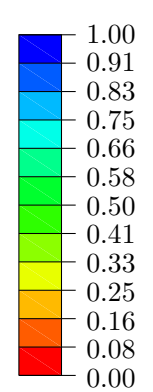

Figure 5.14: Full-3D artery-like tube under internal pressure. Contour plots of the damage function $f_{\mathrm{d}}^{i}$. Figures (a), (b) and (c) show the damage functions $f_{\mathrm{d}}^{0}, f_{\mathrm{d}}^{1}$ and $f_{\mathrm{d}}^{2}$, respectively, for Set 13 at the last load increment. Figures (d), (e) and (f) show the damage functions $f_{\mathrm{d}}^{0}, f_{\mathrm{d}}^{1}$ and $f_{\mathrm{d}}^{2}$, respectively, for Set 14 at the last load increment. 


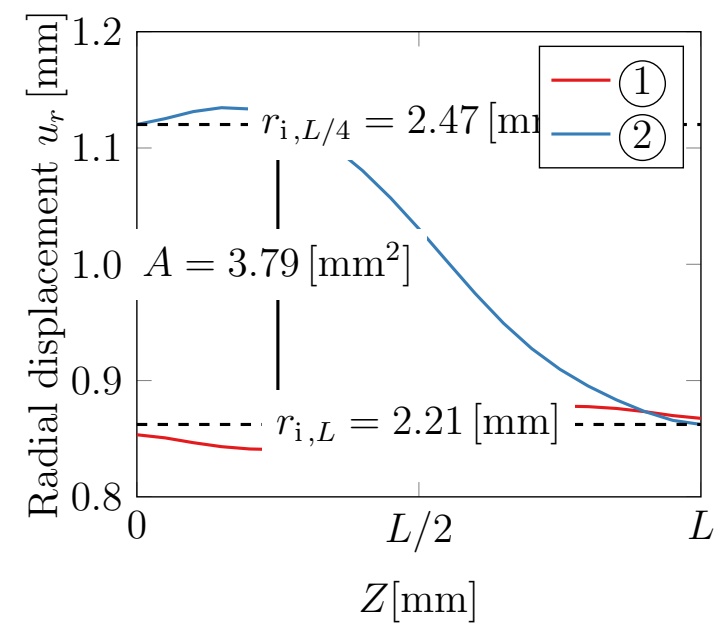

(a)

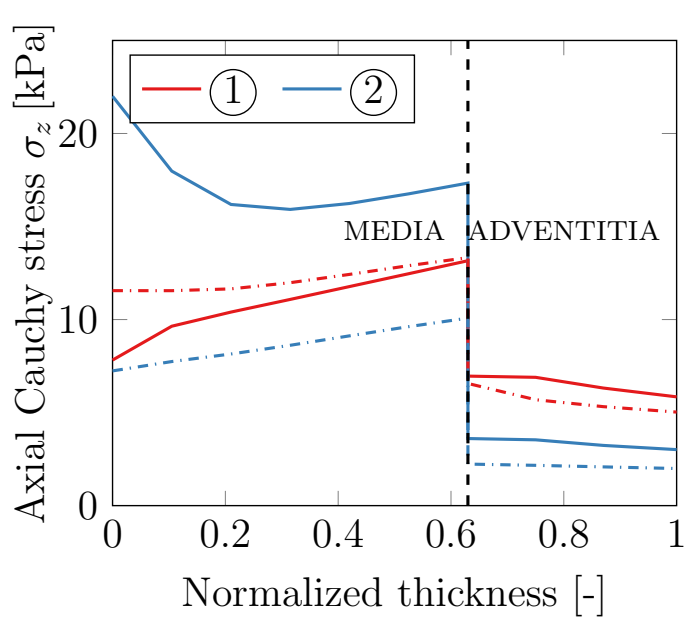

(b)

Figure 5.15: Full-3D artery-like tube under internal pressure. Plots of structural response at points (1) and (2) of the load-displacement curve for Set 13. (a) Profile of radial displacement of inner nodes along the $Z$-axis. (b) Distribution of axial Cauchy stress along the normalized thickness at the supports.

subjected to radial stresses beyond the physiological range, damage evolution is triggered in both, the matrix and the fibres, which causes an increase of the tube's diameter at the same mean pressure. Even though the artery loses stiffness, these damage effects can be beneficially used in medical surgery to expand an artery's diameter and thereby restore and ensure the blood flow of an atherosclerotic or arteriosclerotic blood vessel, for example. In contrast to the results presented in Waffenschmidt et al. [110] and Tobias Waffenschmidt [134], where similar problems were studied, for this case of the balloon angioplasty the structural response of the artery never reaches the purely neo-Hookean limit defined in the load-displacement diagrams, due to the fact that not all fibres are completely damaged and thus still contribute to the overall structural response. We conclude this application example considering an unsymmetric fibre distribution to illustratively highlight that the fibre families do not degrade simultaneously but successively when undergoing different loading histories.

The problem of the inflation of a full-3d artery-like tube is presented as a final application example. Contrary to the case of the balloon angioplasty, where the simplification of the geometry as a wedge approximation imposes the use of specific boundary conditions that do not allow to capture torsional modes of deformation, this problem illustrates a more general scenario where no particular restrictions on the load and boundary conditions are imposed. A perturbation in the fibre orientation is introduced, such that the fibre families at every material point undergo a different loading history, and hence each phase $i$ of the fibre-reinforced material experiences a different damage evolution. The evolution of damage is studied for the a two sets of different parameter combinations. In Figure 5.12(b) it is 
observed that for both cases we capture the softening effect within the physiological pressure range. In Figure 5.12(c) we observe the appearance of reaction torques $m_{Z}$ at the supports due to the development of torsional modes of deformation. Finally, in Figure 5.12(d), we observe the evolution of the reaction force $f_{Z}$ in the supports. Due to the unsymmetric evolution in damage, which is more concentrated towards plane $Z=0[\mathrm{~mm}]$, the tube is deformed in such a way that the pressure exerts a resultant force along the $Z$-axis, causing an unsymmetric evolution in the reaction forces $f_{Z}$ after the onset of damage. 


\subsection{Synthesis and conclusions}

The present thesis deals with the study of the mechanical behaviour of the aortic artery using non-linear finite element models. Here a state of the art hyperelastic constitutive formulation is used to characterise the distinctive biomechanical features of the arterial wall as presented by Fung [12] and Humphrey [13]. The hyperelastic constitutive formulation follows the works by Holzapfel et al. [72] and Gasser et al. [7], and accounts for the characteristic composite-like structure of blood vessels where two collagen fibre families are embedded in an isotropic extracellular matrix. By using structural tensors associated to two material unit vectors that represent the mean fibre orientations, we are able to capture the transversely isotropic behaviour that results from a helical distribution of collagen fibres in blood vessels, as well as the effect of collagen fibre dispersion. Furthermore, a multiplicative split of the deformation gradient tensor is used to characterise independently the volumetric and the isochoric deformation, hence allowing for the simulation of quasi-incompressible behaviour. Building on the aforementioned hyperelastic formulation, in this thesis we develop a framework to study the damage-related softening in blood vessels when the arterial wall is subjected to loads beyond its physiological range. Damage is then studied by means of two different approaches from which the main conclusions are presented in the following.

\subsubsection{Continuum local damage formulation with regularisation}

In chapter 3 we present a continuum local damage formulation with regularisation. The formulation aims to establish a relationship between the mesoscopic structural mechanisms that rule inelastic processes in fibrous soft tissues and their macroscopic material parameters. The principal results and conclusions are summarised in the following. 
- A phenomenological damage model for fibrous materials with regularised softening is developed. Meso-structurally based definitions for the macroscopic elastic parameters defining the effective strain energy function and for the inelastic parameters defining the ingredients of the damage model are obtained by means of a minimisation procedure using the SciPy library Jones et al. [107].

- Soft tissues are regarded as fibre reinforced composites and fibres as fibril reinforced composites. At the mesoscopic level a Hodge-Petruska bidimensional model (Petruska and Hodge [84]) is used to describe the fibrils as staggered arrays of tropocollagen molecules, and from this mesoscopic model the macroscopic material properties of the fibres using an homogenisation process are obtained.

- A smeared crack approach is used to avoid the mesh size dependence of the structural response in softening. A regularised softening modulus is defined in terms of a surface density of dissipated energy and a characteristic size of the finite element.

- The damage model is implemented by means of a material subroutine inside the finite element code FEAP. Taylor [106].

\subsubsection{Non-local gradient-enhanced continuum damage formula- tion}

In chapter 4 we present a non-local gradient-enhanced continuum damage formulation. The principal results and conclusions are summarised in the following.

- A non-local gradient-enhanced continuum damage formulation at large deformations is developed. The free energy function is enhanced with a term that contains the referential gradient of the non-local damage variable $\phi^{i}$, where $i=\{0,1,2\}$ refers to the phases that make up the arterial wall, namely the ground matrix and the two fibre families.

- The twofold effect of the regularisation parameter $c_{\mathrm{d}}^{i}$ is studied using the example of the plate with a whole. First, we study the variation on the dissipation, manifested on the overall structural response represented by the load-displacement curves. Secondly, we study the changes in the distribution of the non-local damage variable field $\phi^{i}$, which is more localised for low values of $c_{\mathrm{d}}^{i}$, while is more smeared over the domain for large values of $c_{\mathrm{d}}^{i}$.

- A framework that allows for the inclusion of residual stresses by means of a multiplicative composition of the deformation gradient is presented. This approach is based in the analytical solution of the opening-angle experiment. 
- The non-local damage model is implemented in the commercial finite-element software Abaqus/Standard by means of the user subroutine UEL. To this end a Q1P0 element is implemented following a standard multivariational approach in order to enforce the quasi-incompressibility of the soft tissues.

Furthermore, the capabilities of the non-local gradient-enhanced continuum damage formulation to the application of biomechanically related problems is explored by means of an illustrative example of a balloon angioplasty procedure. The results show that the proposed non-local damage formulation is able to capture the characteristic damage softening behaviour in blood vessels when the interior pressure exerted on the arterial wall surpasses tolerable physiological values. Finally we point out that the developed framework to include the residual stresses in blood vessels allows to illustrate the beneficial effect of the residual stresses in the overall structural behaviour of the arterial wall.

\subsection{Main contributions}

The main contributions of this thesis can be summarised as follows.

- A continuum local damage formulation with regularisation for soft tissues is introduced. The elastic and damage related material parameters are based on considerations of the mesostructure of soft tissues. The proposed model serves to capture damage-related softening related to collagen damage.

- A non-local gradient-enhanced continuum damage formulation for soft tissues is presented. The constitutive formulation that governs the elastic response is purely phenomenological and is able to capture the characteristic behaviour of human blood vessels. The damage formulation is able to capture the softening behaviour associated to collagen failure. Furthermore, the implicit gradient-enhanced regularisation guarantees mesh-objective results of the finite element implementation.

- The applicability of the non-local damage formulation to cardiovascular-related problems is explored by means of the simulation of an idealised balloon angioplasty configuration. The model is able to capture damage-softening when the arterial tissue is subjected to supraphysiological loads. The beneficial effects of residual stresses in the overall structural response of the blood vessel is also highlighted in this study.

\section{3 limitations and future perspectives}

In the development of the present thesis we have made several assumptions that allowed to solve complex problems with simple approximations. This considerations might limit the 
future applicability of the proposed framework to real clinical applications. With regard to the non-local damage formulation, there are still open issues worth exploring.

- Damage parameters that control the onset of damage, the saturation of the damage function, and the regularisation, have been chosen on a trial and error basis. Although the results obtained, for example, in the simulation of the balloon angioplasty, are consistent with the limits imposed by the physiological values of the blood pressure, i.e. we have seen that damage is triggered after surpassing the maximum value of the blood pressure, as expected, it is still necessary to calibrate the damage-related parameters using experimental data.

- The proposed framework allows for the inclusion of any particular format for the damage function, as long as it fulfills the required conditions of equation (4.2). So far we have used the same exponential format to reproduce the damage in the matrix as well as in the collagen fibres. Since these arterial constituents, from a mechanical point of view, are of a completely different nature, further investigation related to the use of different damage functions for the ground substrate is required.

- We have used spatial gradient of the non-local damage variable for the enhancement of the free energy. Another alternative, that could lead to a more robust finite element implementation would be to use the material gradient. The gradient term in equation (4.3) is formulated in the spatial configuration. The formulation of this gradient term in the material configuration should be further explored, since gradient based regularisation could be improved.

With regard to the local damage model, we should note that only very simple boundary value problems were solved. Further investigation about the performance of this formulation in more general scenarios is required.

Finally, the residual stresses are implemented by means of a multiplicative composition of the deformation gradient, which is in turn based on the analytical solution of the openingangle experiment. The analytical solution of the opening-angle experiment is limited to perfectly cylindrical geometries, meaning that this is not the best approach when dealing with real patient-specific geometries. New approaches should be considered to overcome this limitation.

\subsection{Publications}

The major results and models of this thesis have been published as papers in two peerreviewed journals, and one book chapter. 
- Sergio Blanco, César Polindara, Jose María Goicolea. A regularised continuum damage model based on the mesoscopic scale for soft tissue. International Journal of Solids and Structures, 2014.

- Tobias Waffenschmidt, César Polindara, Andreas Menzel, Sergio Blanco. A gradientenhanced large-deformation continuum damage model for fibre-reinforced materials. Computer Methods in Applied Mechanics and Engineering, 2014.

- Tobias Waffenschmidt, César Polindara, Andreas Menzel. A Gradient-Enhanced Continuum Damage Model for Residually Stressed Fibre-Reinforced Materials at Finite Strains. Biomedical Technology, Springer, 2014.

The author of this thesis has contributed for all the publications with the finite element implementation and the development of the numerical examples. In the second paper and the book chapter he also has contributed to the preparation of the manuscript. 


\section{Local tangent moduli}

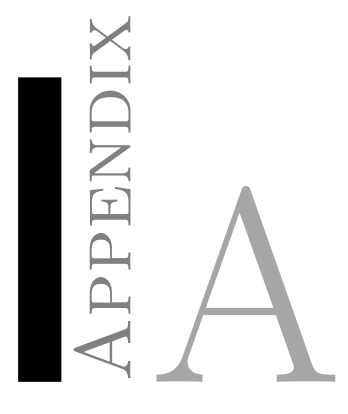

The local tangent moduli, contained in the element Jacobians can conveniently be derived in material description and then transferred to the spatial description as used for the numerical implementation, by means of push-forward operations. The expression herein presented are derived in a general way to apply to each of the phases $i=\{0,1,2\}$ that form the continua, namely the ground matrix and the two fibre families. In the following and for the sake of readability, we omit the subscript index $n+1$ associated with time $t_{n+1}$ as well as the superindex $i$ that denotes a specific phase.

We start by introducing the following expressions

$$
\begin{aligned}
\mathbf{E}:=2 \frac{\mathrm{d} \boldsymbol{S}}{\mathrm{d} \boldsymbol{C}} & =f_{\mathrm{d}} \overline{\mathbf{E}}+2 \overline{\boldsymbol{S}} \otimes \frac{\partial f_{\mathrm{d}}}{\partial \kappa} \frac{\partial \kappa}{\partial \boldsymbol{C}}, \\
\frac{\mathrm{d} \boldsymbol{S}}{\mathrm{d} \phi} & =\frac{\partial f_{\mathrm{d}}}{\partial \kappa} \frac{\partial \kappa}{\partial \phi} \overline{\boldsymbol{S}} \\
2 \frac{\mathrm{d} Y}{\mathrm{~d} \boldsymbol{C}} & =2 \frac{\partial Y}{\partial \kappa} \frac{\partial \kappa}{\partial \boldsymbol{C}}, \\
\frac{\mathrm{d} Y}{\mathrm{~d} \phi} & =\frac{\partial Y}{\partial \phi}+\frac{\partial Y}{\partial \kappa} \frac{\partial \kappa}{\partial \phi} .
\end{aligned}
$$

where $\overline{\mathbf{E}}$ and $\overline{\boldsymbol{S}}$ correspond to the intact material elasticity tensor and the intact Second Piola stress tensor, respectively . By intact we refer to the quantities that are derived directly from the hyperelastic potential, i.e. when no weighting factor is applied to account for damage.

In the following the derivatives on the right hand sides of equations (A.1)-(A.4) as well as $\mathrm{d} \boldsymbol{Y} / \mathrm{d} \nabla_{\boldsymbol{X}} \phi$ expression are derived. We specify these expressions consecutively according to their appearance in the above mentioned equations.

Recalling that $\Phi_{\mathrm{d}}(q, \kappa)=0$ for damage evolution, the tensorial derivative $\partial \kappa / \partial \boldsymbol{C}$ and 
$\partial \kappa / \partial \phi$ are deduced by means of the implicit function theorem as

$$
\begin{aligned}
2 \frac{\partial \kappa}{\partial \boldsymbol{C}} & =-2 \frac{\partial \Phi_{\mathrm{d}} / \partial \boldsymbol{C}}{\partial \Phi_{\mathrm{d}} / \partial \kappa}=-\vartheta_{\mathrm{d}} \overline{\boldsymbol{S}} \\
\frac{\partial \kappa}{\partial \phi} & =-\frac{\partial \Phi_{\mathrm{d}} / \partial \phi}{\partial \Phi_{\mathrm{d}} / \partial \kappa}=-\vartheta_{\mathrm{d}} \frac{\beta_{\mathrm{d}} \gamma_{\mathrm{d}}}{\eta_{\mathrm{d}} f_{\mathrm{d}}}
\end{aligned}
$$

where the abbreviation

$$
\vartheta_{\mathrm{d}}:=\left[\frac{\partial \Phi_{\mathrm{d}}}{\partial \kappa}\right]^{-1}=-1-\frac{\eta_{\mathrm{d}} f_{\mathrm{d}}}{\beta_{\mathrm{d}} \gamma_{\mathrm{d}}\left[\gamma_{\mathrm{d}}\left[1+\eta_{\mathrm{d}} \kappa\right]-\eta_{\mathrm{d}} \phi\right]}
$$

is introduced. Taking into account that $\partial_{\kappa} f_{\mathrm{d}}=-\eta_{\mathrm{d}} f_{\mathrm{d}}$ and $\partial_{\kappa} Y=-\beta_{\mathrm{d}} \gamma_{\mathrm{d}}$ as well as $\partial_{\phi} Y=\beta_{\mathrm{d}}$, we obtain the material tangent moduli as

$$
\begin{aligned}
\mathbf{E} & =f_{\mathrm{d}} \overline{\mathbf{E}}+\eta_{\mathrm{d}} f_{\mathrm{d}} \vartheta_{\mathrm{d}} \overline{\boldsymbol{S}} \otimes \overline{\boldsymbol{S}}, \\
\frac{\mathrm{d} \boldsymbol{S}}{\mathrm{d} \phi} & =\beta_{\mathrm{d}} \gamma_{\mathrm{d}} \vartheta_{\mathrm{d}} \overline{\boldsymbol{S}} \\
2 \frac{\mathrm{d} Y}{\mathrm{~d} \boldsymbol{C}} & =\beta_{\mathrm{d}} \gamma_{\mathrm{d}} \vartheta_{\mathrm{d}} \overline{\boldsymbol{S}} \\
\frac{\mathrm{d} Y}{\mathrm{~d} \phi} & =\beta_{\mathrm{d}}+\left[\beta_{\mathrm{d}} \gamma_{\mathrm{d}}\right]^{2}\left[\eta_{\mathrm{d}} f_{\mathrm{d}}\right]^{-1} \vartheta_{\mathrm{d}}, \\
\frac{\mathrm{d} Y}{\mathrm{~d} \nabla_{\boldsymbol{X}} \phi} & =c_{\mathrm{d}} \boldsymbol{C}^{-1} .
\end{aligned}
$$

For a purely elastic loading step, the tangent moduli can be reduced to $\mathbf{E}=f_{\mathrm{d}} \overline{\mathbf{E}}$ and $\mathrm{d} \boldsymbol{S} / \mathrm{d} \phi=2 \mathrm{~d} Y / \mathrm{d} \boldsymbol{C}=\mathbf{0}$ as well as $\mathrm{d} Y / \mathrm{d} \phi=\beta_{\mathrm{d}}$.

Applying push-forward operations to equations (A.8)-(A.12), we finally obtain the Cauchystress-related spatial tangent moduli as

$$
\begin{aligned}
\mathbf{e}=2 \frac{\mathrm{d} \boldsymbol{\sigma}}{\mathrm{d} \boldsymbol{g}} & =f_{\mathrm{d}} \overline{\mathbf{e}}+\eta_{\mathrm{d}} f_{\mathrm{d}} \vartheta_{\mathrm{d}} J \overline{\boldsymbol{\sigma}} \otimes \overline{\boldsymbol{\sigma}}, \\
\frac{\mathrm{d} \boldsymbol{\sigma}}{\mathrm{d} \phi} & =\beta_{\mathrm{d}} \gamma_{\mathrm{d}} \vartheta_{\mathrm{d}} \overline{\boldsymbol{\sigma}}, \\
2 \frac{\mathrm{d} y}{\mathrm{~d} \boldsymbol{g}} & =\beta_{\mathrm{d}} \gamma_{\mathrm{d}} \vartheta_{\mathrm{d}} \overline{\boldsymbol{\sigma}}, \\
\frac{\mathrm{d} y}{\mathrm{~d} \phi} & =J^{-1} \beta_{\mathrm{d}}\left[1+\beta_{\mathrm{d}} \gamma_{\mathrm{d}}^{2}\left[\eta_{\mathrm{d}} f_{\mathrm{d}}\right]^{-1} \vartheta_{\mathrm{d}}\right], \\
\frac{\mathrm{d} \boldsymbol{y}}{\mathrm{d} \nabla_{\boldsymbol{x}} \phi} & =J^{-1} c_{\mathrm{d}} \boldsymbol{I} .
\end{aligned}
$$

Note the relation $\partial_{\boldsymbol{g}}(\bullet):=\boldsymbol{F} \cdot \partial_{\boldsymbol{C}}(\bullet) \cdot \boldsymbol{F}^{\mathrm{t}}$; for detailed background on the formulation of anisotropic finite elasticity and inelasticity in terms of spatial arguments, the reader is referred to Menzel and Steinmann [67] and Menzel and Steinmann [136]. 



\section{BIBLIOGRAPHY}

[1] World Health Organization. Cardiovascular diseases (CVDs), January 2015. URL http://www. who.int/mediacentre/factsheets/fs317/en/.

[2] JE Lock, WR Castaneda-Zuniga, JL Bass, JE Foker, K Amplatz, and RW Anderson. Balloon dilatation of excised aortic coarctations. Radiology, 143(3):689-691, 1982.

[3] Robert H Beekman, Albert P Rocchini, MacDonald Dick, A Rebecca Snider, Dennis C Crowley, Gerald A Serwer, Robert L Spicer, and Amnon Rosenthal. Percutaneous balloon angioplasty for native coarctation of the aorta. Journal of the American College of Cardiology, 10(5):1078-1084, 1987.

[4] P Syamasundar Rao and Patrick Carey. Remodeling of the aorta after successful balloon coarctation angioplasty. Journal of the American College of Cardiology, 14 (5):1312-1317, 1989.

[5] P Syamasundar Rao. Coarctation of the aorta. Current cardiology reports, 7(6): 425-434, 2005.

[6] A.J. Schriefl, G. Zeindlinger, D.M. Pierce, P. Regitnig, and G.A. Holzapfel. Determination of the layer-specific distributed collagen fibre orientations in human thoracic and abdominal aortas and common iliac arteries. Journal of the Royal Society Interface, 9:1275-1286, 2012.

[7] T.C. Gasser, R.W. Ogden, and G.A. Holzapfel. Hyperelastic modelling of arterial layers with distributed collagen fibre orientations. Journal of the Royal Society Interface, 3:15-35, 2006.

[8] Mary E Klingensmith et al. The Washington manual of surgery. Lippincott Williams \& Wilkins, 2008.

[9] JB Duguid and WB Robertson. Mechanical factors in atherosclerosis. The Lancet, 269(6981):1205-1209, 1957.

[10] Karl Schmid, William O McSharry, Cornelis H Pameijer, and J Paul Binette. Chemical and physicochemical studies on the mineral deposits of the human atherosclerotic aorta. Atherosclerosis, 37(2):199-210, 1980.

[11] Lewis Wexler, Bruce Brundage, John Crouse, Robert Detrano, Valentin Fuster, Jamshid Maddahi, John Rumberger, William Stanford, Richard White, Kathryn 
Taubert, et al. Coronary artery calcification: Pathophysiology, epidemiology, imaging methods, and clinical implications a statement for health professionals from the american heart association. Circulation, 94(5):1175-1192, 1996.

[12] Y.C. Fung. Biomechanics: mechanical properties of living tissues. Springer, 1993.

[13] Jay D Humphrey. Cardiovascular solid mechanics: cells, tissues, and organs. Springer Science \& Business Media, 2002.

[14] P Martins, E Pena, RM Natal Jorge, A Santos, L Santos, T Mascarenhas, and B Calvo. Mechanical characterization and constitutive modelling of the damage process in rectus sheath. Journal of the mechanical behavior of biomedical materials, $8: 111-122,2012$.

[15] R.F. Fuchs. Zurphysiologie undwachstumsmechanik des blutgefa systems. Arch. Ges. Physiol., 28, 1900.

[16] CJ Chuong and YC Fung. Residual stress in arteries. In Frontiers in Biomechanics, pages 117-129. Springer, 1986.

[17] Markus J Buehler. Nature designs tough collagen: explaining the nanostructure of collagen fibrils. Proceedings of the National Academy of Sciences, 103(33):1228512290, 2006.

[18] Markus J Buehler. Nanomechanics of collagen fibrils under varying cross-link densities: atomistic and continuum studies. Journal of the Mechanical Behavior of Biomedical Materials, 1(1):59-67, 2008.

[19] Matthew D Shoulders and Ronald T Raines. Collagen structure and stability. Annual review of biochemistry, 78:929, 2009.

[20] Alfonso Gautieri, Simone Vesentini, Alberto Redaelli, and Markus J Buehler. Hierarchical structure and nanomechanics of collagen microfibrils from the atomistic scale up. Nano letters, 11(2):757-766, 2011.

[21] Zhilei Liu Shen, Harold Kahn, Roberto Ballarini, and Steven J Eppell. Viscoelastic properties of isolated collagen fibrils. Biophysical journal, 100(12):3008-3015, 2011.

[22] José F Rodríguez, Fernando Cacho, José A Bea, and Manuel Doblaré. A stochasticstructurally based three dimensional finite-strain damage model for fibrous soft tissue. Journal of the Mechanics and Physics of Solids, 54(4):864-886, 2006.

[23] Markus J Buehler. Atomistic and continuum modeling of mechanical properties of collagen: elasticity, fracture, and self-assembly. Journal of Materials Research, 21 (08):1947-1961, 2006.

[24] Y. Tang, R. Ballarini, M.J. Buehler, and S.J. Eppell. Deformation micromechanisms of collagen fibrils under uniaxial tension. Journal of The Royal Society Interface, 7 (46):839-850, 2010.

[25] Christian T Gasser. An irreversible constitutive model for fibrous soft biological tissue: a 3-d microfiber approach with demonstrative application to abdominal aortic aneurysms. Acta Biomaterialia, 7(6):2457-2466, 2011. 
[26] Giampaolo Martufi and T Christian Gasser. A constitutive model for vascular tissue that integrates fibril, fiber and continuum levels with application to the isotropic and passive properties of the infrarenal aorta. Journal of biomechanics, 44(14):2544-2550, 2011.

[27] Giampaolo Martufi and T Christian Gasser. Turnover of fibrillar collagen in soft biological tissue with application to the expansion of abdominal aortic aneurysms. Journal of The Royal Society Interface, 9(77):3366-3377, 2012.

[28] L.M. Kachanov. Time of the rupture process under creep conditions. Izvestija Akademii Nauk Sojuza Sovetskich Socialisticeskich Respubliki (SSSR) Otdelenie Techniceskich Nauk (Moskra), 8:26-31, 1958.

[29] L.M. Kachanov. Introduction to Continuum Damage Mechanics. Martinus Nijhoff Publishers, Dordrecht, The Netherlands., 1986.

[30] D. Krajcinovic and J. Lemaitre. Continuum Damage Mechanics. Number 295 in CISM Courses and Lectures. Springer, 1987.

[31] J. Lemaitre and J.-L. Chaboche. Mechanics of Solid Materials. Cambridge University Press, Cambridge, 1990.

[32] J. Lemaitre. A Course on Damage Mechanics. Springer, 2nd edition, 1996.

[33] D. Krajcinovic. Damage Mechanics. North-Holland, Amsterdam, 1996.

[34] A.C. Eringen. Nonlocal Continuum Field Theories. Springer, 2002.

[35] E.C. Aifantis. On the role of gradients in the localization of deformation and fracture. International Journal of Engineering Science, 30(10):1279-1299, 1992.

[36] E.C. Aifantis. On the gradient approach - Relation to Eringen's nonlocal theory. International Journal of Engineering Science, 49(12):1367-1377, 2011.

[37] A.C. Eringen, editor. Continuum Physics. Volume IV - Polar and Nonlocal Field Theories. Academic Press, 1976.

[38] D. Rogula, editor. Nonlocal Theory of Material Media. Number 268 in CISM Courses and Lectures. Springer, 1982.

[39] G. Pijaudier-Cabot and Z.P. Bažant. Nonlocal damage theory. Journal of Engineering Mechanics, 113(10):1512-1533, 1987.

[40] Z.P. Bažant. Why continuum damage is nonlocal: Micromechanics arguments. Journal of Engineering Mechanics, 117(5):1070-1087, 1991.

[41] M. Jirásek. Nonlocal models for damage and fracture: Comparison of approaches. International Journal of Solids and Structures, 35(31-32):4133-4145, 1998.

[42] Z.P. Bažant and J. Ožbolt. Nonlocal microplane model for fracture, damage, and size effect in structures. Journal of Engineering Mechanics, 116(11):2485-2505, 1990. 
[43] Z.P. Bažant and G. Di Luzio. Nonlocal microplane model with strain-softening yield limits. International Journal of Solids and Structures, 41(24-25):7209-7240, 2004.

[44] D. Lasry and T. Belytschko. Localization limiters in transient problems. International Journal of Solids and Structures, 24(6):581-597, 1988.

[45] H.-B. Mühlhaus and E.C. Alfantis. A variational principle for gradient plasticity. International Journal of Solids and Structures, 28(7):845-857, 1991.

[46] C. Polizzotto, G. Borino, and P. Fuschi. A thermodynamically consistent formulation of nonlocal and gradient plasticity. Mechanics Research Communications, 25(1):7582, 1998 .

[47] J.H.P. de Vree, W.A.M. Brekelmans, and M.A.J. van Gils. Comparison of nonlocal approaches in continuum damage mechanics. Computers \& Structures, 55(4):581-588, 1995.

[48] R. H. J. Peerlings, R. de Borst, W. A. M. Brekelmans, and J. H. P. de Vree. Gradient enhanced damage for quasi-brittle materials. International Journal for Numerical Methods in Engineering, 39(19):3391-3403, 1996.

[49] R. de Borst and J. Pamin. Some novel developments in finite element procedures for gradient-dependent plasticity. International Journal for Numerical Methods in Engineering, 39(14):2477-2505, 1996.

[50] J. Pamin. Gradient plasticity and damage models: a short comparison. Computational Materials Science, 32(3-4):472-479, 2005.

[51] T. Svedberg and K. Runesson. A thermodynamically consistent theory of gradientregularized plasticity coupled to damage. International Journal of Plasticity, 13: 669-696, 1997.

[52] E. Kuhl and E. Ramm. Simulation of strain localization with gradient enhanced damage models. Computational Materials Science, 16(1-4):176-185, 1999.

[53] E. Kuhl, E. Ramm, and R. de Borst. An anisotropic gradient damage model for quasi-brittle materials. Computer Methods in Applied Mechanics and Engineering, 183(1-2):87-103, 2000.

[54] T. Liebe, P. Steinmann, and A. Benallal. Theoretical and computational aspects of a thermodynamically constistent framework for geometrically linear gradient damage. Computer Methods in Applied Mechanics and Engineering, 190:6555-6576, 2001.

[55] B. J. Dimitrijević and K. Hackl. A method for gradient enhancement of continuum damage models. Technische Mechanik, 28(1):43-52, 2008.

[56] P. Steinmann. Formulation and computation of geometrically non-linear gradient damage. International Journal for Numerical Methods in Engineering, 46:757-779, 1999 .

[57] T. Liebe and P. Steinmann. Two strategies towards geometrically non-linear isotropic gradient damage. Journal of the Mechanical Behavior of Materials, 13:175-194, 2002. 
[58] T. Liebe, A. Menzel, and P. Steinmann. Theory and numerics of a thermodynamically consistent framework for geometrically non-linear gradient plasticity. Int. J. Engng. Sci., 41:1603-1629, 2003.

[59] M. Frémond and B. Nedjar. Damage, gradient of damage and principle of virtual power. International Journal of Solids and Structures, 33(8):1083-1103, 1996.

[60] B. Nedjar. A theoretical and computational setting for a geometrically nonlinear gradient damage modelling framework. Computational Mechanics, 30(1):65-80, 2002.

[61] R.K. Abu Al-Rub and G.Z. Voyiadjis. A finite strain plastic-damage model for high velocity impact using combined viscosity and gradient localization limiters: Part I theoretical formulation. International Journal of Damage Mechanics, 15(4):293-334, 2006.

[62] G.Z. Voyiadjis and R.K. Abu Al-Rub. A finite strain plastic-damage model for high velocity impact using combined viscosity and gradient localization limiters: Part II - Numerical Aspects and Simulations. International Journal of Damage Mechanics, 15(4):335-373, 2006.

[63] PJ Flory. Thermodynamic relations for high elastic materials. Trans. Faraday Soc., 57:829-838, 1961.

[64] R.W. Ogden. Nearly isochoric elastic deformations: Application to rubberlike solids. J. Mech. Phys. Solids, (26):37-57, 1978.

[65] A.J.M. Spencer. Deformations of Fibre-reinforced Materials. Oxford University Press, 1972.

[66] J.A. Weiss, B.N. Maker, and S. Govindjee. Finite element implementation of incompressible, transversely isotropic hyperelasticity. Computer Methods in Applied Mechanics and Engineering, 135:107-128, 1996.

[67] A. Menzel and P. Steinmann. A view on anisotropic finite hyper-elasticity. European Journal of Mechanics - A/Solids, 22:71-87, 2003.

[68] A. Menzel, M. Harrysson, and M. Ristinmaa. Towards an orientation-distributionbased multi-scale approach for remodelling biological tissues. Computer Methods in Biomechanics and Biomedical Engineering, 11(5):505-524, 2008.

[69] B.D. Coleman and W. Noll. The thermodynamics of elastic materials with heat conduction and viscosity. Archive for Rational Mechanics and Analysis, 13(1):167$178,1963$.

[70] Bernard David Coleman and Morton E Gurtin. Thermodynamics with internal state variables. 1967.

[71] J.C. Simo. On a fully three-dimensional finite-strain viscoelastic damage model: Formulation and computational aspects. Computer Methods in Applied Mechanics and Engineering, 60:153-173, 1987. 
[72] G.A. Holzapfel, T.C. Gasser, and R.W. Ogden. A new constitutive framework for arterial wall mechanics and a comparative study of material models. Journal of Elasticity, 61:1-48, 2000.

[73] G.A. Holzapfel and R.W. Ogden. Constitutive modelling of arteries. Proceedings of the Royal Society A. Mathematical, Physical and Engineering Sciences, 466:15511597, 2010.

[74] A. Menzel, M. Harrysson, and M. Ristinmaa. Towards an orientation-distributionbased multi-scale approach for remodelling biological tissues. Computer Methods in Biomechanics and Biomedical Engineering, 11(5):505-524, 2008.

[75] Eric Baer, James J Cassidy, and Anne Hiltner. Hierarchical structure of collagen composite systems. Pure Appl. Chem, 63:961-973, 1991.

[76] Naoki Sasaki and Shingo Odajima. Elongation mechanism of collagen fibrils and force-strain relations of tendon at each level of structural hierarchy. Journal of biomechanics, 29(9):1131-1136, 1996.

[77] Estefanı Peña, Manuel Doblaré, et al. An anisotropic pseudo-elastic approach for modelling mullins effect in fibrous biological materials. Mechanics Research Communications, 36(7):784-790, 2009.

[78] JC Simo. On a fully three-dimensional finite-strain viscoelastic damage model: formulation and computational aspects. Computer methods in applied mechanics and engineering, 60(2):153-173, 1987.

[79] JC Simo and JW Ju. Strain-and stress-based continuum damage models-I. Formulation. International Journal of Solids and Structures, 23(7):821-840, 1987.

[80] GN Wells and LJ Sluys. Application of embedded discontinuities for softening solids. Engineering Fracture Mechanics, 65(2):263-281, 2000.

[81] B.H. Oh and Z.P. Bazant. Crack band theory for fracture of concrete. Materials and Structures, January-February, pages 155-177, 1983.

[82] J.C. Simo, J. Oliver, and F. Armero. An analysis of strong discontinuities induced by strain-softening in rate-independent inelastic solids. Computational Mechanics, 12 (5):277-296, 1993.

[83] J. Oliver. A consistent characteristic length for smeared cracking models. International Journal for Numerical Methods in Engineering, 28(2):461-474, 1989.

[84] J.A. Petruska and A.J. Hodge. A subunit model for the tropocollagen macromolecule. Proceedings of the National Academy of Sciences of the United States of America, 51 (5):871, 1964.

[85] Marc André Meyers, Po-Yu Chen, Albert Yu-Min Lin, and Yasuaki Seki. Biological materials: structure and mechanical properties. Progress in Materials Science, 53(1): 1-206, 2008. 
[86] V Ottani, M Raspanti, and A Ruggeri. Collagen structure and functional implications. Micron, 32(3):251-260, 2001.

[87] A.J. Bailey and T.J. Sims. Chemistry of the collagen cross-links. nature of the crosslinks in the polymorphic forms of dermal collagen during development. Biochem. $J$, 153:211-215, 1976.

[88] Arnab Bhattacharjee and Manju Bansal. Collagen structure: the madras triple helix and the current scenario. Iubmb Life, 57(3):161-172, 2005.

[89] Jun Liao, Lin Yang, Jonathan Grashow, and Michael S Sacks. Molecular orientation of collagen in intact planar connective tissues under biaxial stretch. Acta biomaterialia, 1(1):45-54, 2005.

[90] Alicia Claudia Lorenzo and Ernesto Raúl Caffarena. Elastic properties, young's modulus determination and structural stability of the tropocollagen molecule: a computational study by steered molecular dynamics. Journal of biomechanics, 38(7): 1527-1533, 2005.

[91] Tatsuo Ushiki. Collagen fibers, reticular fibers and elastic fibers. a comprehensive understanding from a morphological viewpoint. Archives of histology and cytology, 65(2):109-126, 2002.

[92] NJB Driessen, GWM Peters, JM Huyghe, CVC Bouten, and FPT Baaijens. Remodelling of continuously distributed collagen fibres in soft connective tissues. Journal of biomechanics, 36(8):1151-1158, 2003.

[93] Gerhard Holzapfel, Thomas Gasser, and Ray Ogden. A new constitutive framework for arterial wall mechanics and a comparative study of material models. Journal of Elasticity, 61:1-48, 2000. 10.1023/A:1010835316564.

[94] Eric Jones, Travis Oliphant, and Pearu Peterson. Scipy: Open source scientific tools for python. 2001.

[95] Zhilei L Shen, Mohammad Reza Dodge, Harold Kahn, Roberto Ballarini, and Steven J Eppell. Stress-strain experiments on individual collagen fibrils. Biophysical Journal, 95(8):3956-3963, 2008.

[96] Zhilei Liu Shen, Mohammad Reza Dodge, Harold Kahn, Roberto Ballarini, and Steven J Eppell. In vitro fracture testing of submicron diameter collagen fibril specimens. Biophysical journal, 99(6):1986-1995, 2010.

[97] Gerhard Sommer and Gerhard A Holzapfel. 3d constitutive modeling of the biaxial mechanical response of intact and layer-dissected human carotid arteries. Journal of the mechanical behavior of biomedical materials, 5(1):116-128, 2012.

[98] Hannah Weisbecker, David M Pierce, Peter Regitnig, and Gerhard A Holzapfel. Layer-specific damage experiments and modeling of human thoracic and abdominal aortas with non-atherosclerotic intimal thickening. Journal of the mechanical behavior of biomedical materials, 12:93-106, 2012. 
[99] David M Pierce, Franz Maier, Hannah Weisbecker, Christian Viertler, Peter Verbrugghe, Nele Famaey, Inge Fourneau, Paul Herijgers, and Gerhard A Holzapfel. Human thoracic and abdominal aortic aneurysmal tissues: damage experiments, statistical analysis and constitutive modeling. Journal of the Mechanical Behavior of Biomedical Materials, 2014.

[100] T. Christian Gasser, Ray W Ogden, and Gerhard A Holzapfel. Hyperelastic modelling of arterial layers with distributed collagen fibre orientations. Journal of The Royal Society Interface, 3(6):15-35, 2006.

[101] G. Sommer, T.C. Gasser, P. Regitnig, M. Auer, and G.A. Holzapfel. Dissection properties of the human aortic media: an experimental study. Journal of biomechanical engineering, 130:021007, 2008.

[102] D.A. Vorp, ML Raghavan, S.C. Muluk, M.S. Makaroun, D.L. Steed, R. Shapiro, and M.W. Webster. Wall strength and stiffness of aneurysmal and nonaneurysmal abdominal aorta. Annals of the New York Academy of Sciences, 800(1):274-276, 1996.

[103] Mustapha Adham, Jean-Paul Gournier, Jean-Pierre Favre, Eric De La Roche, Christian Ducerf, Jacques Baulieux, Xavier Barral, and Michel Pouyet. Mechanical characteristics of fresh and frozen human descending thoracic aorta. Journal of Surgical Research, 64(1):32-34, 1996.

[104] IM Tiessen and MR Roach. Factors in the initiation and propagation of aortic dissections in human autopsy aortas. Journal of biomechanical engineering, 115(1): 123-125, 1993.

[105] Michael W Carson and Margot R Roach. The strength of the aortic media and its role in the propagation of aortic dissection. Journal of biomechanics, 23(6):579-588, 1990 .

[106] Robert L Taylor. Feap-a finite element analysis program. Version 7.5 Theory Manual, 2003.

[107] Eric Jones, Travis Oliphant, Pearu Peterson, et al. SciPy: Open source scientific tools for Python, 2001.

[108] R. H. J. Peerlings, R. de Borst, W. A. M. Brekelmans, and M. G. D. Geers. Gradientenhanced damage modelling of concrete fracture. Mechanics of Cohesive-frictional Materials, 3(4):323-342, 1998.

[109] A. Simone, H. Askes, R.H.J. Peerlings, and L.J. Sluys. Interpolation requirements for implicit gradient-enhanced continuum damage models. Communications in $\mathrm{Nu}$ merical Methods in Engineering, 19(7):563-572, 2003.

[110] Tobias Waffenschmidt, César Polindara, Andreas Menzel, and Sergio Blanco. A gradient-enhanced large-deformation continuum damage model for fibre-reinforced materials. Computer Methods in Applied Mechanics and Engineering, 268(0):801 $842,2014$. 
[111] J.C. Simo and T.J.R. Hughes. Computational Inelasticity, volume 7 of Interdisciplinary Applied Mathematics. Springer, 1998.

[112] T.J.R. Hughes. The Finite Element Method. Dover, 2000.

[113] JC Simo, RL Taylor, and KS Pister. Variational and projection methods for the volume constraint in finite deformation elasto-plasticity. Computer Methods in Applied Mechanics and Engineering, 51(1):177-208, 1985.

[114] P. Wriggers. Nonlinear Finite Element Methods. Springer, 2008.

[115] Ch Miehe. Aspects of the formulation and finite element implementation of large strain isotropic elasticity. International Journal for Numerical Methods in Engineering, 37(12):1981-2004, 1994.

[116] Abaqus 6.11 Documentation. Dassault Systèmes Simulia Corp., 2011.

[117] René de Borst. Damage, Material Instabilities, and Failure. John Wiley \& Sons, Ltd, 2004. ISBN 9780470091357.

[118] T. Waffenschmidt and A. Menzel. Extremal states of energy of a double-layered thickwalled tube - application to residually stressed arteries. Journal of the Mechanical Behavior of Biomedical Materials, available online, (doi:10.1016/j.jmbbm.2013.05.023), 2013.

[119] Gerhard A Holzapfel, Gerhard Sommer, and Peter Regitnig. Anisotropic mechanical properties of tissue components in human atherosclerotic plaques. Journal of biomechanical engineering, 126(5):657-665, 2004.

[120] G.A. Holzapfel, M. Stadler, and C.A.J. Schulze-Bauer. A layer-specific threedimensional model for the simulation of balloon angioplasty using magnetic resonance imaging and mechanical testing. Annals of Biomedical Engineering, 30:753-767, 2002.

[121] GerhardA. Holzapfel, Michael Stadler, and ChristianA.J. Schulze-Bauer. A layerspecific three-dimensional model for the simulation of balloon angioplasty using magnetic resonance imaging and mechanical testing. Annals of Biomedical Engineering, 30(6):753-767, 2002.

[122] Christian Miehe. Numerical computation of algorithmic (consistent) tangent moduli in large-strain computational inelasticity. Computer Methods in Applied Mechanics and Engineering, 134(3i4):223 - 240, 1996.

[123] T.C. Gasser and G.A. Holzapfel. Finite element modeling of balloon angioplasty by considering overstretch of remnant non-diseased tissues in lesions. Computational Mechanics, 40:47-60, 2007.

[124] DimitriosE. Kiousis, AlexanderR. Wulff, and GerhardA. Holzapfel. Experimental studies and numerical analysis of the inflation and interaction of vascular balloon catheter-stent systems. Annals of Biomedical Engineering, 37(2):315-330, 2009.

[125] V. Alastrué, J.F. Rodríguez, B. Calvo, and M. Doblaré. Structural damage models for fibrous biological soft tissues. International Journal of Solids and Structures, 44 (18-19):5894-5911, 2007. 
[126] V Alastrué. Some Inelastic Problems in the Modelling of Blood Vessels: Applications to Non-physiological States and Vascular Surgery. Víctor Alastrué, 2008.

[127] B. Calvo, E. Peña, M.A. Martínez, and M. Doblaré. An uncoupled directional damage model for fibred biological soft tissues. Formulation and computational aspects. International Journal for Numerical Methods in Engineering, 69(10):2036-2057, 2007.

[128] K.Y. Volokh. Hyperelasticity with softening for modeling materials failure. Journal of the Mechanics and Physics of Solids, 55(10):2237-2264, 2007.

[129] D. Balzani, S. Brinkhues, and G.A. Holzapfel. Constitutive framework for the modeling of damage in collagenous soft tissues with application to arterial walls. Computer Methods in Applied Mechanics and Engineering, 213-216(0):139-151, 2012.

[130] Ingo Müller and Henning Struchtrup. Inflating a rubber balloon. Mathematics and Mechanics of Solids, 7(5):569-577, 2002.

[131] Ingo Müller and Peter Strehlow. Rubber and rubber balloons: paradigms of thermodynamics. Springer Science \& Business Media, 2004.

[132] G.A. Holzapfel and R.W. Ogden, editors. Biomechanics of Soft Tissue in Cardiovascular Systems. Number 441 in CISM Courses and Lectures. Springer, 2003.

[133] V. Alastrué, M.A. Martínez, M. Doblaré, and A. Menzel. Anisotropic micro-spherebased finite elasticity applied to blood vessel modelling. Journal of the Mechanics and Physics of Solids, 57:178-203, 2009.

[134] Andreas Menzel Tobias Waffenschmidt, César Polindara. A gradient-enhanced continuum damage model for residually stressed fibre-reinforced materials at finite strains. In Biomedical Technology, pages 19-40. Springer, 2015.

[135] Gerhard A Holzapfel. Nonlinear Solid Mechanics. Wiley Chichester, 2000.

[136] A. Menzel and P. Steinmann. On the spatial formulation of anisotropic multiplicative elasto-plasticity. Computer Methods in Applied Mechanics and Engineering, 192: 3431-3470, 2003. 

